\title{
Orchestrating Communications in a Three-Type Chirality Totem: Remote Control of the Chiroptical Response of a Möbius Aromatic System
}

Rajaa Benchouaia, Nicolas Cissé, Bernard Boitrel, Matthieu Sollogoub, Stéphane Le Gac* and Mickaël Ménand*

\section{Supporting Information}




\section{Content}

1 General information

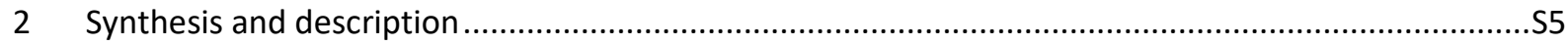

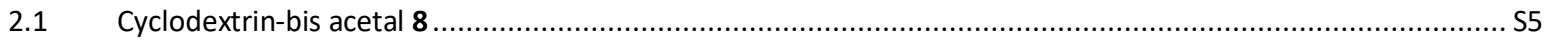

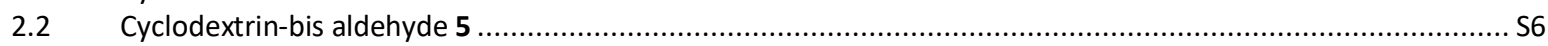

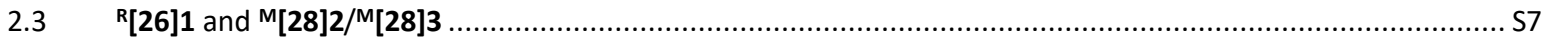

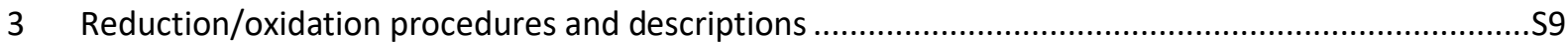

3.1 General scheme of the red/ox interconversion of all HCD hybrids .......................................................... S9

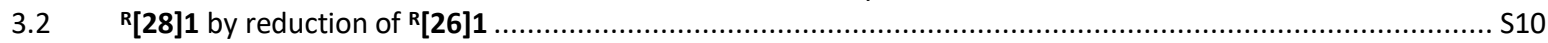

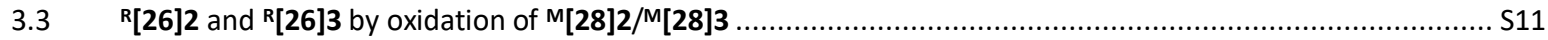

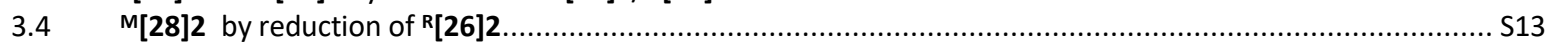

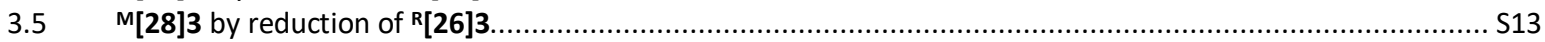

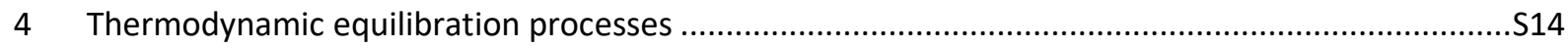

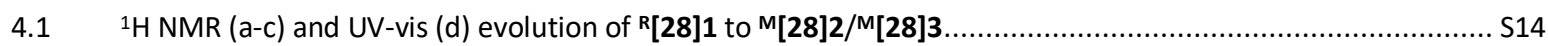

$4.2{ }^{1} \mathrm{H}$ and ${ }^{19} \mathrm{~F}$ NMR evolution of ${ }^{\mathrm{M}}[\mathbf{2 8}] 2$ (a) and ${ }^{\mathrm{M}}[\mathbf{2 8}] \mathbf{3}$ (b) to ${ }^{\mathrm{M}}[\mathbf{2 8}] 2 /{ }^{\mathrm{M}}[\mathbf{2 8}] 3$ (spectra recorded in $\mathrm{CDCl}_{3}$ at $298 \mathrm{~K}$ ) $\mathrm{S} 15$

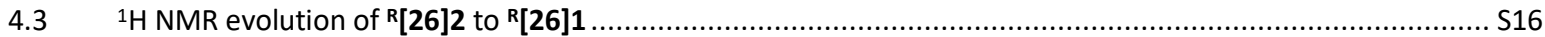

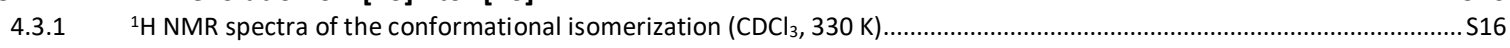

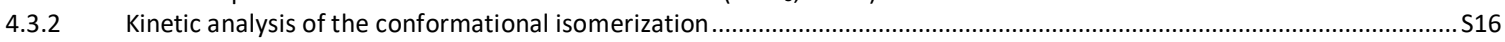

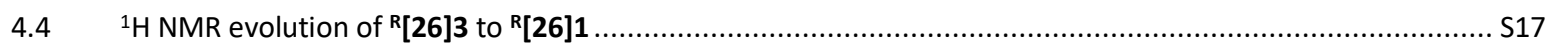

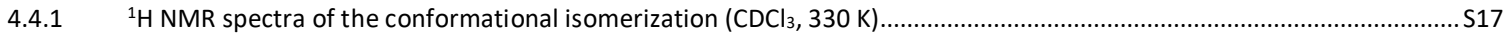

4.4.2 Kinetic analysis of the conformational isomerization ..........................................................................................

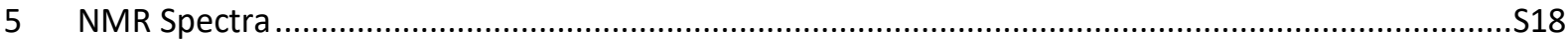

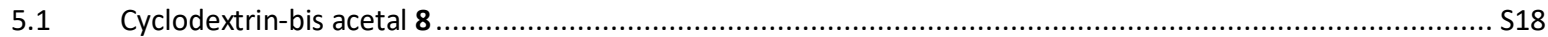

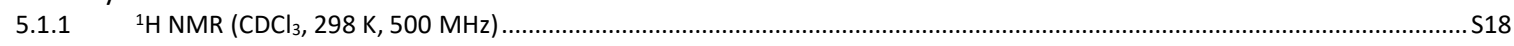

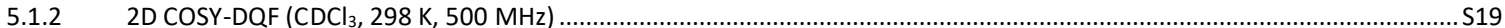

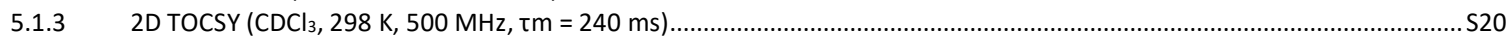

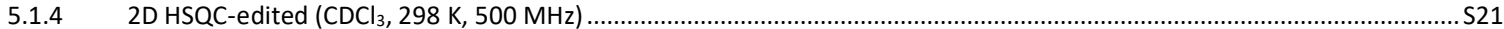

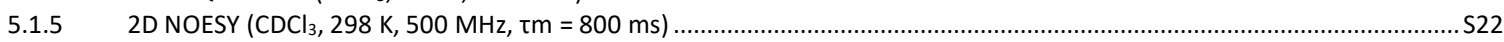

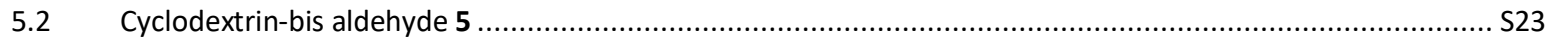

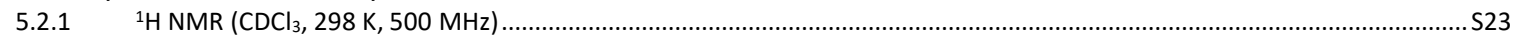

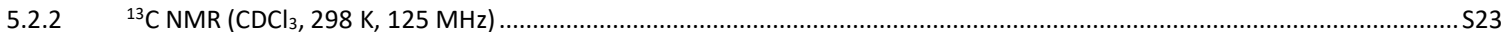

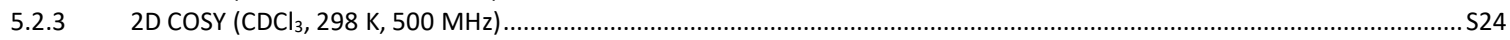

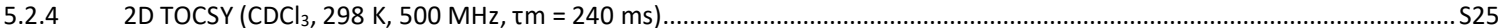

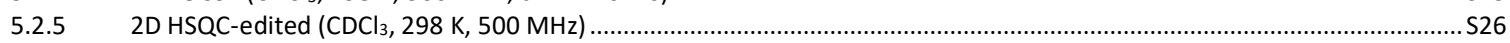

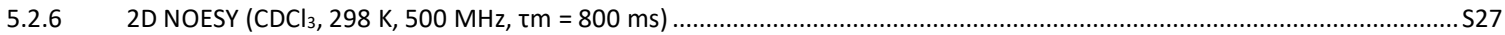

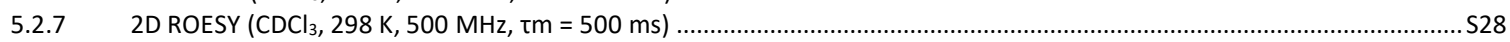

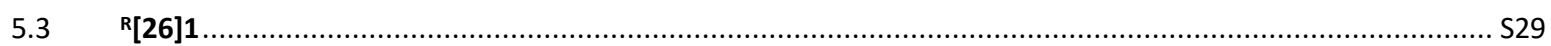

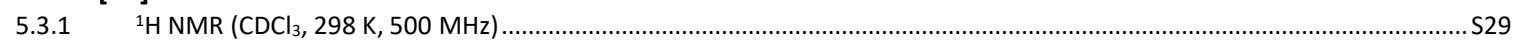

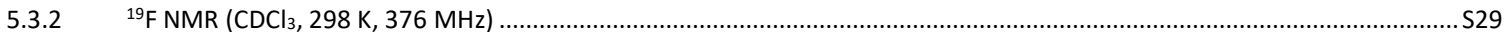

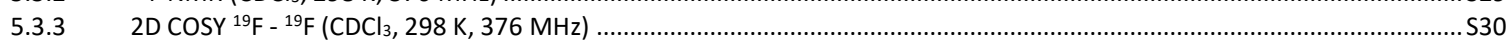

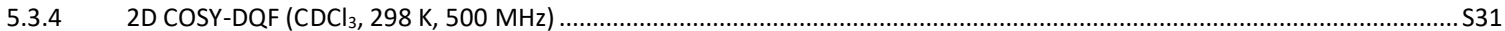

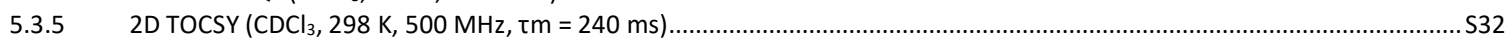

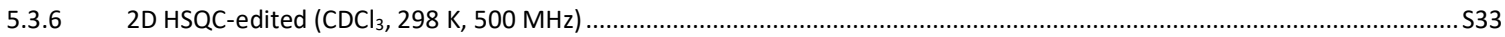

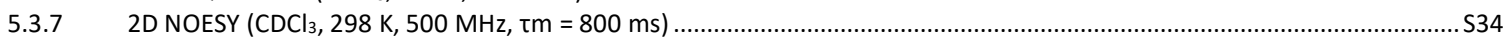

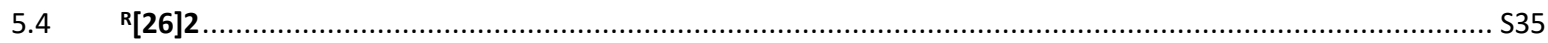

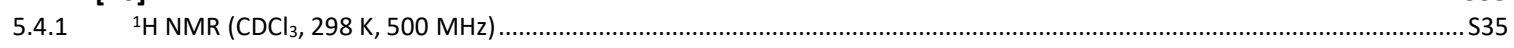

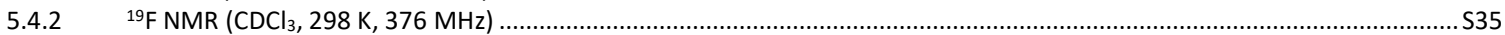

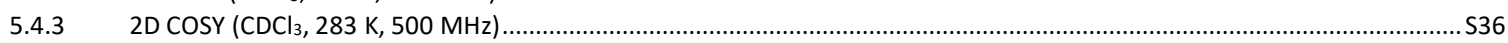

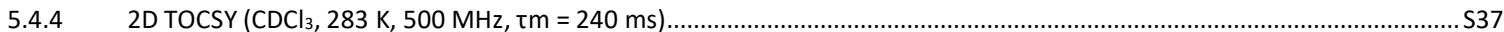

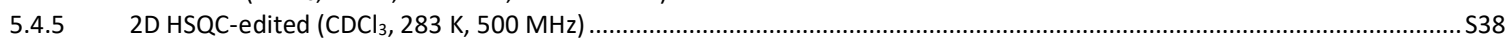

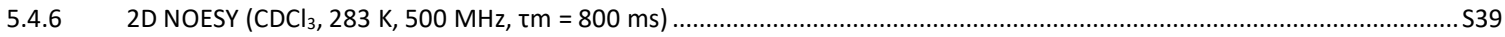

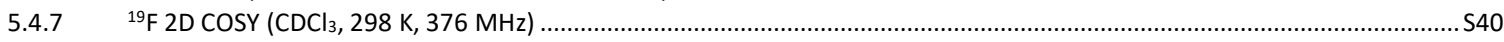

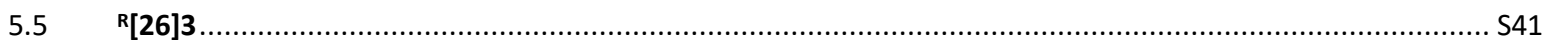




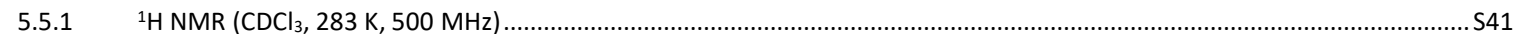

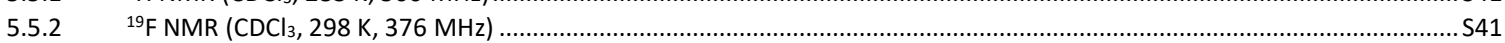

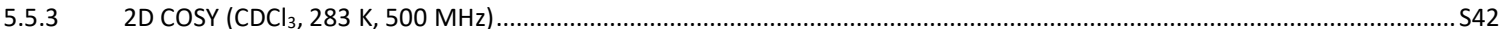

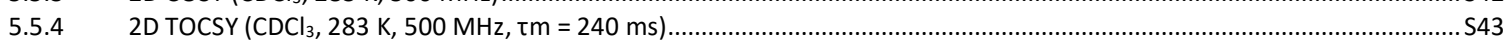

5.5.5 2D HSQC-edited ( $\left.\mathrm{CDCl}_{3}, 283 \mathrm{~K}, 500 \mathrm{MHz}\right) \ldots \ldots \ldots \ldots \ldots . . . .544$

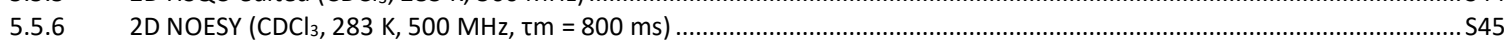

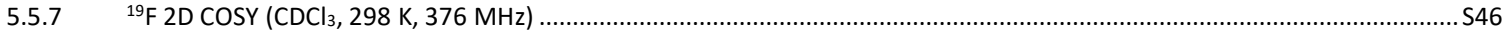

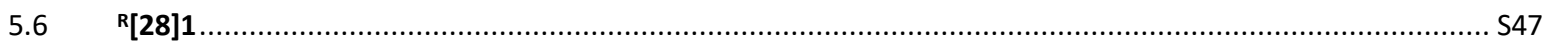

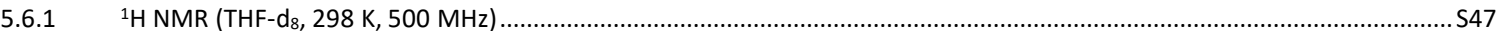

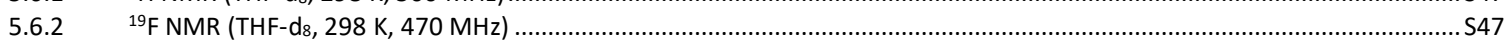

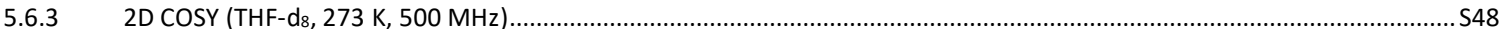

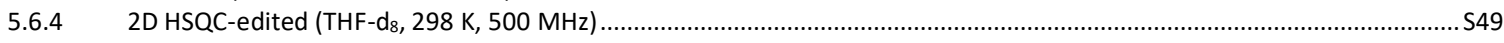

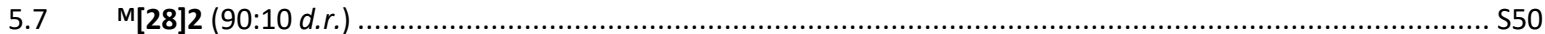

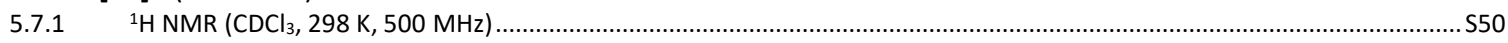

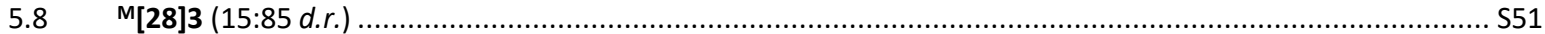

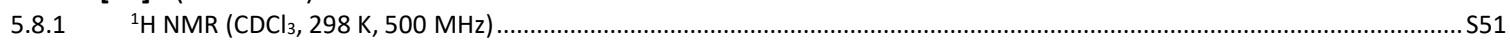

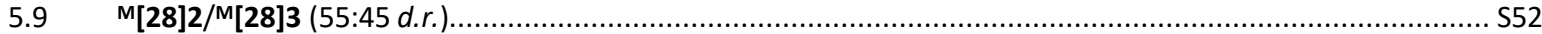

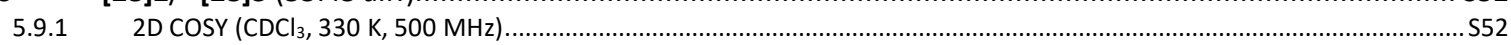

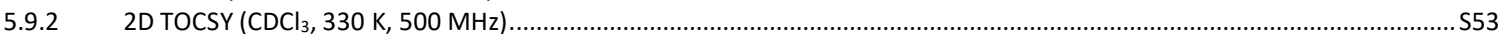

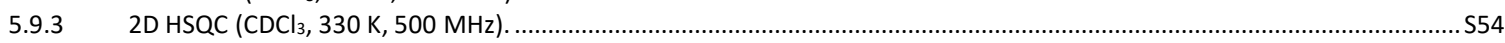

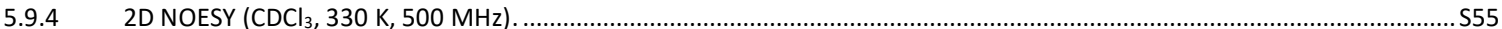

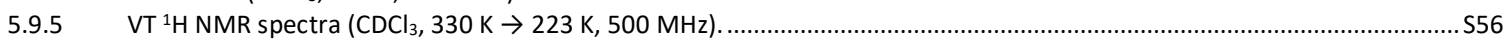

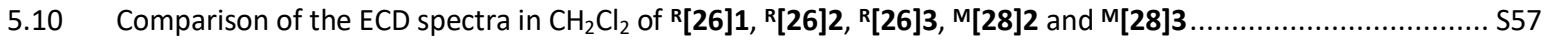

6 Geometric comparison of the formal Möbius diastereomers of ${ }^{\mathrm{M}}[\mathbf{2 8}] 2$ and ${ }^{\mathrm{M}}[28] 3$

6.1 Reassignment of the absolute configuration of the [28]hexaphyrin palladium complex $2 \mathrm{~A}$ (X-ray structure taken from Osuka et al. Angew. Chem. Int. Ed. 2010, 49, 6619; CCDC 767696), exhibiting negative Cotton effect. .... S58

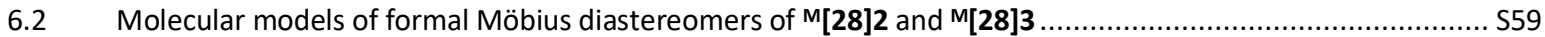

7 Metalation .560

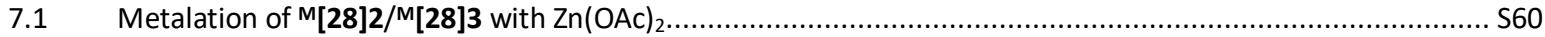

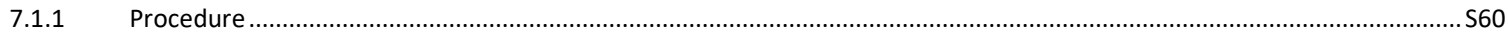

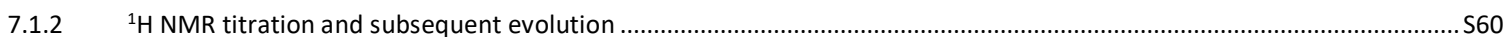

7.1.3 VT ${ }^{1}{ }^{H}$ NMR of (M)-M[28]2·ZnL ${ }^{1} /(\mathrm{P})-{ }^{\mathrm{M}}[\mathbf{2 8}] 3 \cdot \mathrm{ZnL}^{1}$ and $2 \mathrm{D}$ HSQC and NOESY NMR spectra $\left(\mathrm{CDCl}_{3} / \mathrm{CD}_{3} \mathrm{OD}, 9: 1,260 \mathrm{~K}\right.$ selected

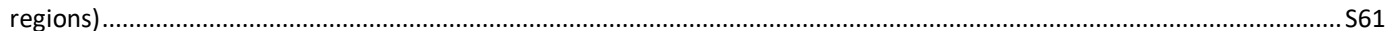

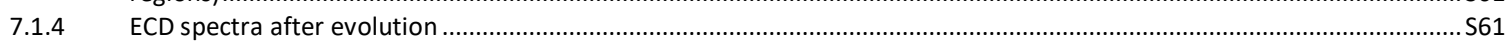

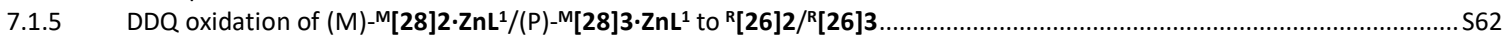

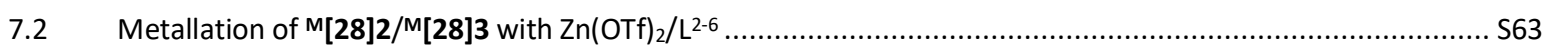

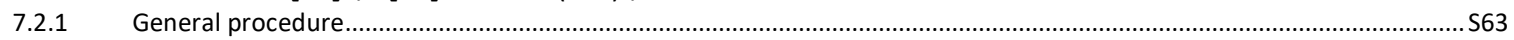

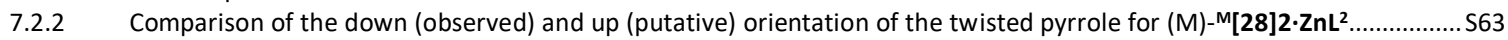

7.2.3 Distances between protons involved in NOE correlations with the bound L ${ }^{2}$ of (M)-M $[28] 2 \cdot \mathbf{Z n L}^{2}$ (exo-complexation) and

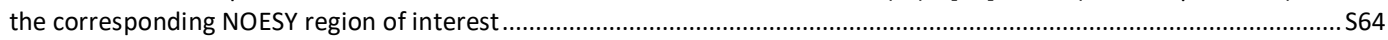

7.2.4 ${ }^{1} \mathrm{H} \mathrm{NMR}\left(\mathrm{CDCl}_{3} / \mathrm{CD}_{3} \mathrm{OD} 9: 1,500 \mathrm{MHz}, 298 \mathrm{~K}\right)$ titration with $\mathrm{Zn}(\mathrm{OTf})_{2} / \mathrm{L}^{2}$ and subsequent evolution...................................... 665

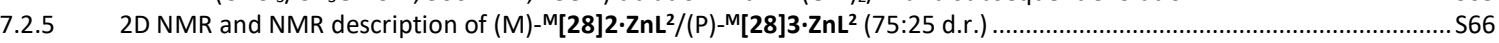

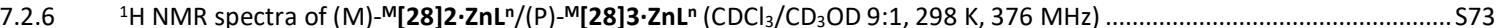

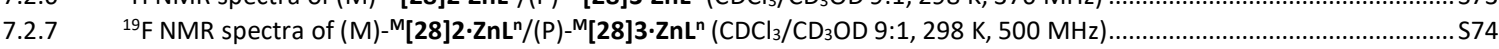

7.2.8 Normalized UV-vis absorption spectra of (M)- ${ }^{\mathrm{M}}[\mathbf{2 8}] \mathbf{2} \cdot \mathbf{Z n L}{ }^{\mathrm{n}} /(\mathrm{P})-{ }^{\mathrm{M}}[\mathbf{2 8}] \mathbf{3} \cdot \mathrm{ZnL}^{\mathrm{n}}\left(\mathrm{CHCl}_{3} / \mathrm{CH}_{3} \mathrm{OH}\right.$ 9:1) ...................................... S75

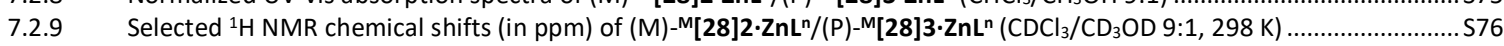




\section{General information}

Commercial products were used as received. Anhydrous THF and $\mathrm{CH}_{2} \mathrm{Cl}_{2}$ were obtained by distillation over $\mathrm{Na}$ /benzophenone and $\mathrm{CaH}_{2}$, respectively. Pyrrole was filtered over basic alumina and degassed by argon bubbling before used. ${ }^{1} \mathrm{H}$ NMR spectra were recorded at $300 \mathrm{~K}$ (unless otherwise stated) at $500 \mathrm{MHz}$ or $600 \mathrm{MHz} .{ }^{13} \mathrm{C}$ NMR spectra were recorded at $300 \mathrm{~K}$ (unless otherwise stated) at $150 \mathrm{MHz}$. Residual traces of solvent were used as internal standard. Chemical shifts are expressed in ppm (abbreviations for multiplicities and descriptors are $s=$ singlet [ $\mathrm{s}_{\mathrm{b}}=$ broad singlet, and so on], $\mathrm{d}=$ doublet, $\mathrm{t}=$ triplet, $\mathrm{m}=$ multiplet, $\mathrm{dd}=$ doublet of doublets, $\mathrm{dt}=$ doublet of triplets, and $\mathrm{br}=$ broad signal) and coupling constants are given in $\mathrm{Hz}$. Compounds $4,{ }^{[1]} \mathbf{6}^{[2]}$ and $\mathbf{7}^{[3]}$ have been prepared as previously described.

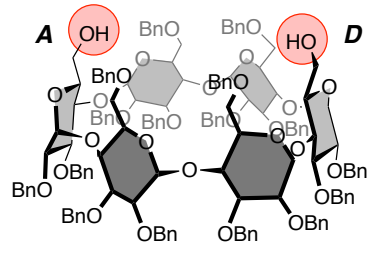

4

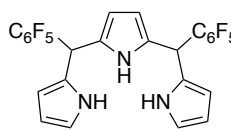

6

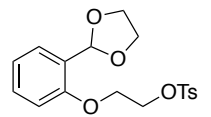

7

[1] (a) Pearce, A. J.; Sinaÿ, P. Angew. Chem. Int. Ed. 2000, 39, 3610-3612. (b) Lecourt, T.; Herault, A.; Pearce, A. J.; Sollogoub, M.; Sinä̈, P. Chem. Eur. J. 2004, 10, 2960-2971.

[2] Tanaka, T.; Osuka, A. Chem. Rev. 2017, 117, 2584-2640.

[3] Ménand, M.; Sollogoub, M.; Boitrel, B.; Le Gac, S. Angew. Chem. Int. Ed. 2016, 55, 297-301. 


\section{Synthesis and description}

\subsection{Cyclodextrin-bis acetal 8}

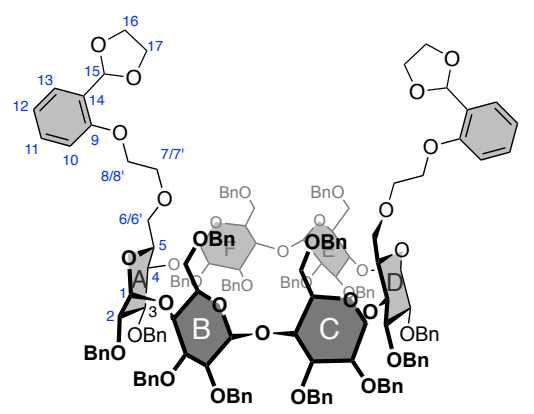

$430 \mathrm{mg}(0.177 \mathrm{mmol})$ of cyclodextrin diol 4 were dissolved in anhydrous THF under inert atmosphere. $57 \mathrm{mg}$ of $\mathrm{NaH}(60 \% \mathrm{w} / \mathrm{w}$ in oil, $1.426 \mathrm{mmol}$ ) were added. After 10 minutes of stirring, $389 \mathrm{mg}$ (1.069 $\mathrm{mmol}$ ) of the acetal derivative $\mathbf{7}$ were added and the mixture was refluxed overnight. Upon cooling to room temperature, few drops of water were added and THF was removed under reduced pressure. The residue was subjected to column chromatography purification on a 15 micron silica gel $\left(\mathrm{CH}_{2} \mathrm{Cl}_{2}\right.$ /ethyl acetate 99:1), leading to $437 \mathrm{mg}$ of cyclodextrin-bis acetal 8 as a white solid $(88 \%) .{ }^{1} \mathbf{H}$ NMR (500MHz, CDCl, $298 \mathrm{~K}): \delta 3.39-3.48(\mathrm{~m}, 8 \mathrm{H}, \mathrm{H} 2[\mathrm{~A}, \mathrm{~B}, \mathrm{C}, \mathrm{D}, \mathrm{E}, \mathrm{F}], \mathrm{H} 6[\mathrm{~A}, \mathrm{D}]), 3.51\left(\mathrm{~d}_{\mathrm{b}},{ }^{2} \mathrm{~J}=10.5 \mathrm{~Hz}, 2 \mathrm{H}\right.$, $\mathrm{H} 6[\mathrm{C}, \mathrm{F}]), 3.54-3.61(\mathrm{~m}, 4 \mathrm{H}, \mathrm{H} 6[\mathrm{~B}, \mathrm{E}], \mathrm{H7}[\mathrm{A}, \mathrm{D}]) ; 3.75\left(\mathrm{dt},{ }^{2} \mathrm{~J}_{7,7^{\prime}}=10.6 \mathrm{~Hz},{ }^{3} \mathrm{~J}_{7^{\prime}, 8}=5.4 \mathrm{~Hz}, 2 \mathrm{H}, \mathrm{H} 7^{\prime}[\mathrm{A}, \mathrm{D}]\right) ; 3.80-$ 3.88 (m, 6H, H5[A,D], H16[A,D], H17[A,D]); 3.89-4.06 (m, 2OH, H4[A,B,C,D,E,F], H5[B,C,E,F], H6' [C,F], $\left.H 8[A, D], H 8^{\prime}[A, D], H 16^{\prime}[A, D], H 17^{\prime}[A, D]\right) ; 4.06-4.16\left(m, 10 H, H 3[A, B, C, D, E, F], H 6^{\prime}[A, B, D, E]\right), 4.32$ (d, ${ }^{2} J$ $\left.=12.1 \mathrm{~Hz}, 2 \mathrm{H}, \mathrm{CH} \mathrm{H}^{\mathrm{Bn}-6}[\mathrm{~B}, \mathrm{E}]\right), 4.34\left(\mathrm{~d},{ }^{2} J=12.1 \mathrm{~Hz}, 2 \mathrm{H}, \mathrm{CH} \mathrm{H}^{\mathrm{Bn}-6}[\mathrm{C}, \mathrm{F}]\right), 4.39-4.46\left(\mathrm{~m}, 8 \mathrm{H}, \mathrm{CH} \mathrm{H}^{\mathrm{Bn}-2}[\mathrm{~B}, \mathrm{C}, \mathrm{E}, \mathrm{F}]\right.$, $\left.\mathrm{CH} H^{\mathrm{Bn}-6}[\mathrm{~B}, \mathrm{C}, \mathrm{E}, \mathrm{F}]\right), 4.50\left(\mathrm{~d},{ }^{2} \mathrm{~J}=12.5 \mathrm{~Hz}, 2 \mathrm{H}, \mathrm{CH} H^{\mathrm{Bn}-2}[\mathrm{~A}, \mathrm{D}]\right), 4.83\left(\mathrm{~d},{ }^{2} J=11.0 \mathrm{~Hz}, 2 \mathrm{H}, 2 \times \mathrm{CHH}^{\mathrm{Bn}-3}\right), 4.84(\mathrm{~d}$, $\left.{ }^{2} \mathrm{~J}=10.9 \mathrm{~Hz}, 2 \mathrm{H}, 2 \times \mathrm{CHH}^{\mathrm{Bn}-3}\right), 4.85\left(\mathrm{~d},{ }^{2} \mathrm{~J}=11.0 \mathrm{~Hz}, 2 \mathrm{H}, 2 \times \mathrm{CHH}^{\mathrm{Bn}-3}\right), 5.05\left(\mathrm{~d},{ }^{3} \mathrm{~J}_{1,2}=3.4 \mathrm{~Hz}, 2 \mathrm{H}, \mathrm{H} 1[\mathrm{C}, \mathrm{F}]\right)$, $5.07\left(\mathrm{~d},{ }^{3} \mathrm{~J}_{1,2}=3.4 \mathrm{~Hz}, 2 \mathrm{H}, \mathrm{H} 1[\mathrm{~B}, \mathrm{E}]\right), 5.09-5.16\left(\mathrm{~m}, 6 \mathrm{H}, \mathrm{H} 1[\mathrm{~A}, \mathrm{D}], 4 \times \mathrm{CH} H^{\mathrm{Bn}-3}\right), 5.21\left(\mathrm{~d},{ }^{2} \mathrm{~J}=11.0 \mathrm{~Hz}, 2 \mathrm{H}\right.$, $\left.2 \times \mathrm{CH}^{\mathrm{Bn}-3}\right), 6.17(\mathrm{~s}, 2 \mathrm{H}, \mathrm{H} 15[\mathrm{~A}, \mathrm{D}]), 6.82\left(\mathrm{~d},{ }^{3} \mathrm{~J}_{10,11}=8.1 \mathrm{~Hz}, 2 \mathrm{H}, \mathrm{H} 10[\mathrm{~A}, \mathrm{D}]\right), 6.96\left(\mathrm{~d},{ }^{3} \mathrm{~J}_{11,12}={ }^{3} \mathrm{~J}_{12,13}=7.4\right.$ $\mathrm{Hz}, 2 \mathrm{H}, \mathrm{H} 12[\mathrm{~A}, \mathrm{D}]), 7.06-7.28\left(\mathrm{~m}, 82 \mathrm{H}, \mathrm{H} 11[\mathrm{~A}, \mathrm{D}], 80 \times \mathrm{HAr}^{\mathrm{Bn}}\right), 7.49\left(\mathrm{dd},{ }^{3} \mathrm{~J}_{12,13}=7.4 \mathrm{~Hz},{ }^{4} \mathrm{~J}_{11,13}=1.8 \mathrm{~Hz}, 2 \mathrm{H}\right.$, $\mathrm{H} 13[\mathrm{~A}, \mathrm{D}])$. HRMS (ESI-TOF, $\mathrm{m} / \mathrm{z}$ ) for $\mathrm{C}_{170} \mathrm{H}_{180} \mathrm{O}_{36} \mathrm{Na}[\mathrm{M}+\mathrm{Na}]^{+}$, calculated 2820.21465, found 2820.2115. 


\subsection{Cyclodextrin-bis aldehyde 5}

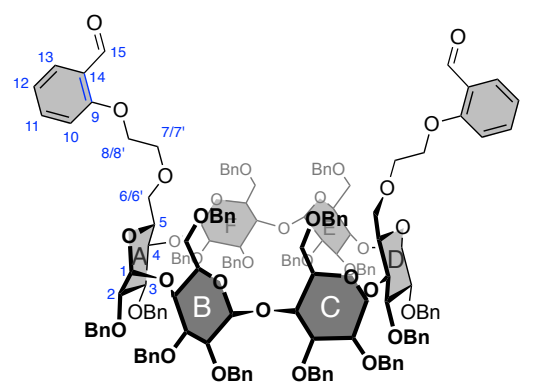

Under inert atmosphere, $278 \mu \mathrm{L}(3,72 \mathrm{mmol})$ of TFA were added to a solution of $348 \mathrm{mg}(0.124 \mathrm{mmol})$ of the cyclodextrin bis-acetal 8 in $20 \mathrm{~mL}$ of $\mathrm{CH}_{2} \mathrm{Cl}_{2}$, and the mixture was stirred at room temperature for 1 hour. The organic phase was washed four times with water, then dried over $\mathrm{MgSO}_{4}$ and concentrated under reduced pressure. $325 \mathrm{mg}$ of cyclodextrin bis-aldehyde derivative 5 were obtained as a white solid (96\%). ${ }^{1} \mathbf{H}$ NMR $\left(500 \mathrm{MHz}, \mathrm{CDCl}_{3}, 298 \mathrm{~K}\right): \delta 3.40-3.49(\mathrm{~m}, 8 \mathrm{H}, \mathrm{H} 2[\mathrm{~A}, \mathrm{~B}, \mathrm{C}, \mathrm{D}, \mathrm{E}, \mathrm{F}], \mathrm{H} 6[\mathrm{~A}, \mathrm{D}])$; $3.53\left(\mathrm{~d}_{\mathrm{b}},{ }^{2} \mathrm{~J}=10.9 \mathrm{~Hz}, 2 \mathrm{H}, \mathrm{H} 6[\mathrm{~B}, \mathrm{E}]\right) ; 3.58-3.65(\mathrm{~m}, 4 \mathrm{H}, \mathrm{H} 7[\mathrm{~A}, \mathrm{D}], \mathrm{H} 6[\mathrm{C}, \mathrm{F}]) ; 3.71\left(\mathrm{dt},{ }^{2} \mathrm{~J}_{7,7^{\prime}}=10.4 \mathrm{~Hz},{ }^{3} \mathrm{~J}_{7,8 / 8^{\prime}}=\right.$ $\left.5.0 \mathrm{~Hz}, 2 \mathrm{H}, \mathrm{H} 7^{\prime}[A, D]\right) ; 3.86\left(\mathrm{~d}_{\mathrm{b}}, 3 \mathrm{~J}=9.1 \mathrm{~Hz}, 2 \mathrm{H}, \mathrm{H} 5[\mathrm{~A}, \mathrm{D}]\right) ; 3.89-4.18(\mathrm{~m}, 26 \mathrm{H}, \mathrm{H} 3[\mathrm{~A}, \mathrm{~B}, \mathrm{C}, \mathrm{D}, \mathrm{E}, \mathrm{F}]$, $\left.H 4[A, B, C, D, E, F], H 5[B, C, E, F], H 6^{\prime}[A, B, C, D, E, F], H 8[A, D], H 8^{\prime}[A, D]\right), 4.32-4.47\left(m, 20 H, 18 \times H^{B n}\right) ; 4.53(d$, $\left.{ }^{2} J=12.5 \mathrm{~Hz}, 2 \mathrm{H}, 2 \times \mathrm{H}^{\mathrm{Bn}}\right) ; 4.83\left(\mathrm{~d},{ }^{2} J=10.8 \mathrm{~Hz}, 2 \mathrm{H}, 2 \times \mathrm{H}^{\mathrm{Bn}}\right) ; 4.85-4.90\left(\mathrm{~m}, 4 \mathrm{H}, 4 \times \mathrm{H}^{\mathrm{Bn}}\right) ; 4.93\left(\mathrm{~d},{ }^{3} J_{1,2}=3.3 \mathrm{~Hz}\right.$, $2 \mathrm{H}, \mathrm{H} 1[\mathrm{C}, \mathrm{F}]) ; 5.03\left(\mathrm{~d},{ }^{3} \mathrm{~J}_{1,2}=3.4 \mathrm{~Hz}, 2 \mathrm{H}, \mathrm{H} 1[\mathrm{~B}, \mathrm{E}]\right) ; 5.09\left(\mathrm{~d},{ }^{2} \mathrm{~J}=11.1 \mathrm{~Hz}, 2 \mathrm{H}, 2 \times \mathrm{H}^{\mathrm{Bn}}\right) ; 5.15\left(\mathrm{~d},{ }^{2} \mathrm{~J}=10.9 \mathrm{~Hz}\right.$, $\left.2 \mathrm{H}, 2 \times \mathrm{H}^{\mathrm{Bn}}\right) ; 5.22\left(\mathrm{~d},{ }^{3} J_{1,2}=3.5 \mathrm{~Hz}, 2 \mathrm{H}, \mathrm{H} 1[\mathrm{~A}, \mathrm{D}]\right) ; 5.27\left(\mathrm{~d},{ }^{2} \mathrm{~J}=11.0 \mathrm{~Hz}, 2 \mathrm{H}, 2 \times \mathrm{H}^{\mathrm{Bn}}\right) ; 6.89\left(\mathrm{~d},{ }^{3} J_{10,11}=8.4 \mathrm{~Hz}\right.$, $2 \mathrm{H}, \mathrm{H} 10[\mathrm{~A}, \mathrm{D}]) ; 7.00\left(\mathrm{t},{ }^{3} \mathrm{~J}_{11,12}={ }^{3} \mathrm{~J}_{12,13}=7.5 \mathrm{~Hz}, 2 \mathrm{H}, \mathrm{H} 12[\mathrm{~A}, \mathrm{D}]\right) ; 7.06-7.29\left(\mathrm{~m}, 80 \mathrm{H}, 80 \times \mathrm{H}^{\mathrm{Ar}}\right) ; 7.46$ (ddd, $\left.{ }^{3} J_{10,11}=8.4,{ }^{3} J_{11,12}=7.5 \mathrm{~Hz},{ }^{4} J_{11,13}=1.8 \mathrm{~Hz}, 2 \mathrm{H}, \mathrm{H} 11[\mathrm{~A}, \mathrm{D}]\right) ; 7.80\left(\mathrm{dd},{ }^{3} J_{12,13}=7.5 \mathrm{~Hz},{ }^{4} J_{11,13}=1.8 \mathrm{~Hz}, 2 \mathrm{H}\right.$, $\mathrm{H} 11[\mathrm{~A}, \mathrm{D}]) ; 10.46$ (s, 2H, H15[A,D]). ${ }^{13} \mathrm{C}$ NMR (125MHz, CDCl $\left.3,298 \mathrm{~K}\right): \delta 67.89$ (C8[A,D]), 69.25 (C6[B,E]), 69.39 (C6[C,F]), 69.70 (C7[A,D]), 70.26 (C6[A,D]), 71.59 (C5[A,D]), 71.63 (C5[B,E]), 71.86 (C5[C,F]), 72.73 $\left(\mathrm{CH}_{2}{ }^{\mathrm{Bn}}\right), 73.00\left(\mathrm{CH}_{2}{ }^{\mathrm{Bn}}\right), 73.05\left(\mathrm{CH}_{2}{ }^{\mathrm{Bn}}\right), 73.46\left(\mathrm{CH}_{2}{ }^{\mathrm{Bn}}\right), 73.56\left(\mathrm{CH}_{2}{ }^{\mathrm{Bn}}\right), 75.36\left(\mathrm{CH}_{2}{ }^{\mathrm{Bn}}\right), 75.90\left(\mathrm{CH}_{2}{ }^{\mathrm{Bn}}\right), 75.95$ $\left(\mathrm{CH}_{2}{ }^{\mathrm{Bn}}\right.$ ), 78.67 (C4[B,E]), 78.81 (C2), 79.33 (C2), 79.53 (C2), 80.16 (C4[C,F]), 80.39 (C4[A,D]), 80.85 (C3), 80.97 (C3), 81.18 (C3), 98.96 (C1[B,E]), 99.02 (C1[A,D]), 99.23 (C1[C,F]), 113.03 (C10[A,D]), 121.17 (C12[A,D]), 125.23 (C14[A,D]), 127.04 (CAr $\left.{ }^{\mathrm{Bn}}\right), 127.10\left(\mathrm{CAr}^{\mathrm{Bn}}\right), 127.20\left(\mathrm{CAr}^{\mathrm{Bn}}\right), 127.47\left(\mathrm{CAr}^{\mathrm{Bn}}\right), 127.50$ $\left(\mathrm{CAr}^{\mathrm{Bn}}\right), 127.54\left(\mathrm{CAr}^{\mathrm{Bn}}\right), 127.58\left(\mathrm{CAr}^{\mathrm{Bn}}\right), 127.63\left(\mathrm{CAr}^{\mathrm{Bn}}\right), 127.70\left(\mathrm{CAr}^{\mathrm{Bn}}\right), 127.76\left(\mathrm{CAr}^{\mathrm{Bn}}\right), 127.79\left(\mathrm{CAr}^{\mathrm{Bn}}\right)$, $127.93\left(\mathrm{CAr}^{\mathrm{Bn}}\right), 128.05\left(\mathrm{CAr}^{\mathrm{Bn}}\right), 128.09\left(\mathrm{CAr}^{\mathrm{Bn}}\right), 128.11\left(\mathrm{CAr}^{\mathrm{Bn}}\right), 128.14\left(\mathrm{CAr}^{\mathrm{Bn}}\right), 128.16\left(\mathrm{CAr}^{\mathrm{Bn}}\right), 128.24$ $\left(C^{B n}\right), 128.30\left(C^{B} r^{B n}\right), 128.42\left(C^{B}{ }^{B n}\right), 128.48$ (C13[A,D]), 135.94 (C11[A,D]), $138.20\left(C_{q} A r^{B n}\right), 138.31$ $\left(C_{q} A r^{B n}\right), 138.39\left(C_{q} A r^{B n}\right), 138.49\left(C_{q} A r^{B n}\right), 138.61\left(C_{q} A r^{B n}\right), 139.44\left(C_{q} A r^{B n}\right), 139.47\left(C_{q} A r^{B n}\right), 139.54$ $\left(C_{a} A r^{B n}\right), 161.25$ (C9[A,D]), 189.61 (C15[A,D]). HRMS (ESI-TOF, $\left.m / z\right)$ for $\mathrm{C}_{166} \mathrm{H}_{172} \mathrm{O}_{34} \mathrm{Na}[\mathrm{M}+\mathrm{Na}]^{+}$, calculated 2732.16222, found 2732.1592. 


\section{$2.3{ }^{\mathrm{R}}[26] 1$ and $\mathrm{M}[28] 2 / \mathrm{M}[28] 3$}

Under inert atmosphere, $2.5 \mathrm{~g}(0.92 \mathrm{mmol})$ of cyclodextrin-bis aldehyde 5 and $1.0 \mathrm{~g}(1.79 \mathrm{mmol})$ of tripyrromethane 6 were solubilized in $55 \mathrm{~mL}$ of anhydrous $\mathrm{CH}_{2} \mathrm{Cl}_{2}$. At $0^{\circ} \mathrm{C}, 11.5 \mu \mathrm{L}(0.177 \mathrm{mmol})$ of MSA were added and the reactional mixture was stirred for $1 \mathrm{~h} 30$ at this temperature. DDQ $(0.9 \mathrm{~g}, 4.0 \mathrm{mmol})$ was then added and the solution was allowed to reach room temperature. After $20 \mathrm{~min}$, an excess of TEA (1.4 mL, $10.4 \mathrm{mmol}$ ) was added and the solvent was removed under reduced pressure. The residue was subjected twice to silica gel chromatography purification (cyclohexane/ethylacetate 9:1 to 6:4), leading to $106 \mathrm{mg}$ of ${ }^{\mathrm{M}}[\mathbf{2 8}] \mathbf{2} /{ }^{\mathrm{M}}[\mathbf{2 8}] \mathbf{3}$ mixture as a blue solid (3.0\% yield) and $81 \mathrm{mg}$ of ${ }^{\mathrm{R}}[\mathbf{2 6}] \mathbf{1}$ as a purple solid ( $2.3 \%$ yield).

\section{${ }^{\mathrm{R}}[26] 1:$}

${ }^{1} \mathrm{H}$ NMR $\left(500 \mathrm{MHz}, \mathrm{CDCl}_{3}, 298 \mathrm{~K}\right): \delta-2.95\left(\mathrm{~d}, 2 \mathrm{H},{ }^{3} \mathrm{~J}=4.4 \mathrm{~Hz}, \pi 2 / 5\right)$; $2.70\left(\mathrm{~d}, 2 \mathrm{H},{ }^{3} \mathrm{~J}=4.4 \mathrm{~Hz}, \pi 2 / 5\right) ;-1.58(\mathrm{sb}, 2 \mathrm{H}, 2 \times \mathrm{NH}) ; 1.90\left(\mathrm{~d}, 2 \mathrm{H},{ }^{2} \mathrm{~J}=\right.$ $11.5 \mathrm{~Hz}, \mathrm{H} 6[\mathrm{~A}, \mathrm{D}])$. 1.99-2.07 (m, 4H, CHH $\left.{ }^{\mathrm{CD}}[\mathrm{A}, \mathrm{D}], \mathrm{H} 6[\mathrm{C}, \mathrm{F}]\right), 2.13$ (br, $\left.2 \mathrm{H}, \mathrm{CH} H^{\mathrm{CD}}[\mathrm{A}, \mathrm{D}]\right), 2.48\left(\mathrm{~d}, 2 \mathrm{H},{ }^{2} \mathrm{~J}=11.9 \mathrm{~Hz}, \mathrm{CHH}^{\mathrm{Bn}-6}[\mathrm{C}, \mathrm{F}]\right) ; 2.54(\mathrm{~d}, 2 \mathrm{H}$, $\left.{ }^{2} J=11.9 \mathrm{~Hz}, \mathrm{CH} H^{\mathrm{Bn}-6}[\mathrm{C}, \mathrm{F}]\right) ; 2.65\left(\mathrm{~d}_{\mathrm{b}}, 2 \mathrm{H},{ }^{2} J=11.1 \mathrm{~Hz}, \mathrm{H}^{\prime}[\mathrm{C}, \mathrm{F}]\right) ; 2.77$ (d, $\left.2 \mathrm{H},{ }^{2} J=11.2 \mathrm{~Hz}, \mathrm{H} 6[\mathrm{~B}, \mathrm{E}]\right) ; 2.88\left(\mathrm{~d}_{\mathrm{b}}, 2 \mathrm{H},{ }^{2} \mathrm{~J}=10.5 \mathrm{~Hz}, \mathrm{H} 6^{\prime}[\mathrm{A}, \mathrm{D}]\right)$; 2.96 (br, 2H, H5[A,D]); 2.98-3.03 (m, 4H, H2[C,F], H5[B,E]), 3.06 (dd, $\left.2 \mathrm{H},{ }^{3} J_{1,2}=2.9,3_{2,3}^{3}=9.8 \mathrm{~Hz}, \mathrm{H} 2[\mathrm{~A}, \mathrm{D}]\right) ; 3.19\left(\mathrm{~d}_{\mathrm{b}}, 2 \mathrm{H},{ }^{3} \mathrm{~J}=9.7 \mathrm{~Hz}\right.$, $\mathrm{H} 5[\mathrm{C}, \mathrm{F}]) ; 3.26-3.34\left(\mathrm{~m}, 4 \mathrm{H}, \mathrm{H} 2[\mathrm{~B}, \mathrm{E}], \mathrm{H} 6^{\prime}[\mathrm{B}, \mathrm{E}]\right), 3.44\left(\mathrm{t}, 2 \mathrm{H},{ }^{3} \mathrm{~J}_{3,4}={ }^{3} \mathrm{~J}_{4,5}\right.$ $=9.2 \mathrm{~Hz}, \mathrm{H} 4[\mathrm{~A}, \mathrm{D}]) ; 3.45\left(\mathrm{t}, 2 \mathrm{H},{ }^{3} J_{3,4}={ }^{3} J_{4,5}=9.2 \mathrm{~Hz}, \mathrm{H} 4[\mathrm{C}, \mathrm{F}]\right) ; 3.59(\mathrm{br}$, $\left.2 \mathrm{H}, \mathrm{CHH}^{\mathrm{H}}[\mathrm{A}, \mathrm{D}]\right) ; 3.63\left(\mathrm{t}, 2 \mathrm{H},{ }^{3} \mathrm{~J}_{2,3}={ }^{3} \mathrm{~J}_{3,4}=9.2 \mathrm{~Hz}, \mathrm{H} 3[\mathrm{~A}, \mathrm{D}]\right) ; 3.68(\mathrm{t}, 2 \mathrm{H}$,

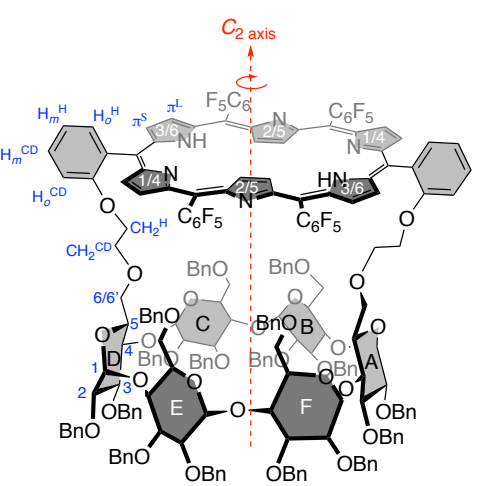
$\left.{ }^{3} J_{2,3}={ }^{3} J_{3,4}=9.2 \mathrm{~Hz}, \mathrm{H} 3[\mathrm{C}, \mathrm{F}]\right) ; 3.72-3.82\left(\mathrm{~m}, 6 \mathrm{H}, \mathrm{CH} H^{H}[\mathrm{~A}, \mathrm{D}], \mathrm{H} 3[\mathrm{~B}, \mathrm{E}], \mathrm{H} 4[\mathrm{~B}, \mathrm{E}]\right) ; 3.90\left(\mathrm{~d}, 2 \mathrm{H},{ }^{2} \mathrm{~J}=12.1 \mathrm{~Hz}\right.$, $\left.\mathrm{CH}^{\mathrm{Bn}-6}[\mathrm{~B}, \mathrm{E}]\right) ; 4.05-4.10\left(\mathrm{~m}, 4 \mathrm{H}, 2 \times \mathrm{H}^{\mathrm{Bn}}, \mathrm{CH} H^{\mathrm{Bn}-6}[\mathrm{~B}, \mathrm{E}]\right) ; 4.13\left(\mathrm{~d}, 2 \mathrm{H},{ }^{2} J=12.2 \mathrm{~Hz}, 2 \times \mathrm{H}^{\mathrm{Bn}}\right) ; 4.17\left(\mathrm{~d}, 2 \mathrm{H},{ }^{3} J_{1,2}\right.$ $=3.2 \mathrm{~Hz}, \mathrm{H} 1[\mathrm{C}, \mathrm{F}]) ; 4.24\left(\mathrm{~d}, 2 \mathrm{H},{ }^{2} \mathrm{~J}=12.2 \mathrm{~Hz}, 2 \times \mathrm{H}^{\mathrm{Bn}}\right) ; 4.26\left(\mathrm{~d}, 2 \mathrm{H},{ }^{2} \mathrm{~J}=12.5 \mathrm{~Hz}, 2 \times \mathrm{H}^{\mathrm{Bn}}\right) ; 4.32\left(\mathrm{~d}, 2 \mathrm{H},{ }^{2} \mathrm{~J}=\right.$ $\left.12.5 \mathrm{~Hz}, 2 \times \mathrm{H}^{\mathrm{Bn}}\right) ; 4.40\left(\mathrm{~d}, 2 \mathrm{H},{ }^{3} \mathrm{~J}_{1,2}=3.0 \mathrm{~Hz}, \mathrm{H} 1[\mathrm{~A}, \mathrm{D}]\right) ; 4.51\left(\mathrm{~d}, 2 \mathrm{H},{ }^{2} \mathrm{~J}=11.3 \mathrm{~Hz}, 2 \times \mathrm{H}^{\mathrm{Bn}}\right) ; 4.54\left(\mathrm{~d}, 2 \mathrm{H},{ }^{2} \mathrm{~J}=\right.$ $\left.12.6 \mathrm{~Hz}, 2 \times \mathrm{H}^{\mathrm{Bn}}\right) ; 4.57\left(\mathrm{~d}, 2 \mathrm{H},{ }^{2} \mathrm{~J}=12.2 \mathrm{~Hz}, 2 \times \mathrm{H}^{\mathrm{Bn}}\right) ; 4.60\left(\mathrm{~d}, 2 \mathrm{H},{ }^{2} \mathrm{~J}=12.2 \mathrm{~Hz}, 2 \times \mathrm{H}^{\mathrm{Bn}}\right) ; 4.65\left(\mathrm{~d}, 2 \mathrm{H},{ }^{2} J=10.7\right.$ $\left.\mathrm{Hz}, 2 \times \mathrm{H}^{\mathrm{Bn}}\right) ; 4.84-4.95\left(\mathrm{~m}, 8 \mathrm{H}, 2 \times \mathrm{H}_{m}{ }^{\mathrm{Bn}-6}[\mathrm{C}, \mathrm{F}], \mathrm{H}_{p}{ }^{\mathrm{Bn}-6}[\mathrm{C}, \mathrm{F}], 2 \times \mathrm{H}^{\mathrm{Bn}}\right) ; 5,20\left(\mathrm{~d}, 2 \mathrm{H},{ }^{2} \mathrm{~J}=10.7 \mathrm{~Hz}, 2 \times \mathrm{H}^{\mathrm{Bn}}\right) ; 5.25(\mathrm{~d}$, $\left.4 \mathrm{H},{ }^{3} \mathrm{~J}=7.3 \mathrm{~Hz}, 2 \times \mathrm{H}_{0}^{\mathrm{Bn}-6}[\mathrm{C}, \mathrm{F}]\right) ; 5.33\left(\mathrm{~d}, 2 \mathrm{H},{ }^{3} \mathrm{~J}_{1,2}=3.8 \mathrm{~Hz}, \mathrm{H} 1[\mathrm{~B}, \mathrm{E}]\right) ; 6.75-7.17\left(\mathrm{~m}, 70 \mathrm{H}, 70 \times \mathrm{H}_{\mathrm{Ar}}{ }^{\mathrm{Bn}}\right) ; 7.20(\mathrm{~d}$, $\left.2 \mathrm{H},{ }^{3} \mathrm{~J}=8.4 \mathrm{~Hz}, \mathrm{H}_{0}{ }^{\mathrm{CD}}[\mathrm{A}, \mathrm{D}]\right) ; 7.71\left(\mathrm{t}, 2 \mathrm{H},{ }^{3} \mathrm{~J}=7.6 \mathrm{~Hz}, \mathrm{H}_{m}{ }^{\mathrm{H}}[\mathrm{A}, \mathrm{D}]\right) ; 7.92\left(\mathrm{dt}, 2 \mathrm{H},{ }^{4} \mathrm{~J}=1.8,{ }^{3} \mathrm{~J}=8.3 \mathrm{H}_{m}{ }^{\mathrm{CD}}[\mathrm{A}, \mathrm{D}]\right)$; $8.93\left(\mathrm{dd}, 2 \mathrm{H},{ }^{4} \mathrm{~J}=1.8,{ }^{3} \mathrm{~J}=7.3 \mathrm{~Hz}, \mathrm{H}_{o}^{\mathrm{H}}[\mathrm{A}, \mathrm{D}]\right) ; 9.02\left(\mathrm{~d}, 2 \mathrm{H},{ }^{3} \mathrm{~J}=4.7 \mathrm{~Hz}, \pi^{\mathrm{S}} 1 / 4\right) ; 9.12\left(\mathrm{~d}, 2 \mathrm{H}, 3^{3} \mathrm{~J}=4.8 \mathrm{~Hz}\right.$, $\left.\pi^{\mathrm{S}} 3 / 6\right) ; 9.20\left(\mathrm{~d}, 2 \mathrm{H},{ }^{3} \mathrm{~J}=4.7 \mathrm{~Hz}, \pi^{\mathrm{L}} 1 / 4\right) ; 9.23\left(\mathrm{~d}, 2 \mathrm{H},{ }^{3} \mathrm{~J}=4.8 \mathrm{~Hz}, \pi^{\mathrm{L}} 3 / 6\right) .{ }^{19} \mathrm{~F}$ NMR $\left(376 \mathrm{MHz}, \mathrm{CDCl}_{3}, 298 \mathrm{~K}\right)$ : $\delta$-133.99 (t, 2F, J = 23.3 Hz, $\mathrm{F}_{o}$ ), $-135.07\left(\mathrm{t}, 2 \mathrm{~F}, J=24.4 \mathrm{~Hz}, \mathrm{~F}_{o}\right),-139.16\left(\mathrm{~d}, 2 \mathrm{~F}, J=22.5 \mathrm{~Hz}, \mathrm{~F}_{o}\right),-139.30$ $\left(\mathrm{d}, 2 \mathrm{~F}, J=23.7 \mathrm{~Hz}, \mathrm{~F}_{o}\right),-153.11\left(\mathrm{t}, 2 \mathrm{~F}, J=20.7 \mathrm{~Hz}, \mathrm{~F}_{p}\right),-153.21\left(\mathrm{t}, 2 \mathrm{~F}, J=20.4 \mathrm{~Hz}, \mathrm{~F}_{p}\right),-162.77\left(\mathrm{~m}, 2 \mathrm{~F}, \mathrm{~F}_{m}\right)$, -162.82 (m, 2F, $\left.F_{m}\right),-163.18\left(m, 2 F, F_{m}\right),-163.61\left(m, 2 F, F_{m}\right) .{ }^{13} \mathrm{C}$ from 2D HSQC $\left(500 \mathrm{MHz}, \mathrm{CDCl}_{3}, 300\right.$ $\mathrm{K}): \delta 66.2\left(\mathrm{CH}_{2}{ }^{\mathrm{H}}[\mathrm{A}, \mathrm{D}]\right) ; 67.6$ (C6[C,F]); 68.4 (C6[B,E]); $68.8\left(\mathrm{CH}_{2}{ }^{\mathrm{CD}}[\mathrm{A}, \mathrm{D}]\right) ; 69.3$ (C6[A,D]); 70.2 (C5[C,F]); 71.1 (C5[A,D]); $71.2(\mathrm{C} 5[\mathrm{~B}, \mathrm{E}]) ; 71.6\left(\mathrm{CH}_{2}{ }^{\mathrm{Bn}-6}[\mathrm{C}, \mathrm{F}]\right) ; 72.3\left(2 \times \mathrm{CH}_{2}{ }^{\mathrm{Bn}}\right) ; 72.7\left(2 \times \mathrm{CH}_{2}{ }^{\mathrm{Bn}}\right) ; 72.8\left(2 \times \mathrm{CH}_{2}{ }^{\mathrm{Bn}}\right) ; 73.2$ $\left(2 \times \mathrm{CH}_{2}{ }^{\mathrm{Bn}}\right) ; 74.1\left(2 \times \mathrm{CH}_{2}{ }^{\mathrm{Bn}}\right) ; 75.0(\mathrm{C} 4[\mathrm{C}, \mathrm{F}]) ; 75.9\left(2 \times \mathrm{CH}_{2}{ }^{\mathrm{Bn}}\right) ; 76.3\left(2 \times \mathrm{CH}_{2}{ }^{\mathrm{Bn}}\right) ; 77.8(\mathrm{C} 2[\mathrm{~B}, \mathrm{E}]) ; 78.8$ (C2[A,D]); 79.7 (C2[C,F]); 80.1 (C4[A,D]); 80.3 (C3[A,D]); 80.7 (C3[B,E], C4[B,E]); 81.3 (C3[C,F]); 97.7 (C1[A,D]); 98.2

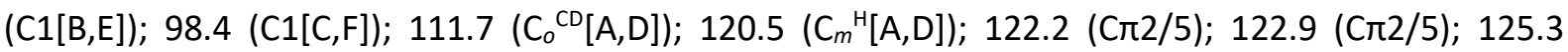
$\left(2 \times \mathrm{C}_{o}{ }^{\mathrm{Bn}-6}[\mathrm{C}, \mathrm{F}]\right) ; 125.8\left(\mathrm{C}_{p}^{\mathrm{Bn}-6}[\mathrm{C}, \mathrm{F}]\right) ; 126.0\left(2 \times \mathrm{C}_{m}{ }^{\mathrm{Bn}-6}[\mathrm{C}, \mathrm{F}]\right) ; 126.5-128.2\left(70 \times \mathrm{CAr}^{\mathrm{Bn}}\right) ; 131.4\left(\mathrm{C}_{m}^{\mathrm{CD}}[\mathrm{A}, \mathrm{D}]\right) ;$

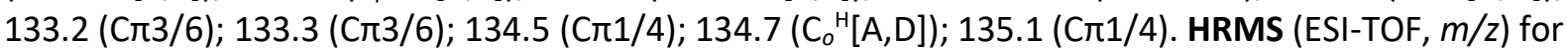
$\mathrm{C}_{218} \mathrm{H}_{185} \mathrm{~N}_{6} \mathrm{O}_{32} \mathrm{~F}_{20}[\mathrm{M}+\mathrm{H}]^{+}$, calculated 3778.27086, found 3778.2699. UV-vis $\left(\mathrm{CH}_{2} \mathrm{Cl}_{2}\right): \lambda\left(\varepsilon, \mathrm{M}^{-1} . \mathrm{cm}^{-1}\right)=577$ $\left(1.42 \times 10^{5}\right), 615\left(5.33 \times 10^{4}\right), 731\left(1.38 \times 10^{4}\right), 788\left(1.01 \times 10^{4}\right), 893\left(6.98 \times 10^{3}\right), 1020\left(5.19 \times 10^{3}\right) \mathrm{nm}$. 
The different fluxional regime of the hybrid parts (cyclodextrin/linkers/hexaphyrin) at $330 \mathrm{~K}$ hampered the assignment of the bridged glucopyranose units due to a lack of NOE correlations between the linkers and both the cyclodextrin and the hexaphyrin. Hence, the glucopyranose units (for each isomer) are described using labels I, II and III following a counterclockwise rotation on the primary face. The full assignment of the hexaphyrin protons was not possible due to a broadening and a strong overlapping of the signals in particular for the $\beta$-pyrrolic protons.

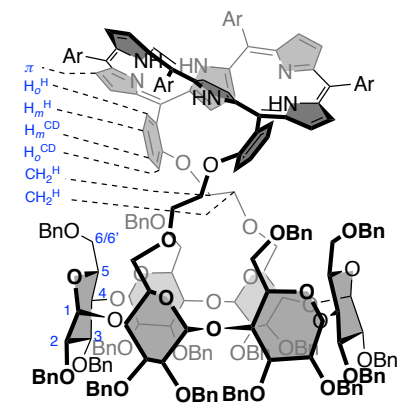

$\mathrm{M}_{[28] 2}$

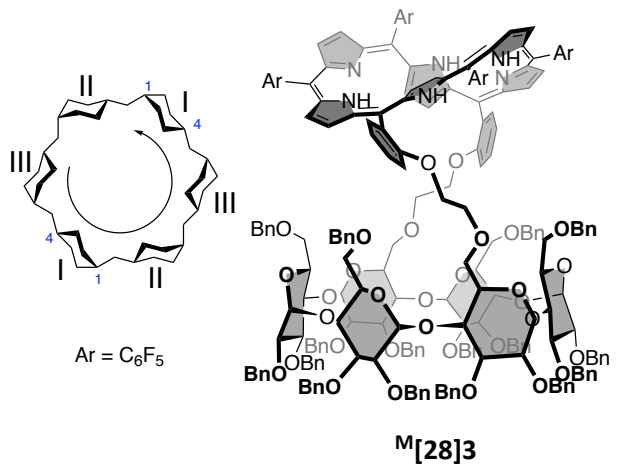

$\mathrm{M}[28] 3$

partial description of ${ }^{\mathrm{M}}[\mathbf{2 8}] \mathbf{2}$ labelled [2] / ${ }^{\mathrm{M}}[\mathbf{2 8}] \mathbf{3}$ labelled [3] :

${ }^{1} \mathrm{H} \mathrm{NMR}\left(500 \mathrm{MHz}, \mathrm{CDCl}_{3}, 330 \mathrm{~K}\right): \delta 2.48\left(\mathrm{br}, 2 \mathrm{H}, 2 \times \mathrm{CHH}^{\mathrm{CD}} 3\right) ; 2.55-2.65\left(\mathrm{~m}, 4 \mathrm{H}, 2 \times \mathrm{CH}^{\mathrm{CD}}[3], 2 \times \mathrm{CHH}^{\mathrm{CD}}[2]\right)$; $2.75\left(\mathrm{~d}, 2 \mathrm{H},{ }^{2} \mathrm{~J}=12.3 \mathrm{~Hz}, 2 \times \mathrm{H} 6[\mathrm{I}-3]\right) ; 2.78\left(\mathrm{~d}, 2 \mathrm{H},{ }^{2} \mathrm{~J}=12.1 \mathrm{~Hz}, 2 \times \mathrm{H} 6[\mathrm{II}-2]\right) ; 2.84\left(\mathrm{~d}, 2 \mathrm{H},{ }^{2} \mathrm{~J}=12.3 \mathrm{~Hz}\right.$, $2 \times \mathrm{H} 6[\mathrm{II}-3]) ; 3.02\left(\mathrm{br}, 2 \mathrm{H}, 2 \times \mathrm{CHH}^{\mathrm{CD}}[2]\right) ; 3.05\left(\mathrm{dd}, 2 \mathrm{H},{ }^{3} \mathrm{I}_{1,2}=3.3,{ }^{3} J_{2,3}=9.7 \mathrm{~Hz}, 2 \times \mathrm{H} 2[\mathrm{II}-2]\right) ; 3.09\left(\mathrm{~d}, 2 \mathrm{H},{ }^{2} \mathrm{~J}\right.$ $=10.9 \mathrm{~Hz}, 2 \times \mathrm{HG} 6[\mathrm{III}-2]) ; 3.07-3.17\left(\mathrm{~m}, 4 \mathrm{H}, 2 \times \mathrm{CH}_{2}{ }^{\mathrm{H}}[3]\right) ; 3.17$ (dd, $\left.2 \mathrm{H},{ }^{3} \mathrm{~J}_{1,2}=3.1,{ }^{3} \mathrm{~J}_{2,3}=9.8 \mathrm{~Hz}, 2 \times \mathrm{H} 2[\mathrm{II}-3]\right)$; $3.26\left(\mathrm{dd}, 2 \mathrm{H},{ }^{3} \mathrm{~J}_{1,2}=3.2,{ }^{3} \mathrm{~J}_{2,3}=9.6 \mathrm{~Hz}, 2 \times \mathrm{H} 2[\mathrm{III}-3]\right) ; 3.40-3.59(\mathrm{~m}, 22 \mathrm{H}, 2 \times \mathrm{H} 2[\mathrm{I}-2], 2 \times \mathrm{H} 2[\mathrm{III}-2], 2 \times \mathrm{H} 2[\mathrm{I}-3]$, $\left.2 \times \mathrm{H} 5[\mathrm{I}-3], 2 \times \mathrm{H} 5[\mathrm{III}-3], 2 \times \mathrm{H} 6[\mathrm{I}-3], 2 \times \mathrm{H}^{\prime}[\mathrm{II}-3], 2 \times \mathrm{H}^{\prime}[\mathrm{III}-3], 2 \times \mathrm{CH}_{2}{ }^{\mathrm{H}}[2], 2 \times \mathrm{CHH}^{\mathrm{Bn}-6}[3]\right) ; 3.59-3.82(\mathrm{~m}, 24 \mathrm{H}$, $2 \times \mathrm{H} 4\left[\mathrm{II}-2\right.$ ] $, 2 \times \mathrm{H} 4[\mathrm{II}-3], 2 \times \mathrm{H} 4[\mathrm{III}-3], 2 \times \mathrm{H} 5[\mathrm{I}-2], 2 \times \mathrm{H} 5\left[\mathrm{II}-2\right.$ ], $2 \times \mathrm{H} 5$ [III-2], $2 \times \mathrm{H} 5[\mathrm{II}-3], 2 \times \mathrm{H} 6[\mathrm{II} 2], 2 \times \mathrm{H} 6^{\prime}[\mathrm{II}-2$ ], $\left.2 \times \mathrm{H}^{\prime}[\mathrm{III}-2], 2 \times \mathrm{CHH}^{\mathrm{Bn}-6}[3], 2 \times \mathrm{CHH}^{\mathrm{Bn}-6}[2]\right) ; 3.84-4.07(\mathrm{~m}, 16 \mathrm{H}, 2 \times \mathrm{H} 3[\mathrm{II}-2], 2 \times \mathrm{H} 3[\mathrm{III}-2], 2 \times \mathrm{H} 3[\mathrm{I}-3], 2 \times \mathrm{H} 3[\mathrm{II}-$ 3], $\left.2 \times \mathrm{H} 3[\mathrm{III}-3], 2 \times \mathrm{H} 4[\mathrm{III}-2], 2 \times \mathrm{H} 4[\mathrm{II}-3], 2 \times \mathrm{H} 6^{\prime}[\mathrm{I}-3]\right) ; 4.12\left(\mathrm{t}_{\mathrm{b}}, 2 \mathrm{H},{ }^{3} \mathrm{~J}_{2,3}=3_{3,4}^{3}=8.6 \mathrm{~Hz}, 2 \times \mathrm{H} 3[\mathrm{I}-2]\right) ; 4.15-4.43$ $\left(\mathrm{m}, 16 \mathrm{H}, 2 \times \mathrm{H} 1[\mathrm{II}-2], 2 \times \mathrm{H} 4[\mathrm{I}-2], 2 \times \mathrm{H}^{\prime}[\mathrm{I}-2], 2 \times \mathrm{CHH}^{\mathrm{Bn}-6}[2], 8 \times \mathrm{H}^{\mathrm{Bn}}\right) ; 4.44-4.60\left(\mathrm{~m}, 13 \mathrm{H}, 2 \times \mathrm{H} 1[\mathrm{II}-3], 11 \times \mathrm{H}^{\mathrm{Bn}}\right)$; 4.62-4.82 (m, 31H, $\left.2 \times \mathrm{H} 1[\mathrm{III}-3], 29 \times \mathrm{H}^{\mathrm{Bn}}\right) ; 5.04\left(\mathrm{~d}, 1 \mathrm{H},{ }^{3} J_{1,2}=3.3 \mathrm{~Hz}, 2 \times \mathrm{H} 1[\mathrm{III}-2]\right) ; 5.11\left(\mathrm{~d}, 2 \mathrm{H},{ }^{2} J=10.9 \mathrm{~Hz}\right.$, $\left.2 \times \mathrm{H}^{\mathrm{Bn}}\right) ; 5.16\left(\mathrm{~d}, 2 \mathrm{H},{ }^{2} \mathrm{~J}=10.8 \mathrm{~Hz}, 2 \times \mathrm{H}^{\mathrm{Bn}}\right) ; 5.24-5.30\left(\mathrm{~m}, 4 \mathrm{H}, 4 \times \mathrm{H}^{\mathrm{Bn}}\right) ; 5.51\left(\mathrm{~d}, 1 \mathrm{H},{ }^{3} \mathrm{~J}_{1,2}=3.7 \mathrm{~Hz}, 2 \times \mathrm{H} 1[\mathrm{I}-2]\right)$; $5.53\left(\mathrm{~d}, 1 \mathrm{H},{ }^{3} J_{1,2}=3.6 \mathrm{~Hz}, 2 \times \mathrm{H} 1[\mathrm{I}-3]\right) ; 5.93\left(\mathrm{br}, 2 \mathrm{H}, 2 \times \mathrm{H}_{p}{ }^{\mathrm{Bn}-6}[2]\right) ; 6.06\left(\mathrm{br}, 2 \mathrm{H}, 2 \times \mathrm{H}_{p}{ }^{\mathrm{Bn}-6}[3]\right) ; 6.12-6.21(\mathrm{~m}$, $\left.8 \mathrm{H}, 4 \times \mathrm{H}_{m}{ }^{\mathrm{Bn}-6}[2], 4 \times \mathrm{H}_{m}^{\mathrm{Bn}-6}[3]\right) ; 6.33\left(\mathrm{~d}_{\mathrm{b}}, 4 \mathrm{H},{ }^{2} \mathrm{~J}=7.5 \mathrm{~Hz}, 4 \times \mathrm{H}_{o}^{\mathrm{Bn}-6}[3]\right) ; 6.45-6.50\left(\mathrm{~m}, 6 \mathrm{H}, 2 \times \mathrm{H}^{\pi}[2], 4 \times \mathrm{H}_{o}{ }^{\mathrm{Bn}-}\right.$ $\left.{ }^{6}[2]\right) ; 6.69\left(\mathrm{~d}_{\mathrm{b}}, 2 \mathrm{H},{ }^{3} \mathrm{~J}=4.6 \mathrm{~Hz}, 2 \times \mathrm{H}^{\pi}[2]\right) ; 6.81\left(\mathrm{~d}, 2 \mathrm{H},{ }^{3} \mathrm{~J}=8.0 \mathrm{~Hz}, 2 \times \mathrm{H}_{0}{ }^{\mathrm{CD}}[2]\right) ; 6.85\left(\mathrm{~d}_{\mathrm{b}}, 2 \mathrm{H}, 3^{3} \mathrm{~J}=4.8 \mathrm{~Hz}\right.$, $\left.2 \times \mathrm{H}^{\pi}[3]\right) ; 6.87\left(\mathrm{~d}, 2 \mathrm{H},{ }^{3} \mathrm{~J}=8.3 \mathrm{~Hz}, 2 \times \mathrm{H}_{o}^{\mathrm{CD}}[\mathbf{3}]\right) ; 6.89-7.51\left(\mathrm{~m}, 158 \mathrm{H}, 2 \times \mathrm{H}_{m}{ }^{\mathrm{CD}}[2], 2 \times \mathrm{H}_{m}{ }^{\mathrm{H}}[\mathbf{2}], 2 \times \mathrm{H}_{m}{ }^{\mathrm{CD}}[3]\right.$, $\left.2 \times \mathrm{H}_{m}{ }^{\mathrm{H}}[3], 150 \times \mathrm{H}_{\mathrm{Ar}}{ }^{\mathrm{Bn}}\right) ; 7.68\left(\mathrm{~d}, 2 \mathrm{H},{ }^{3} \mathrm{~J}=7.6 \mathrm{~Hz}, 2 \times \mathrm{H}_{o}{ }^{\mathrm{H}}[2]\right) ; 7.77\left(\mathrm{~d}, 2 \mathrm{H},{ }^{3} \mathrm{~J}=7.6 \mathrm{~Hz}, 2 \times \mathrm{H}_{o}{ }^{\mathrm{H}}[3]\right) .{ }^{13} \mathrm{C}$ from 2D

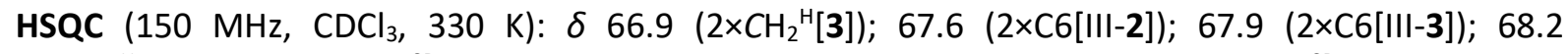
$\left(2 \times \mathrm{CH}_{2}{ }^{\mathrm{H}}[2]\right) ; 69.1\left(2 \times \mathrm{CH}_{2}{ }^{\mathrm{CD}}[3]\right) ; 69.4-70.4$ (2×C6[I-3], $\left.2 \times \mathrm{C} 6[1-2], 2 \times \mathrm{C} 6[\mathrm{II}-3], 2 \times \mathrm{CH}_{2}{ }^{\mathrm{CD}}[2]\right) ; 70.6-71.8$ (2xC5[I-2], $2 \times \mathrm{C} 5[\mathrm{II}-3], 2 \times \mathrm{C} 5[\mathrm{II}-2], 2 \times \mathrm{C} 5[\mathrm{II}-3], 2 \times \mathrm{C} 5[\mathrm{III}-2], 2 \times \mathrm{C} 5[\mathrm{III}-3]) ; 73.2\left(2 \times \mathrm{CH}_{2}{ }^{\mathrm{Bn}-6}[2]\right) ; 73.3\left(2 \times \mathrm{CH}_{2}{ }^{\mathrm{Bn}-}\right.$ $\left.{ }^{6}[3]\right) ; 74.3-74.4(2 \times \mathrm{C} 4[\mathrm{II}-2], 2 \times \mathrm{C} 4[\mathrm{II}-3]) ; 71.9-74.6\left(24 \times \mathrm{CH}_{2}{ }^{\mathrm{Bn}}\right) 75.4\left(2 \times \mathrm{CH}_{2}{ }^{\mathrm{Bn}}\right), 76.3\left(2 \times \mathrm{CH}_{2}{ }^{\mathrm{Bn}}\right), 77.9-79.6$

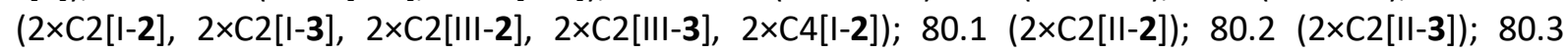

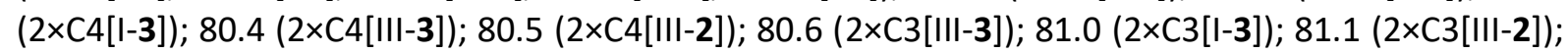

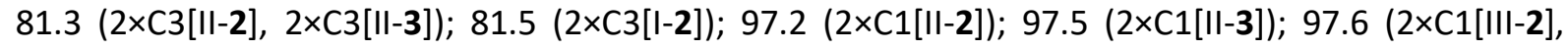
$2 \times \mathrm{C} 1[1-2]) ; 97.9(2 \times \mathrm{C} 1[\mathrm{III}-3]) ; 98.0(2 \times \mathrm{C} 1[\mathrm{I}-3]) ; 113.7\left(2 \times \mathrm{C}_{o}^{\mathrm{CD}}[3]\right) ; 114.1\left(2 \times \mathrm{C}_{o}^{\mathrm{CD}}[2]\right) ; 121.0\left(2 \times \mathrm{C}_{m}{ }^{\mathrm{H}}[\mathbf{2}]\right.$, $\left.2 \times \mathrm{C}_{m}{ }^{\mathrm{H}}[3]\right) ; 125.6-129.6\left(160 \times \mathrm{C}_{\mathrm{Ar}}{ }^{\mathrm{Bn}}, 8 \times \mathrm{C} \pi\right) ; 131.0\left(2 \times \mathrm{C}_{m}{ }^{\mathrm{CD}}[2], 2 \times \mathrm{C}_{m}{ }^{\mathrm{CD}}[3]\right) ; 133.5\left(2 \times \mathrm{C}_{o}{ }^{\mathrm{H}}[2]\right) ; 133.6$ $\left(2 \times \mathrm{C}_{0}{ }^{\mathrm{H}}[3]\right)$. HRMS (ESI-TOF, $\left.m / z\right)$ for $\mathrm{C}_{218} \mathrm{H}_{186} \mathrm{~N}_{6} \mathrm{O}_{32} \mathrm{~F}_{20}\left[\mathrm{M}^{\cdot}\right]^{+}$, calculated 3779.27868 , found 3779.2769 . UV-vis $\left(1: 1{ }^{\mathrm{M}}[28] 2 /{ }^{\mathrm{M}}[28] 3, \mathrm{CH}_{2} \mathrm{Cl}_{2}\right): \lambda\left(\varepsilon, \mathrm{M}^{-1} \cdot \mathrm{cm}^{-1}\right)=310\left(3.07 \times 10^{4}\right), 397\left(3.65 \times 10^{4}\right), 491\left(4.97 \times 10^{4}\right)$, $555\left(5.91 \times 10^{4}\right), 601\left(1.90 \times 10^{5}\right), 635\left(6.71 \times 10^{4}\right), 767\left(1.32 \times 10^{4}\right), 859\left(7.37 \times 10^{3}\right), 895\left(6.45 \times 10^{3}\right)$, $1005\left(1.92 \times 10^{3}\right) \mathrm{nm}$. 
3 Reduction/oxidation procedures and descriptions

3.1 General scheme of the red/ox interconversion of all HCD hybrids

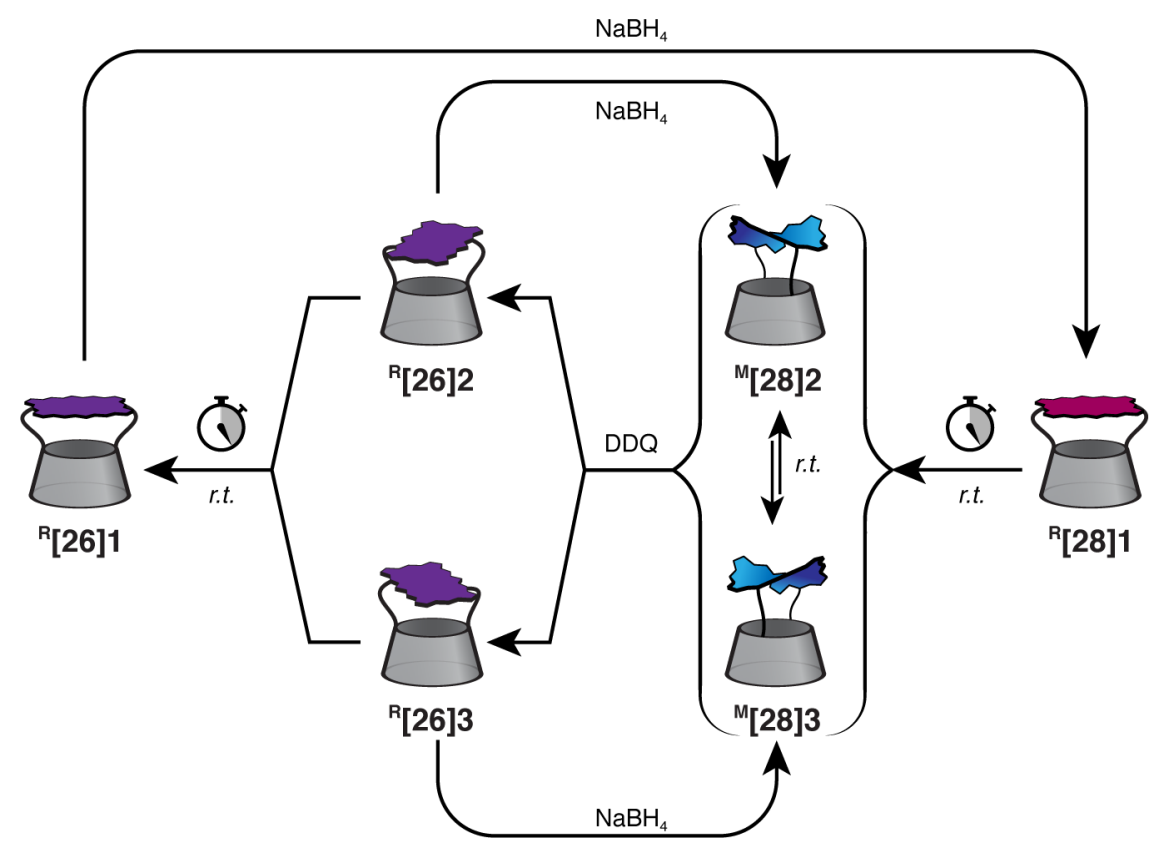




\section{$3.2{ }^{\mathrm{R}}[\mathbf{2 8}] 1$ by reduction of ${ }^{\mathrm{R}}[\mathbf{2 6}] 1$}

In a standard NMR tube, $5 \mathrm{mg}(1.27 \mu \mathrm{mol})$ of ${ }^{\mathrm{R}}[26] 1$ were dissolved in $0.5 \mathrm{~mL}$ of THF $d_{8}$, and $2 \mathrm{mg}$ (53 $\mu \mathrm{mol}$ ) of $\mathrm{NaBH}_{4}$ were subsequently added. The NMR tube solution was sonicated at room temperature for $30 \mathrm{~min} . \mathrm{A}^{1} \mathrm{H}$ NMR spectrum was then recorded, showing complete formation of ${ }^{\mathrm{R}}[\mathbf{2 8}] \mathbf{1}$. At room temperature, ${ }^{\mathrm{R}}[28] 1$ slowly converts to the mixture of ${ }^{\mathrm{M}}[\mathbf{2 8}] \mathbf{2} /{ }^{\mathrm{M}}[28] 3$, rendering full characterization difficult.

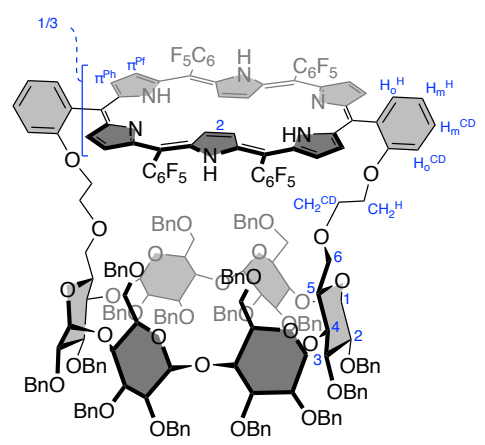

${ }^{R}[28] 1:{ }^{1} \mathrm{H}$ NMR (500 MHz, THFd $8,298 \mathrm{~K}$, partial description): $\delta 3.50$ (dd, $2 \mathrm{H},{ }^{3}{ }_{1,2}=9.8,3.4 \mathrm{~Hz}, 2 \times \mathrm{H} 2$ ), $3.57(\mathrm{br}, 2 \mathrm{H}, 2 \times \mathrm{H} 2), 3.66\left(\mathrm{dd}, 2 \mathrm{H},{ }^{3} J_{1,2}=9.9,3.2 \mathrm{~Hz}, 2 \times \mathrm{H} 2\right), 3.89\left(\mathrm{~d}_{\mathrm{b}}, 2 \mathrm{H},{ }^{3} J=4.1 \mathrm{~Hz}, 2 \times \pi\left[1^{\mathrm{Pf}}\right]\right), 3.91\left(\mathrm{~d}_{\mathrm{b}}\right.$, $\left.2 \mathrm{H},{ }^{3} \mathrm{~J}=4.5 \mathrm{~Hz}, 2 \times \pi\left[3^{\mathrm{Pf}}\right]\right), 3.95\left(\mathrm{~d}, 2 \mathrm{H},{ }^{2} J_{6,6^{\prime}}=11.2 \mathrm{~Hz}, 2 \times \mathrm{H6}\right), 4.15\left(\mathrm{~d}, 2 \mathrm{H},{ }^{2} \mathrm{~J}_{6,6^{\prime}}=11.0 \mathrm{~Hz}, 2 \times \mathrm{H6}\right), 4.17-4.33$ $\left(\mathrm{m}, 22 \mathrm{H}, 6 \times \mathrm{H} 3,6 \times \mathrm{H} 4,4 \times \mathrm{H} 5,2 \times \mathrm{CHH}^{\mathrm{H}}, 4 \times \mathrm{H} 6\right), 4.34-4.60\left(\mathrm{~m}, 16 \mathrm{H}, 2 \times \mathrm{H} 5,2 \times \mathrm{H} 6,2 \times \mathrm{CHH}^{\mathrm{H}}, 2 \times \mathrm{CH}_{2}{ }^{\mathrm{CD}}, 6 \times \mathrm{H}^{\mathrm{Bn}}\right)$, 4.65-4.73 (m, $\left.6 \mathrm{H}, 2 \times \pi\left[1^{\mathrm{Ph}}\right], 4 \times \mathrm{H}^{\mathrm{Bn}}\right), 4.76-4.82\left(\mathrm{~m}, 6 \mathrm{H}, 2 \times \pi\left[3^{\mathrm{Ph}}\right], 4 \times \mathrm{H}^{\mathrm{Bn}}\right), 4.92\left(\mathrm{~d}, 2 \mathrm{H},{ }^{2} \mathrm{~J}=10.7 \mathrm{~Hz}, 2 \times \mathrm{H}^{\mathrm{Bn}}\right)$, 4.94-5.02 (m, 10H, $\left.2 \times H 6,8 \times H^{B n}\right), 5.04\left(d_{b}, 2 \mathrm{H},{ }^{3} J_{1,2}=3.8 \mathrm{~Hz}, 2 \times H 1\right), 5.22\left(\mathrm{~d}, 2 \mathrm{H},{ }^{2} \mathrm{~J}=11.7 \mathrm{~Hz}, 2 \times C H \mathrm{H}^{B n-}\right.$ $\left.{ }^{6 i n}\right), 5.27\left(\mathrm{~d}, 2 \mathrm{H},{ }^{2} \mathrm{~J}=11.7 \mathrm{~Hz}, 2 \times \mathrm{CHH}^{\mathrm{Bn}-6 \mathrm{in}}\right), 5.29\left(\mathrm{~d}_{\mathrm{b}}, 2 \mathrm{H},{ }^{3} \mathrm{~J}_{1,2}=3.5 \mathrm{~Hz}, 2 \times \mathrm{H} 1\right), 5.43\left(\mathrm{~d}, 2 \mathrm{H},{ }^{2} \mathrm{~J}=11.3 \mathrm{~Hz}\right.$, $\left.2 \times \mathrm{H}^{\mathrm{Bn}}\right), 5.59\left(\mathrm{~d}, 2 \mathrm{H},{ }^{2} \mathrm{~J}=10.6 \mathrm{~Hz}, 2 \times \mathrm{H}^{\mathrm{Bn}}\right), 5.68\left(\mathrm{~d}_{\mathrm{b}}, 2 \mathrm{H},{ }^{3} \mathrm{~J}_{1,2}=3.6 \mathrm{~Hz}, 2 \times \mathrm{H} 1\right), 6.05\left(\mathrm{~d}_{\mathrm{b}}, 2 \mathrm{H},{ }^{3} J=7.6 \mathrm{~Hz}, 2 \times \mathrm{H}_{o}{ }^{\mathrm{H}}\right)$, $6.59\left(\mathrm{t}, 2 \mathrm{H},{ }^{3} \mathrm{~J}=7.4 \mathrm{~Hz}, 2 \times \mathrm{H}_{m}{ }^{\mathrm{H}}\right), 6.88-7.52\left(\mathrm{~m}, 74 \mathrm{H}, 2 \times \mathrm{H}_{m}{ }^{\mathrm{CD}}, 2 \times \mathrm{H}_{o}{ }^{\mathrm{CD}}, 70 \times \mathrm{HAr}^{\mathrm{Bn}}\right), 8.44\left(\mathrm{~d}_{\mathrm{b}}, 4 \mathrm{H},{ }^{3} \mathrm{~J}=7.3 \mathrm{~Hz}\right.$, $\left.4 \times \mathrm{H}_{o}^{\text {Bn-6in }}\right), 8.65\left(\mathrm{~d}_{\mathrm{b}}, 2 \mathrm{H},{ }^{3} \mathrm{~J}=7.3 \mathrm{~Hz}, 2 \times \mathrm{H}_{p}{ }^{\mathrm{Bn}-6 \mathrm{in}}\right), 8.68\left(\mathrm{~d}_{\mathrm{b}}, 4 \mathrm{H},{ }^{3} \mathrm{~J}=7.3 \mathrm{~Hz}, 4 \times \mathrm{H}_{m}{ }^{\mathrm{Bn}-6 \mathrm{in}}\right), 14.67\left(\mathrm{~s}_{\mathrm{b}}, 2 \mathrm{H}, 2 \times \pi[2]\right)$, $14.91\left(\mathrm{~s}_{\mathrm{b}}, 2 \mathrm{H}, 2 \times \pi^{\prime}[2]\right) .{ }^{13} \mathrm{C}$ from 2D HSQC $\left(150 \mathrm{MHz}, \mathrm{THF}-\mathrm{d}_{8}, 298 \mathrm{~K}\right): \delta 67.3\left(2 \times \mathrm{CH}_{2}{ }^{\mathrm{H}}\right), 69.9(2 \times \mathrm{C} 6), 70.4$ (2×C6), $70.5\left(2 \times \mathrm{CH}_{2}{ }^{\mathrm{Bn}}\right), 71.1(2 \times \mathrm{C} 6), 71.7(2 \times \mathrm{C} 5), 72.3(2 \times \mathrm{C} 5), 72.5-74.0\left(2 \times \mathrm{C}^{2}, 2 \times \mathrm{CH}_{2}{ }^{\mathrm{CD}}, 6 \times \mathrm{CH}_{2}{ }^{\mathrm{Bn}}\right), 74.6$ $\left(2 \times \mathrm{CH}_{2}{ }^{\mathrm{Bn}-6 \mathrm{in}}\right), 74.7\left(2 \times \mathrm{CH}_{2}{ }^{\mathrm{Bn}}\right), 76.0\left(2 \times \mathrm{CH}_{2}{ }^{\mathrm{Bn}}\right), 76.5\left(2 \times \mathrm{CH}_{2}{ }^{\mathrm{Bn}}\right), 77.3(2 \times \mathrm{C} 4), 79.0(2 \times \mathrm{C} 2), 79.9(2 \times \mathrm{C} 2), 81.3$ $(2 \times \mathrm{C} 2)$ 81.4-82.4 (6×C3, 4×C4), $98.9(2 \times \mathrm{C} 1), 99.5(2 \times \mathrm{C} 1), 99.9(2 \times \mathrm{C} 1), 112.3\left(2 \times \mathrm{C}_{o}^{\mathrm{CD}}\right), 16.9\left(2 \times \mathrm{C} \pi\left[1^{\mathrm{Pf}}\right]\right.$, $\left.2 \times \mathrm{C} \pi\left[2^{\mathrm{Pf}}\right]\right), 120.5\left(2 \times \mathrm{C}_{m}{ }^{\mathrm{H}}\right), 126.4-129.4\left(2 \times \mathrm{C}_{m}{ }^{\mathrm{CD}}, 70 \times \mathrm{CH}^{\mathrm{Ar}}\right), 129.6\left(2 \times \mathrm{C} \pi\left[1^{\mathrm{Ph}}\right]\right), 129.7\left(4 \times \mathrm{C}_{0}^{\mathrm{Bn}-6 \mathrm{6in}}\right), 129.8$ $\left(2 \times C \pi\left[2^{\mathrm{Ph}}\right]\right), 130.2\left(2 \times \mathrm{C}_{p}^{\mathrm{Bn}-6 \mathrm{in}}\right), 130.4\left(4 \times \mathrm{C}_{m}{ }^{\mathrm{Bn}-6 \mathrm{in}}\right), 131.0\left(4 \times \mathrm{C}_{o}^{\mathrm{H}}\right), 137.4(2 \times \mathrm{C} \pi[3]), 137.3(2 \times \mathrm{C} \pi[3]) .{ }^{19} \mathrm{~F}$ NMR (376 MHz, THF- $\left.d_{8}, 298 \mathrm{~K}\right): \delta-138.05\left(\mathrm{~d}, 2 \mathrm{~F}, J=25.2 \mathrm{~Hz}, 2 \times \mathrm{F}_{o}\right),-138.16\left(\mathrm{~d}, J=24.8 \mathrm{~Hz}, 2 \times \mathrm{F}_{o}\right),-139.82$

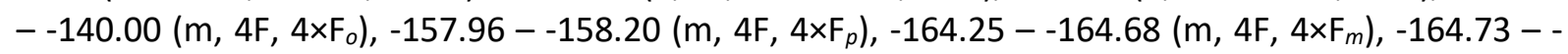
$164.96\left(\mathrm{~m}, 4 \mathrm{~F}, 4 \times \mathrm{F}_{m}\right)$. UV-vis (THF, broad spectrum contaminated with $\left.{ }^{\mathrm{M}}[28] 2 /{ }^{\mathrm{M}}[28] 3\right): \lambda=530 \mathrm{~nm}$. 


\section{$3.3{ }^{\mathrm{R}}[26] 2$ and ${ }^{\mathrm{R}}[26] 3$ by oxidation of ${ }^{\mathrm{M}}[28] 2 /{ }^{\mathrm{M}}[28] 3$}

Under inert atmosphere, $50 \mathrm{mg}(0.013 \mathrm{mmol})$ of ${ }^{\mathrm{M}}[28] 2 /{ }^{\mathrm{M}}[28] 3$ (1:1) were dissolved in anhydrous $\mathrm{CH}_{2} \mathrm{Cl}_{2}(7 \mathrm{~mL})$, then $4.5 \mathrm{mg}(0.019 \mathrm{mmol})$ of DDQ were added. After 1 hour of stirring at room temperature, the reaction was quenched by the addition of $5 \mu \mathrm{L}$ of TEA $(0.039 \mathrm{mmol})$, and the solvent was evaporated under reduced pressure. The residue was subjected to column chromatography purification (cyclohexane $/ \mathrm{CH}_{2} \mathrm{Cl}_{2}$ /ethyl acetate $50: 50: 1$ ), leading to $24.0 \mathrm{mg}$ of ${ }^{\mathrm{R}}[\mathbf{2 6}] \mathbf{2}$ as a purple solid (49\% yield) and $23.6 \mathrm{mg}$ of ${ }^{\mathrm{R}}[\mathbf{2 6}] \mathbf{3}$ as a purple solid (48\% yield).

${ }^{\mathrm{R}}[26] 2:$

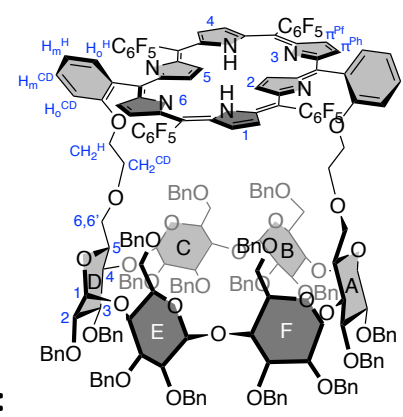

${ }^{1} \mathrm{H}$ NMR (500 MHz, CDCl $\left.3,283 \mathrm{~K}\right): \delta:-3.71\left(\mathrm{~d}, 2 \mathrm{H},{ }^{3} \mathrm{~J}=4.3 \mathrm{~Hz}, \pi[2,5]\right),-3.42\left(\mathrm{~d}, 2 \mathrm{H},{ }^{3} \mathrm{~J}=4.3 \mathrm{~Hz}, \pi^{\prime}[2,5]\right)$, $-2.91(\mathrm{~s}, 2 \mathrm{H}, 2 \times \mathrm{NH}), 1.52\left(\mathrm{~d}, 2 \mathrm{H},{ }^{2} \mathrm{~J}_{6,6^{\prime}}=12.6 \mathrm{~Hz}, \mathrm{H} 6[\mathrm{C}, \mathrm{F}]\right), 1.82\left(\mathrm{~d}, 2 \mathrm{H},{ }^{2} \mathrm{~J}_{6,6^{\prime}}=12.1 \mathrm{~Hz}, \mathrm{H} 6[\mathrm{~A}, \mathrm{D}]\right), 2.16(\mathrm{br}$, $\left.2 \mathrm{H}, \mathrm{CHH}^{\mathrm{CD}}[\mathrm{A}, \mathrm{D}]\right), 2.31\left(\mathrm{~d}, 2 \mathrm{H},{ }^{2} J=12.2 \mathrm{~Hz}, \mathrm{CHH}^{\mathrm{Bn}-6}[\mathrm{C}, \mathrm{F}]\right), 2.35-2.45\left(\mathrm{~m}, 4 \mathrm{H}, \mathrm{H} 6^{\prime}[\mathrm{C}, \mathrm{F}], \mathrm{CH}{ }^{\mathrm{Bn}-6}[\mathrm{C}, \mathrm{F}]\right), 2.69-$ $2.79\left(\mathrm{~m}, 6 \mathrm{H}, \mathrm{H} 6[\mathrm{~B}, \mathrm{E}], \mathrm{H} \mathrm{G}^{\prime}[\mathrm{A}, \mathrm{D}], \mathrm{CH} \mathrm{H}^{\mathrm{CD}}[\mathrm{A}, \mathrm{D}]\right), 2.87\left(\mathrm{~d}_{\mathrm{b}}, 2 \mathrm{H},{ }^{3} \mathrm{~J}_{4,5}=9.6 \mathrm{~Hz}, \mathrm{H} 5[\mathrm{~A}, \mathrm{D}]\right), 2.97\left(\mathrm{~d}_{\mathrm{b}}, 2 \mathrm{H},{ }^{3} \mathrm{~J}_{4,5}=9.4\right.$ $\mathrm{Hz}, \mathrm{H} 5[\mathrm{~B}, \mathrm{E}]), 3.02\left(\mathrm{~d}_{\mathrm{b}}, 2 \mathrm{H},{ }^{3} \mathrm{~J}_{4,5}=9.9 \mathrm{~Hz}, \mathrm{H} 5[\mathrm{C}, \mathrm{F}]\right), 3.07$ (dd, $\left.2 \mathrm{H},{ }^{3} \mathrm{~J}_{1,2}=3.0,{ }^{3} \mathrm{~J}_{2,3}=9.7 \mathrm{~Hz}, \mathrm{H} 2[\mathrm{~A}, \mathrm{D}]\right), 3.10$ (dd, $\left.2 \mathrm{H}, 3_{1,2}^{3}=3.0,3_{2,3}^{3}=9.8 \mathrm{~Hz}, \mathrm{H} 2[\mathrm{C}, \mathrm{F}]\right), 3.29$ (dd, $\left.2 \mathrm{H},{ }^{3} J_{1,2}=3.7,3_{2,3}^{3}=9.8 \mathrm{~Hz}, \mathrm{H} 2[\mathrm{~B}, \mathrm{E}]\right), 3.41-3.49(\mathrm{~m}$, $\left.6 \mathrm{H}, \mathrm{H} 4[\mathrm{~A}, \mathrm{D}], \mathrm{H} 4[\mathrm{C}, \mathrm{F}], \mathrm{H} 6^{\prime}[\mathrm{B}, \mathrm{E}]\right), 3.52\left(\mathrm{t}_{\mathrm{b}}, 2 \mathrm{H},{ }^{3} \mathrm{~J}=7.2 \mathrm{~Hz}, \mathrm{H}_{p}^{\mathrm{Bn}-6}[\mathrm{C}, \mathrm{F}]\right), 3.56\left(\mathrm{t}, 2 \mathrm{H},{ }^{3} \mathrm{~J}_{2,3}={ }^{3} \mathrm{~J}_{3,4}=9.2 \mathrm{~Hz}\right.$, $\mathrm{H} 3[\mathrm{~A}, \mathrm{D}]), 3.70\left(\mathrm{t}, 2 \mathrm{H},{ }^{3} \mathrm{~J}_{2,3}={ }^{3} \mathrm{~J}_{3,4}=9.3 \mathrm{~Hz}, \mathrm{H} 3[\mathrm{C}, \mathrm{F}]\right), 3.78\left(\mathrm{dd}, 2 \mathrm{H},{ }^{3} J_{2,3}=9.8,{ }^{3} J_{3,4}=7.7 \mathrm{~Hz}, \mathrm{H} 3[\mathrm{~B}, \mathrm{E}]\right), 3.93-$ $4.02\left(\mathrm{~m}, 8 \mathrm{H}, \mathrm{H} 4[\mathrm{~B}, \mathrm{E}], \mathrm{CHH}^{\mathrm{Bn}-6}[\mathrm{~B}, \mathrm{E}], \mathrm{CH}_{2}{ }^{\mathrm{H}}[\mathrm{A}, \mathrm{D}]\right), 4.08\left(\mathrm{br}, 4 \mathrm{H}, 2 \times \mathrm{H}_{m}{ }^{\mathrm{Bn}-6}[\mathrm{C}, \mathrm{F}]\right), 4.20\left(\mathrm{~d}, 2 \mathrm{H},{ }^{2} \mathrm{~J}=12.3 \mathrm{~Hz}\right.$, $\left.2 \times H^{B n}\right), 4.26-4.38\left(m, 12 \mathrm{H}, \mathrm{H} 1[\mathrm{~A}, \mathrm{D}], \mathrm{CH} H^{\mathrm{Bn}-6}[\mathrm{~B}, \mathrm{E}], 8 \times \mathrm{H}^{\mathrm{Bn}}\right), 4.46\left(\mathrm{~d}, 2 \mathrm{H},{ }^{3} \mathrm{~J}_{1,2}=3.4 \mathrm{~Hz}, \mathrm{H} 1[\mathrm{C}, \mathrm{F}]\right), 4.48(\mathrm{~d}, 4 \mathrm{H}$, $\left.{ }^{2} J=11.4 \mathrm{~Hz}, 4 \times \mathrm{H}^{\mathrm{Bn}}\right), 4.57-4.67\left(\mathrm{~m}, 10 \mathrm{H}, 2 \times \mathrm{H}_{o}^{\mathrm{Bn}-6}[\mathrm{C}, \mathrm{F}], 6 \times \mathrm{H}^{\mathrm{Bn}}\right), 4.87\left(\mathrm{~d}, 2 \mathrm{H},{ }^{2} J=10.8 \mathrm{~Hz}, 2 \times \mathrm{H}^{\mathrm{Bn}}\right), 5.22(\mathrm{~d}$, $\left.2 \mathrm{H},{ }^{2} J=10.6 \mathrm{~Hz}, 2 \times \mathrm{H}^{B n}\right), 5.43\left(\mathrm{~d}, 2 \mathrm{H},{ }^{3} J_{1,2}=3.7 \mathrm{~Hz}, \mathrm{H} 1[\mathrm{~B}, \mathrm{E}]\right), 6.83-7.21\left(\mathrm{~m}, 70 \mathrm{H}, 70 \times \mathrm{HAr}^{B n}\right), 7.33(\mathrm{~d}, 2 \mathrm{H}$, $\left.{ }^{3} \mathrm{~J}=8.6 \mathrm{~Hz}, \mathrm{H}_{o}{ }^{\mathrm{CD}}[\mathrm{A}, \mathrm{D}]\right), 7.75\left(\mathrm{t}, 2 \mathrm{H},{ }^{3} \mathrm{~J}=7.5 \mathrm{~Hz}, \mathrm{H}_{m}{ }^{\mathrm{H}}[\mathrm{A}, \mathrm{D}]\right), 7.97\left(\mathrm{t}_{\mathrm{b}}, 2 \mathrm{H},{ }^{3} \mathrm{~J}=8.1 \mathrm{~Hz}, \mathrm{H}_{m}{ }^{\mathrm{CD}}[\mathrm{A}, \mathrm{D}]\right), 8.94\left(\mathrm{~d}_{\mathrm{b}}\right.$, $\left.2 \mathrm{H},{ }^{3} \mathrm{~J}=7.2 \mathrm{~Hz}, \mathrm{H}_{o}{ }^{\mathrm{H}}[\mathrm{A}, \mathrm{D}]\right), 9.11\left(\mathrm{~d}, 2 \mathrm{H},{ }^{3} \mathrm{~J}=4.7 \mathrm{~Hz}, \pi[1,4]\right), 9.14\left(\mathrm{~d}, 2 \mathrm{H},{ }^{3} J=4.7 \mathrm{~Hz}, \pi^{\mathrm{Pf}}[3,6]\right), 9.43(\mathrm{~d}, 2 \mathrm{H}$, $\left.{ }^{3} J=4.6 \mathrm{~Hz}, \pi[1,4]\right), 9.67\left(\mathrm{~d}, 2 \mathrm{H},{ }^{3} \mathrm{~J}=4.7 \mathrm{~Hz}, \pi^{\mathrm{Ph}}[3,6]\right) .{ }^{13} \mathrm{C}$ from 2D HSQC $\delta: 64.4(\mathrm{C} 6[\mathrm{C}, \mathrm{F}]), 66.5$ $\left(\mathrm{CH}_{2}{ }^{\mathrm{H}}[\mathrm{A}, \mathrm{D}]\right), 68.5$ (C6[B,E]), $68.67(\mathrm{C} 6[\mathrm{~A}, \mathrm{D}]), 69.73\left(\mathrm{CH}_{2}{ }^{\mathrm{CD}}[\mathrm{A}, \mathrm{D}]\right), 70.4(\mathrm{C} 5[\mathrm{~B}, \mathrm{E}]), 71.0\left(\mathrm{CH}_{2}{ }^{\mathrm{Bn}-6}[\mathrm{C}, \mathrm{F}]\right), 71.2$ (C5[C,F]), $72.3(\mathrm{C} 4[\mathrm{C}, \mathrm{F}]), 71.4(\mathrm{C} 5[\mathrm{~A}, \mathrm{D}]), 71.8\left(2 \times \mathrm{CH}_{2}{ }^{\mathrm{Bn}}\right), 72.6\left(2 \times \mathrm{CH}_{2}{ }^{\mathrm{Bn}}\right), 72.8\left(\mathrm{CH}_{2}{ }^{\mathrm{Bn}-6}[\mathrm{~B}, \mathrm{E}]\right), 73.3$ $\left(2 \times \mathrm{CH}_{2}{ }^{\mathrm{Bn}}\right), 74.0\left(2 \times \mathrm{CH}_{2}{ }^{\mathrm{Bn}}\right), 75.9\left(2 \times \mathrm{CH}_{2}{ }^{\mathrm{Bn}}\right), 76.4\left(2 \times \mathrm{CH}_{2}{ }^{\mathrm{Bn}}\right), 77.4(\mathrm{C} 2[\mathrm{~B}, \mathrm{E}]), 79.2(\mathrm{C} 2[\mathrm{~A}, \mathrm{D}]), 79.8(\mathrm{C} 4[\mathrm{~B}, \mathrm{E}])$, 80.2 (C2[C,F]), 80.3 (C4[A,D]), 80.5 (C3[A,D]), 81.0 (C3[B,E]), 81.1 (C3[C,F]), 95.9 (C1[A,D]), 97.1 (C1[B,E]), $97.8(C 1[C, F]), 111.1\left(C_{o}{ }^{C D}[A, D]\right), 120.1\left(C_{m}{ }^{H}[A, D]\right), 121.8(C \pi[2,5]), 122.3\left(C \pi^{\prime}[2,5]\right), 124.1$ $\left(C_{p}{ }^{B n-6}[C, F]\right), 125.49\left(2 \times C_{m}^{B n-6}[C, F]\right), 125.53\left(2 \times C_{o}^{B n-6}[C, F]\right), 126.4-128.6\left(70 \times C A r^{B n}\right), 129.5\left(C \pi^{P f}[3,6]\right)$, $130.9\left(C_{m}{ }^{C D}[A, D]\right), 132.9(C \pi[1,4]), 135.5(C \pi[1,4]), 135.6\left(C \pi^{\mathrm{Ph}}[3,6]\right), 137.2\left(\mathrm{Co}_{o}{ }^{\mathrm{H}}[\mathrm{A}, \mathrm{D}]\right) .{ }^{19} \mathrm{~F}$ NMR $(376$ $\left.\mathrm{MHz}, \mathrm{CDCl}_{3}, 298 \mathrm{~K}\right): \delta-135.78\left(\mathrm{~d}, 2 \mathrm{~F}, J=22.9 \mathrm{~Hz}, \mathrm{~F}_{\text {ortho }}\right),-136.33\left(\mathrm{dt}, 2 \mathrm{~F}, J=7.5,25.1 \mathrm{~Hz}, \mathrm{~F}_{\text {ortho }}\right),-136.76$ (dt, $\left.2 \mathrm{~F}, J=6.3,23.9 \mathrm{~Hz}, \mathrm{~F}_{\text {ortho }}\right),-139.76\left(\mathrm{~d}, 2 \mathrm{~F}, J=24.3 \mathrm{~Hz}, \mathrm{~F}_{\text {ortho }}\right),-150.50\left(\mathrm{t}, 2 \mathrm{~F}, J=20.8 \mathrm{~Hz}, \mathrm{~F}_{\text {para }}\right),-154.20$ $\left(\mathrm{t}, 2 \mathrm{~F}, \mathrm{~J}=21.0 \mathrm{~Hz}, \mathrm{~F}_{\text {para }}\right),-160.57\left(\mathrm{~m}, 4 \mathrm{~F}, \mathrm{~F}_{\text {meta }}\right),-163.36\left(\mathrm{dt}, 2 \mathrm{~F}, J=7.0,22.5 \mathrm{~Hz}, \mathrm{~F}_{\text {meta }}\right),-163.66(\mathrm{dt}, 2 \mathrm{~F}, J$ $=7.5,23.8 \mathrm{~Hz}, \mathrm{~F}_{\text {meta }}$ ). HRMS (ESI-TOF, $\mathrm{m} / \mathrm{z}$ ) for $\mathrm{C}_{218} \mathrm{H}_{185} \mathrm{~N}_{6} \mathrm{O}_{32} \mathrm{~F}_{20}[\mathrm{M}+\mathrm{H}]^{+}$, calculated 3778.27086, found 3778.2676. UV-vis $\left(\mathrm{CH}_{2} \mathrm{Cl}_{2}\right): \lambda\left(\varepsilon, \mathrm{M}^{-1} . \mathrm{cm}^{-1}\right)=571\left(1.94 \times 10^{5}\right), 595\left(1.29 \times 10^{5}\right), 724\left(2.29 \times 10^{4}\right), 786$ $\left(8.34 \times 10^{3}\right), 907\left(4.28 \times 10^{3}\right), 1038\left(1.33 \times 10^{4}\right) \mathrm{nm}$. 


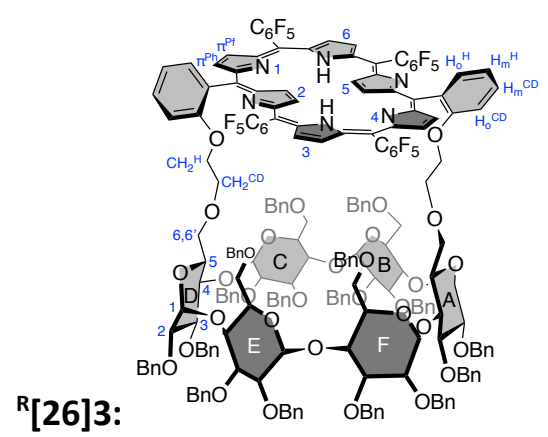

${ }^{1} \mathrm{H}$ NMR $\left(500 \mathrm{MHz}, \mathrm{CDCl}_{3}, 283 \mathrm{~K}\right): \delta-3.04--2.94\left(\mathrm{~m}, 4 \mathrm{H}, \pi[2,5], \pi^{\prime}[2,5]\right),-2.52\left(\mathrm{~s}_{\mathrm{b}}, 2 \mathrm{H}, 2 \times \mathrm{NH}\right), 1.86(\mathrm{~d}$, $\left.2 \mathrm{H},{ }^{2} J_{6,6^{\prime}}=11.3 \mathrm{~Hz}, \mathrm{H} 6[\mathrm{C}, \mathrm{F}]\right), 1.92\left(\mathrm{~d}, 2 \mathrm{H},{ }^{2} \mathrm{~J}=12.0 \mathrm{~Hz}, \mathrm{CHH}^{\mathrm{Bn}-6}[\mathrm{C}, \mathrm{F}]\right), 2.10\left(\mathrm{br}, 2 \mathrm{H}, \mathrm{CH} \mathrm{H}^{\mathrm{CD}}[\mathrm{A}, \mathrm{D}]\right), 2.16(\mathrm{~d}$, $\left.2 \mathrm{H},{ }^{2} \mathrm{~J}=12.0 \mathrm{~Hz}, \mathrm{CH}{ }^{\mathrm{Bn}-6}[\mathrm{C}, \mathrm{F}]\right), 2.22\left(\mathrm{~d}, 2 \mathrm{H},{ }^{2} \mathrm{~J}_{6,6^{\prime}}=11.0 \mathrm{~Hz}, \mathrm{H} 6[\mathrm{~A}, \mathrm{D}]\right), 2.36\left(\mathrm{br}, 2 \mathrm{H}, \mathrm{H} 6^{\prime}[\mathrm{C}, \mathrm{F}]\right), 2.47(\mathrm{br}, 2 \mathrm{H}$, $\left.\mathrm{CH} H^{\mathrm{CD}}[\mathrm{A}, \mathrm{D}]\right), 2.51\left(\mathrm{~d}, 2 \mathrm{H},{ }^{2} \mathrm{~J}_{6,6^{\prime}}=11.6 \mathrm{~Hz}, \mathrm{H} 6[\mathrm{~B}, \mathrm{E}]\right), 2.74\left(\mathrm{br}, 2 \mathrm{H}, \mathrm{H} 6^{\prime}[\mathrm{A}, \mathrm{D}]\right), 2.88-3.01(\mathrm{~m}, 6 \mathrm{H}, \mathrm{H} 2[\mathrm{C}, \mathrm{F}]$, $\mathrm{H} 5[\mathrm{~A}, \mathrm{D}], \mathrm{H} 5[\mathrm{~B}, \mathrm{E}]), 3.06(\mathrm{br}, 2 \mathrm{H}, \mathrm{H} 5[\mathrm{C}, \mathrm{F}]), 3.15$ (dd, $\left.2 \mathrm{H},{ }^{3} \mathrm{~J}_{1,2}=3.0,{ }^{3} \mathrm{~J}_{2,3}=9.8 \mathrm{~Hz}, \mathrm{H} 2[\mathrm{~A}, \mathrm{D}]\right), 3.25$ (dd, $2 \mathrm{H}$, $\left.{ }^{3} J_{1,2}=3.6,{ }^{3} J_{2,3}=9.8 \mathrm{~Hz}, \mathrm{H} 2[\mathrm{~B}, \mathrm{E}]\right), 3.27-3.36\left(\mathrm{~m}, 6 \mathrm{H}, \mathrm{H} 4[\mathrm{~A}, \mathrm{D}], \mathrm{H} 4[\mathrm{C}, \mathrm{F}], \mathrm{H} 6^{\prime}[\mathrm{B}, \mathrm{E}]\right), 3.58\left(\mathrm{t}, 2 \mathrm{H},{ }^{3} \mathrm{~J}_{3,3}=33_{3,4}=\right.$ $9.2 \mathrm{~Hz}, \mathrm{H} 3[\mathrm{~A}, \mathrm{D}]), \quad 3.63\left(\mathrm{t}, 2 \mathrm{H}, 3_{3,3}^{3}=3_{3,4}=9.2 \mathrm{~Hz}, \mathrm{H} 3[\mathrm{C}, \mathrm{F}]\right), 3.73-3.86(\mathrm{~m}, 8 \mathrm{H}, \mathrm{H} 3[\mathrm{~B}, \mathrm{E}], \mathrm{H} 4[\mathrm{~B}, \mathrm{E}]$, $\left.\mathrm{CH}_{2}{ }^{\mathrm{H}}[\mathrm{A}, \mathrm{D}]\right), 3.93\left(\mathrm{~d}, 2 \mathrm{H},{ }^{2} \mathrm{~J}=12.1 \mathrm{~Hz}, \mathrm{CHH}^{\mathrm{Bn}-6}[\mathrm{~B}, \mathrm{E}]\right), 4.00-4.12\left(\mathrm{~m}, 8 \mathrm{H}, \mathrm{H} 1[\mathrm{C}, \mathrm{F}], \mathrm{CH} H^{\mathrm{Bn}-6}[\mathrm{~B}, \mathrm{E}], 4 \times \mathrm{H}^{\mathrm{Bn}}\right), 4.16$ $\left(\mathrm{br}, 2 \mathrm{H}, \mathrm{H}_{p}{ }^{\mathrm{Bn}-6}[\mathrm{C}, \mathrm{F}]\right), 4.23\left(\mathrm{~d}, 2 \mathrm{H},{ }^{2} \mathrm{~J}=12.2 \mathrm{~Hz}, 2 \times \mathrm{H}^{\mathrm{Bn}}\right), 4.25\left(\mathrm{~d}, 2 \mathrm{H},{ }^{2} \mathrm{~J}=12.4 \mathrm{~Hz}, 2 \times \mathrm{H}^{\mathrm{Bn}}\right), 4.32\left(\mathrm{~d}, 2 \mathrm{H},{ }^{2} \mathrm{~J}=\right.$ 12.4 Hz, 2xH $\left.{ }^{B n}\right), 4.44-4.65\left(m, 16 \mathrm{H}, \mathrm{H} 1[\mathrm{~A}, \mathrm{D}], 2 \times \mathrm{H}_{m}{ }^{B n-6}[\mathrm{C}, \mathrm{F}], 10 \times \mathrm{H}^{\mathrm{Bn}}\right), 4.72\left(\mathrm{~d}, 4 \mathrm{H}, 3^{3} \mathrm{~J}=7.1 \mathrm{~Hz}, 2 \times \mathrm{H}_{0}^{\mathrm{Bn}-}\right.$ $\left.{ }^{6}[C, F]\right), 4.85\left(d, 2 \mathrm{H},{ }^{2} J=11.0 \mathrm{~Hz}, 2 \times \mathrm{H}^{B n}\right), 5.19\left(\mathrm{~d}, 2 \mathrm{H},{ }^{2} J=10.8 \mathrm{~Hz}, 2 \times \mathrm{H}^{\mathrm{Bn}}\right), 5.21\left(\mathrm{~d}, 2 \mathrm{H},{ }^{3} \mathrm{~J}_{1,2}=3.6 \mathrm{~Hz}\right.$, $\mathrm{H} 1[\mathrm{~B}, \mathrm{E}]), 6.89-7.20\left(\mathrm{~m}, 70 \mathrm{H}, 70 \times \mathrm{HAr}^{\mathrm{Bn}}\right), 7.28\left(\mathrm{~d}_{\mathrm{b}}, 2 \mathrm{H},{ }^{3} \mathrm{~J}=8.6 \mathrm{~Hz}, \mathrm{H}_{0}{ }^{\mathrm{CD}}[\mathrm{A}, \mathrm{D}]\right), 7.71\left(\mathrm{t}, 2 \mathrm{H},{ }^{3} \mathrm{~J}=7.5 \mathrm{~Hz}\right.$, $\left.\mathrm{H}_{m}{ }^{\mathrm{H}}[\mathrm{A}, \mathrm{D}]\right), 7.90\left(\mathrm{t}, 2 \mathrm{H},{ }^{3} \mathrm{~J}=8.0 \mathrm{~Hz}, \mathrm{H}_{m}{ }^{\mathrm{CD}}[\mathrm{A}, \mathrm{D}]\right), 8.95\left(\mathrm{~d}, 2 \mathrm{H},{ }^{3} \mathrm{~J}=7.2 \mathrm{~Hz}, \mathrm{H}_{o}{ }^{H}[\mathrm{~A}, \mathrm{D}]\right), 9.01\left(\mathrm{~d}, 2 \mathrm{H},{ }^{3} \mathrm{~J}=4.8 \mathrm{~Hz}\right.$, $\pi[3,6]), 9.03\left(\mathrm{~d}, 2 \mathrm{H},{ }^{3} J=4.6 \mathrm{~Hz}, \pi^{\mathrm{Pf}}[1,4]\right), 9.30\left(\mathrm{~d}, 2 \mathrm{H},{ }^{3} \mathrm{~J}=4.7 \mathrm{~Hz}, \pi^{\prime}[3,6]\right), 9.51\left(\mathrm{~d}, 2 \mathrm{H},{ }^{3} J=4.8 \mathrm{~Hz}, \pi^{\mathrm{Ph}}[1,4]\right)$. ${ }^{13} \mathrm{C}$ from 2D HSQC $\delta: 66.2\left(\mathrm{CH}_{2}{ }^{\mathrm{H}}[\mathrm{A}, \mathrm{D}]\right), 67.0(\mathrm{C} 6[\mathrm{C}, \mathrm{F}]), 68.3\left(\mathrm{CH}_{2}{ }^{\mathrm{CD}}[\mathrm{A}, \mathrm{D}]\right), 68.5(\mathrm{C} 6[\mathrm{~B}, \mathrm{E}]), 69.1$ (C6[A,D]), 70.3 (C5[C,F]), 70.5 (C5[A,D]), 70.8 (C5[B,E]), $71.0\left(\mathrm{CH}_{2}{ }^{\mathrm{Bn}-6}[\mathrm{C}, \mathrm{F}]\right), 71.8-73.4\left(\mathrm{CH}_{2}{ }^{\mathrm{Bn}-6}[\mathrm{~B}, \mathrm{E}], 6 \times \mathrm{CH}_{2}{ }^{\mathrm{Bn}}\right), 74.0$ $\left(2 \times \mathrm{CH}_{2}{ }^{\mathrm{Bn}}\right), 75.2(\mathrm{C} 4[\mathrm{C}, \mathrm{F}]), 75.9\left(2 \times \mathrm{CH}_{2}{ }^{\mathrm{Bn}}\right), 76.0\left(2 \times \mathrm{CH}_{2}{ }^{\mathrm{Bn}}\right), 77.7$ (C2[B,E]), $78.6(\mathrm{C} 2[\mathrm{~A}, \mathrm{D}]), 79.5(\mathrm{C} 2[\mathrm{C}, \mathrm{F}])$, 80.3 (C3[A,D]), 80.4 (C4[A,D], C4[B,E]), 80.6 (C3[B,E], C3[C,F]), 97.5 (C1[A,D]), 98.2 (C1[B,E]), 98.4 (C1[C,F]), $111.9\left(\mathrm{C}_{o}^{\mathrm{CD}}[\mathrm{A}, \mathrm{D}]\right), 119.9\left(\mathrm{C}_{m}{ }^{\mathrm{H}}[\mathrm{A}, \mathrm{D}]\right), 122.5\left(\mathrm{C} \pi[2,5], \mathrm{C}^{\prime}[2,5]\right), 124.8\left(\mathrm{C}_{p}{ }^{\mathrm{Bn}-6}[\mathrm{C}, \mathrm{F}]\right), 125.3\left(2 \times \mathrm{C}_{o}{ }^{\mathrm{Bn}-}\right.$ $\left.{ }^{6}[C, F]\right), 125.6\left(2 \times C_{m}^{B n-6}[C, F]\right), 125.8-129.2\left(70 \times C A r^{B n}\right), 129.7\left(C \pi^{P f}[1,4]\right), 130.9\left(C_{m}^{C D}[A, D]\right), 132.7$ $(C \pi[3,6]), 135.0\left(C \pi^{\prime}[3,6]\right), 135.6\left(C \pi^{\mathrm{Ph}}[1,4]\right), 136.2\left(\mathrm{C}_{o}{ }^{\mathrm{H}}[\mathrm{A}, \mathrm{D}]\right) .{ }^{19} \mathrm{~F}$ NMR $\left(376 \mathrm{MHz}, \mathrm{CDCl}_{3}, 298 \mathrm{~K}\right): \delta-$ 136.14 (d, 2F, J = 24.0 Hz, Fortho), -136.33 (d, 2F, J = 24.1 Hz, Fortho), -136.71 (dt, 2F, J = 5.7, $24.1 \mathrm{~Hz}, \mathrm{~F}_{\text {ortho }}$ ), $-139.23\left(\mathrm{~d}, 2 \mathrm{~F}, J=24.8 \mathrm{~Hz}, \mathrm{~F}_{\text {ortho }}\right),-150.64\left(\mathrm{t}, 2 \mathrm{~F}, J=20.8 \mathrm{~Hz}, \mathrm{~F}_{\text {para }}\right),-153.90\left(\mathrm{t}, 2 \mathrm{~F}, J=21.2 \mathrm{~Hz}, \mathrm{~F}_{\text {para }}\right)$, $160.66\left(\mathrm{~m}, 2 \mathrm{~F}, \mathrm{~F}_{\text {meta }}\right),-160.72\left(\mathrm{~m}, 2 \mathrm{~F}, \mathrm{~F}_{\text {meta }}\right),-162.40\left(\mathrm{dt}, 2 \mathrm{~F}, J=7.1,23.9 \mathrm{~Hz}, \mathrm{~F}_{\text {meta }}\right),-163.52(\mathrm{t}, 2 \mathrm{~F}, \mathrm{~J}=$ $22.3 \mathrm{~Hz}, \mathrm{~F}_{\text {meta }}$ ). HRMS (ESI-TOF, $\left.m / z\right)$ for $\mathrm{C}_{218} \mathrm{H}_{185} \mathrm{~N}_{6} \mathrm{O}_{32} \mathrm{~F}_{20}[\mathrm{M}+\mathrm{H}]^{+}$, calculated 3778.27086, found 3778.2663. UV-vis $\left(\mathrm{CH}_{2} \mathrm{Cl}_{2}\right): \lambda\left(\varepsilon, \mathrm{M}^{-1} . \mathrm{cm}^{-1}\right)=573\left(1.47 \times 10^{5}\right), 722\left(1.58 \times 10^{4}\right), 784\left(7.30 \times 10^{3}\right), 906$ $\left(3.64 \times 10^{3}\right), 1035\left(8.22 \times 10^{3}\right) \mathrm{nm}$. 


\section{$3.4{ }^{\mathrm{M}}[\mathbf{2 8}] 2$ by reduction of ${ }^{\mathrm{R}}[\mathbf{2 6}] 2$}

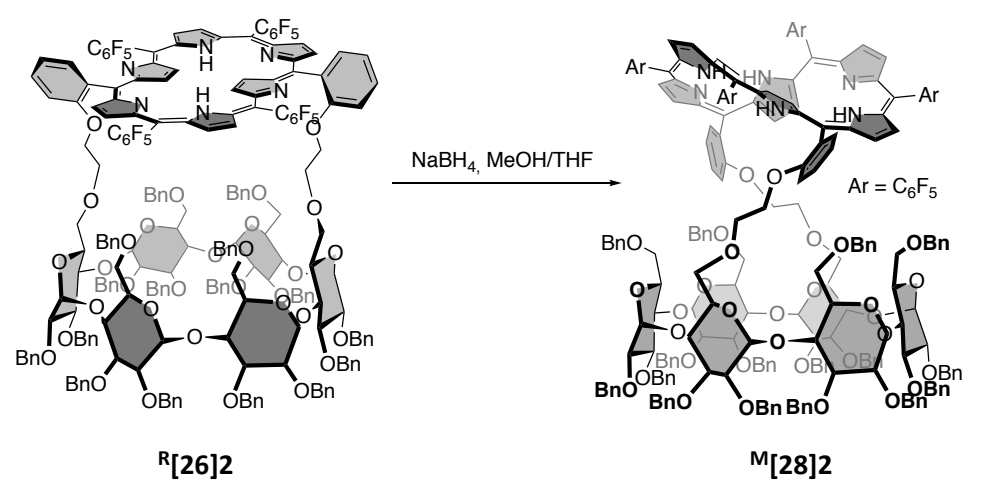

Under inert atmosphere, $3.5 \mathrm{mg}(90 \mu \mathrm{mol})$ of $\mathrm{NaBH}_{4}$ and $300 \mu \mathrm{L}$ of $\mathrm{MeOH}$ were successively added to a solution of $17 \mathrm{mg}(4.5 \mu \mathrm{mol})$ of ${ }^{\mathrm{R}}[\mathbf{2 6}] \mathbf{2}$ in $3.2 \mathrm{~mL}$ of THF. The mixture was stirred at room temperature for 40 minutes. The solvent was then concentrated under reduced pressure (without heating) and the residue was dissolved in $6 \mathrm{~mL}$ of DCM. The organic layer was vigorously washed with $\mathrm{H}_{2} \mathrm{O}(3 \times 10 \mathrm{~mL})$, then concentrated under reduced pressure (without heating) to afford compound ${ }^{\mathrm{M}}[\mathbf{2 8}] \mathbf{2}$ with 90:10 d.r. as a blue solid (quantitative yield).

${ }^{1}$ H NMR: see above, ${ }^{\mathrm{M}}[\mathbf{2 8}] \mathbf{2} /{ }^{\mathrm{M}}[\mathbf{2 8}] 3$ equilibrated mixture $\left(\mathrm{CDCl}_{3}, 330 \mathrm{~K}\right)$

\section{$3.5{ }^{\mathrm{M}}[28] 3$ by reduction of ${ }^{\mathrm{R}}[26] 3$}

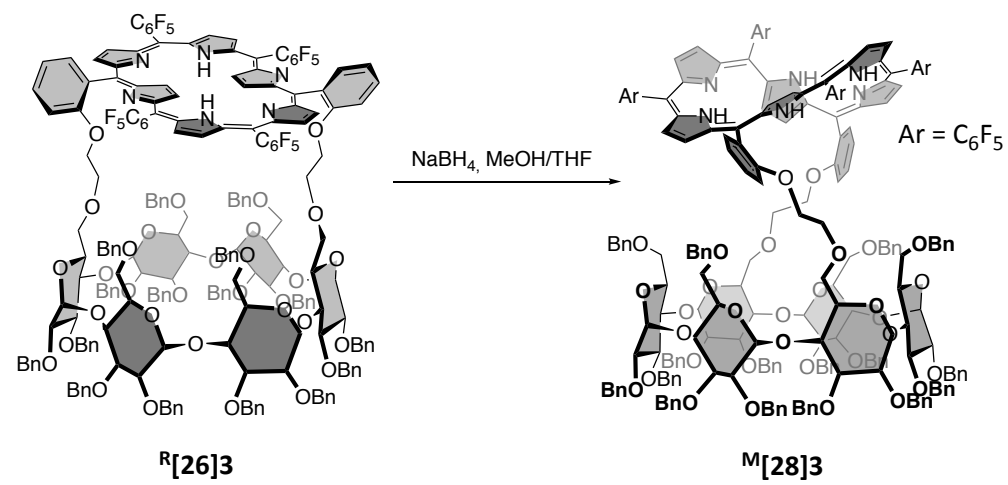

Under inert atmosphere, $10.5 \mathrm{mg}(270 \mu \mathrm{mol})$ of $\mathrm{NaBH}_{4}$ and $300 \mu \mathrm{L}$ of $\mathrm{MeOH}$ were successively added to a solution of $17 \mathrm{mg}(4.5 \mu \mathrm{mol})$ of ${ }^{\mathrm{R}}[26] 3$ in $3.3 \mathrm{~mL}$ of THF. The mixture was stirred at room temperature for 40 minutes. The solvent was then concentrated under reduced pressure (without heating) and the residue was dissolved in $6 \mathrm{~mL}$ of DCM. The organic layer was vigorously washed with $\mathrm{H}_{2} \mathrm{O}(3 \times 10 \mathrm{~mL})$, then concentrated under reduced pressure (without heating) to afford compound $(P)-$ M[28]3 with 15:85 d.r. as a blue solid (quantitative yield).

${ }^{1}$ H NMR: see above, ${ }^{\mathrm{M}}[\mathbf{2 8}] \mathbf{2} /{ }^{\mathrm{M}}[\mathbf{2 8}] \mathbf{3}$ equilibrated mixture $\left(\mathrm{CDCl}_{3}, 330 \mathrm{~K}\right)$ 
4 Thermodynamic equilibration processes

$4.1{ }^{1} \mathrm{H}$ NMR (a-c) and UV-vis (d) evolution of ${ }^{\mathrm{R}}[28] 1$ to ${ }^{\mathrm{M}}[28] 2 / \mathrm{M}[28] 3$

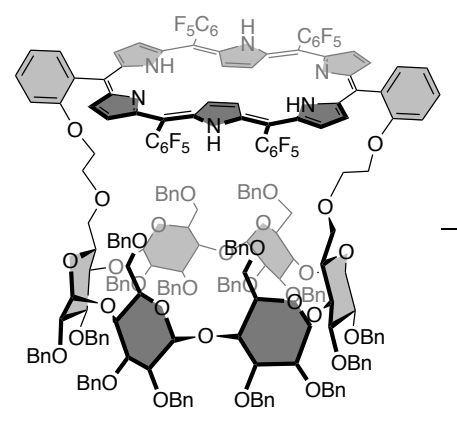

${ }^{\mathrm{R}}[28] 1$

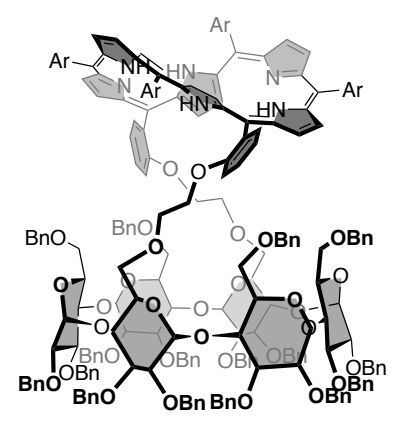

M[28]2

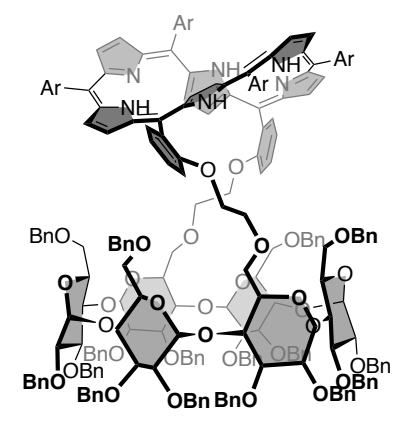

$\mathrm{Ar}=\mathrm{C}_{6} \mathrm{~F}_{5}$

${ }^{1} \mathrm{H}$ NMR, THF- $d_{8}, 298 \mathrm{~K}$

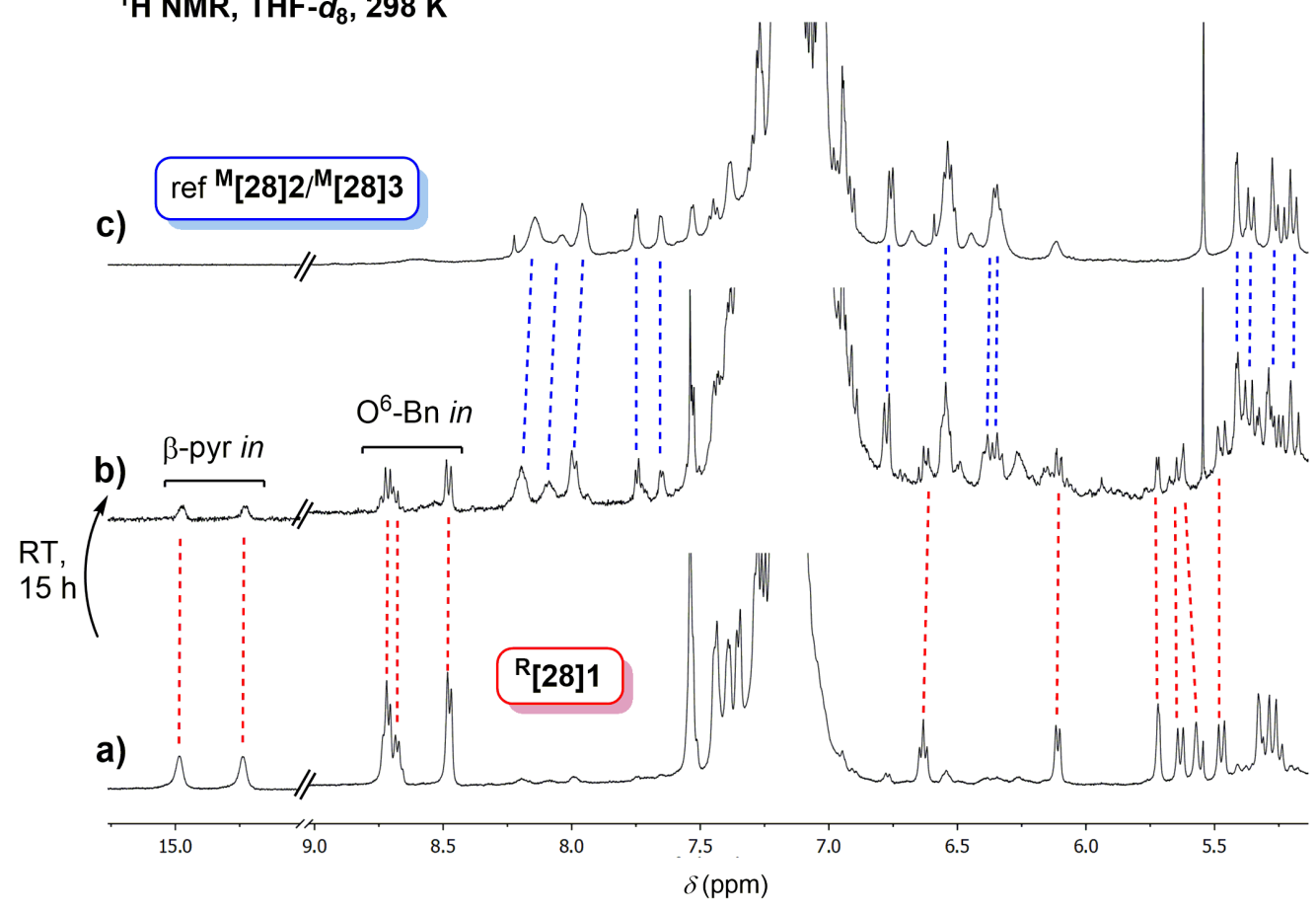

d) UV-vis spectra, THF, RT

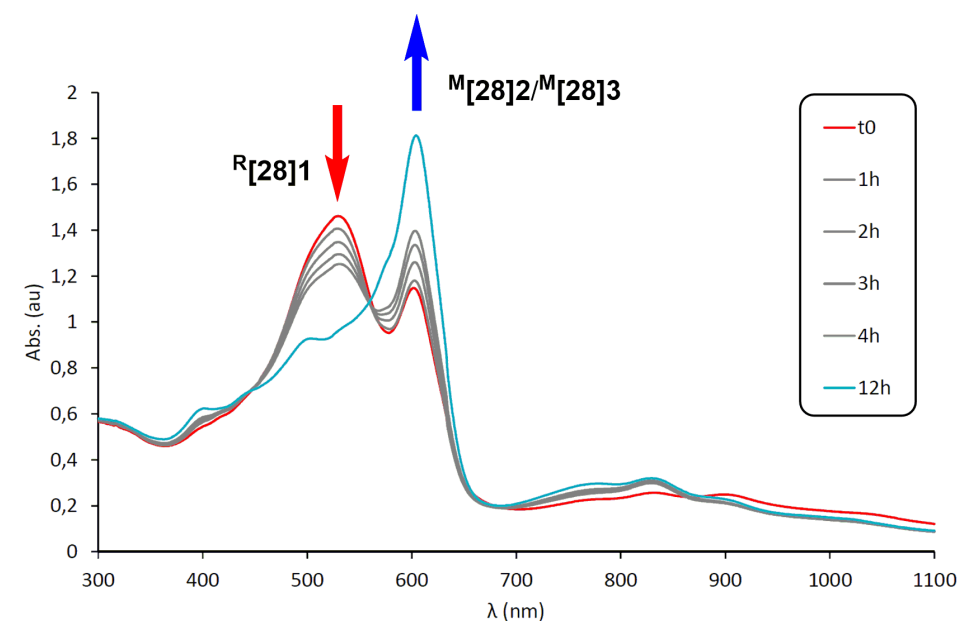


4.2 ${ }^{1} \mathrm{H}$ and ${ }^{19} \mathrm{~F}$ NMR evolution of ${ }^{\mathrm{M}}[28] 2$ (a) and ${ }^{\mathrm{M}}[28] 3$ (b) to ${ }^{\mathrm{M}}[\mathbf{2 8}] 2 /{ }^{\mathrm{M}}[\mathbf{2 8}] 3$ (spectra recorded in $\mathrm{CDCl}_{3}$ at $298 \mathrm{~K}$ )
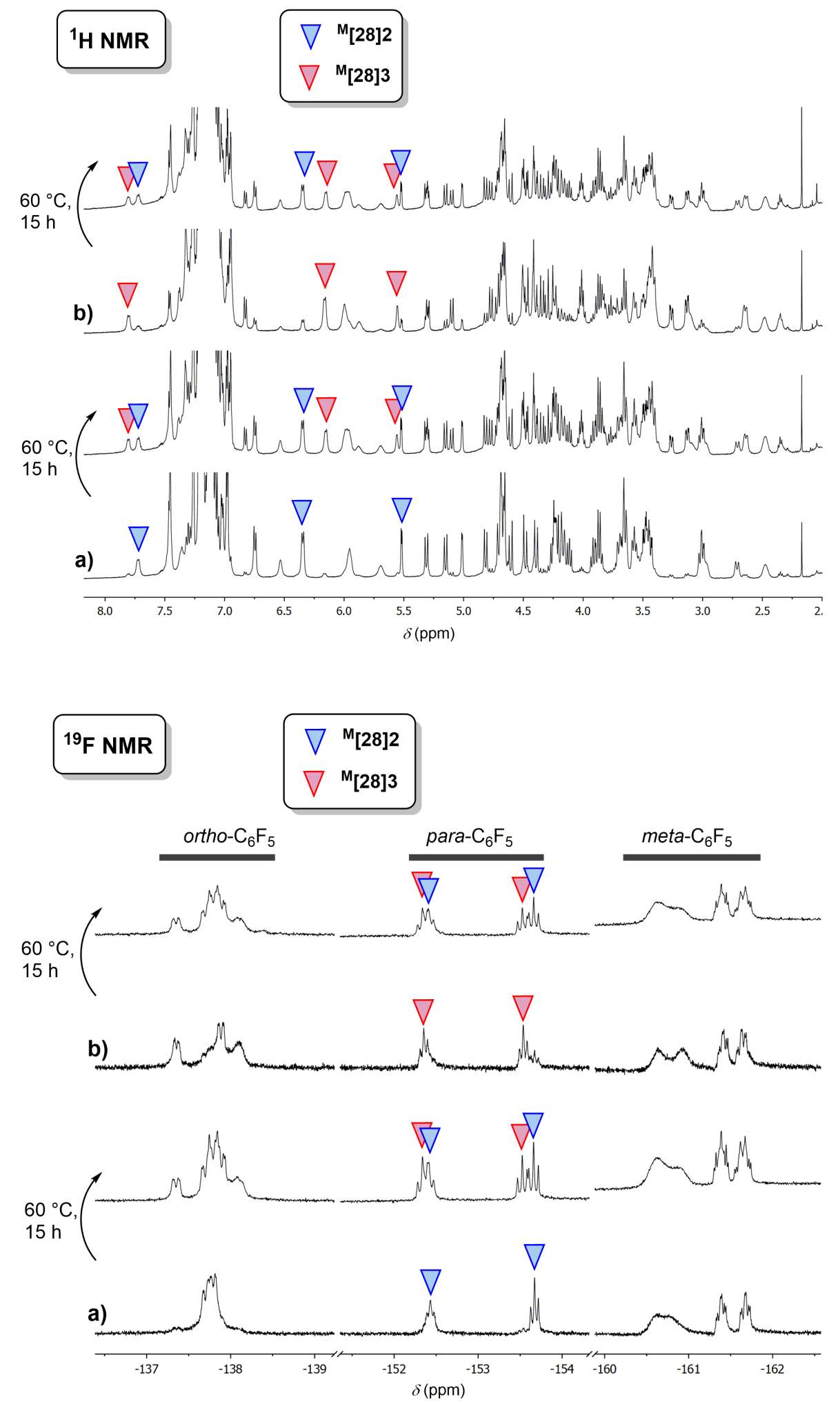


\section{3 ${ }^{1} \mathrm{H}$ NMR evolution of ${ }^{\mathrm{R}}[26] 2$ to ${ }^{\mathrm{R}}[26] 1$}

\subsection{1 ${ }^{1} \mathrm{H} N M R$ spectra of the conformational isomerization $\left(\mathrm{CDCl}_{3}, 330 \mathrm{~K}\right)$}

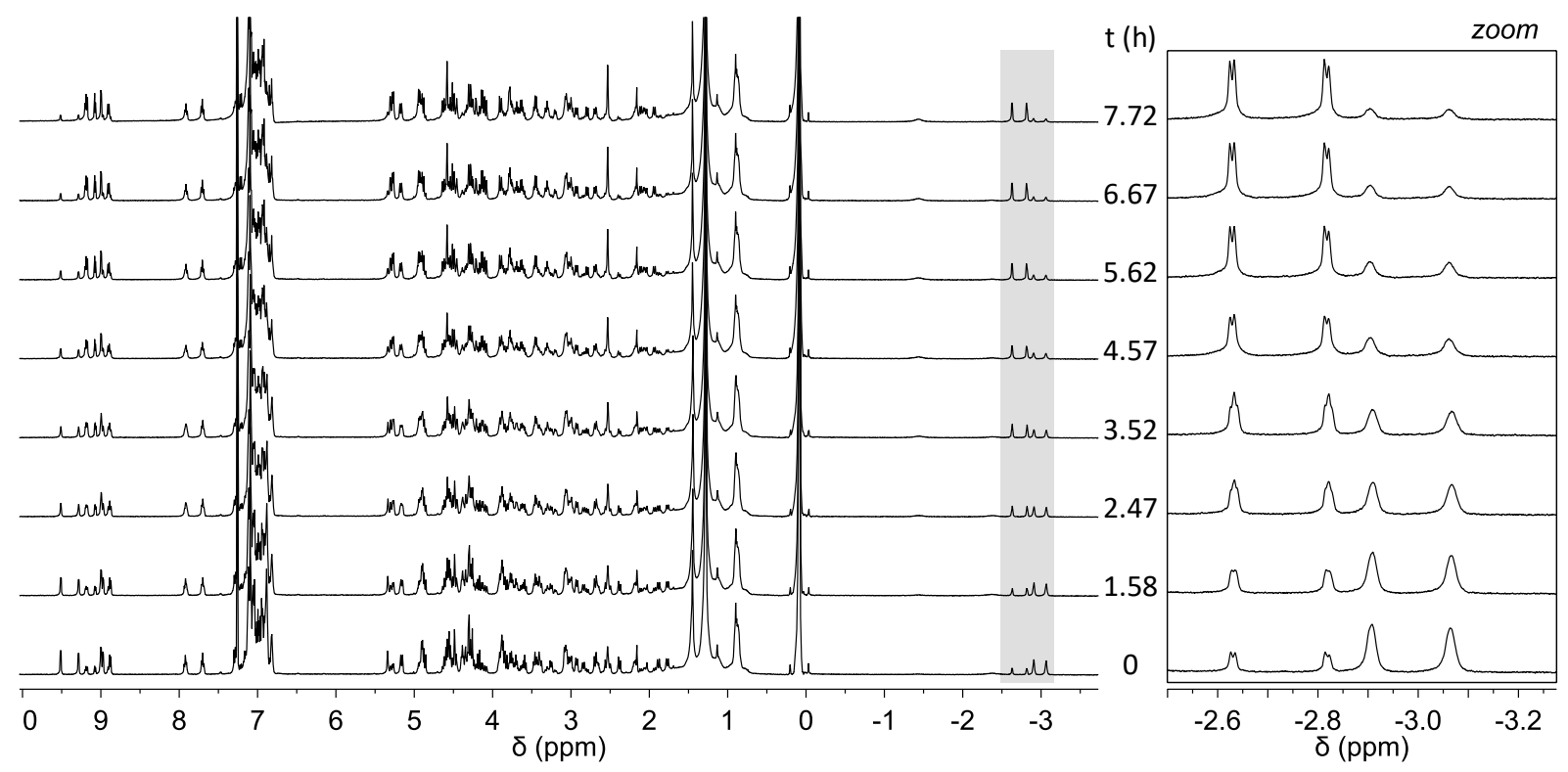

\subsubsection{Kinetic analysis of the conformational isomerization}

In this case, the non-equilibrated model fitted better to the collected data. We thus considered the following process.

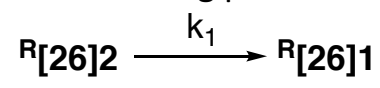

Rate $=d\left[{ }^{R}[26] 1\right] / d t=-d\left[{ }^{R}[26] 2\right] / d t=k_{1}\left[{ }^{R}[26] 2\right]$

Analysis of the kinetic profiles with Dynafit software gave the kinetic constant $\mathrm{k}_{1}=0.21 \mathrm{~h}^{-1}$ from ${ }^{1} \mathrm{H}$ NMR data at $330 \mathrm{~K}$ in $\mathrm{CDCl}_{3}$ (average intensities for signals at -2.63 and -2.82 ppm for ${ }^{\mathrm{R}}[\mathbf{2 6}] 1$ and signals at -2.91 and -3.06 ppm for ${ }^{\mathrm{R}}[\mathbf{2 6}] \mathbf{2}$, see Part 4.3.1).

Eyring's equation was used to calculate free energy of activation $\left(\Delta G^{\ddagger}\right)$ of the isomerization process:

$k=\frac{k_{B} T}{h} e^{\left(\frac{-\Delta G^{\ddagger}}{R T}\right)}$

$\mathrm{k}_{\mathrm{B}}$ is Boltzmann's constant $\left(\mathrm{k}_{\mathrm{B}}=1.3806 \times 10^{-23} \mathrm{~J} . \mathrm{K}^{-1}\right)$

$h$ is Planck's constant $\left(h=6.626 \times 10^{-34} \mathrm{~J} . \mathrm{s}\right)$

$R$ is the gaz constant $\left(R=8.314 \mathrm{~J} \cdot \mathrm{K}^{-1} \cdot \mathrm{mol}^{-1}\right)$

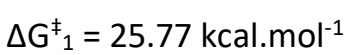

Isomerization in $\mathrm{CDCl}_{3}(330 \mathrm{~K})$

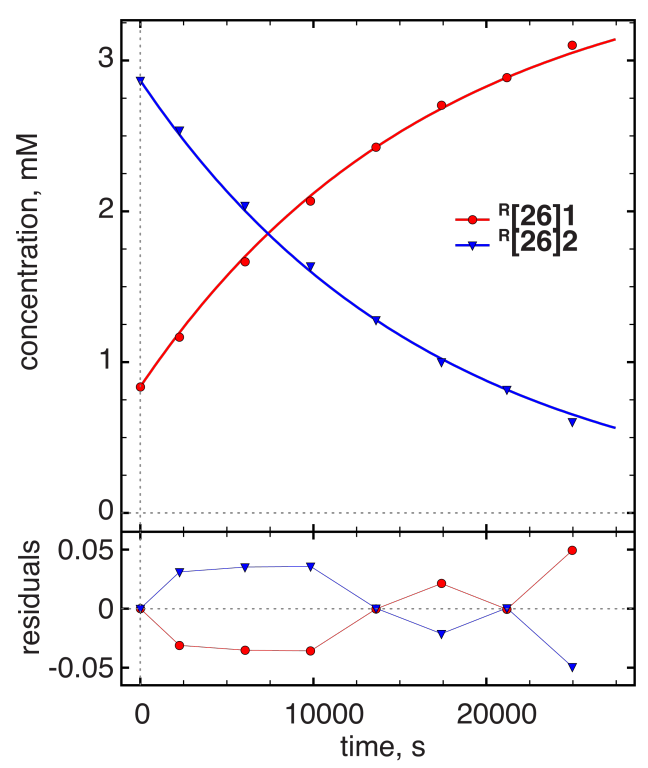




\section{4 ${ }^{1} \mathrm{H}$ NMR evolution of ${ }^{\mathrm{R}}[26] 3$ to ${ }^{\mathrm{R}}[26] 1$}

\subsection{1 ${ }^{1} \mathrm{H} N M R$ spectra of the conformational isomerization $\left(\mathrm{CDCl}_{3}, 330 \mathrm{~K}\right)$}
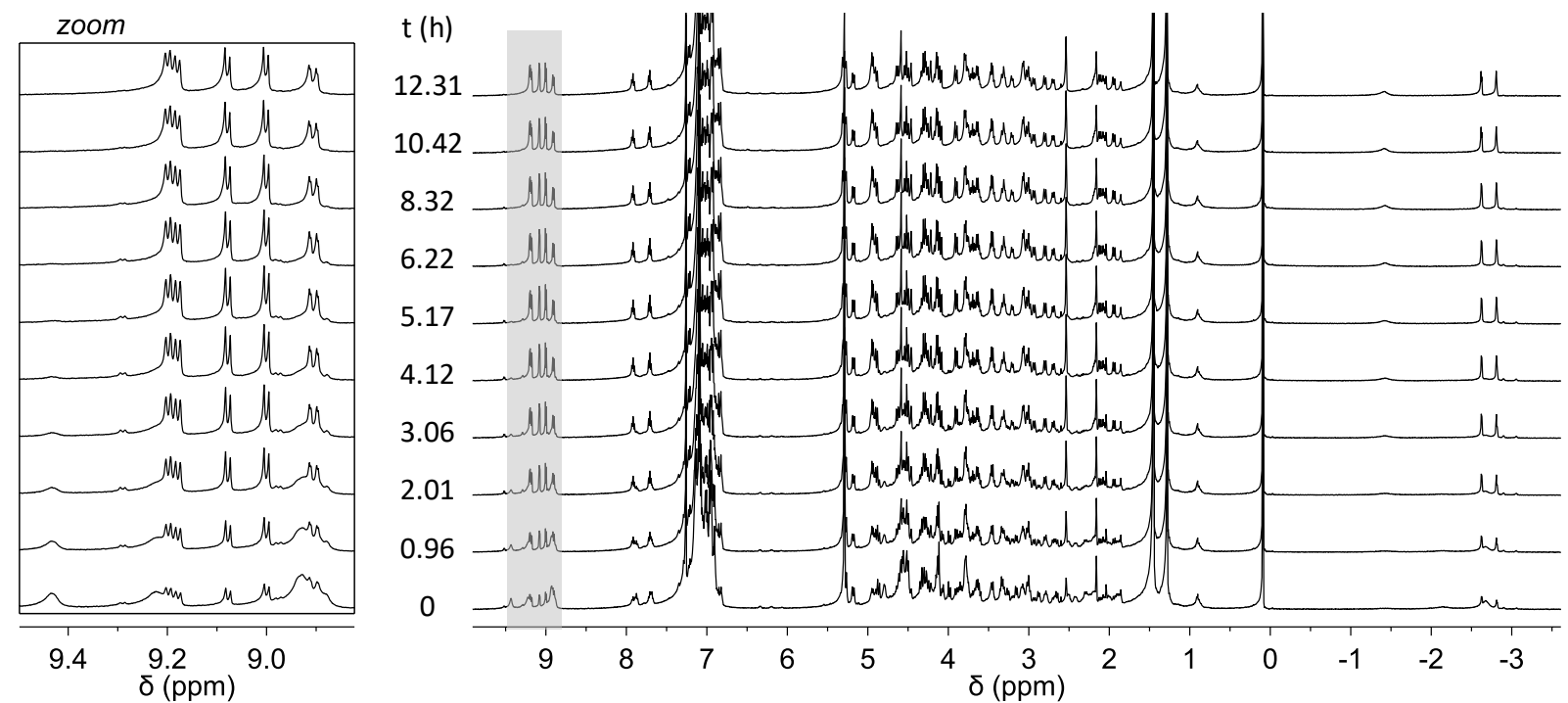

\subsubsection{Kinetic analysis of the conformational isomerization}

The isolated protons signals of ${ }^{\mathrm{R}}[\mathbf{2 6}] \mathbf{3}$ were broadened over the whole isomerization process at $330 \mathrm{~K}$. The corresponding integral measurements were thus used to approximate the isomerization barrier between ${ }^{\mathrm{R}}[\mathbf{2 6}] \mathbf{3}$ and ${ }^{\mathrm{R}}[\mathbf{2 6}] \mathbf{1}$. In this case, the equilibrated model fits better to the collected data. We thus considered the following equilibrium.

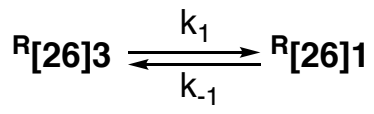

Rate $=d\left[{ }^{R}[26] 1\right] / d t=-d\left[{ }^{R}[26] 3\right] / d t=k_{1}\left[{ }^{R}[26] 3\right]-k_{-1}\left[{ }^{R}[26] 1\right]$

Isomerization in $\mathrm{CDCl}_{3}(330 \mathrm{~K})$

Analysis of the kinetic profiles with Dynafit software gave the kinetic constants $\mathrm{k}_{1}=0.34 \mathrm{~h}^{-1}$ and $\mathrm{k}_{-1}=0.03$ $\mathrm{h}^{-1}$ from ${ }^{1} \mathrm{H}$ NMR data at $330 \mathrm{~K}$ in $\mathrm{CDCl}_{3}$ (signal at 9.43 ppm for ${ }^{\mathrm{R}}[26] 3$ and signal at 9.08 ppm for ${ }^{\mathrm{R}}[26] 1$, see Part 4.4.1).

Eyring's equation was used to calculate free energy of activation $\left(\Delta G^{\ddagger}\right)$ of the isomerization process:

$k=\frac{k_{B} T}{h} e^{\left(\frac{-\Delta G^{\ddagger}}{R T}\right)}$

$\mathrm{k}_{B}$ is Boltzmann's constant $\left(\mathrm{k}_{B}=1.3806 \times 10^{-23} \mathrm{~J} \cdot \mathrm{K}^{-1}\right)$

$h$ is Planck's constant $\left(h=6.626 \times 10^{-34} \mathrm{~J} . \mathrm{s}\right)$

$R$ is the gaz constant $\left(R=8.314 \mathrm{~J} \cdot \mathrm{K}^{-1} \cdot \mathrm{mol}^{-1}\right)$

$\Delta \mathrm{G}^{\ddagger}{ }_{1}=25.46 \mathrm{kcal} . \mathrm{mol}^{-1}$ and $\Delta \mathrm{G}^{\ddagger}{ }_{-1}=27.03 \mathrm{kcal} \cdot \mathrm{mol}^{-1}$

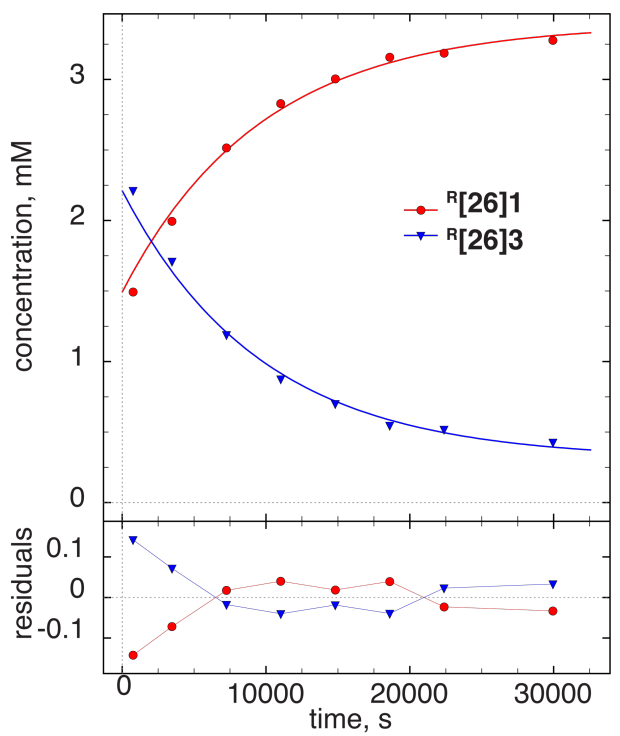




\section{$5 \quad$ NMR Spectra}

5.1 Cyclodextrin-bis acetal 8

5.1.1 ${ }^{1} \mathrm{HNMR}\left(\mathrm{CDCl}_{3}, 298 \mathrm{~K}, 500 \mathrm{MHz}\right)$
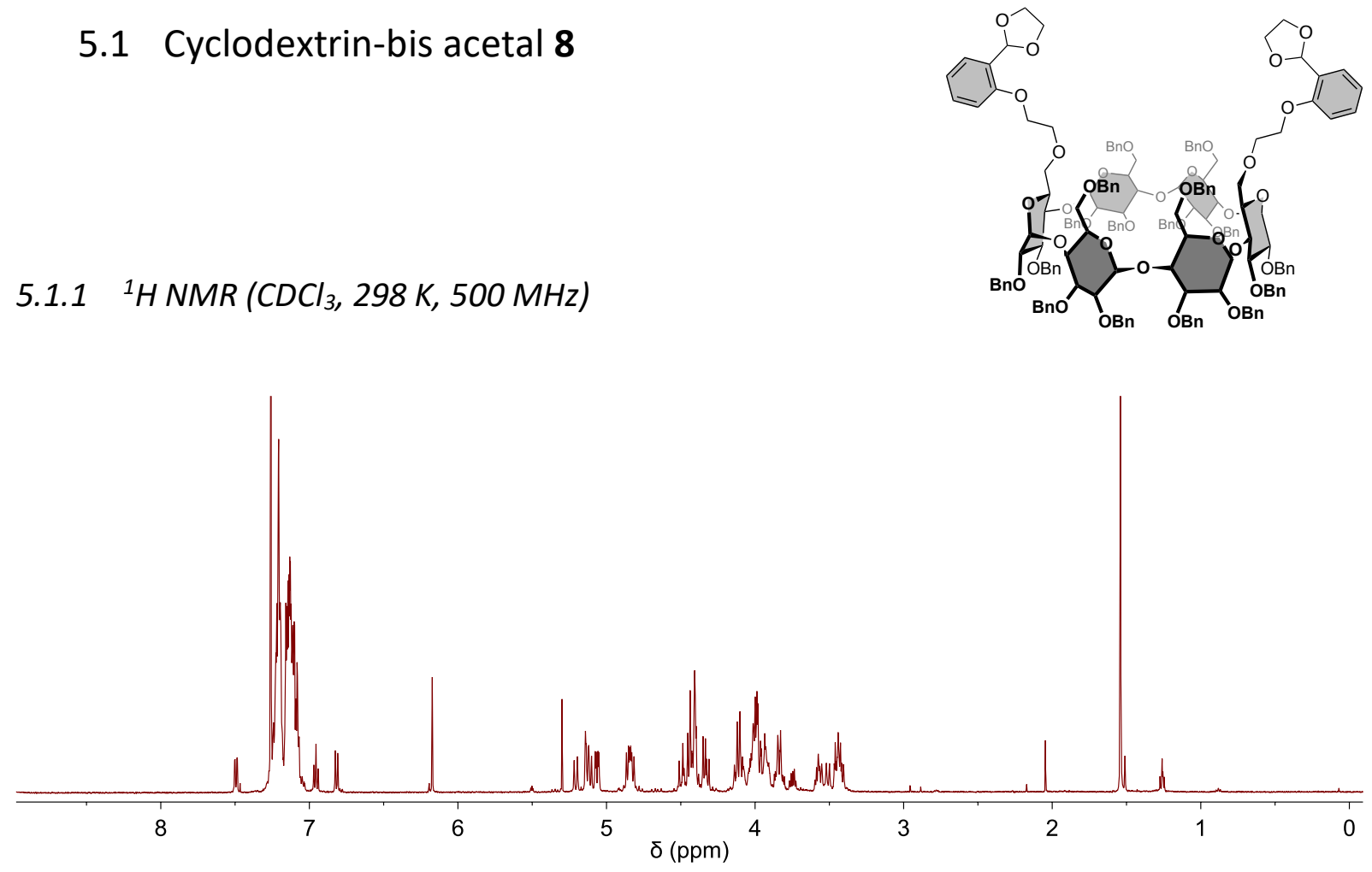
5.1.2 2D COSY-DQF $\left(\mathrm{CDCl}_{3}, 298 \mathrm{~K}, 500 \mathrm{MHz}\right)$
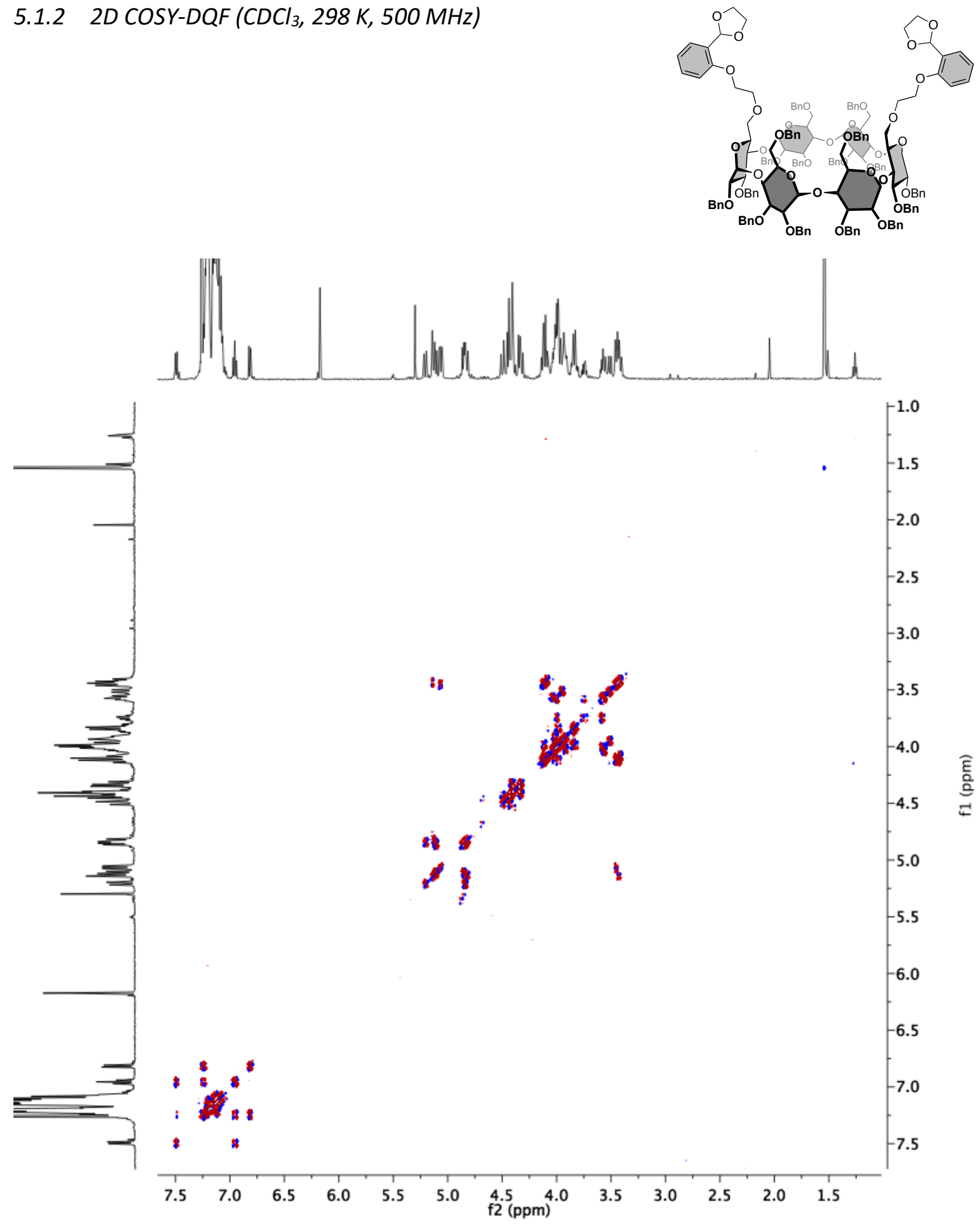
5.1.3 2D TOCSY $\left(\mathrm{CDCl}_{3}, 298 \mathrm{~K}, 500 \mathrm{MHz}, \tau \mathrm{m}=240 \mathrm{~ms}\right)$
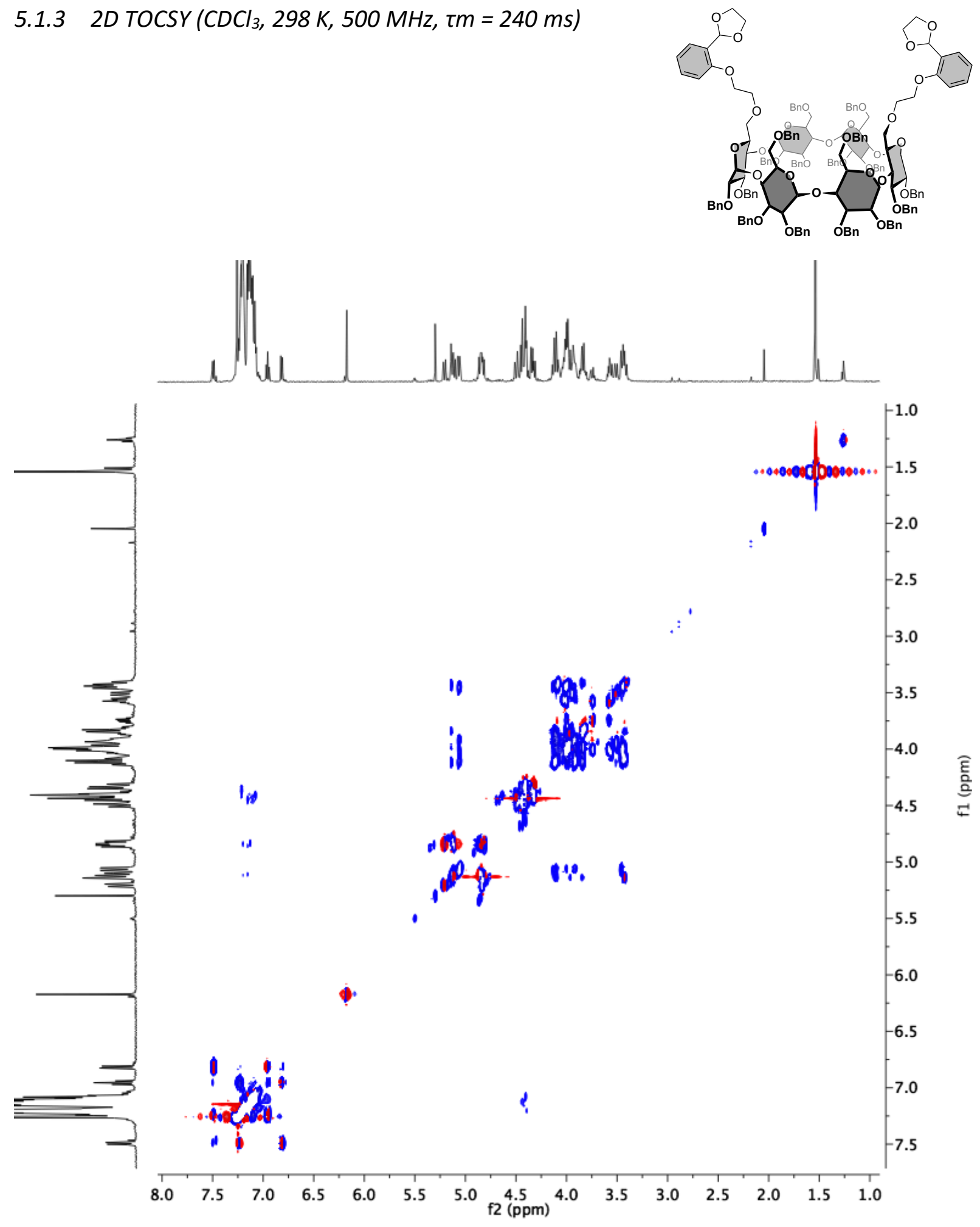
5.1.4 2D HSQC-edited $\left(\mathrm{CDCl}_{3}, 298 \mathrm{~K}, 500 \mathrm{MHz}\right)$
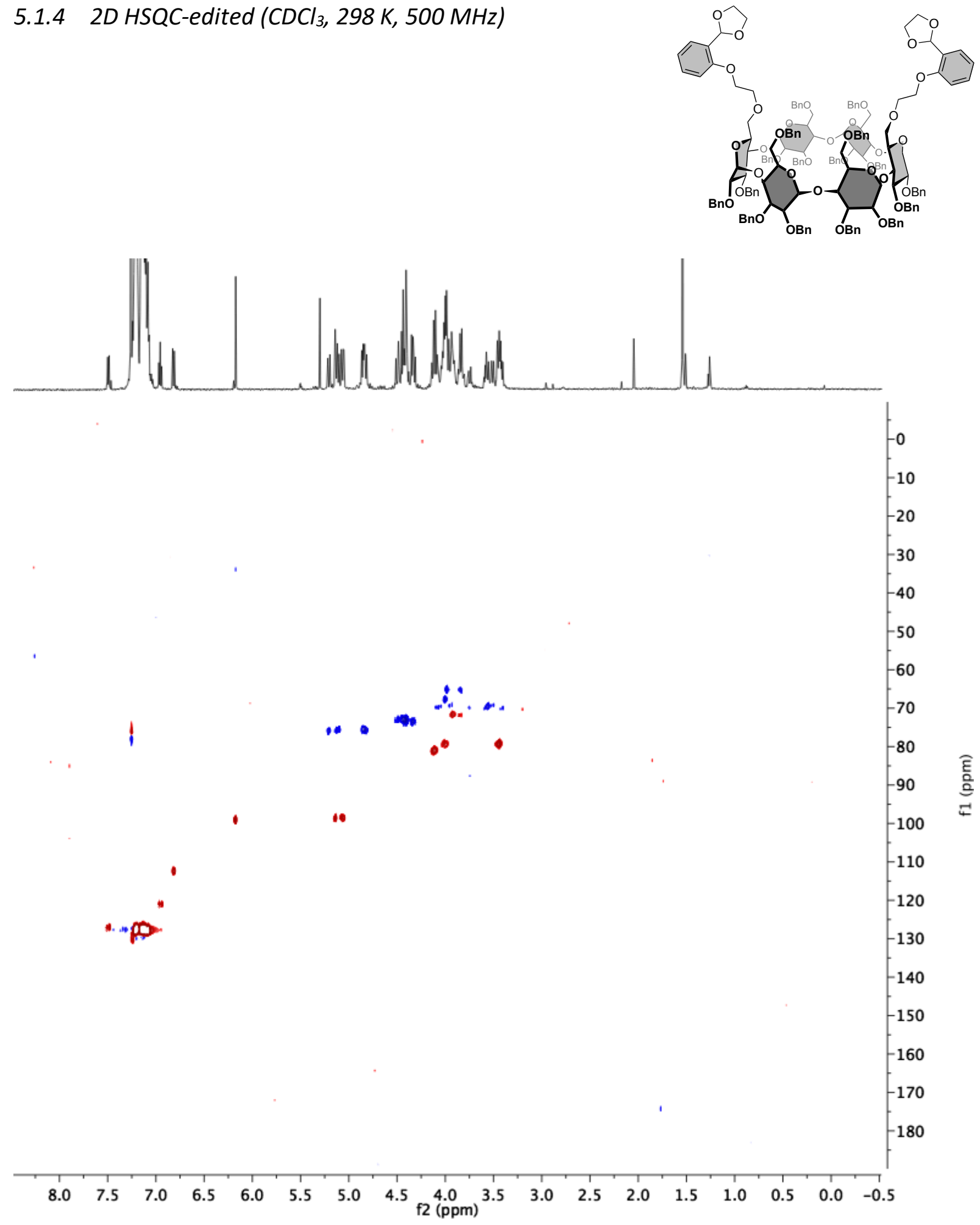
5.1.5 2D NOESY $\left(\mathrm{CDCl}_{3}, 298 \mathrm{~K}, 500 \mathrm{MHz}, \mathrm{\tau m}=800 \mathrm{~ms}\right)$
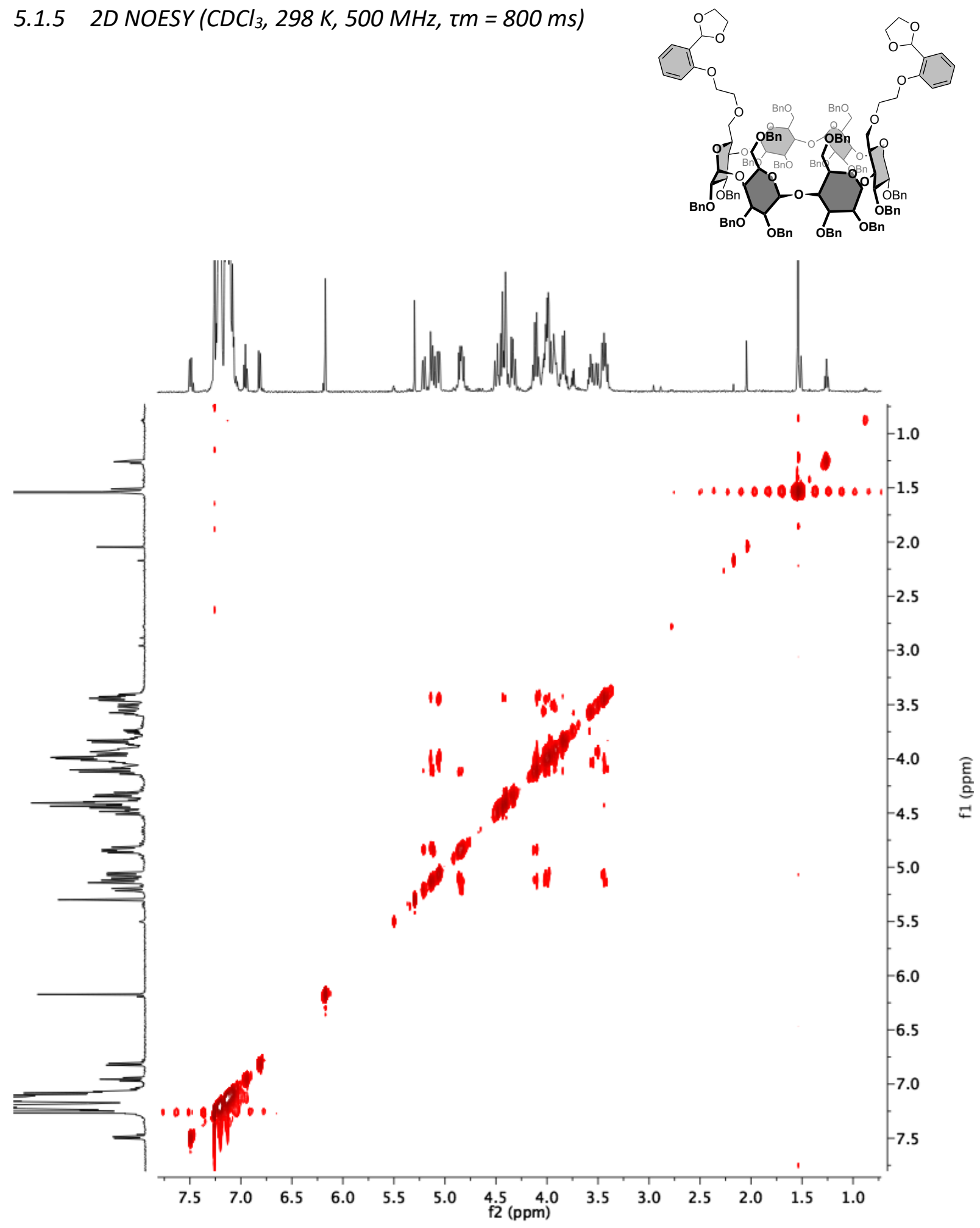
5.2 Cyclodextrin-bis aldehyde $\mathbf{5}$

5.2.1 ${ }^{1} \mathrm{H} \mathrm{NMR}\left(\mathrm{CDCl}_{3}, 298 \mathrm{~K}, 500 \mathrm{MHz}\right)$
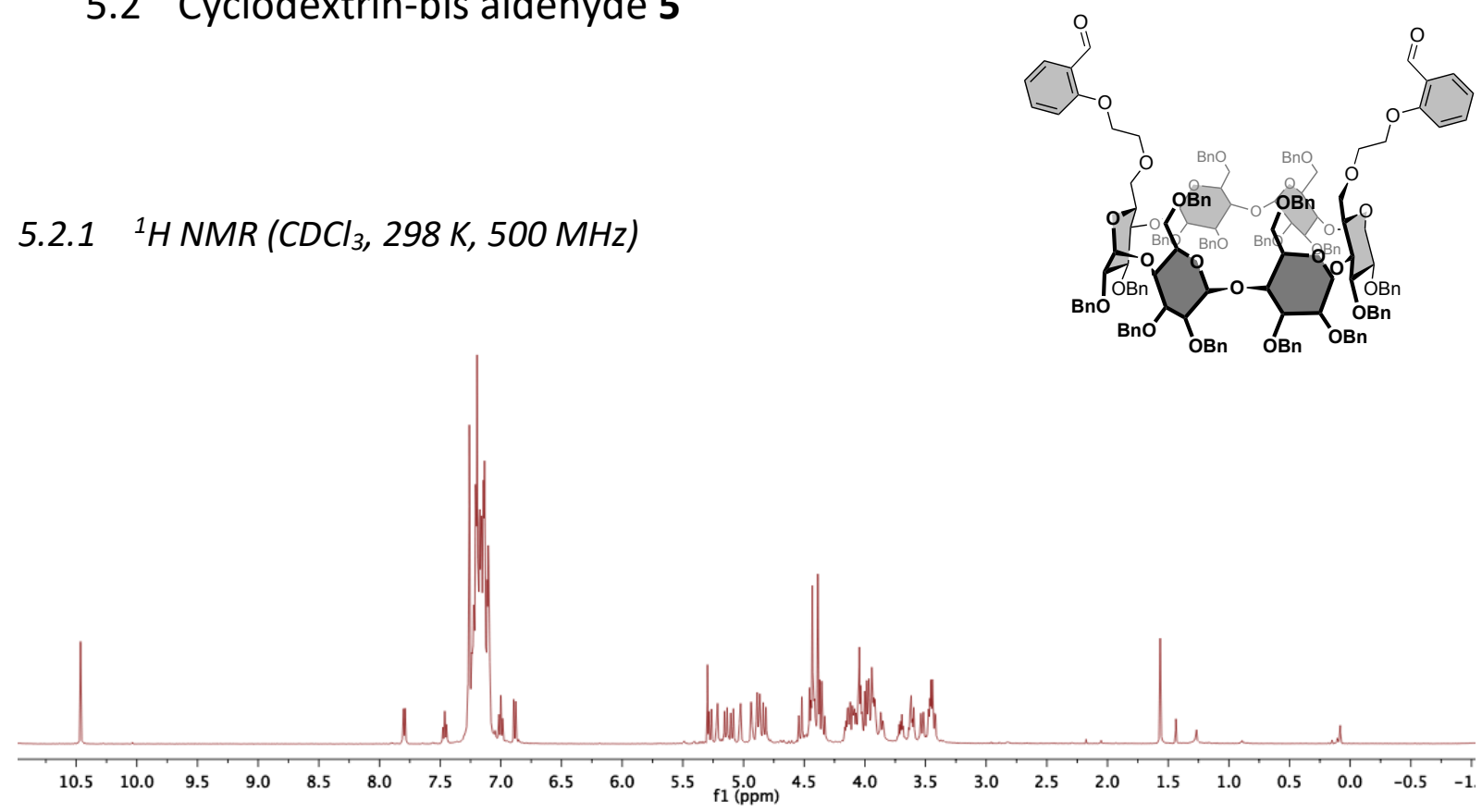

5.2.2 ${ }^{13} \mathrm{CNMR}\left(\mathrm{CDCl}_{3}, 298 \mathrm{~K}, 125 \mathrm{MHz}\right)$

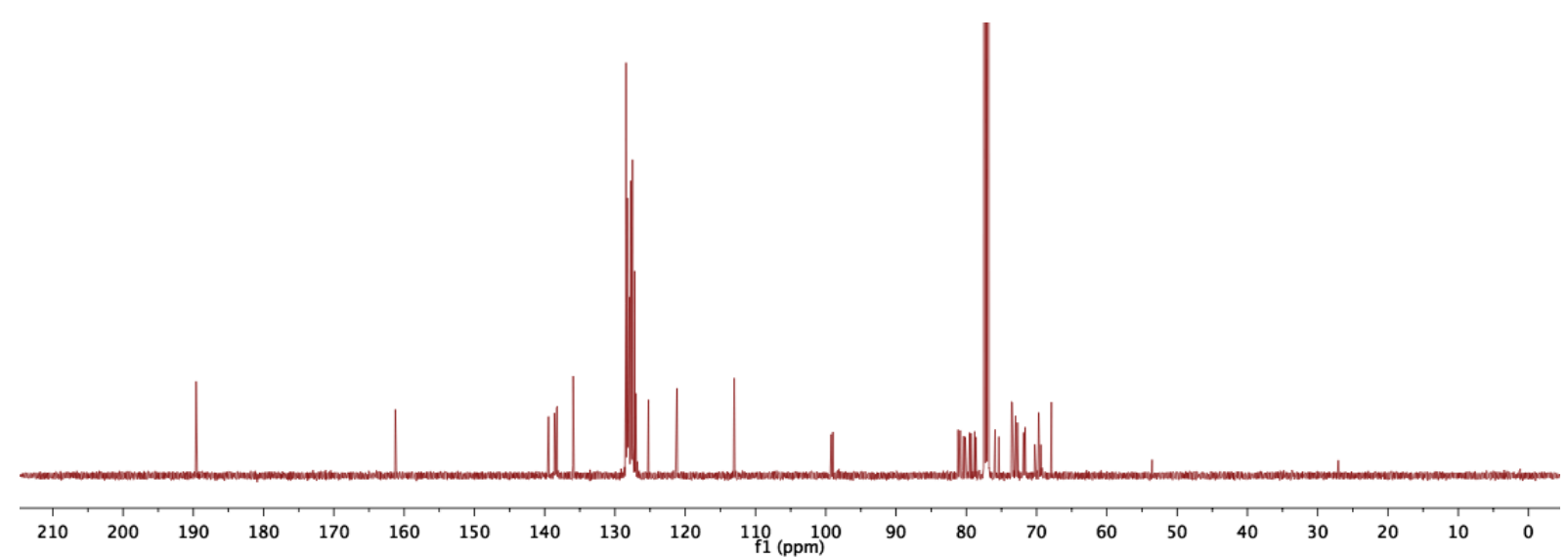


5.2.3 $2 D \operatorname{cosY}\left(C D C l_{3}, 298 \mathrm{~K}, 500 \mathrm{MHz}\right)$
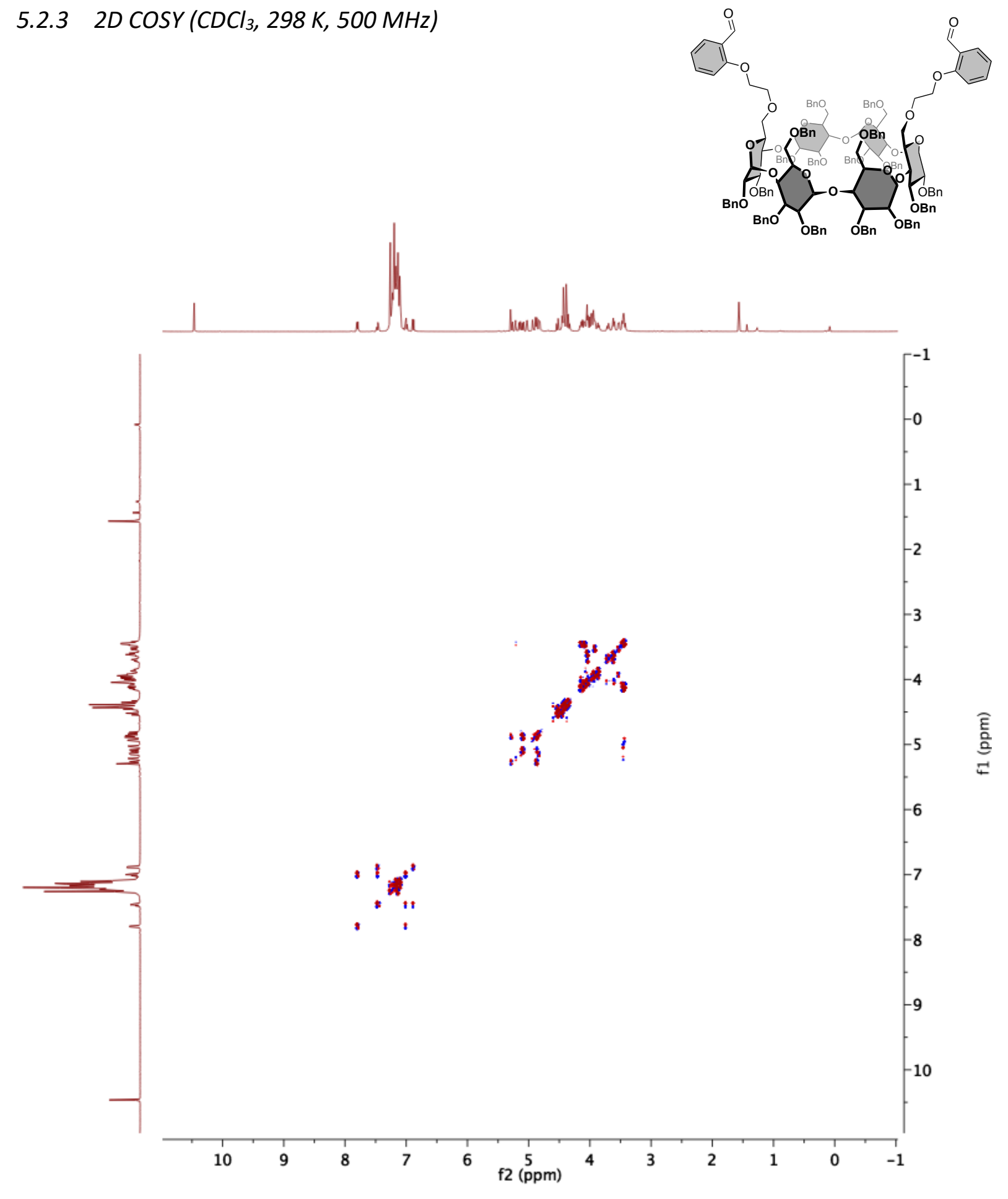
5.2.4 2D TOCSY $\left(\mathrm{CDCl}_{3}, 298 \mathrm{~K}, 500 \mathrm{MHz}, \tau m=240 \mathrm{~ms}\right)$
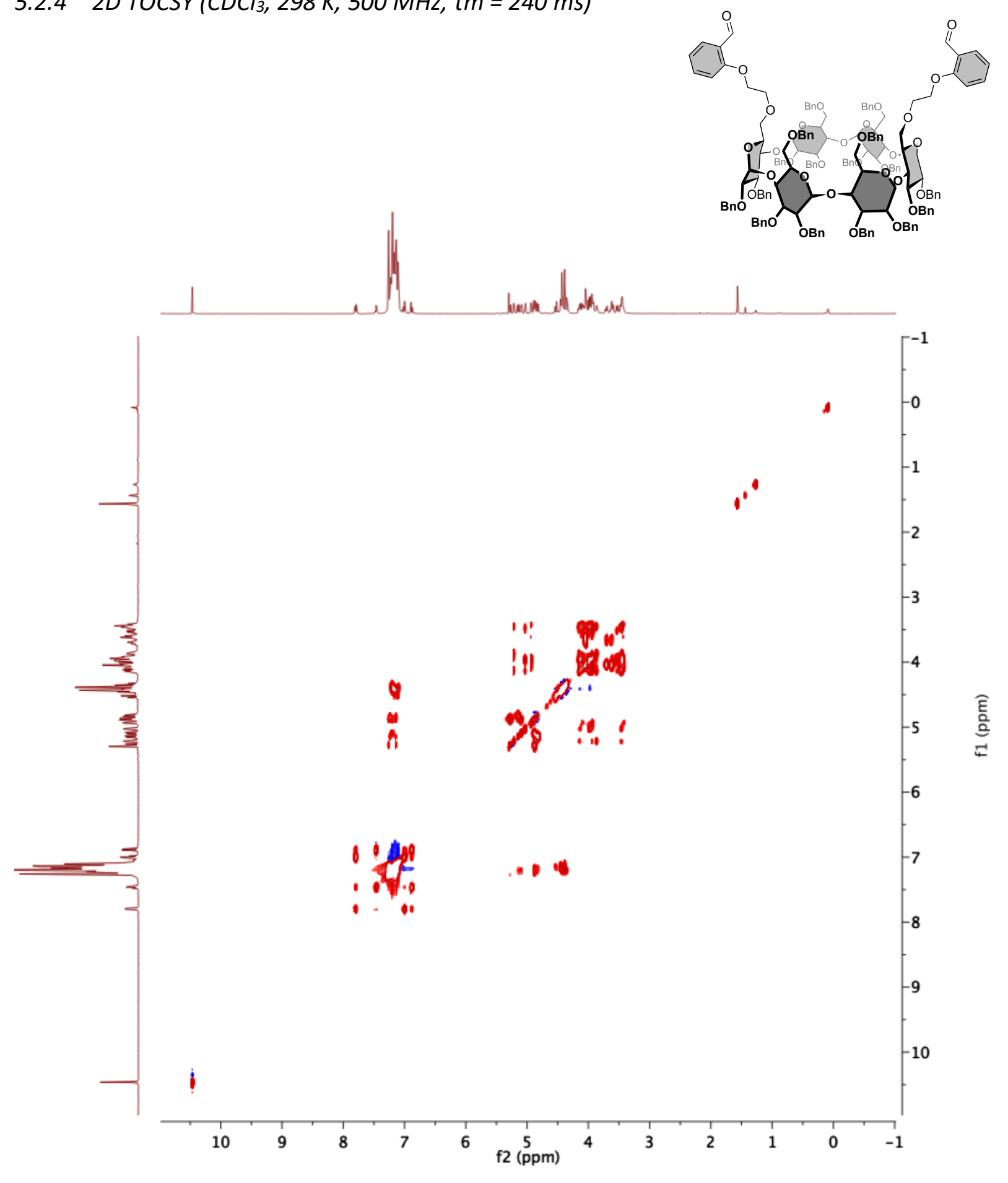
5.2.5 2D HSQC-edited ( $\left.\mathrm{CDCl}_{3}, 298 \mathrm{~K}, 500 \mathrm{MHz}\right)$
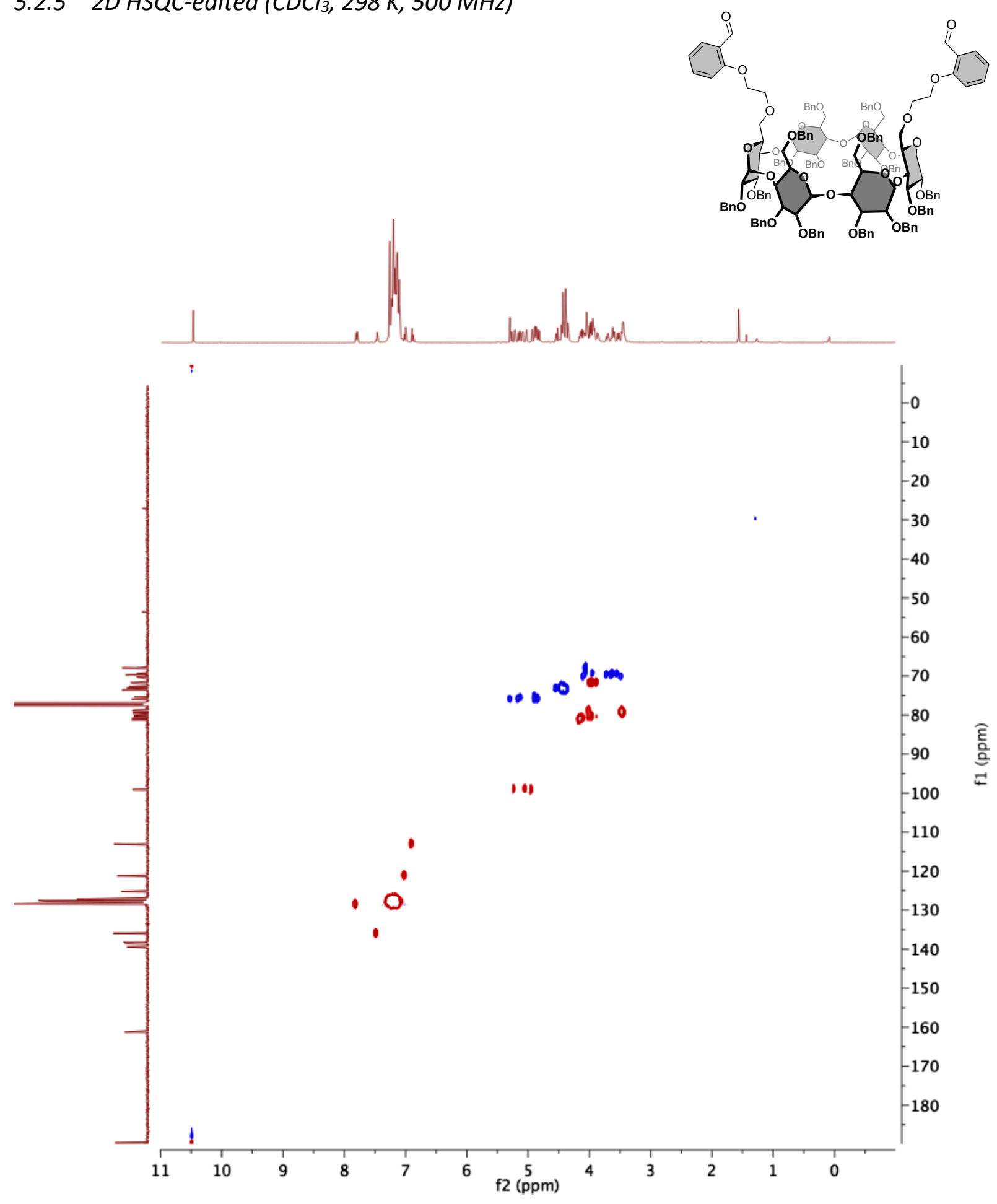
5.2.6 2D NOESY $\left(\mathrm{CDCl}_{3}, 298 \mathrm{~K}, 500 \mathrm{MHz}, \mathrm{\tau m}=800 \mathrm{~ms}\right)$
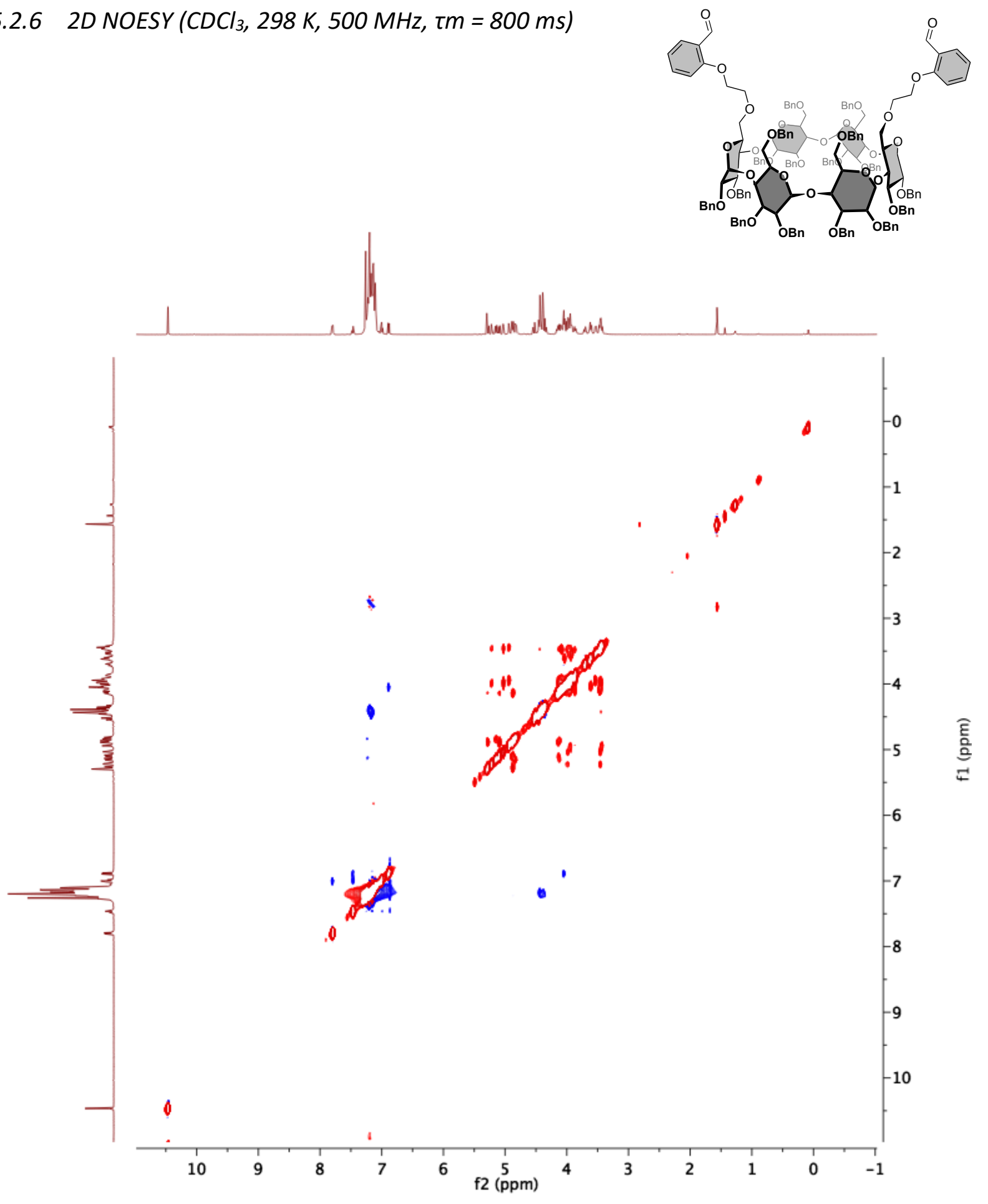
5.2.7 2D ROESY $\left(\mathrm{CDCl}_{3}, 298 \mathrm{~K}, 500 \mathrm{MHz}, \tau \mathrm{m}=500 \mathrm{~ms}\right)$
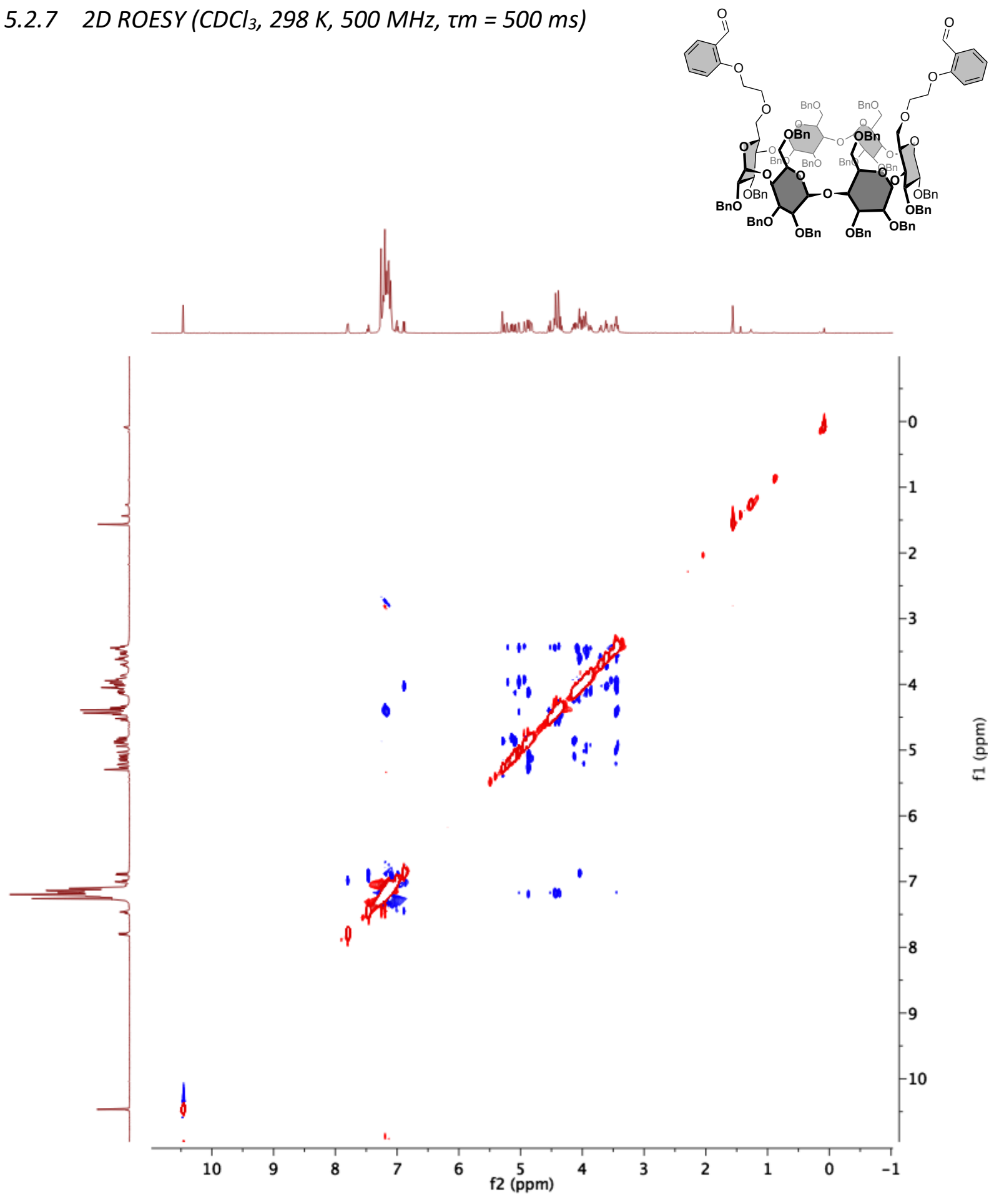


\section{$5.3{ }^{\mathrm{R}}[26] 1$}

5.3.1 ${ }^{1} \mathrm{HNMR}\left(\mathrm{CDCl}_{3}, 298 \mathrm{~K}, 500 \mathrm{MHz}\right)$

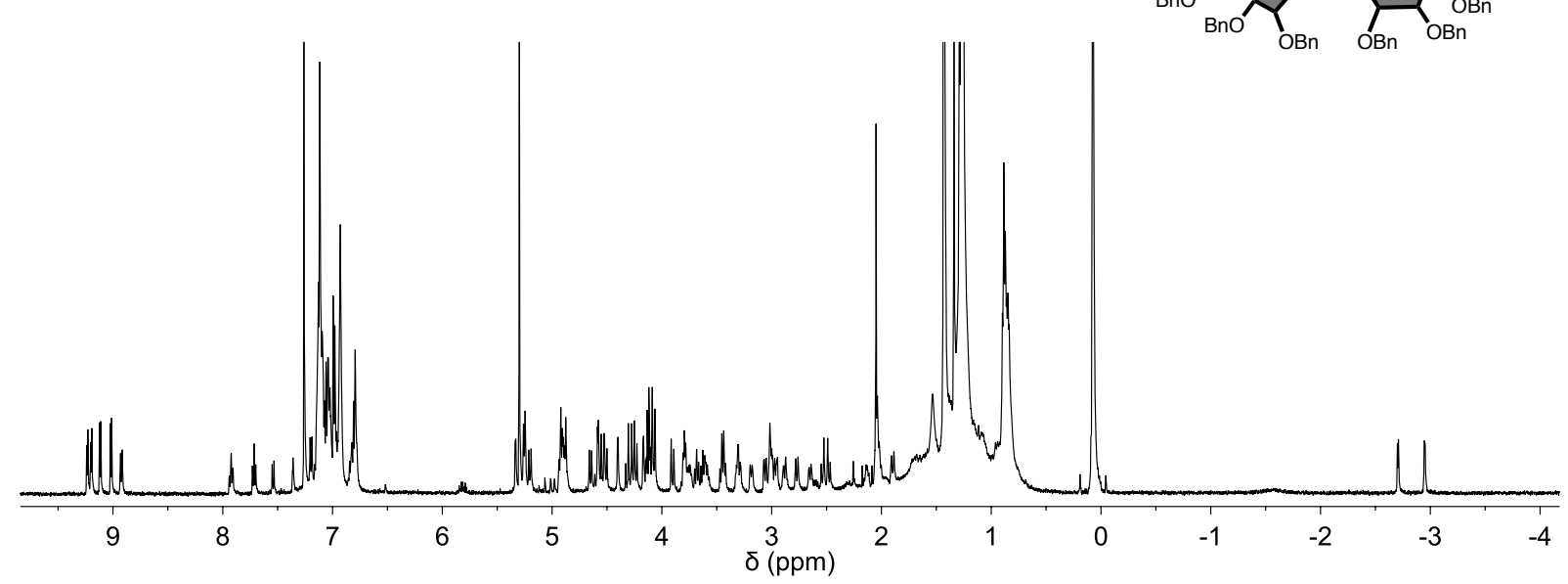

5.3.2 ${ }^{19} \mathrm{FNMR}\left(\mathrm{CDCl}_{3}, 298 \mathrm{~K}, 376 \mathrm{MHz}\right)$

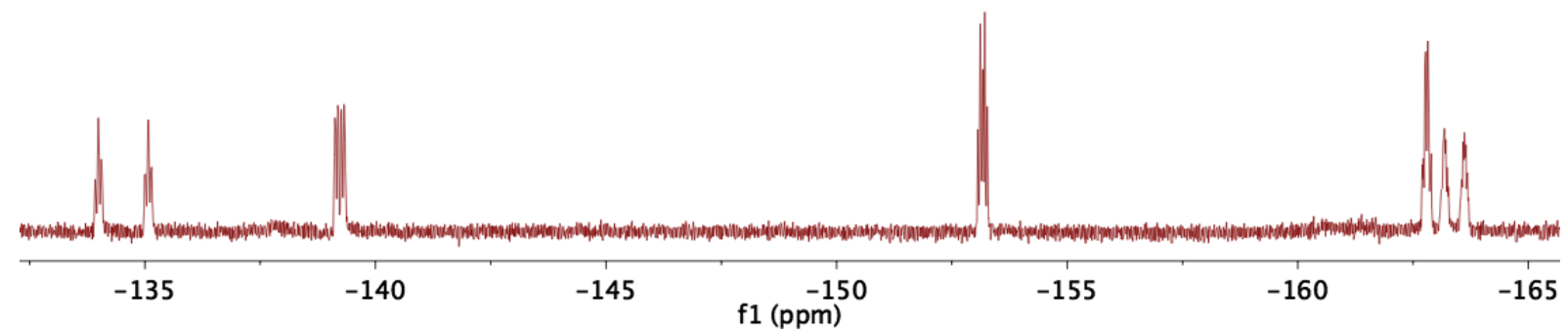


5.3.3 $2 D \operatorname{COSY}^{19} \mathrm{~F}-{ }^{19} \mathrm{~F}\left(\mathrm{CDCl}_{3}, 298 \mathrm{~K}, 376 \mathrm{MHz}\right)$
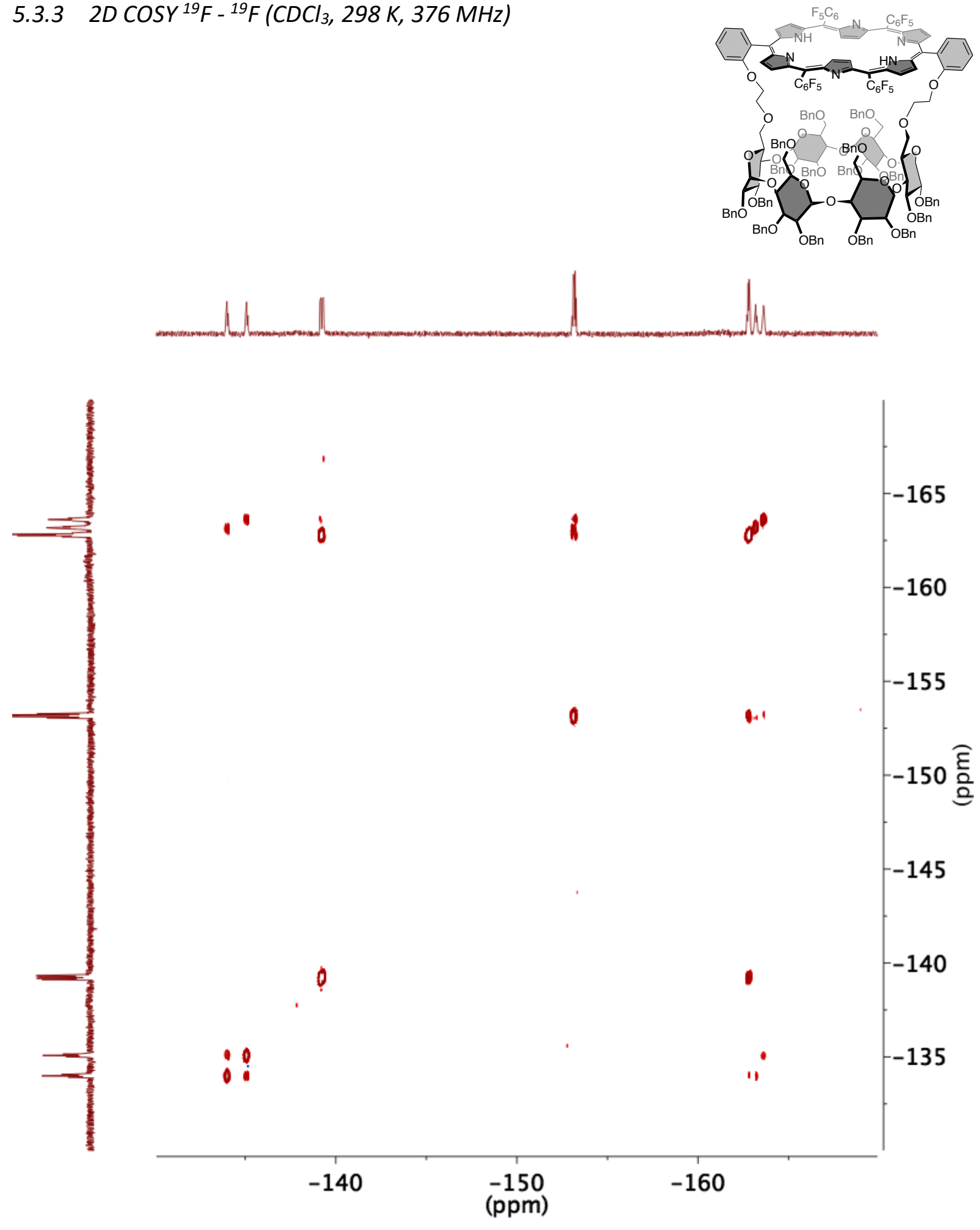
5.3.4 2D COSY-DQF $\left(\mathrm{CDCl}_{3}, 298 \mathrm{~K}, 500 \mathrm{MHz}\right)$
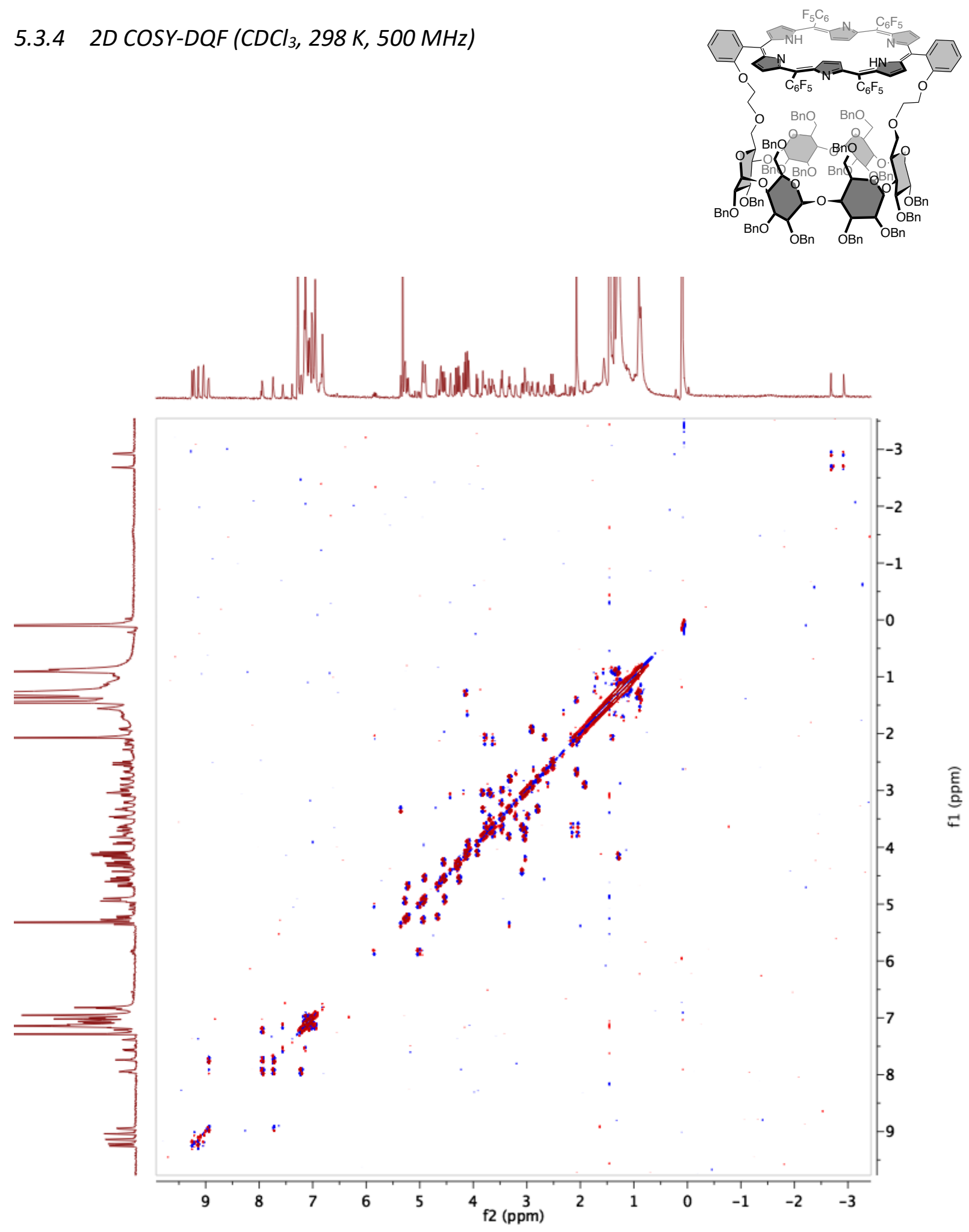
5.3.5 2D TOCSY $\left(\mathrm{CDCl}_{3}, 298 \mathrm{~K}, 500 \mathrm{MHz}, \tau m=240 \mathrm{~ms}\right)$
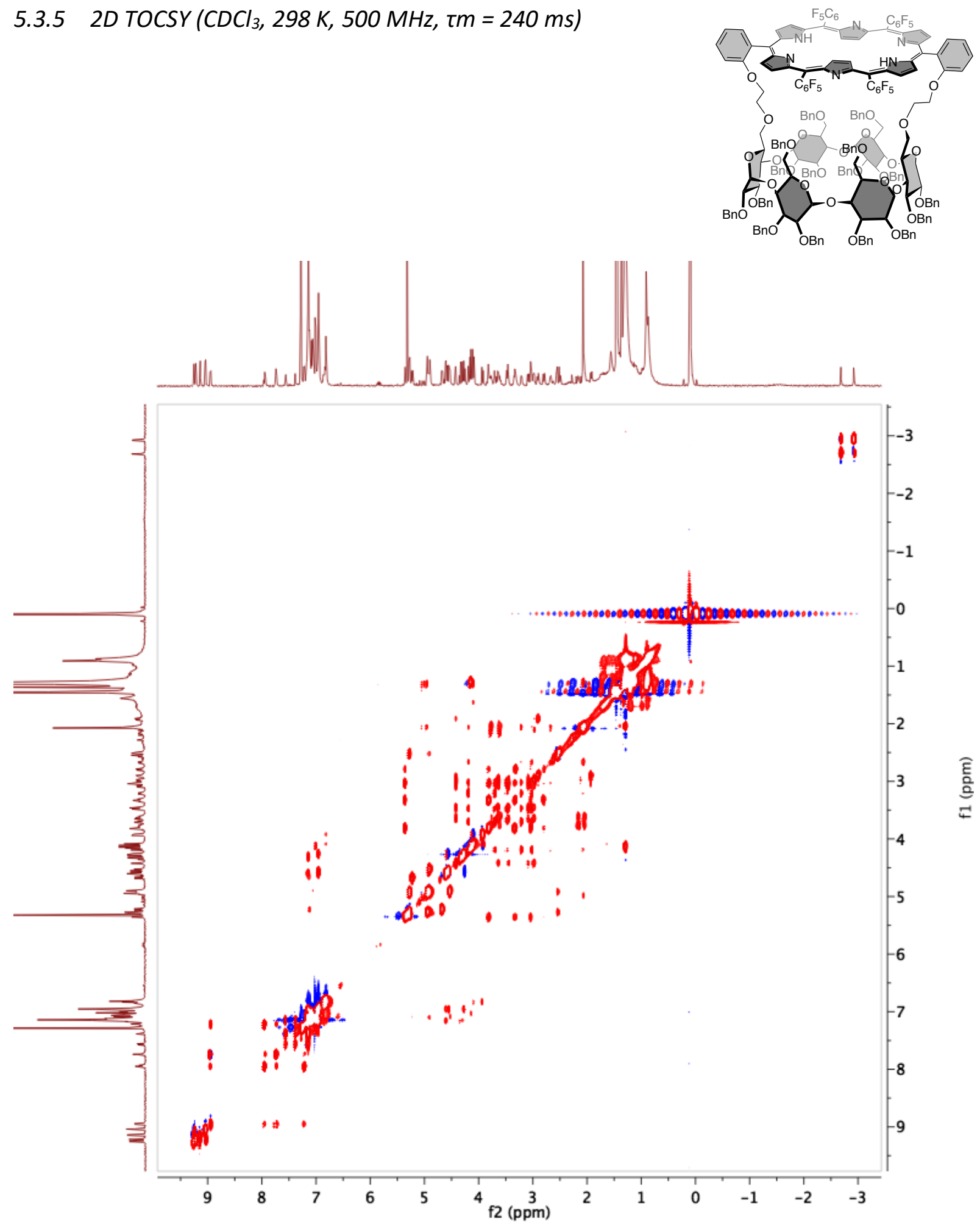
5.3.6 2D HSQC-edited (CDCl $, 298 \mathrm{~K}, 500 \mathrm{MHz})$
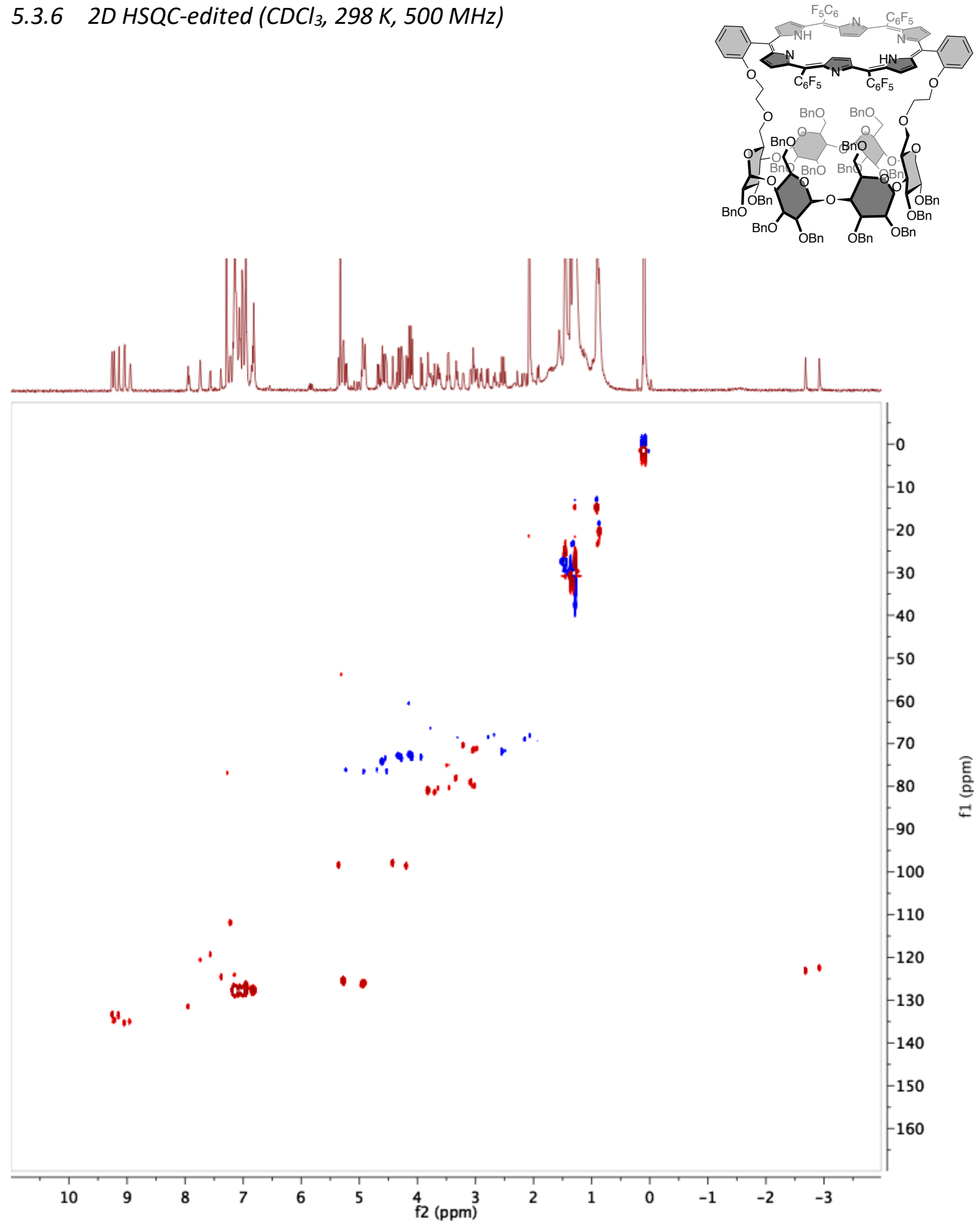
5.3.7 2D NOESY $\left(\mathrm{CDCl}_{3}, 298 \mathrm{~K}, 500 \mathrm{MHz}, \tau \mathrm{m}=800 \mathrm{~ms}\right)$
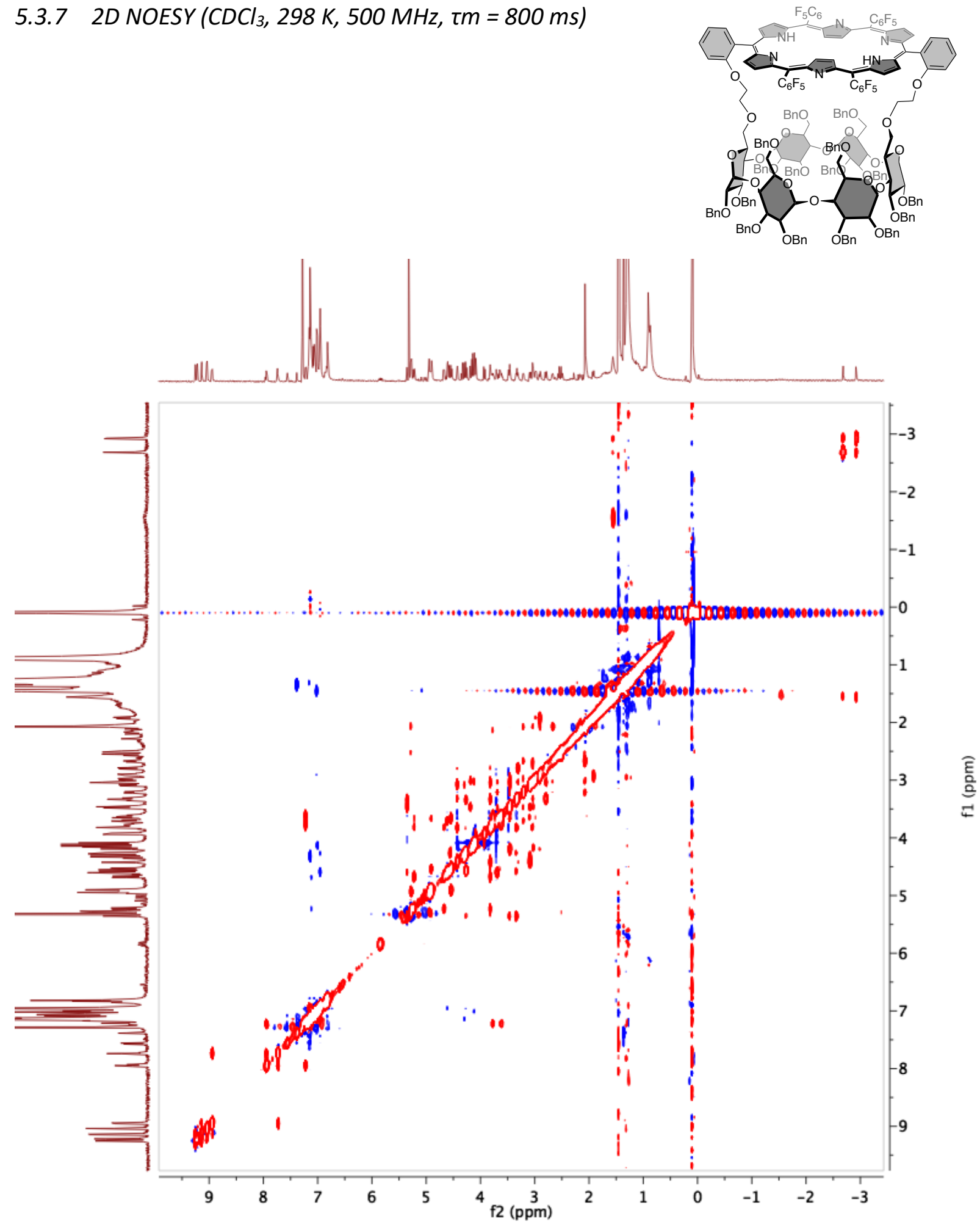


\section{$5.4{ }^{\mathrm{R}}[26] 2$}

\subsection{1 ${ }^{1} \mathrm{H} \mathrm{NMR}\left(\mathrm{CDCl}_{3}, 298 \mathrm{~K}, 500 \mathrm{MHz}\right)$}
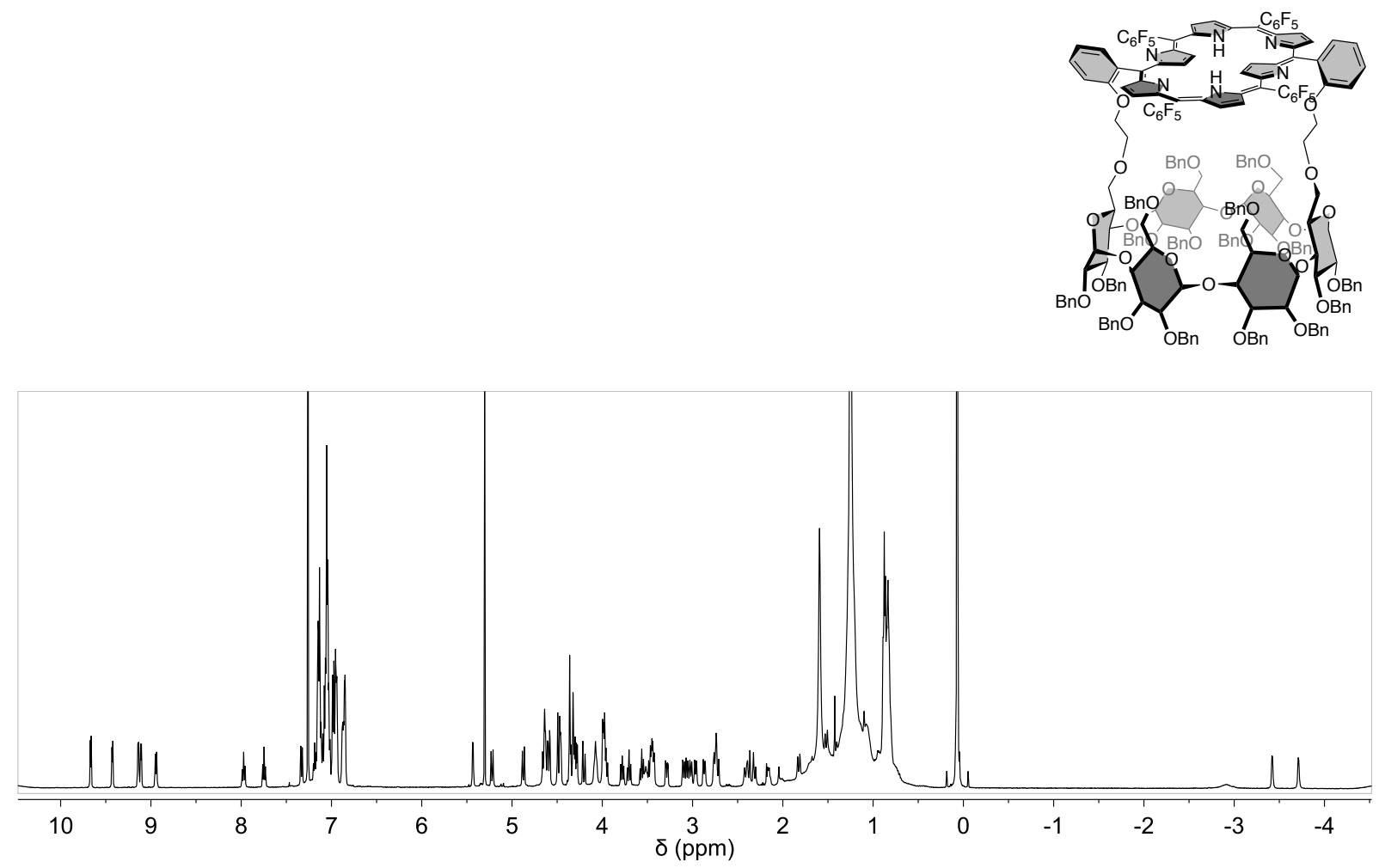

5.4.2 ${ }^{19} \mathrm{FNMR}\left(\mathrm{CDCl}_{3}, 298 \mathrm{~K}, 376 \mathrm{MHz}\right)$

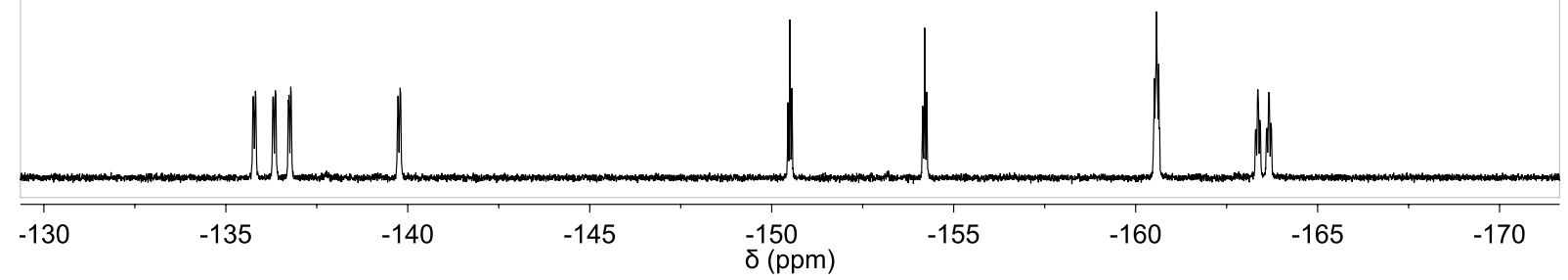


5.4.3 $2 D \operatorname{COSY}\left(\mathrm{CDCl}_{3}, 283 \mathrm{~K}, 500 \mathrm{MHz}\right)$

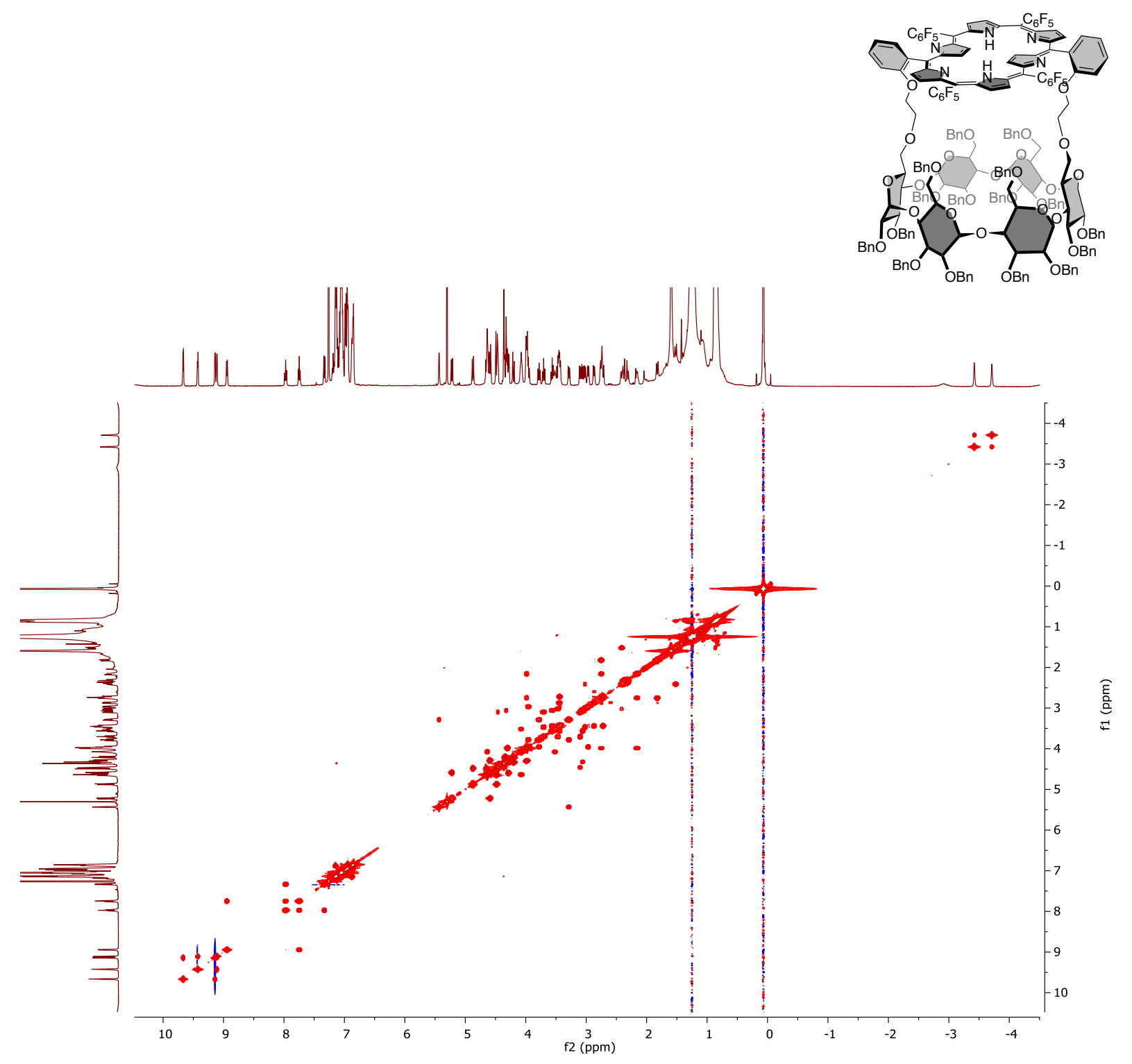


5.4.4 2D TOCSY $\left(\mathrm{CDCl}_{3}, 283 \mathrm{~K}, 500 \mathrm{MHz}, \tau m=240 \mathrm{~ms}\right)$

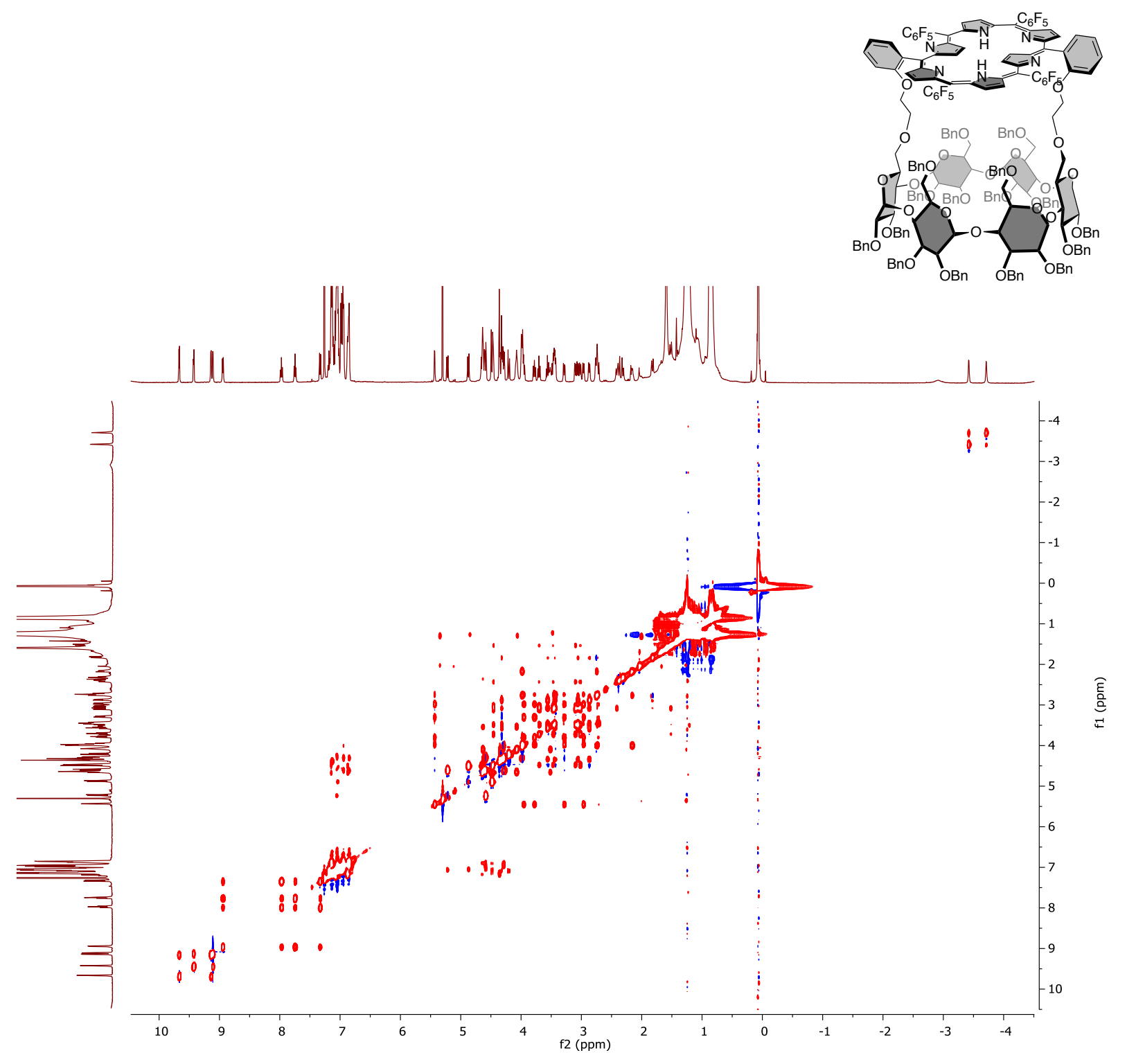


5.4.5 2D HSQC-edited ( $\left.\mathrm{CDCl}_{3}, 283 \mathrm{~K}, 500 \mathrm{MHz}\right)$
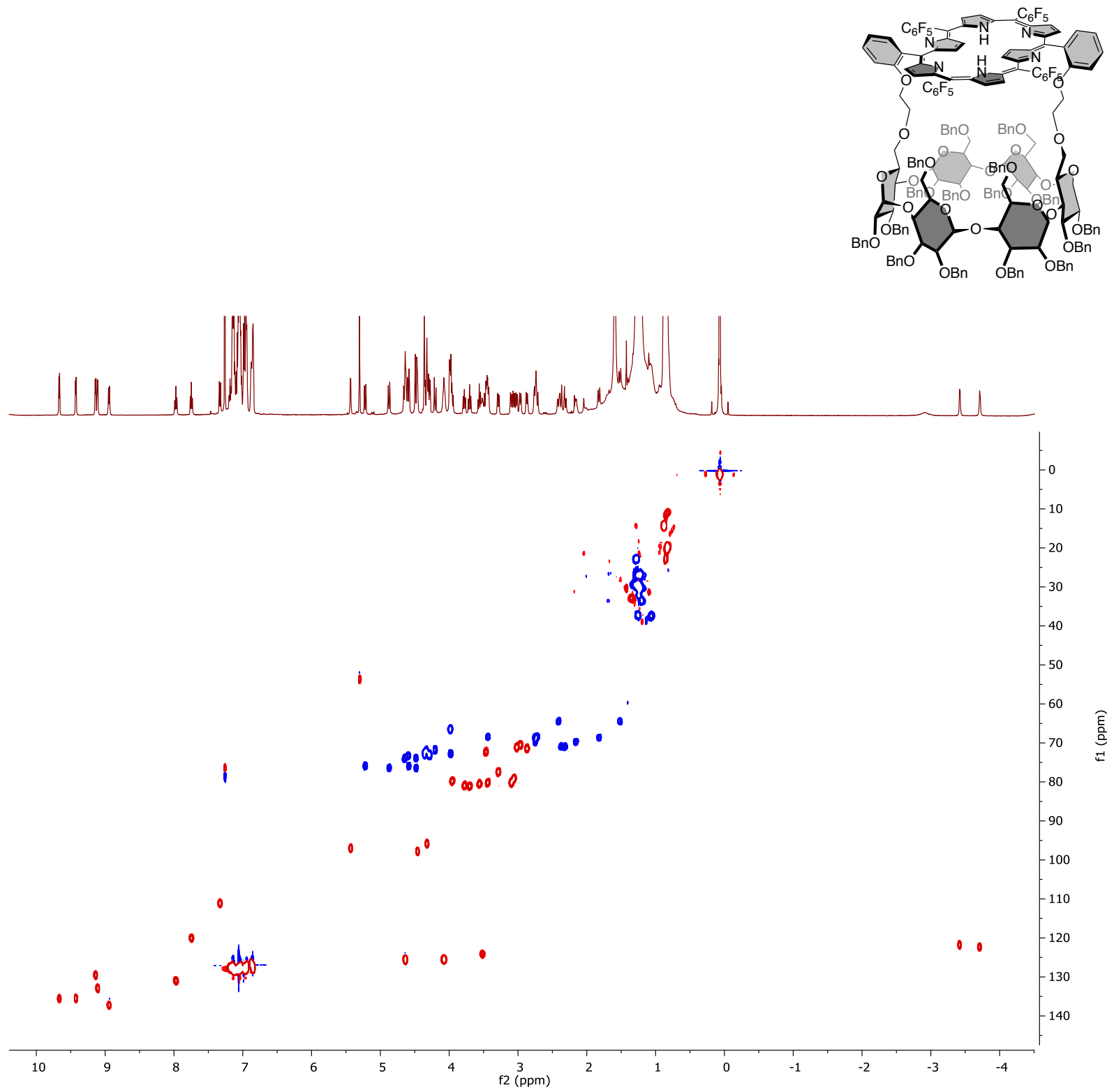

S38 
5.4.6 2D NOESY $\left(\mathrm{CDCl}_{3}, 283 \mathrm{~K}, 500 \mathrm{MHz}, \mathrm{\tau m}=800 \mathrm{~ms}\right)$
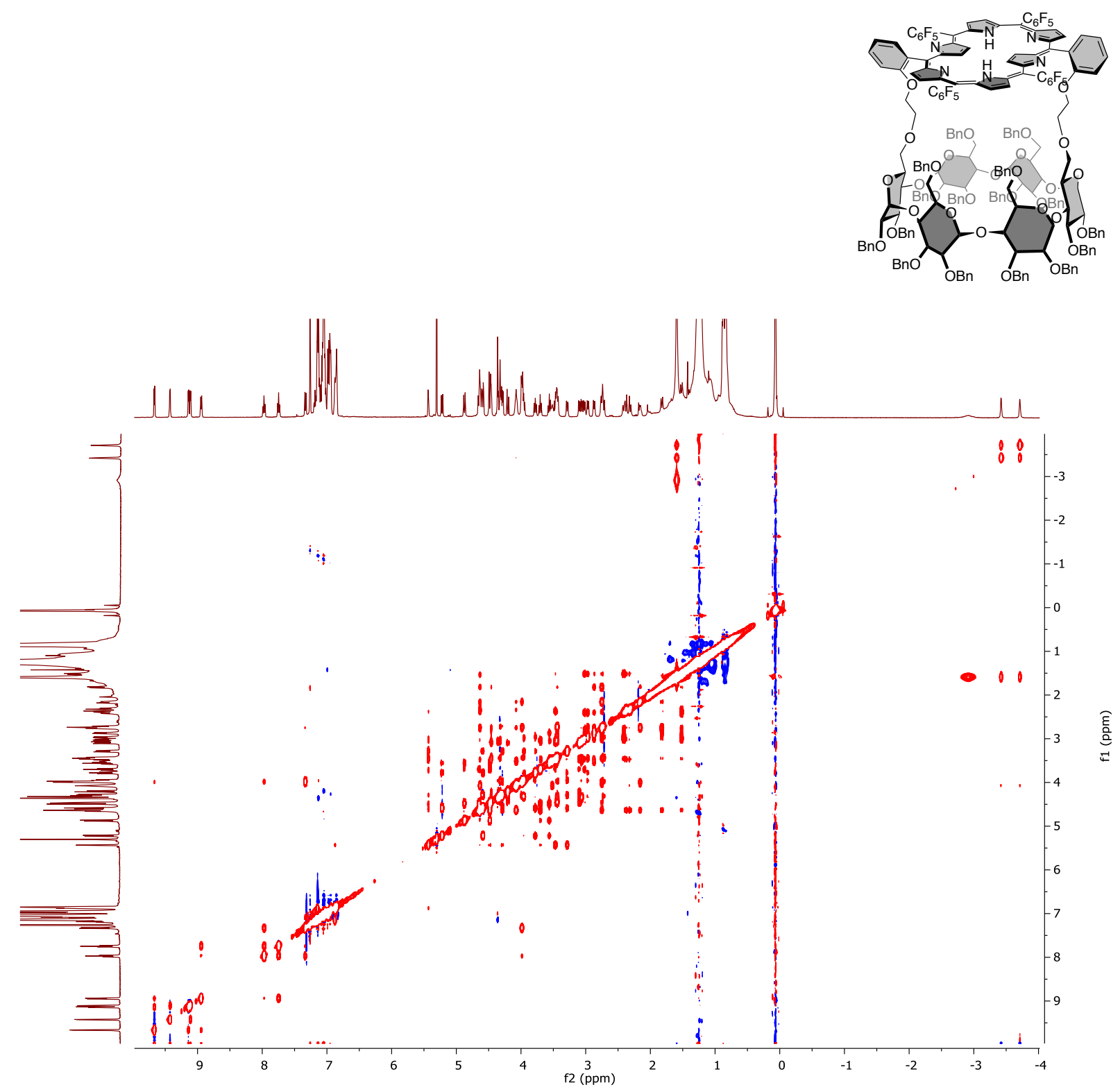
5.4.7 ${ }^{19} \mathrm{~F} 2 \mathrm{D} \operatorname{COSY}\left(\mathrm{CDCl}_{3}, 298 \mathrm{~K}, 376 \mathrm{MHz}\right)$

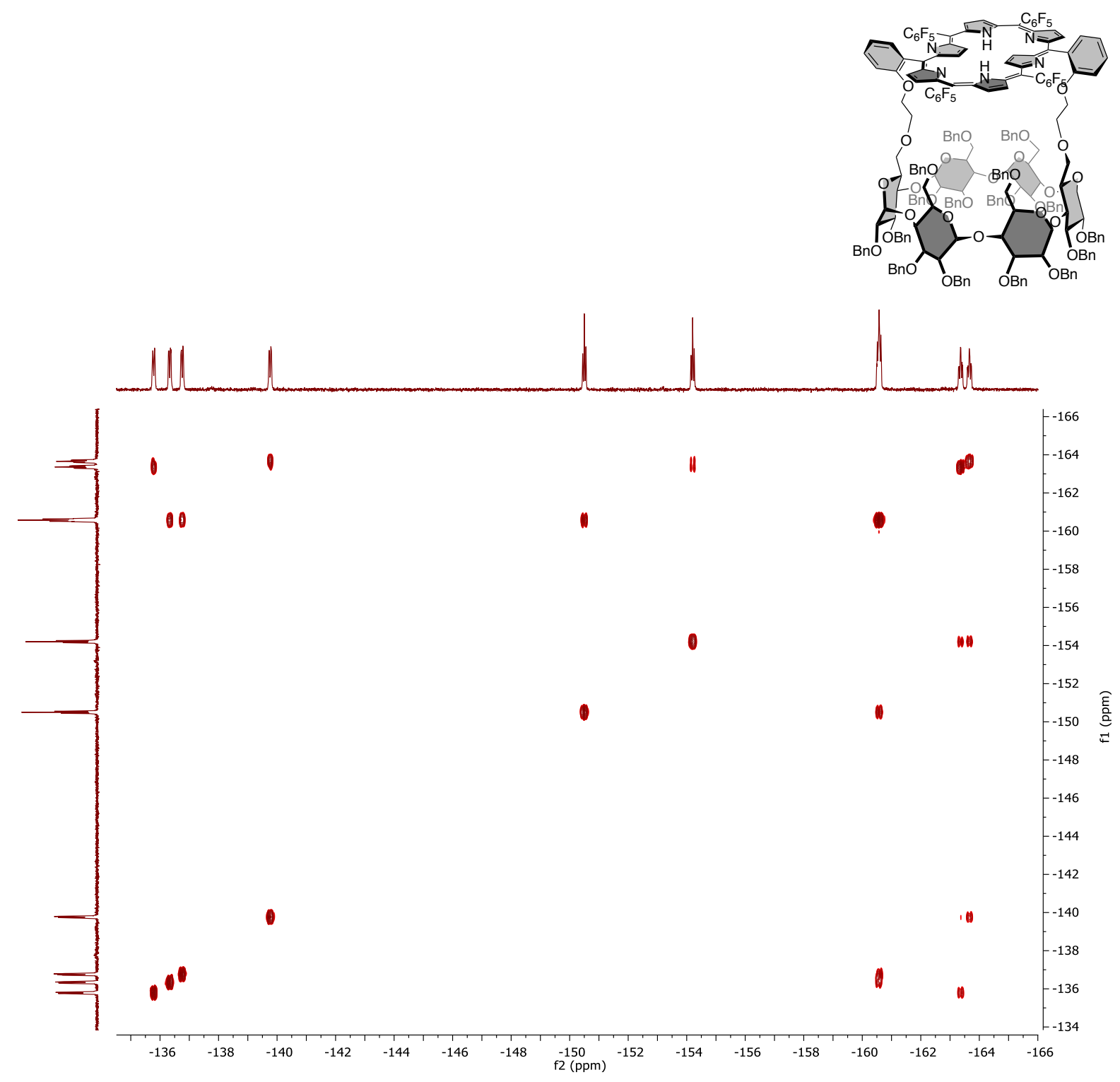




\section{$5.5{ }^{\mathrm{R}}[26] 3$}

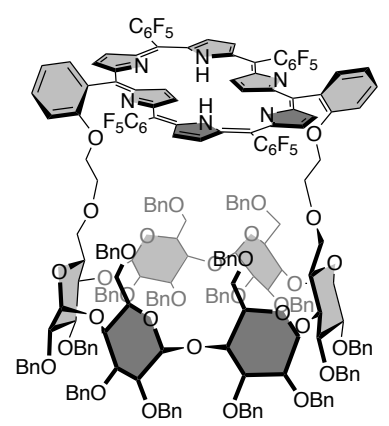

5.5.1 ${ }^{1} \mathrm{HNMR}\left(\mathrm{CDCl}_{3}, 283 \mathrm{~K}, 500 \mathrm{MHz}\right)$

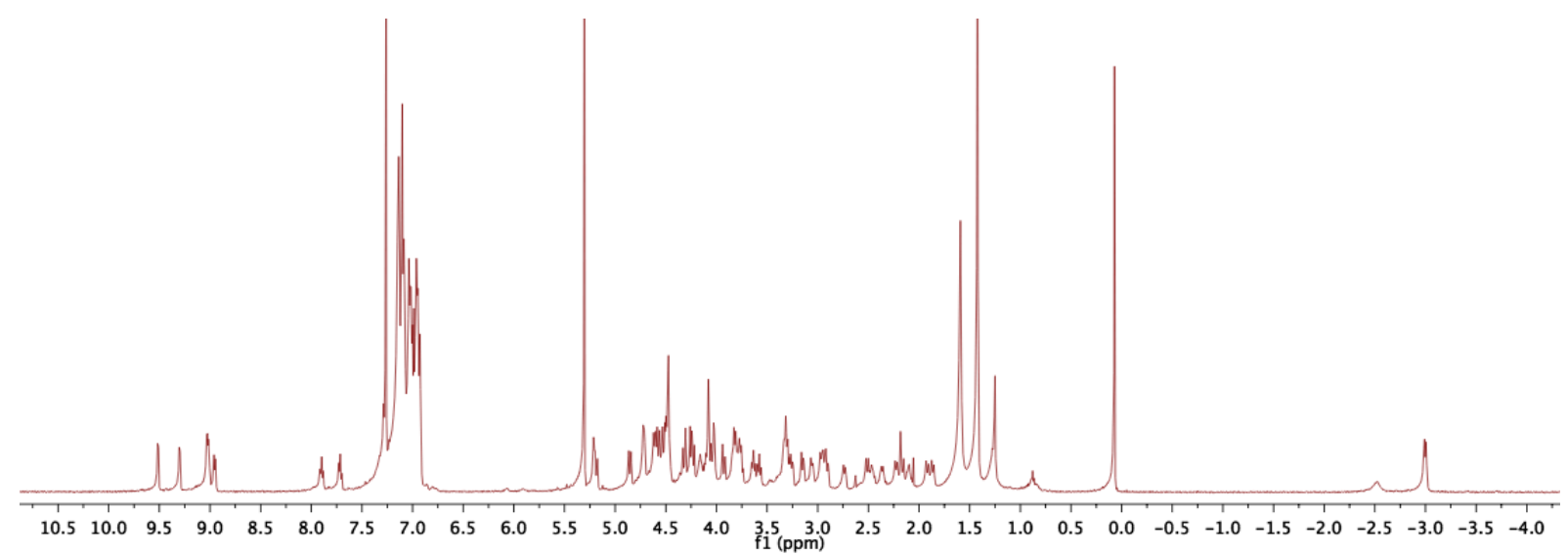

5.5.2 ${ }^{19} \mathrm{FNMR}\left(\mathrm{CDCl}_{3}, 298 \mathrm{~K}, 376 \mathrm{MHz}\right)$

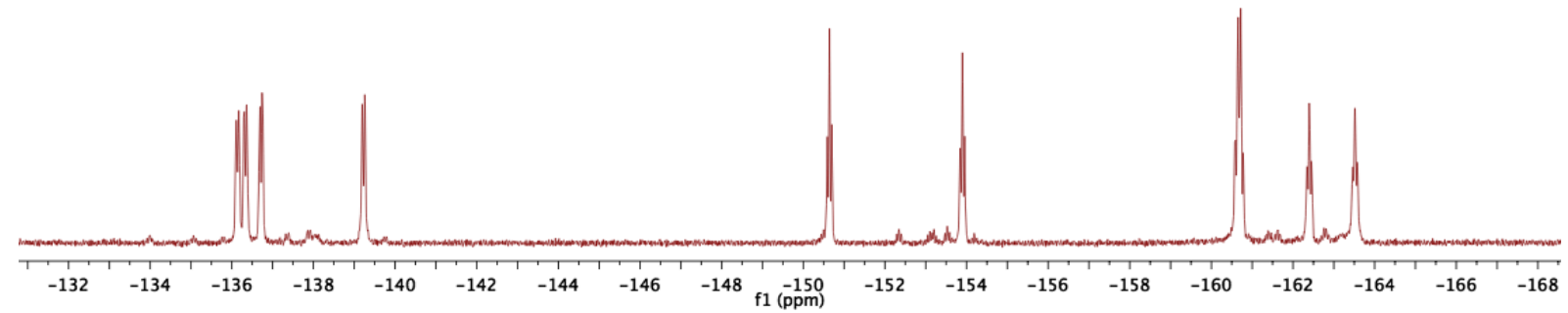


5.5.3 $2 D \operatorname{COSY}\left(\mathrm{CDCl}_{3}, 283 \mathrm{~K}, 500 \mathrm{MHz}\right)$
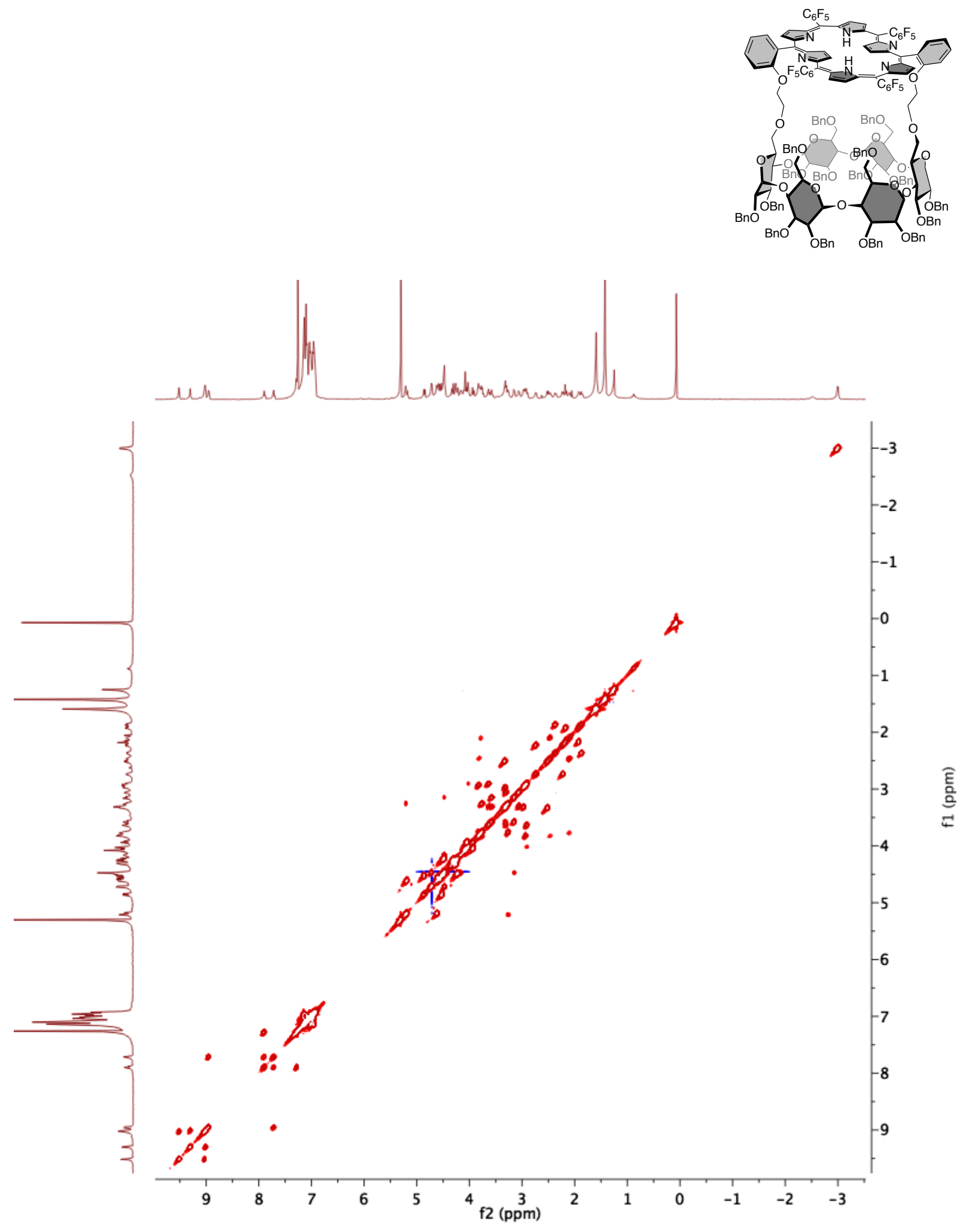
5.5.4 2D TOCSY $\left(\mathrm{CDCl}_{3}, 283 \mathrm{~K}, 500 \mathrm{MHz}, \tau \mathrm{m}=240 \mathrm{~ms}\right)$
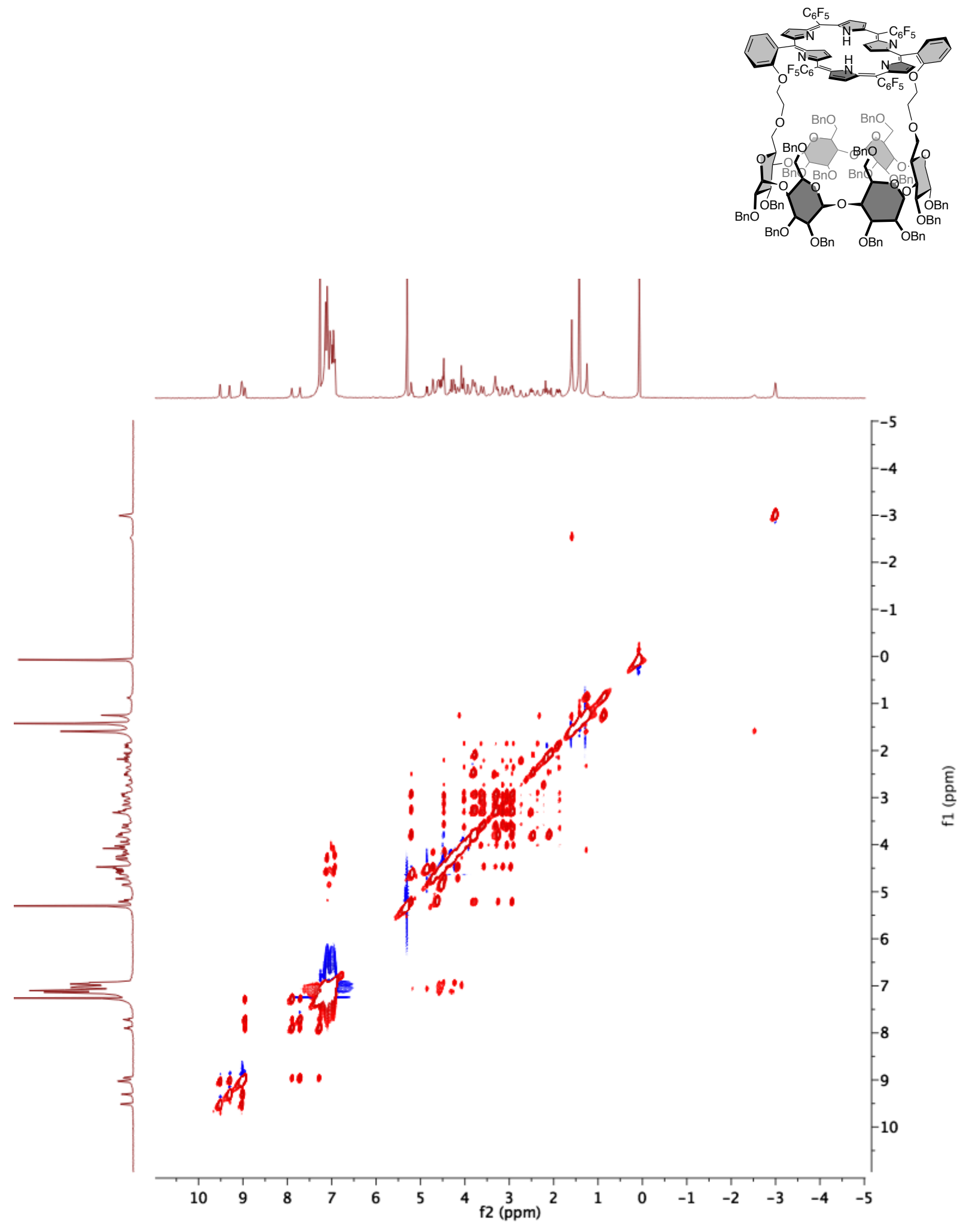
5.5.5 2D HSQC-edited $\left(\mathrm{CDCl}_{3}, 283 \mathrm{~K}, 500 \mathrm{MHz}\right)$
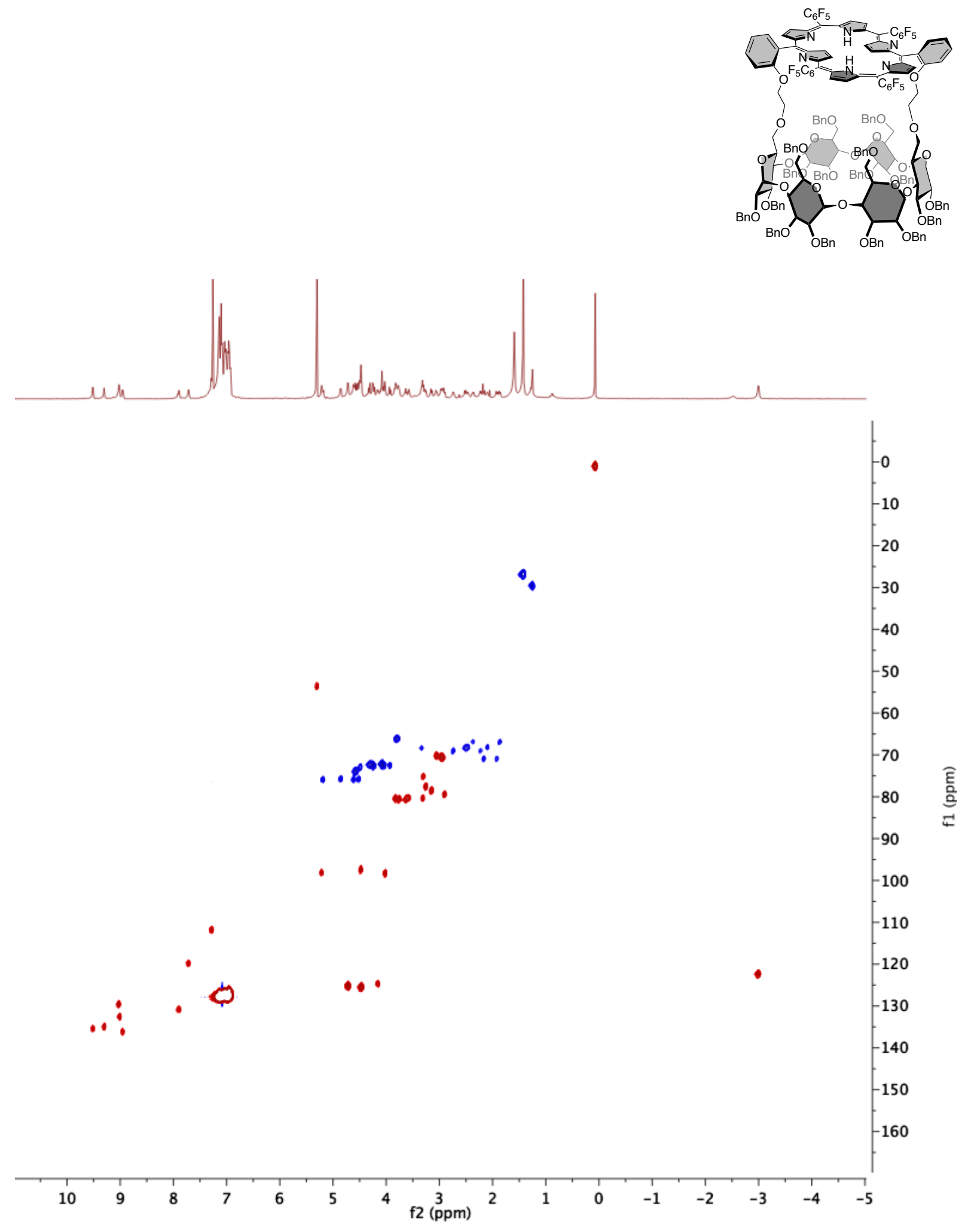
5.5.6 2D NOESY $\left(\mathrm{CDCl}_{3}, 283 \mathrm{~K}, 500 \mathrm{MHz}, \mathrm{\tau m}=800 \mathrm{~ms}\right)$
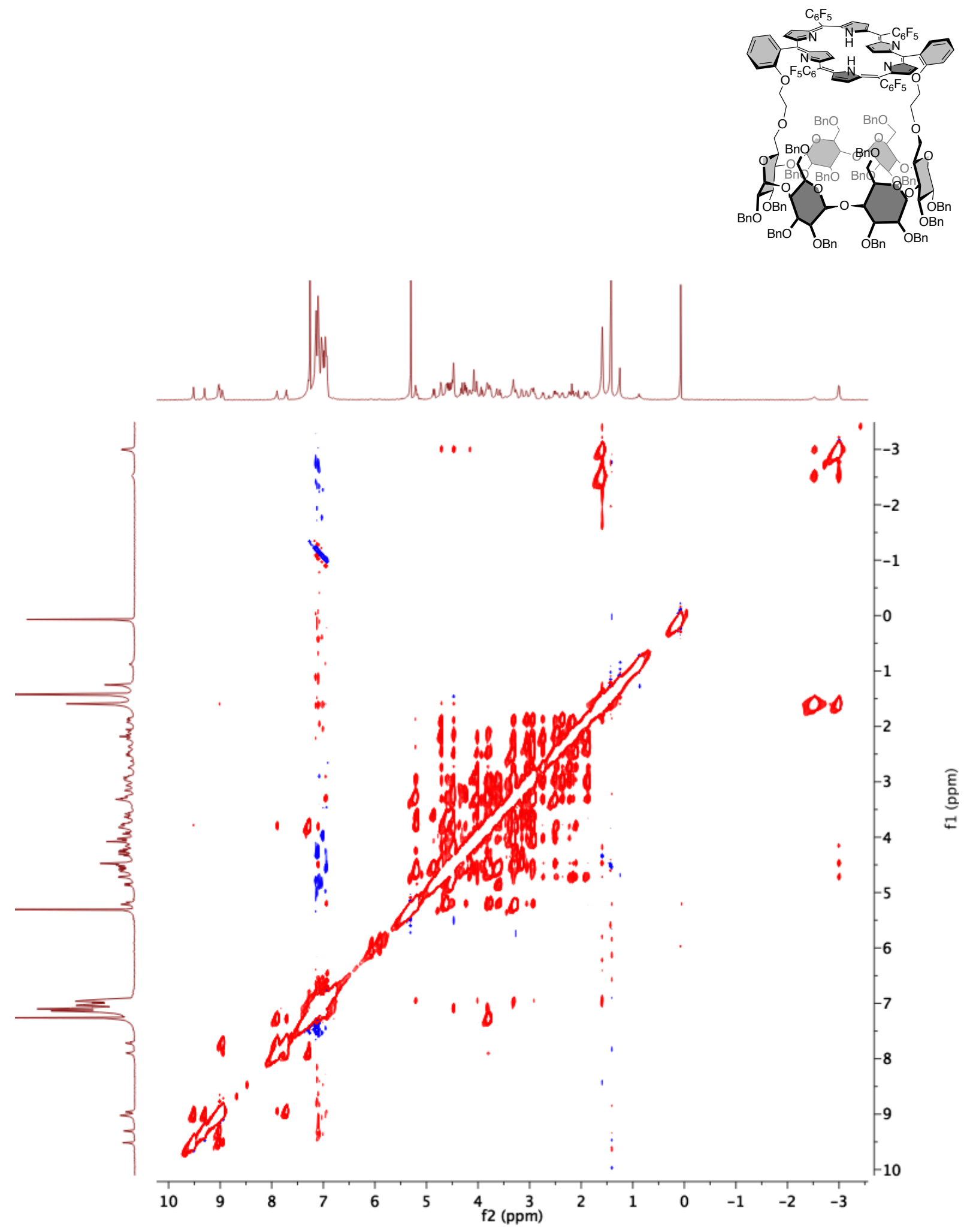
5.5.7 ${ }^{19} \mathrm{~F} 2 \mathrm{D} \operatorname{cosY}\left(\mathrm{CDCl}_{3}, 298 \mathrm{~K}, 376 \mathrm{MHz}\right)$

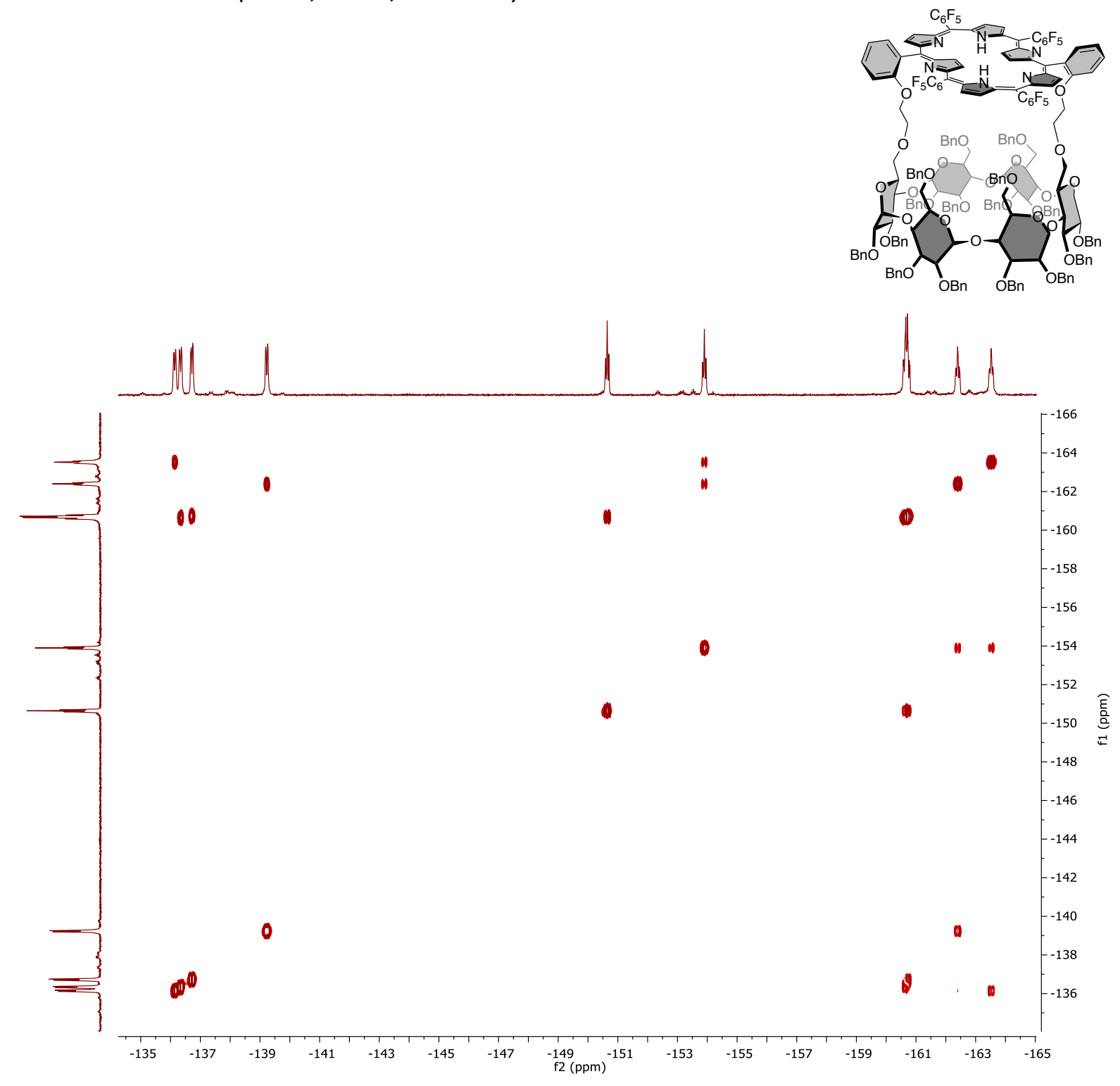




\section{$5.6{ }^{\mathrm{R}}[28] 1$}

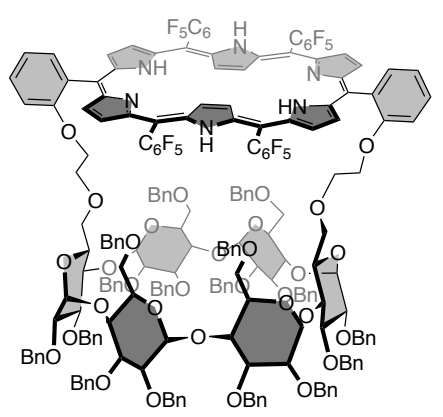

5.6.1 ${ }^{1} \mathrm{H} N M R\left(T H F-d_{8}, 298 K, 500 \mathrm{MHz}\right)$

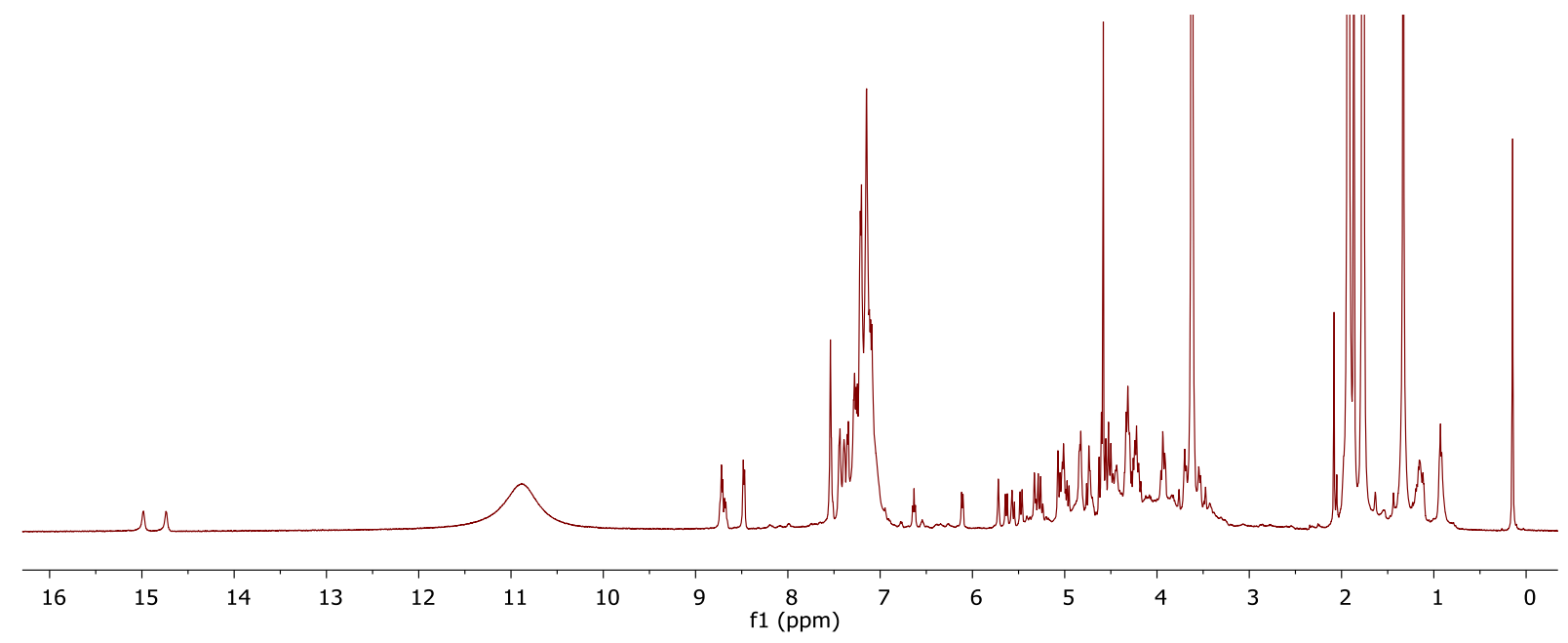

5.6.2 ${ }^{19} \mathrm{FNMR}\left(T H F-d_{8}, 298 \mathrm{~K}, 470 \mathrm{MHz}\right)$

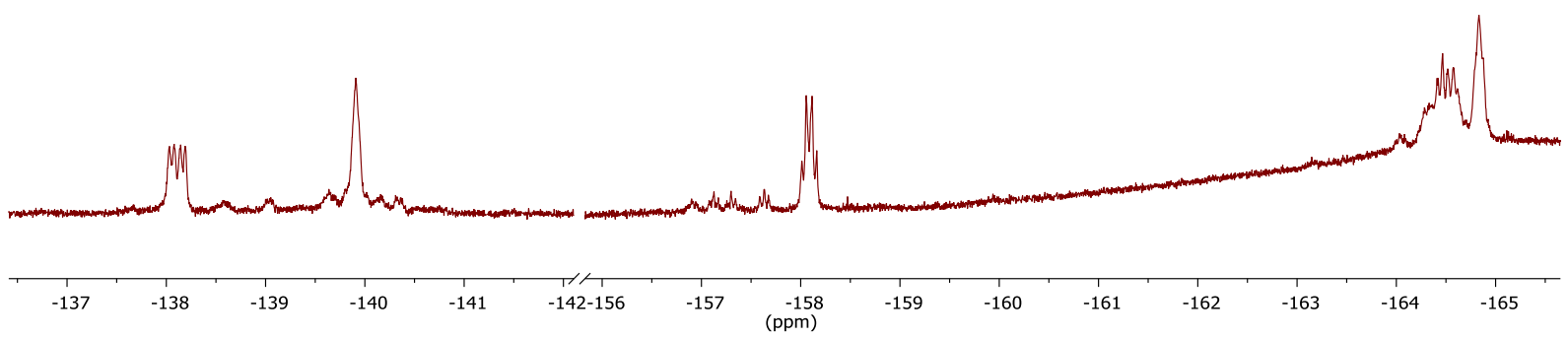


5.6.3 2D COSY $\left(T H F-d_{8}, 273 K, 500 \mathrm{MHz}\right)$

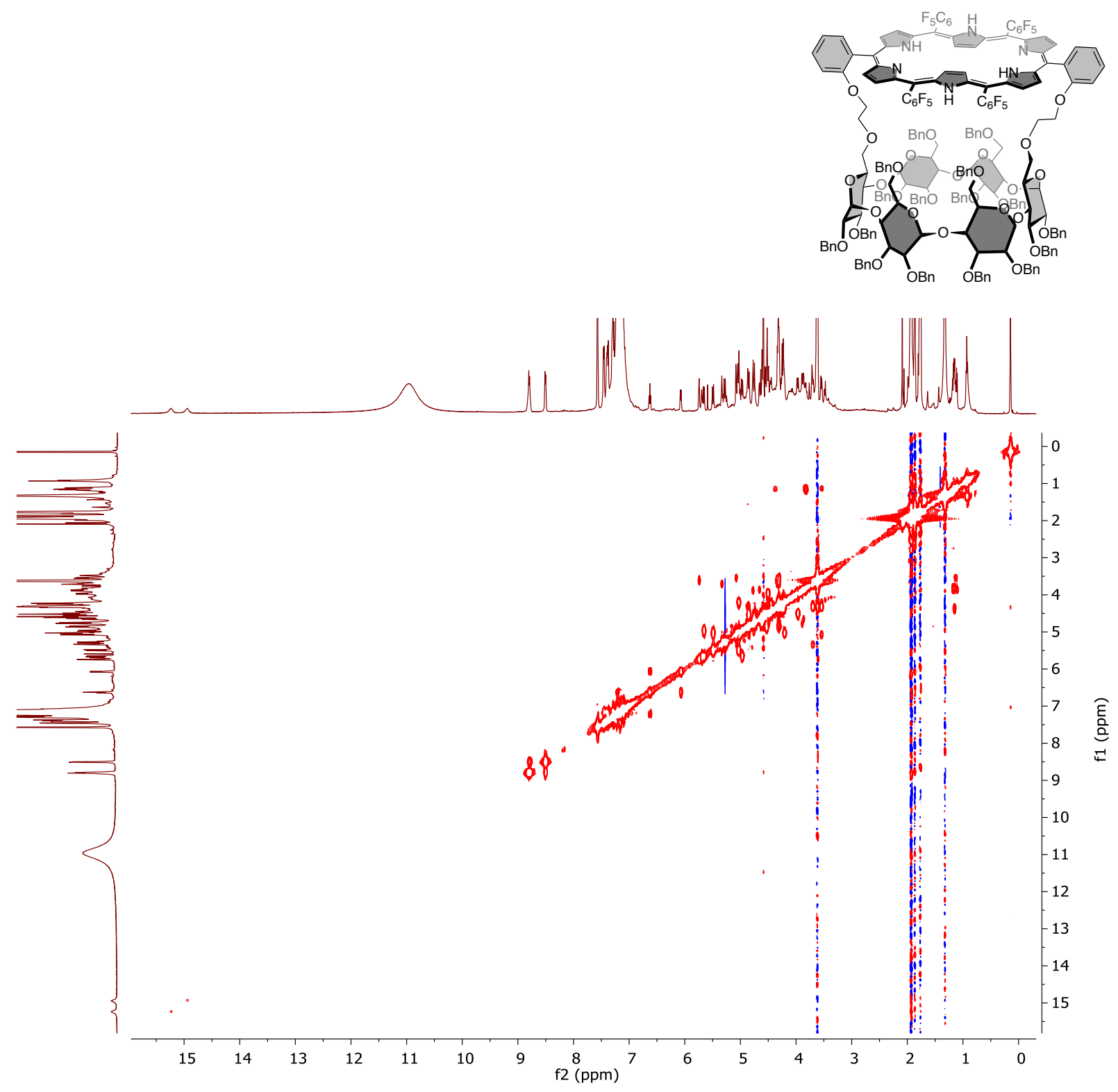


5.6.4 2D HSQC-edited (THF-d $8,298 \mathrm{~K}, 500 \mathrm{MHz}$ )

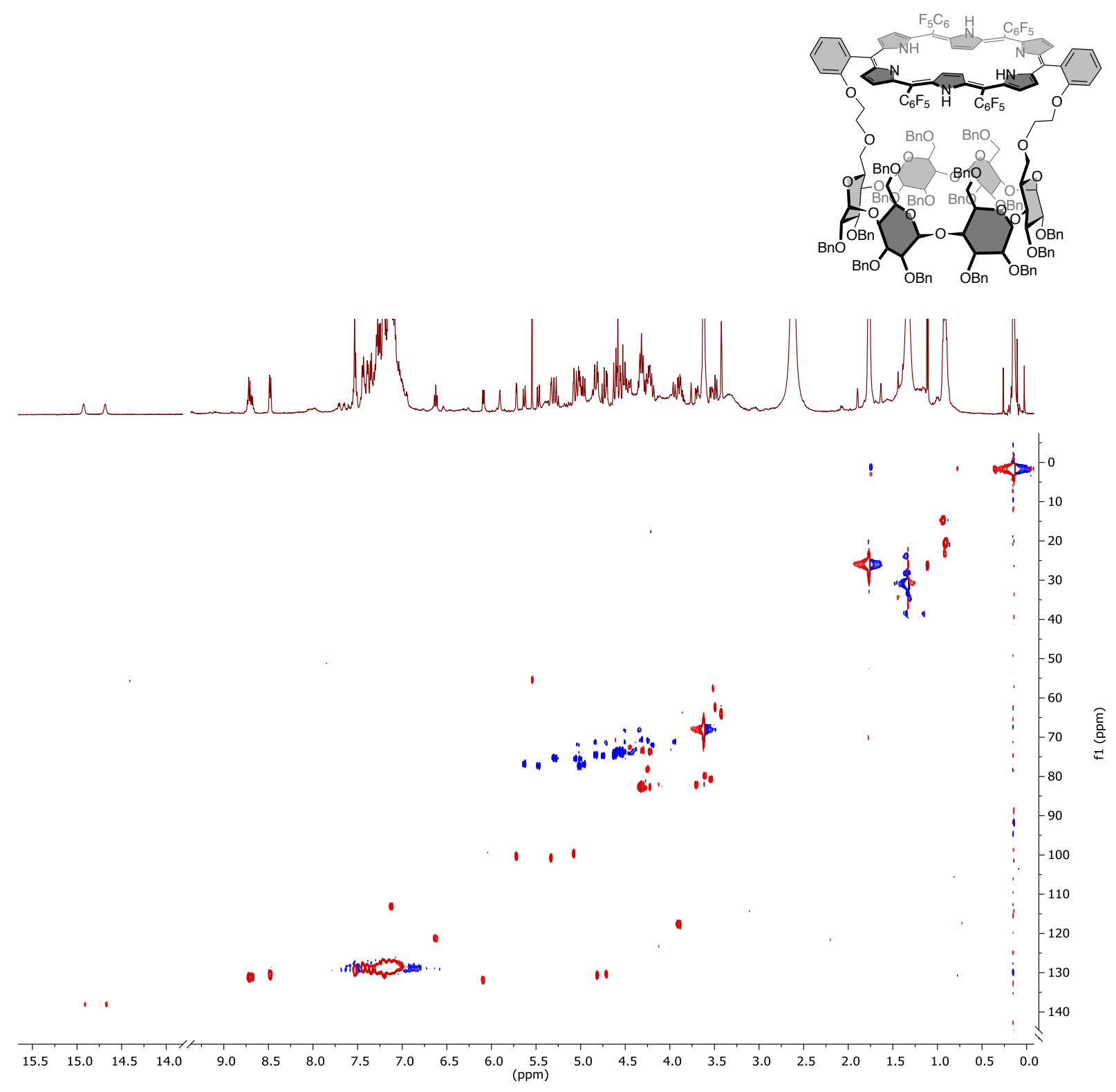


$5.7 \mathrm{M}[28] 2(90: 10$ d.r.)

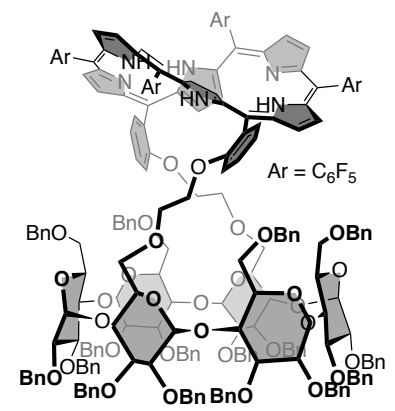

5.7.1 ${ }^{1} \mathrm{HNMR}\left(\mathrm{CDCl}_{3}, 298 \mathrm{~K}, 500 \mathrm{MHz}\right)$

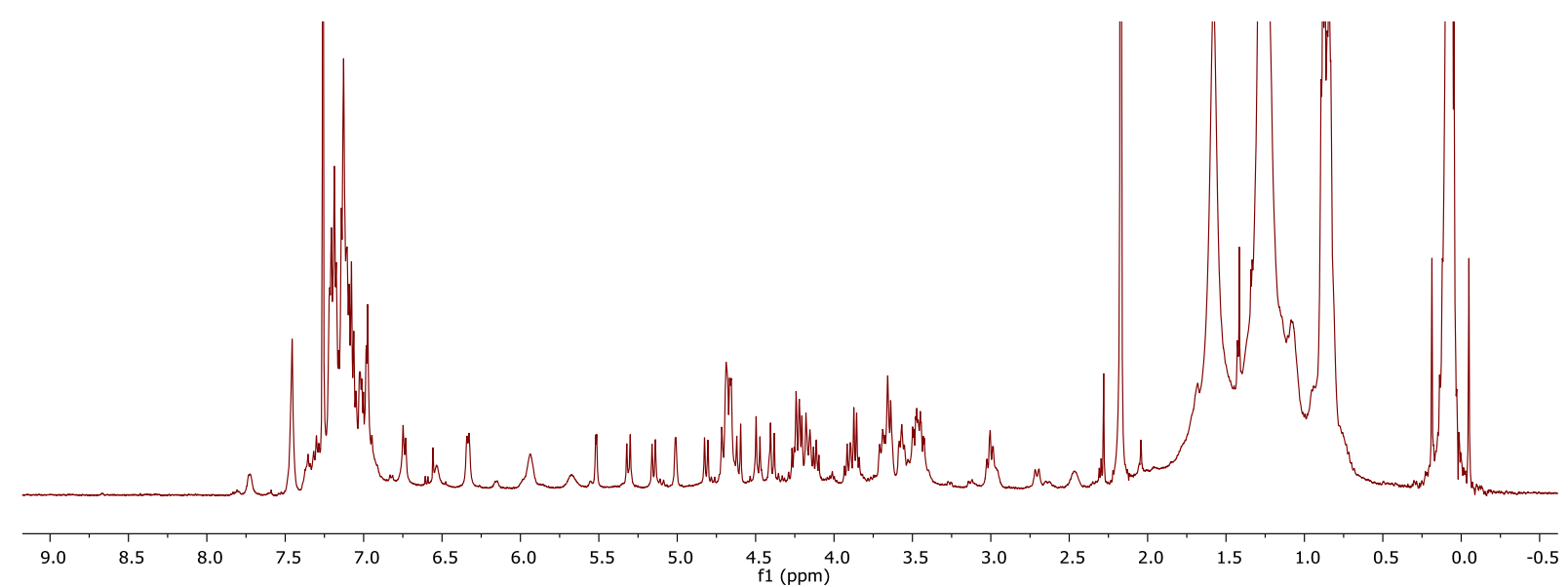




\section{$5.8 \mathrm{M}[28] 3$ (15:85 d.r.)}

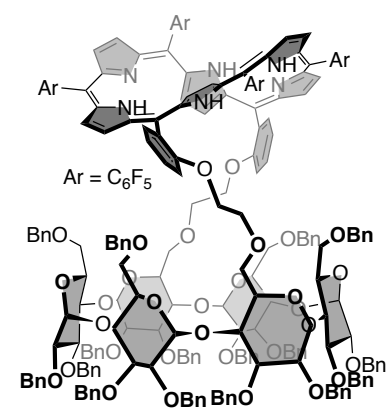

\subsection{1 ${ }^{1} \mathrm{HNMR}\left(\mathrm{CDCl}_{3}, 298 \mathrm{~K}, 500 \mathrm{MHz}\right)$}

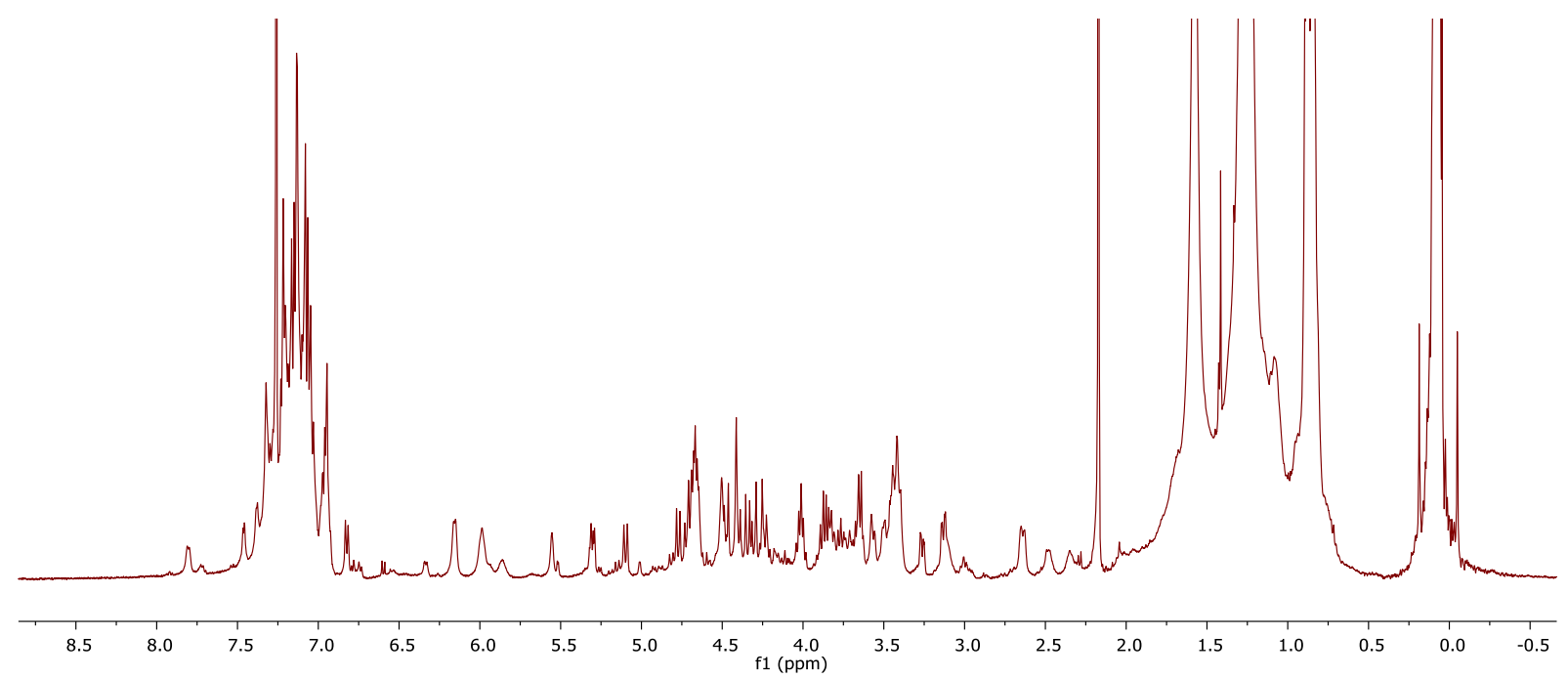




\section{$5.9 \mathrm{M}^{\mathrm{M}}[\mathbf{2 8}] 2 /{ }^{\mathrm{M}}[\mathbf{2 8}] 3$ (55:45 d.r.)}

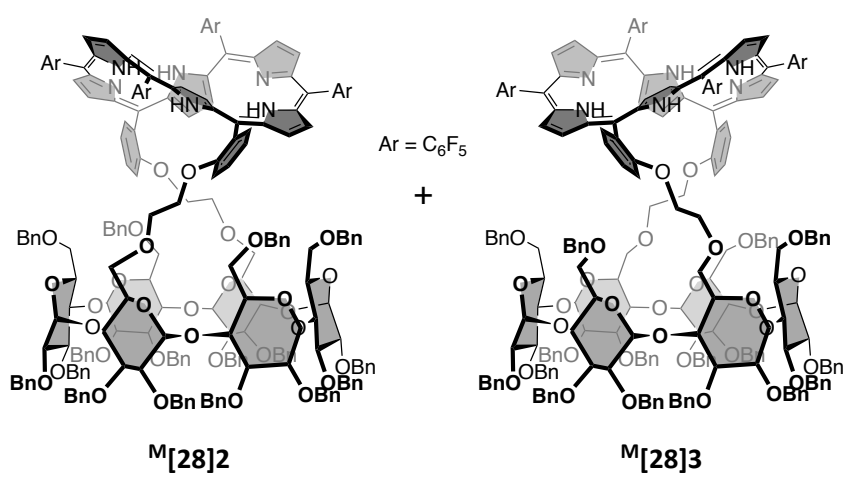

5.9.1 $2 D \operatorname{COSY}\left(\mathrm{CDCl}_{3}, 330 \mathrm{~K}, 500 \mathrm{MHz}\right)$.

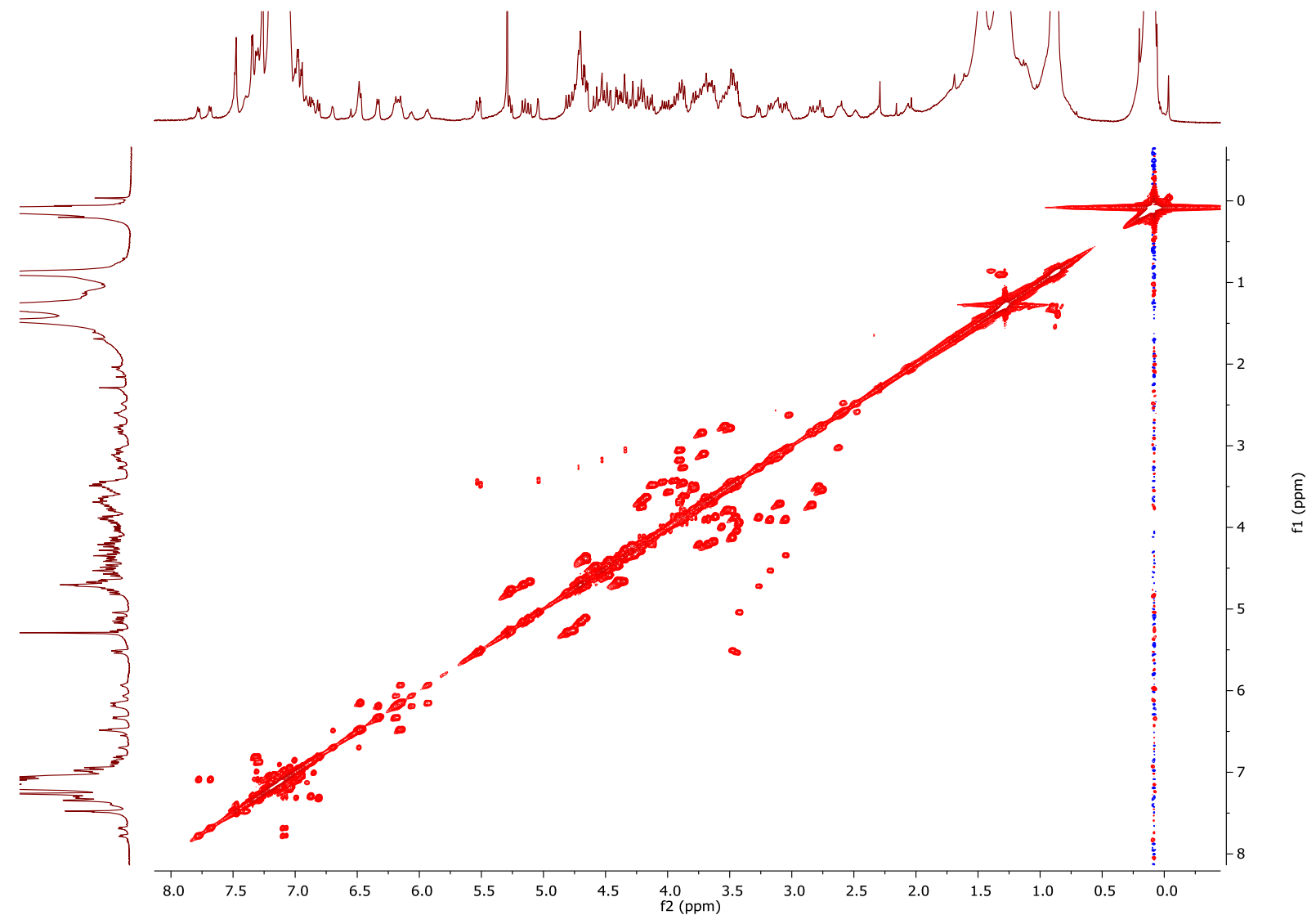


5.9.2 2D TOCSY $\left(\mathrm{CDCl}_{3}, 330 \mathrm{~K}, 500 \mathrm{MHz}\right)$.
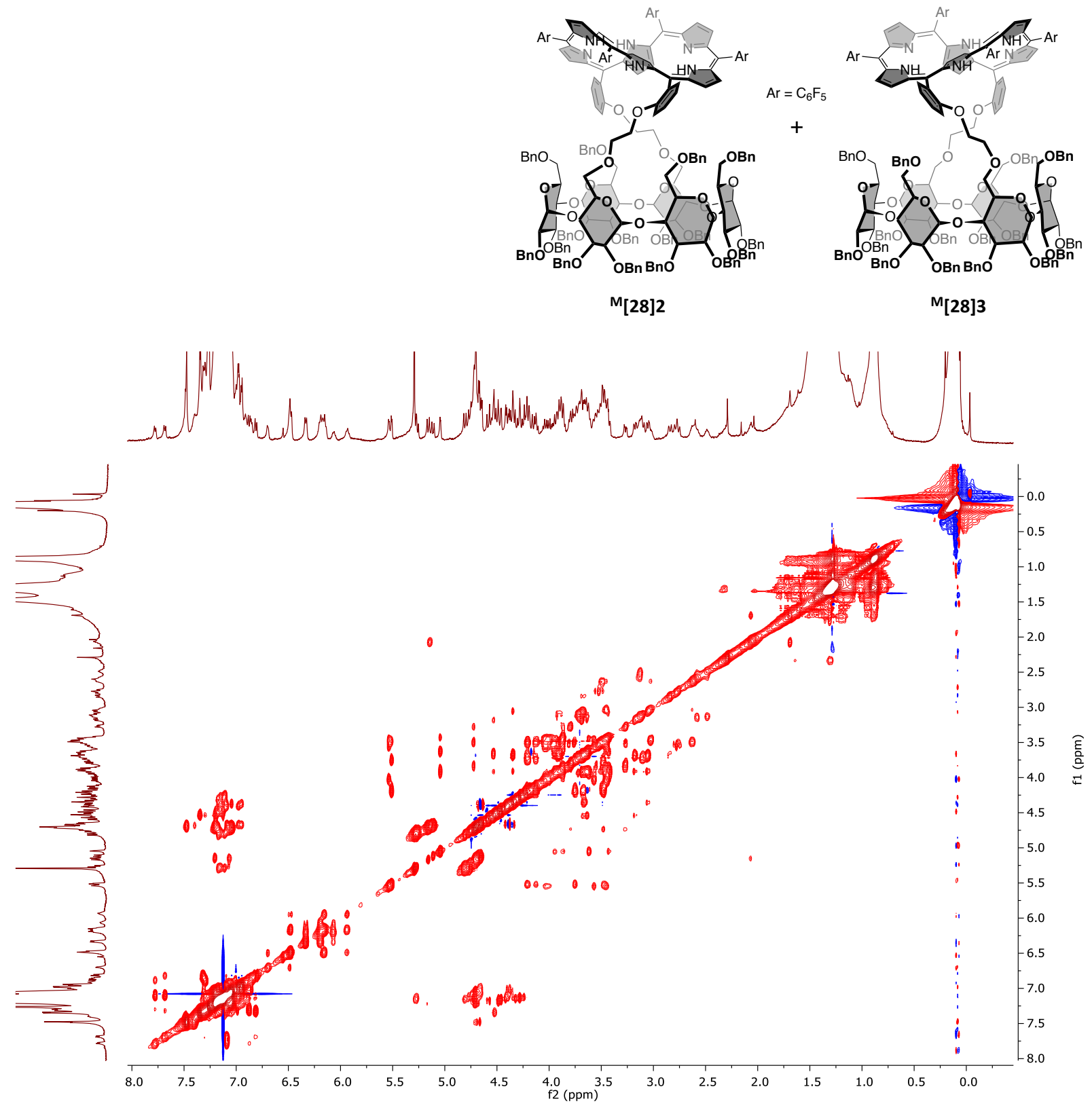
5.9.3 2D HSQC $\left(\mathrm{CDCl}_{3}, 330 \mathrm{~K}, 500 \mathrm{MHz}\right)$.
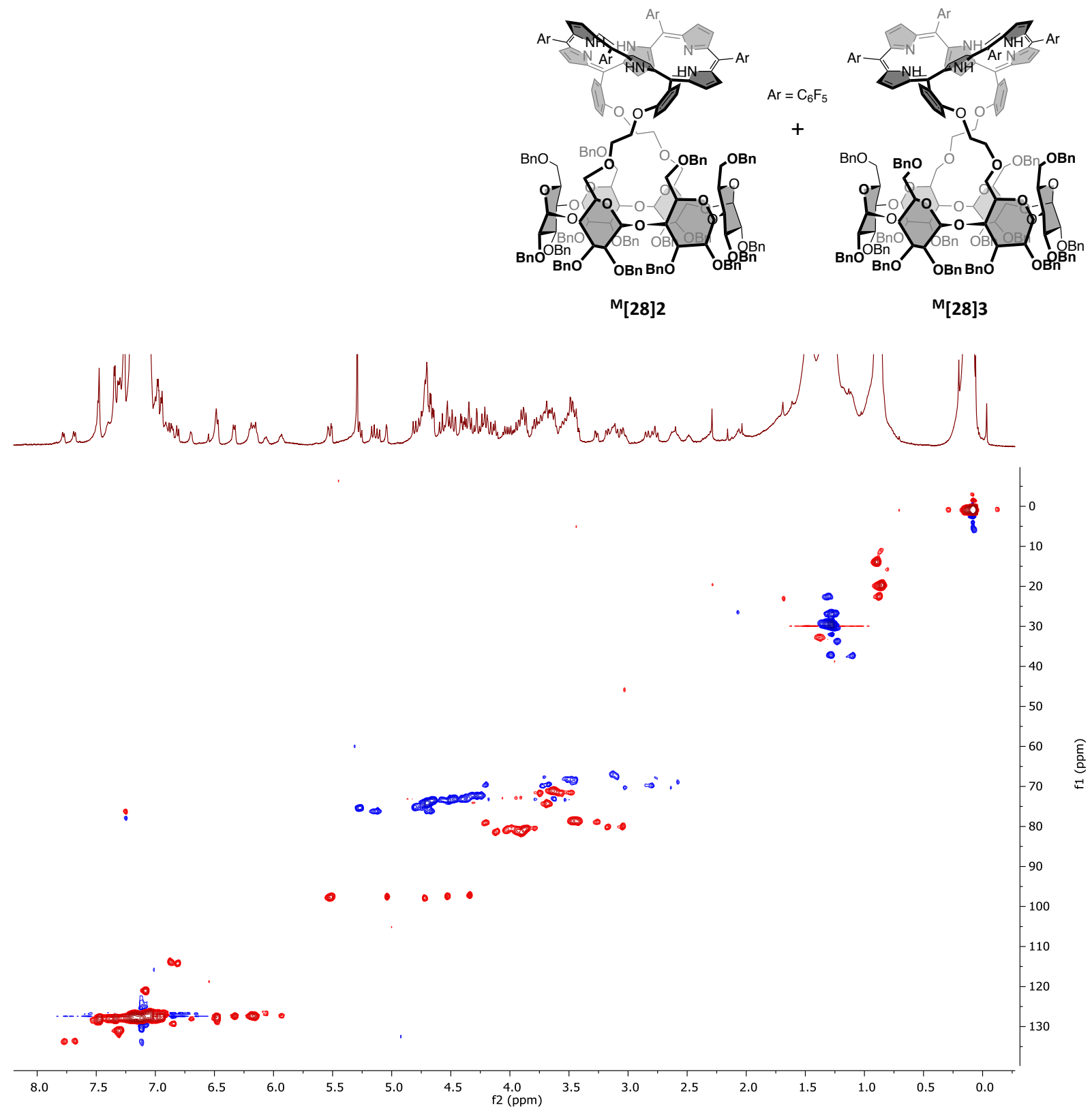
5.9.4 2D NOESY $\left(\mathrm{CDCl}_{3}, 330 \mathrm{~K}, 500 \mathrm{MHz}\right)$.
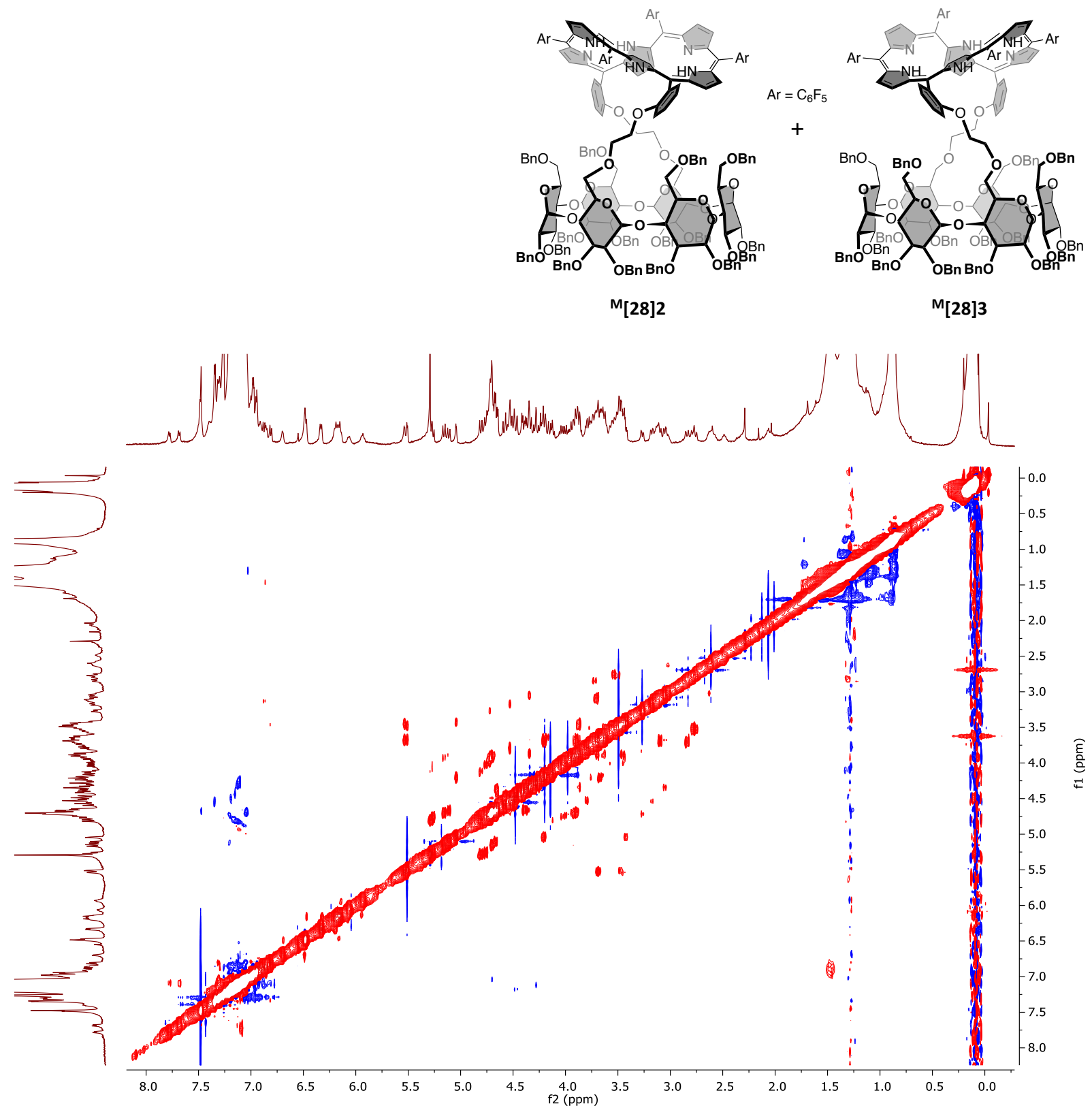
5.9.5 VT ${ }^{1} \mathrm{H} N M R$ spectra $\left(\mathrm{CDCl}_{3}, 330 \mathrm{~K} \rightarrow 223 \mathrm{~K}, 500 \mathrm{MHz}\right)$.
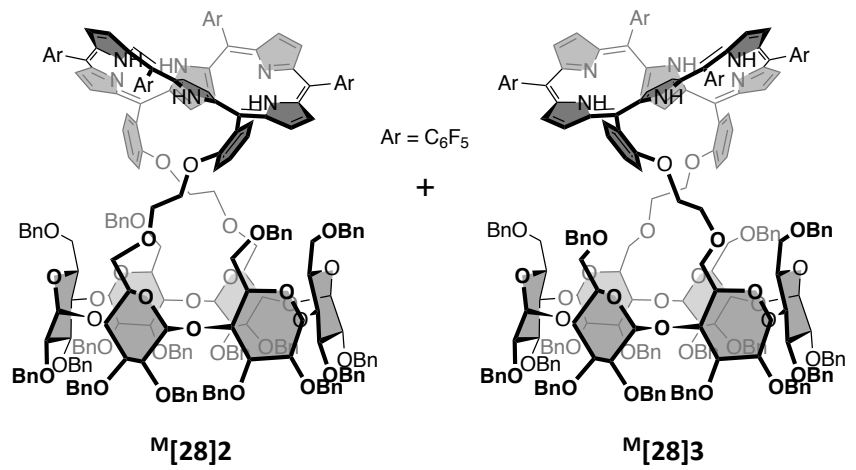

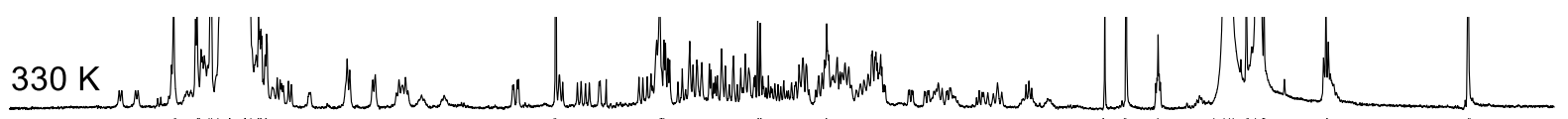

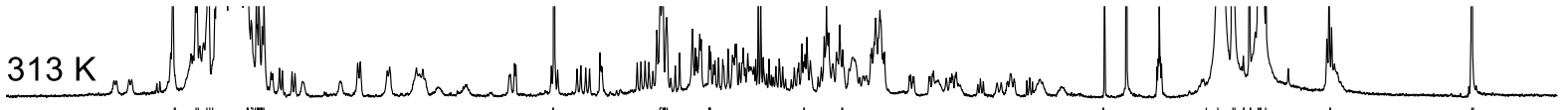

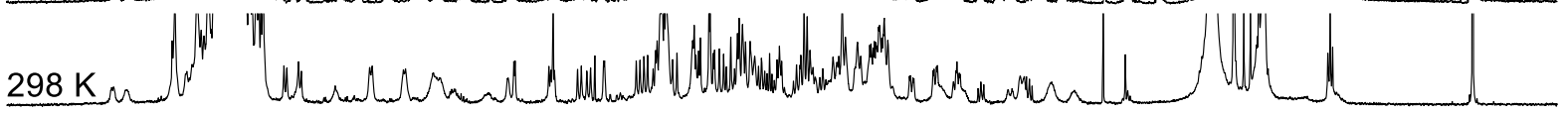

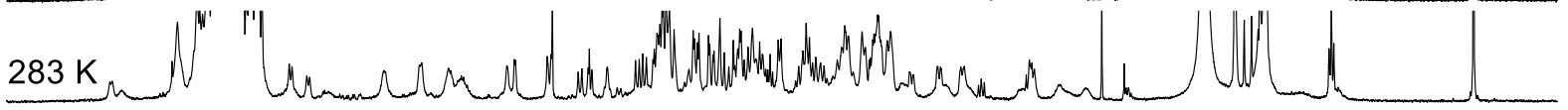

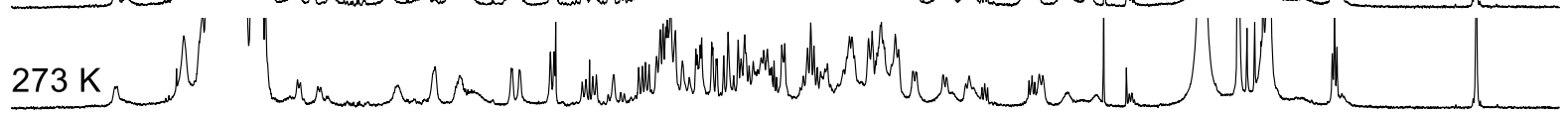

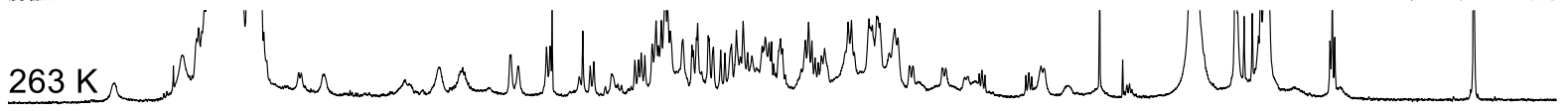

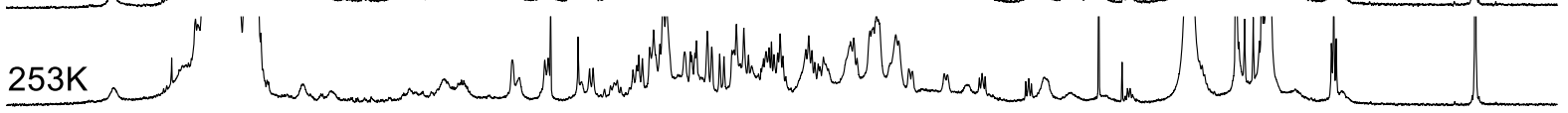

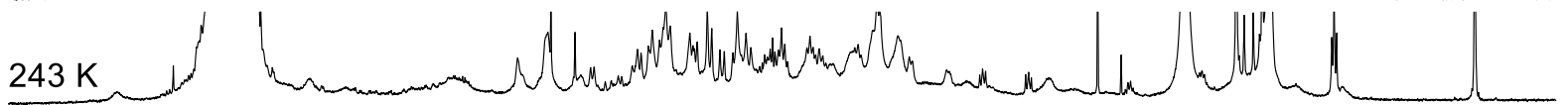

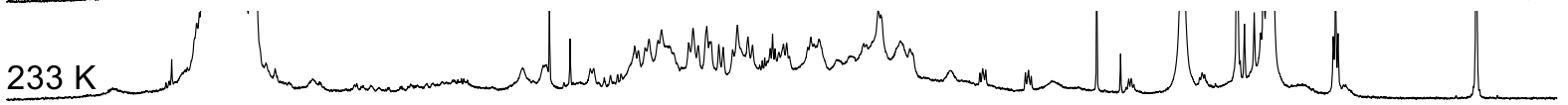

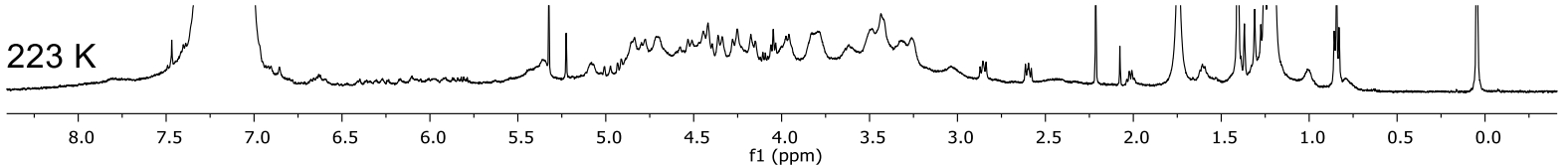




\subsection{Comparison of the ECD spectra in $\mathrm{CH}_{2} \mathrm{Cl}_{2}$ of ${ }^{\mathrm{R}}[26] 1,{ }^{\mathrm{R}}[26] 2,{ }^{\mathrm{R}}[26] 3$, ${ }^{\mathrm{M}}[28] 2$ and ${ }^{\mathrm{M}}[28] 3$}

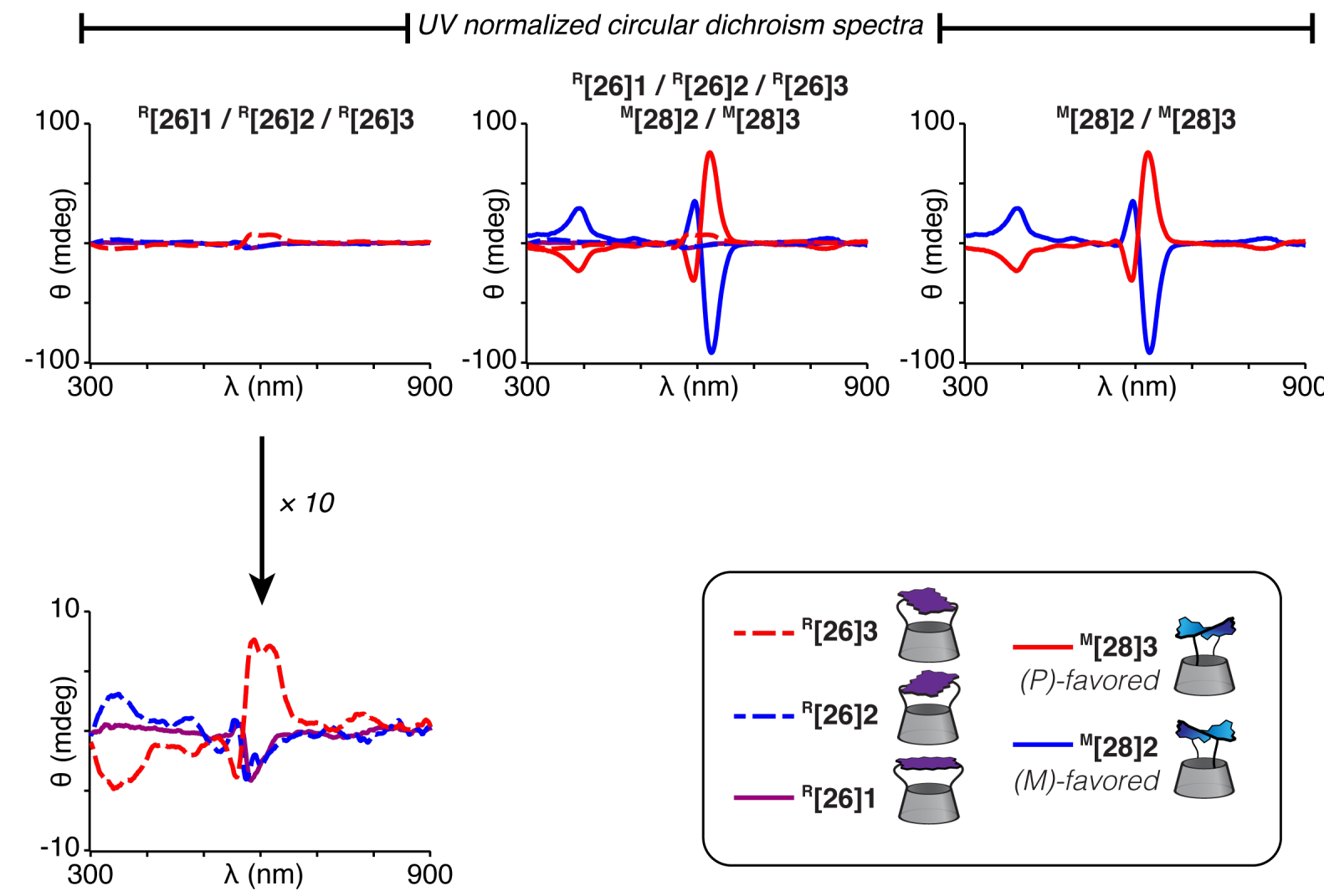

All circular dichroism spectra intensities were scaled according to their corresponding UV-vis absorption spectra normalized at their maximum absorbance. The ${ }^{\mathrm{M}}[\mathbf{2 8}] \mathbf{2}$ and ${ }^{\mathrm{M}}[\mathbf{2 8}] \mathbf{3}$ spectra are enriched mixtures of both components with respectively 90:10 and 15:85 d.r.. 
6 Geometric comparison of the formal Möbius diastereomers of M[28]2 and ${ }^{\mathrm{M}}$ [28]3

6.1 Reassignment of the absolute configuration of the [28]hexaphyrin palladium complex 2A (X-ray structure taken from Osuka et al. Angew. Chem. Int. Ed. 2010, 49, 6619; CCDC 767696), exhibiting negative Cotton effect.

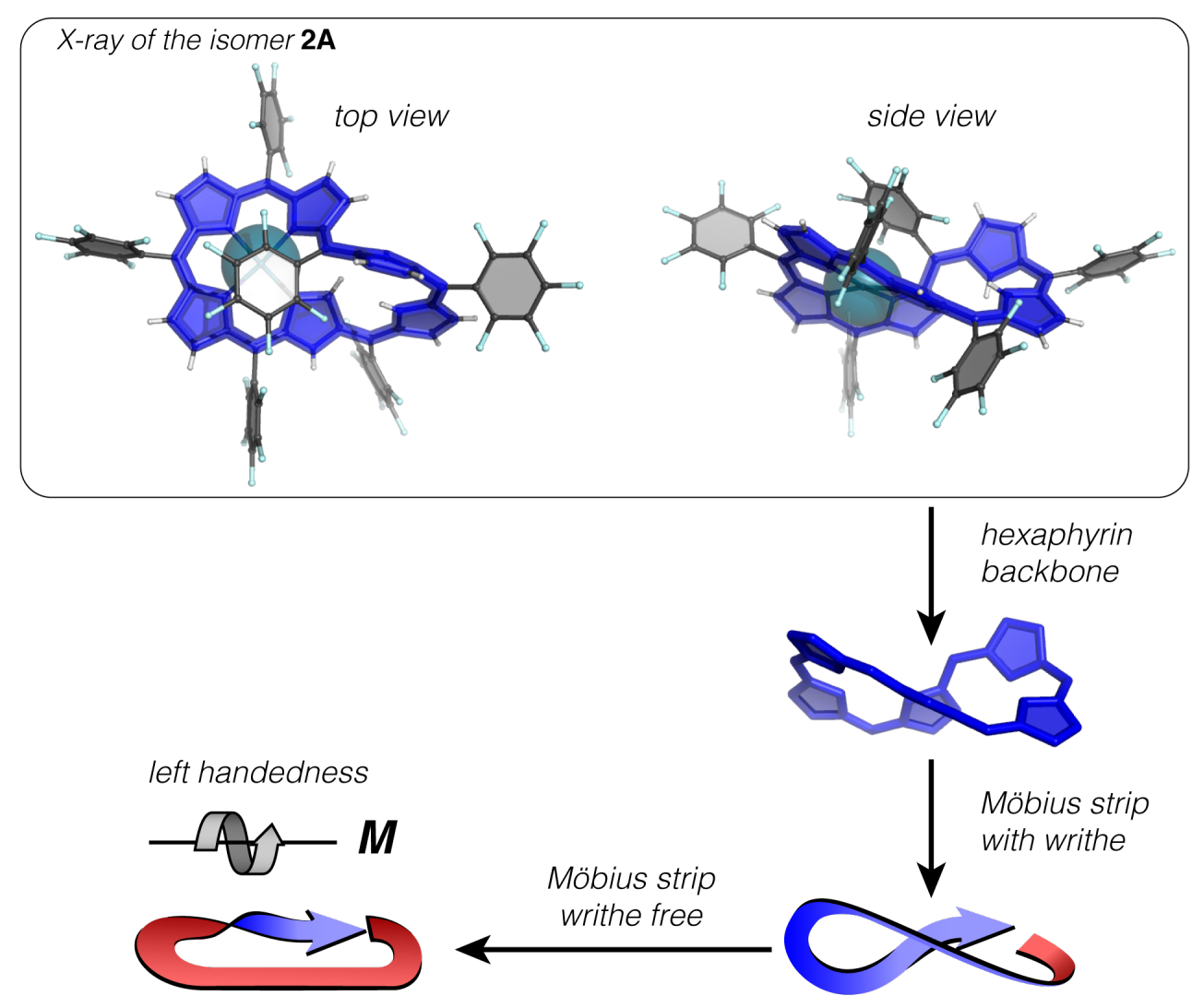

See the following reference for a definition of the $M / P$ stereodescriptors: Stępień, M.; Sprutta, N.; Latos-Grażyński, L. Angew. Chem., Int. Ed. 2011, 50, 4288-4340 (pp 4300-4301, Scheme 6). 


\subsection{Molecular models of formal Möbius diastereomers of ${ }^{\mathrm{M}}[\mathbf{2 8}] \mathbf{2}$ and $\mathrm{M}[28] 3$}

Molecular models (geometry optimization with Avogadro, UFF force field, benzyl groups omitted) of the $M$ and $P$ twist Möbius configurations for both ${ }^{\mathrm{M}}[28] 2$ and ${ }^{\mathrm{M}}[28] 3$ considering the planar chirality of the substitution pattern $\left(R_{\mathrm{p}} v s . S_{\mathrm{p}}\right)$ and the twisted pyrrole isomerism $\left(\beta \pi^{\mathrm{up}} v s . \beta \pi^{\text {down }}\right)$. The meso- $\mathrm{C}_{6} \mathrm{~F}_{5}$ substituent responsible for the selectivity is highlighted in red.

Stereogenic elements

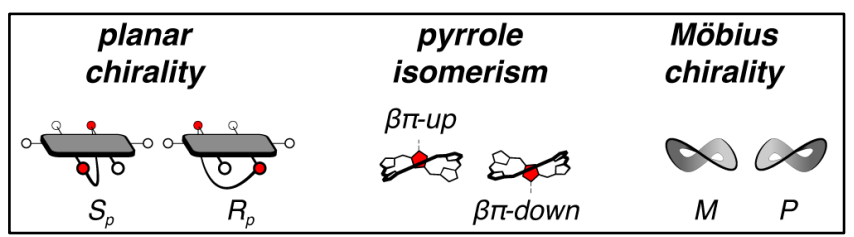

\section{$R_{\mathrm{p}}{ }^{\mathrm{M}}[28] 2$}

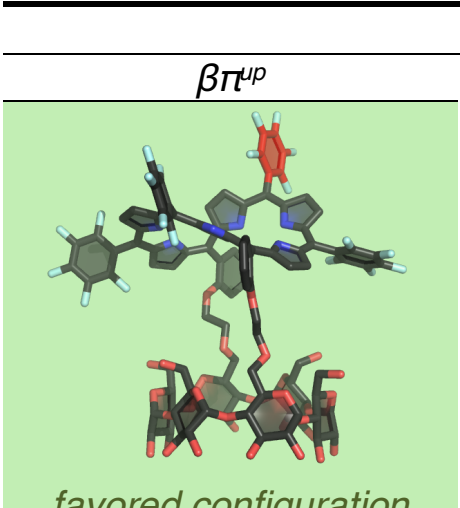

favored configuration

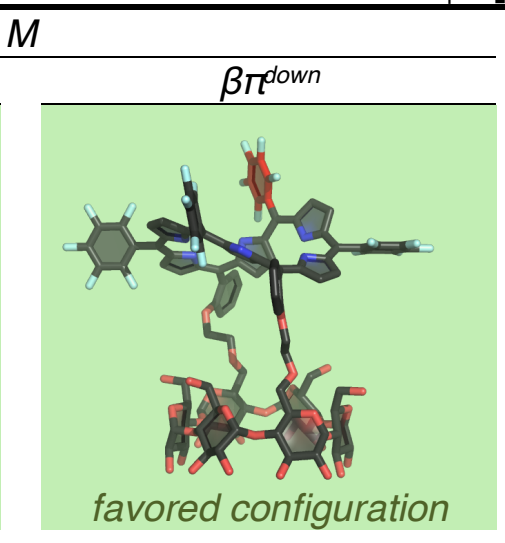

\begin{tabular}{cc}
$P$ \\
\hline$\beta \pi^{u p}$ & $\beta \pi^{\text {down }}$ \\
\hline
\end{tabular}
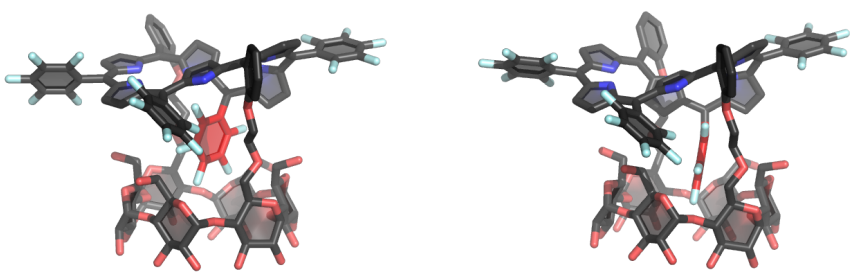

$S_{\mathrm{p}-\mathrm{M}[28] 3}$

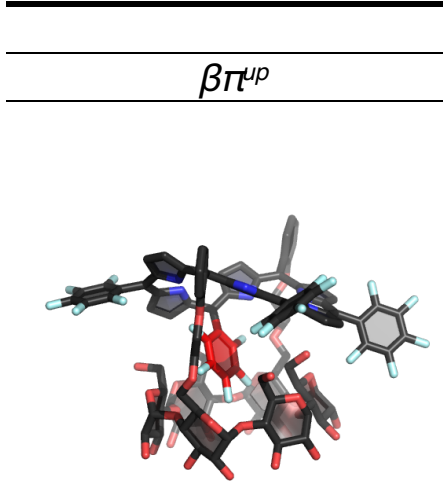

\section{M}

$\beta \pi^{\text {down }}$

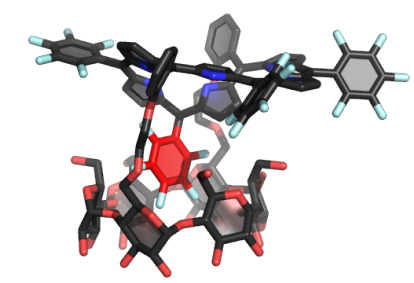

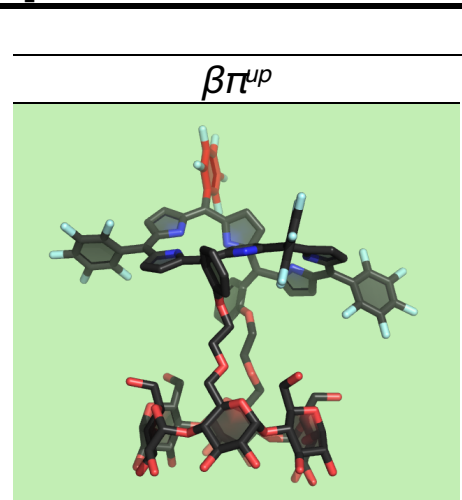

favored configuration
$\mathrm{P}$

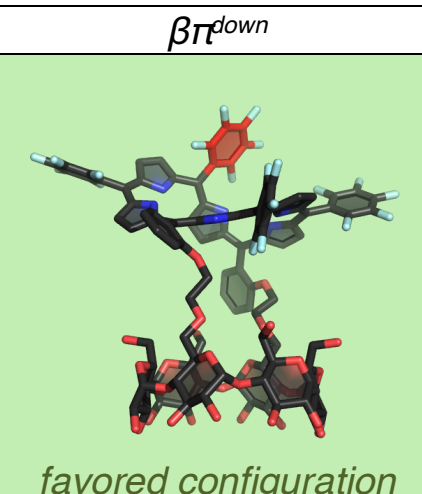




\section{Metalation}

\subsection{Metalation of ${ }^{\mathrm{M}}[\mathbf{2 8}] \mathbf{2} /{ }^{\mathrm{M}}[\mathbf{2 8}] 3$ with $\mathrm{Zn}(\mathrm{OAc})_{2}$}

\subsubsection{Procedure}

In a standard NMR tube, $3 \mathrm{mg}(0.80 \mu \mathrm{mol})$ of a $1: 1$ mixture of ${ }^{\mathrm{M}}[\mathbf{2 8}] \mathbf{2} /{ }^{\mathrm{M}}[\mathbf{2 8}] 3$ were dissolved in $500 \mu \mathrm{L}$ of 9:1 $\mathrm{CDCl}_{3} / \mathrm{CD}_{3} \mathrm{OD}$. $120 \mu \mathrm{L}$ of a $40 \mathrm{mM}$ solution of $\mathrm{Zn}(\mathrm{OAc})_{2}$ in 9:1 $\mathrm{CDCl}_{3} / \mathrm{CD}_{3} \mathrm{OD}$ (4.80 $\mu \mathrm{mol}, 6$ equiv)

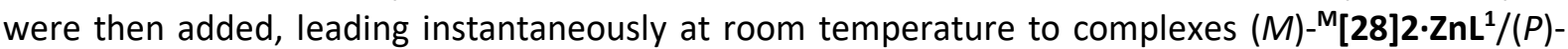
${ }^{\mathrm{M}}[28] 3 \cdot \mathrm{ZnL}^{1}$ (partial metallation, see text).

\subsection{2 ${ }^{1} H$ NMR titration and subsequent evolution}

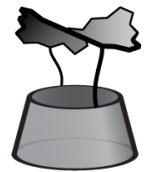

${ }^{\mathrm{M}}[28] 2$

d.r. $90: 10$
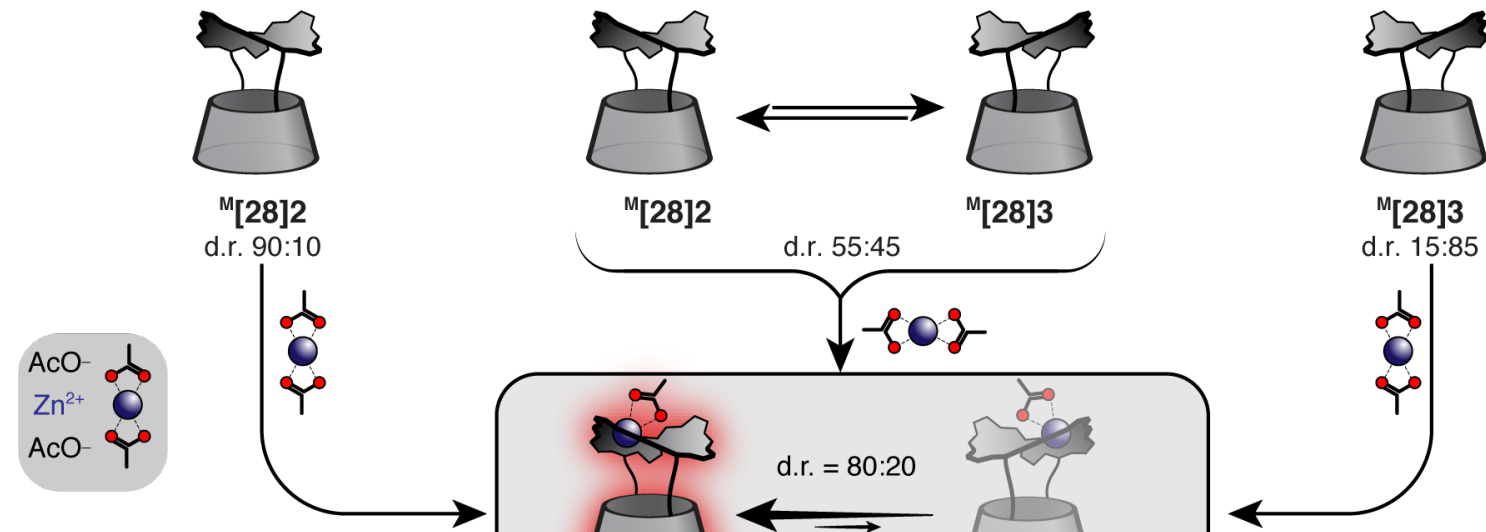

${ }^{\mathrm{M}}[28] 2$

${ }^{\mathrm{M}}[28] 3$

M[28]3

d.r. $15: 85$ .r. 55:45

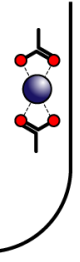

(M) ${ }^{\mathrm{M}}[28] 2 \cdot \mathrm{Zn}^{\mathrm{OA}}$

- $(P))^{\mathrm{M}}[28] 3 \cdot \mathrm{Zn}^{\mathrm{OAC}}$
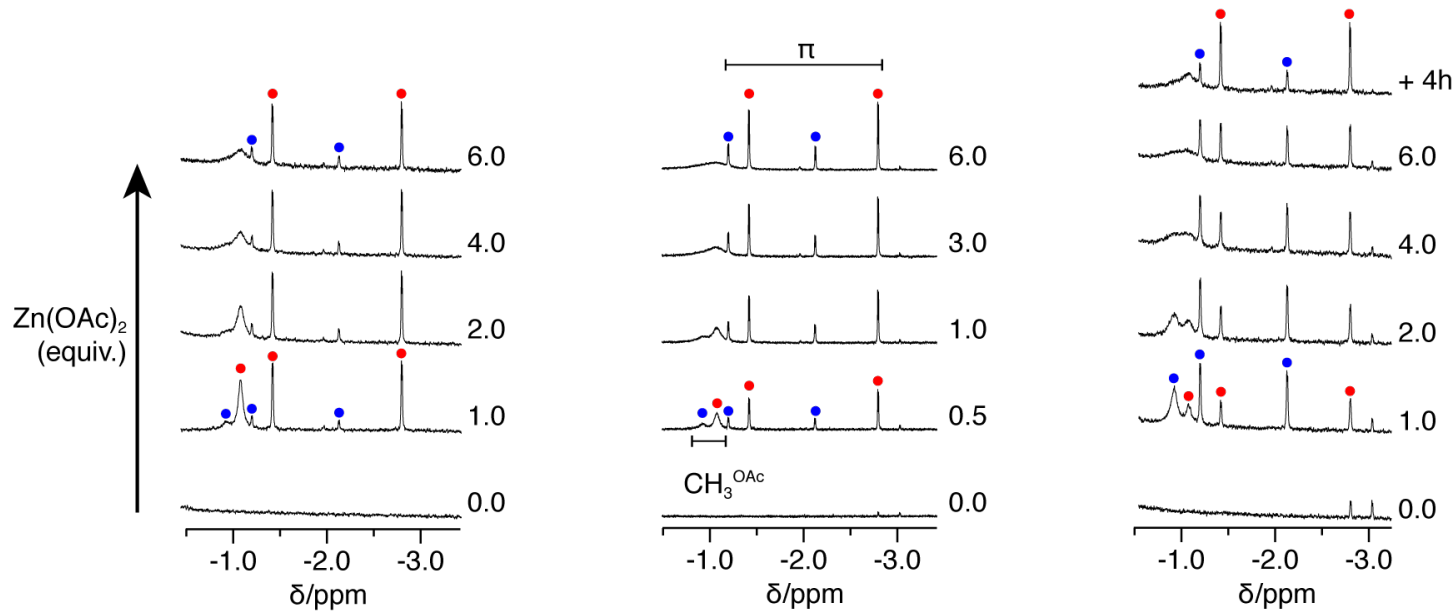
7.1.3 VT ${ }^{1} H$ NMR of (M)- $\left.{ }^{M}[28] 2 \cdot \mathrm{ZnL}^{1} /(P)\right)^{M}[28] 3 \cdot \mathrm{ZnL}^{1}$ and $2 D$ HSQC and NOESY NMR spectra $\left(C D \mathrm{Cl}_{3} / C D_{3} \mathrm{OD}, 9: 1,260 \mathrm{~K}\right.$ selected regions)

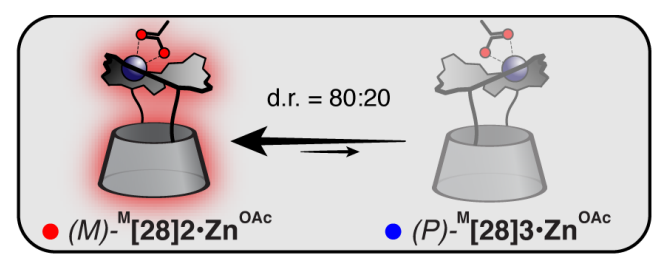

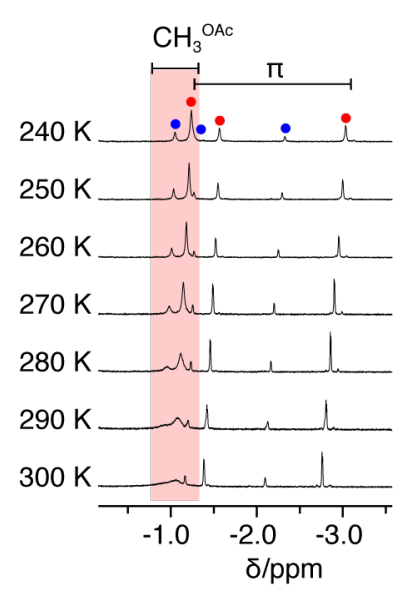

VT-NMR

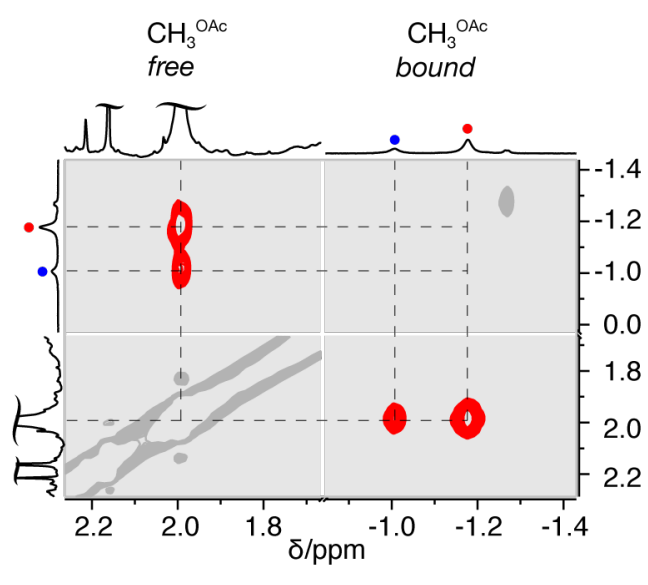

EXSY

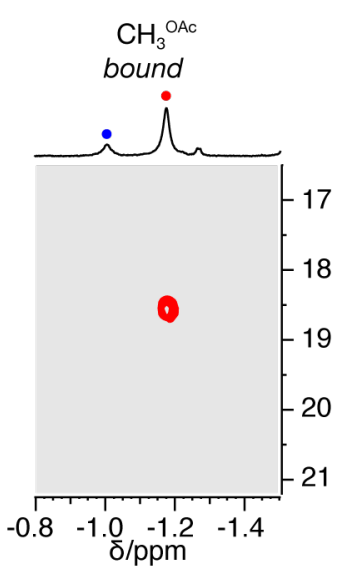

HSQC

\subsubsection{ECD spectra after evolution}

pathway $A$

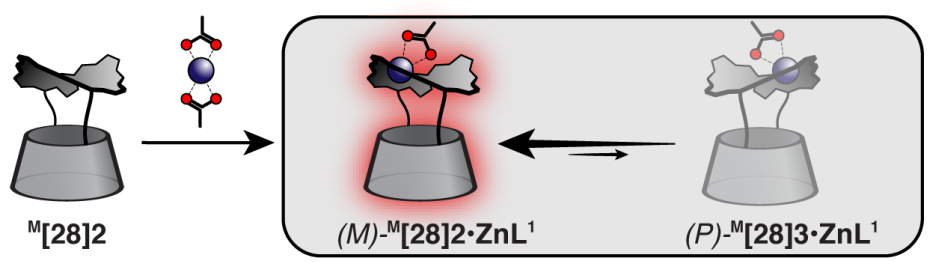

pathway $A$

NMR tube (t+24h), d.r.: 90:10

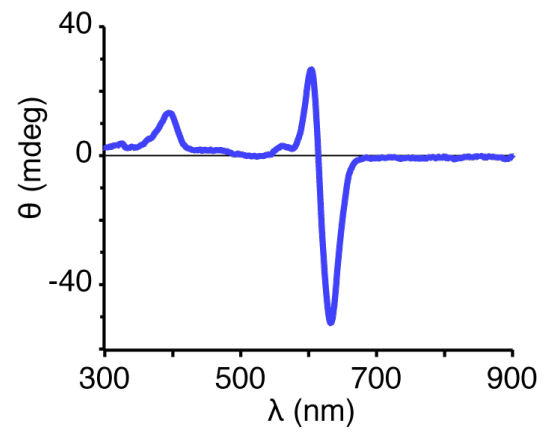

pathway $B$

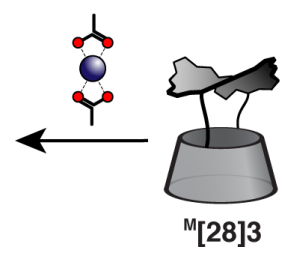

pathway $B$

NMR tube (t+24h), d.r.: 85:15

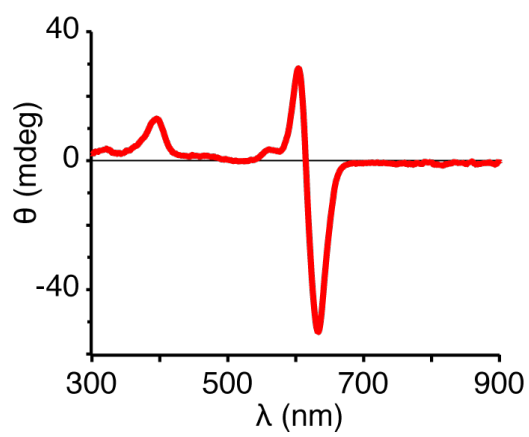

ECD spectra $\left(\mathrm{CHCl}_{3} / \mathrm{CH}_{3} \mathrm{OH}\right.$ 9:1) of the ${ }^{1} \mathrm{H}$ NMR titration of ${ }^{\mathrm{M}}$ [28]2 (pathway $A$ ) or ${ }^{\mathrm{M}}$ [28]3 (pathway B) with $\mathrm{Zn}(\mathrm{OAc})_{2}$ (see part 7.1.2) after $24 \mathrm{~h}$ evolution. 
7.1.5 DDQ oxidation of (M)-M $[28] 2 \cdot \mathrm{ZnL}^{1} /(P)-{ }^{M}[28] 3 \cdot \mathrm{ZnL}^{1}$ to ${ }^{R}[26] 2 /{ }^{R}[26] 3$
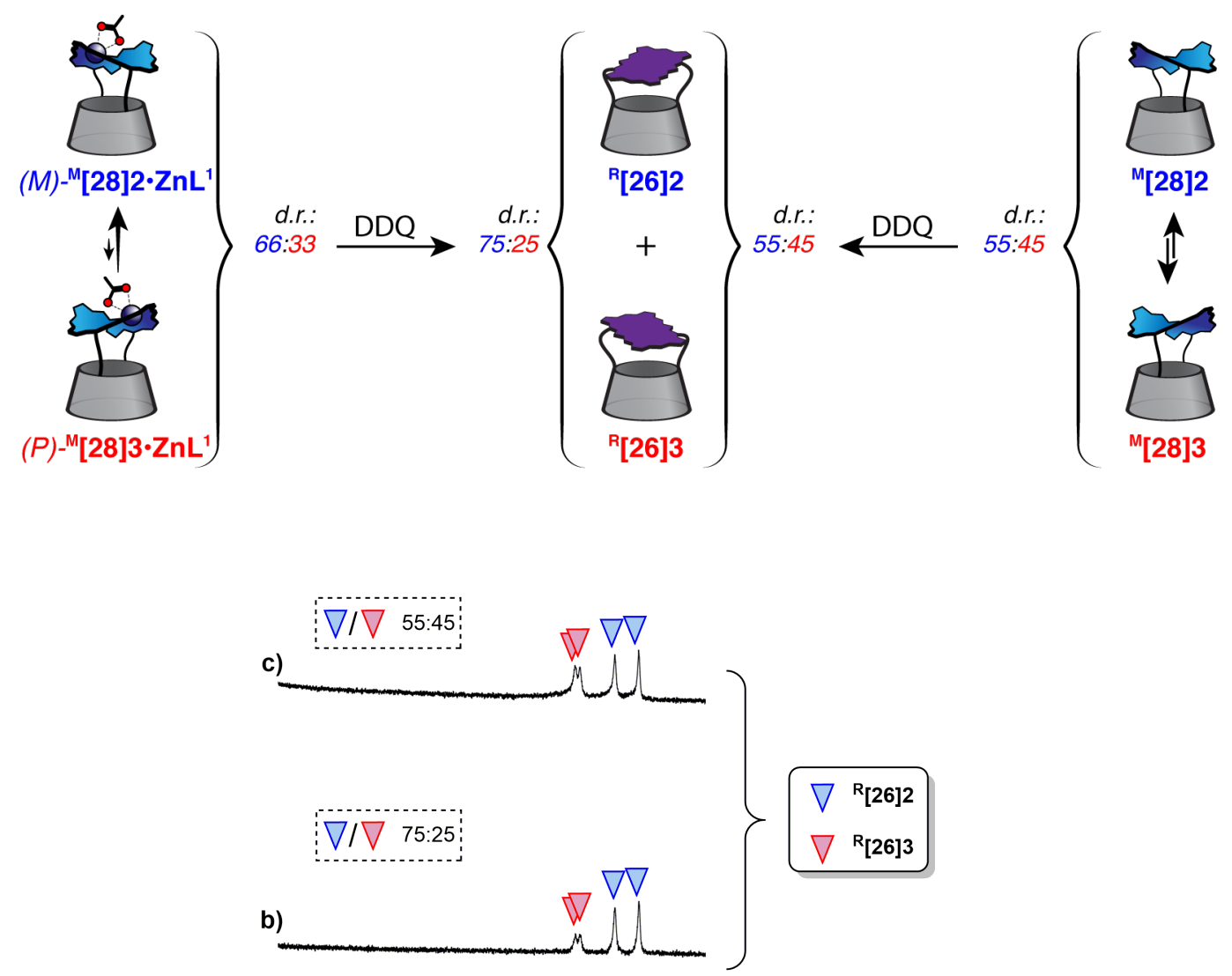

\section{$0 / 066: 33$}

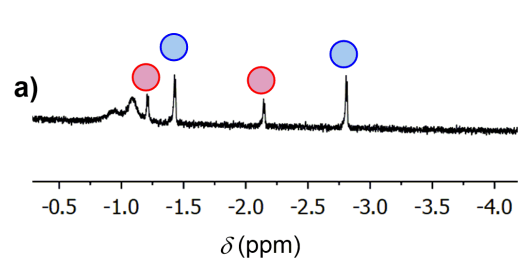

$(M)-{ }^{\mathrm{M}}[28] 2 . \mathrm{ZnL}^{1}$
$(P)-{ }^{\mathrm{M}}[28] 3 . \mathrm{ZnL}^{1}$

${ }^{1} \mathrm{H}$ NMR spectra of complexes $\left.(M){ }^{\mathrm{M}}[\mathbf{2 8}] 2 \cdot \mathrm{ZnL}^{1} /(P)\right)^{\mathrm{M}}[28] 3 \cdot \mathrm{ZnL}^{1}$ ( 66:33 d.r., partial metallation) before (a) and after (b) oxidation with DDQ. (c) ${ }^{1} \mathrm{H} N M R$ spectra obtained after oxidation with DDQ of the 55:45 mixture of ${ }^{\mathrm{M}}[\mathbf{2 8}] 2 /{ }^{\mathrm{M}}$ [28]3. Conditions: $\mathrm{CDCl}_{3} / \mathrm{CD}_{3} \mathrm{OD}$ 9:1, $298 \mathrm{~K}$, selected region (inner $\beta$-pyrrolic protons). 


\subsection{Metallation of ${ }^{\mathrm{M}}[\mathbf{2 8}] 2 /{ }^{\mathrm{M}}[28] 3$ with $\mathrm{Zn}(\mathrm{OTf})_{2} / \mathrm{L}^{2-6}$}

\subsubsection{General procedure}

In a standard NMR tube, $3 \mathrm{mg}(0.80 \mu \mathrm{mol})$ of a $1: 1$ mixture of ${ }^{\mathrm{M}}[\mathbf{2 8}] 2 /{ }^{\mathrm{M}}[28] 3$ were dissolved in $500 \mu \mathrm{L}$ of 9:1 $\mathrm{CDCl}_{3} / \mathrm{CD}_{3} \mathrm{OD} .1 \mu \mathrm{L}$ (5.74 $\mu \mathrm{mol}, 7$ equiv) of DIPEA, $40 \mu \mathrm{L}$ of a $40 \mathrm{mM}$ solution of $\mathrm{Zn}(\mathrm{OTf})_{2}$ in 9:1 $\mathrm{CDCl}_{3} / \mathrm{CD}_{3} \mathrm{OD}$ (1.60 $\mu \mathrm{mol}, 2$ equiv) and $1 \mu \mathrm{L}$ ( $10-12$ equiv) of acac derivative $L^{n}$ were subsequently added. The NMR tube solution was heated $1 \mathrm{~h}$ at $60{ }^{\circ} \mathrm{C}$, affording quasi-quantitatively complexes $(M)$ ${ }^{\mathrm{M}}[\mathbf{2 8}] 2 \cdot \mathrm{ZnL}^{\mathrm{n}} /(P)-^{\mathrm{M}}[\mathbf{2 8}] 3 \cdot \mathrm{ZnL}^{\mathrm{n}}$ (with $\mathrm{n}=2,3,4,6$; partial metallation with $n=5$, see text).

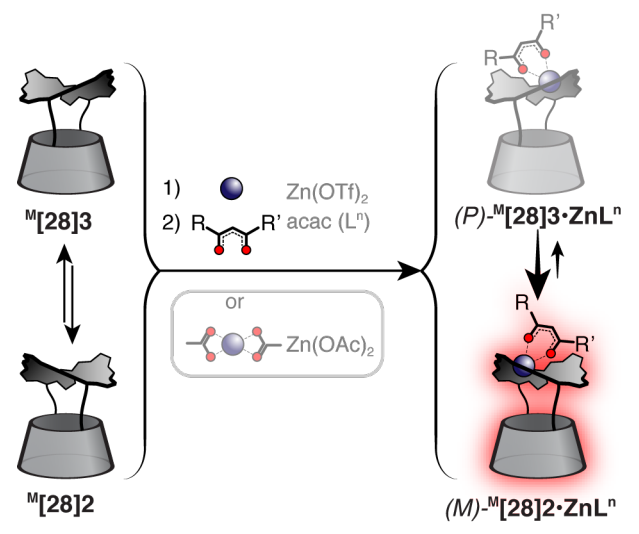

7.2.2 Comparison of the down (observed) and up (putative) orientation of the twisted pyrrole for (M)-M[28]2·ZnL ${ }^{2}$

a)
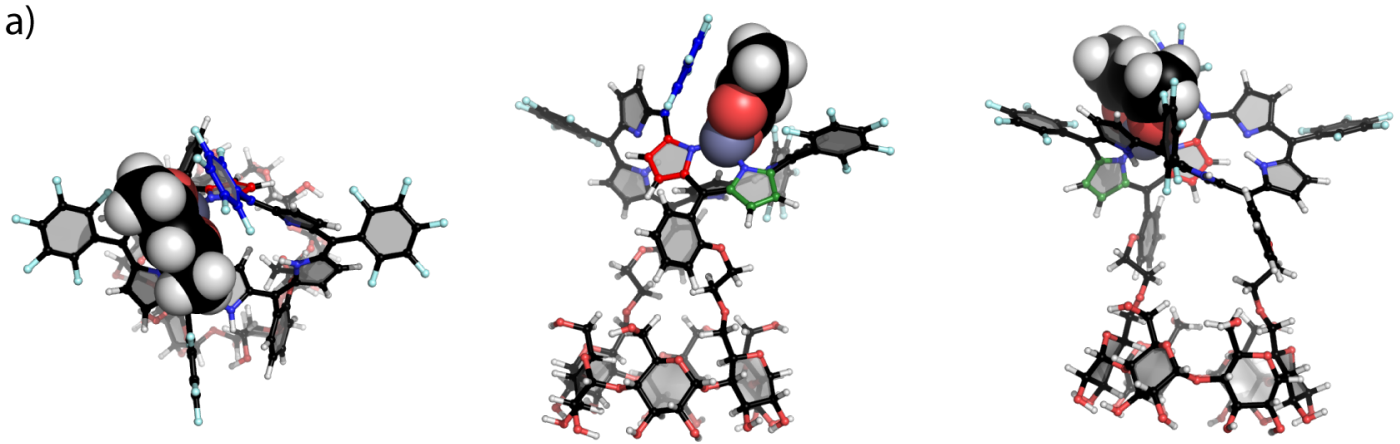

b)
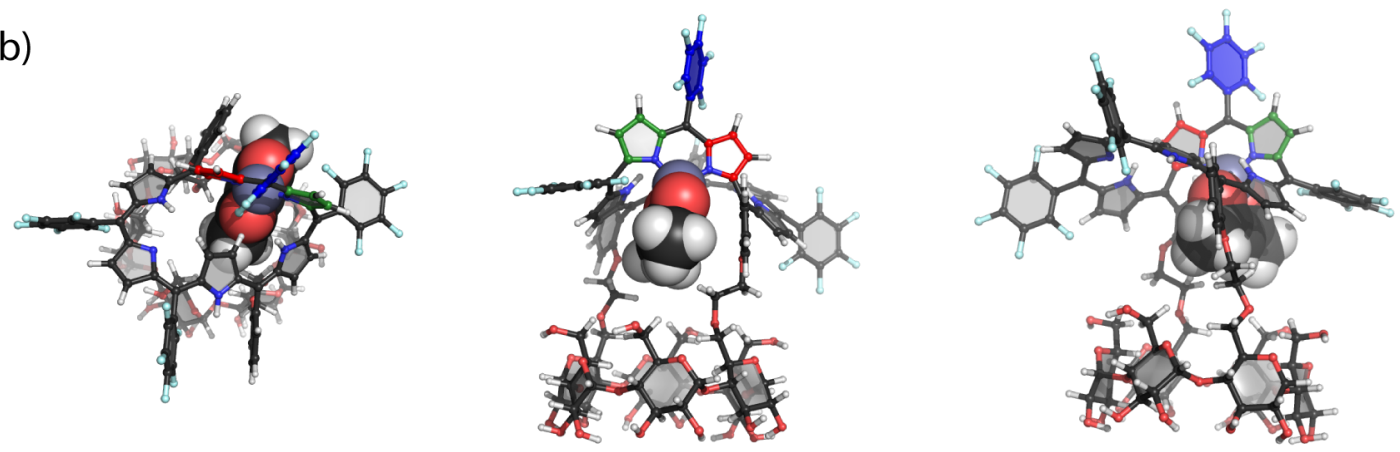

Geometry optimized (Avogadro, UFF force field, benzyl groups omitted for modelling) models of $(M)$ ${ }^{\mathrm{M}}[\mathbf{2 8}] \mathbf{2} \cdot \mathrm{ZnL}^{2}$ (from left to right : top view, view from the coordination site and side view) with either (a) down (observed) or (b) up (putative) orientation of the twisted pyrrole (in red; corresponding $\mathrm{N}$ atom up and down respectively).

The down orientation of the twisted pyrrole (a) leads to an exo-complexation where $L^{2}$ methyl groups are located in a region lacking protons (only three NOE correlations involving the ' $i{ }^{\prime}$ ' methyl group are observed with the inner pyrrole and the adjacent outer one, see below for corresponding distances). Conversely, the up orientation of the twisted pyrrole (b) leads to an endo-complexation where the $\mathrm{L}^{2}$ methyl groups are surrounded by protons (pyrroles, phenylethylenic linkers, benzyl groups [omitted] and cyclodextrin protons). Thus, the exo-complexation de facto cannot provide any NOE correlations with those groups, while the endo-complexation would give numerous NOE correlations. 
7.2.3 Distances between protons involved in NOE correlations with the bound $L^{2}$ of $(M)$ ${ }^{M}$ [28]2-ZnL ${ }^{2}$ (exo-complexation) and the corresponding NOESY region of interest
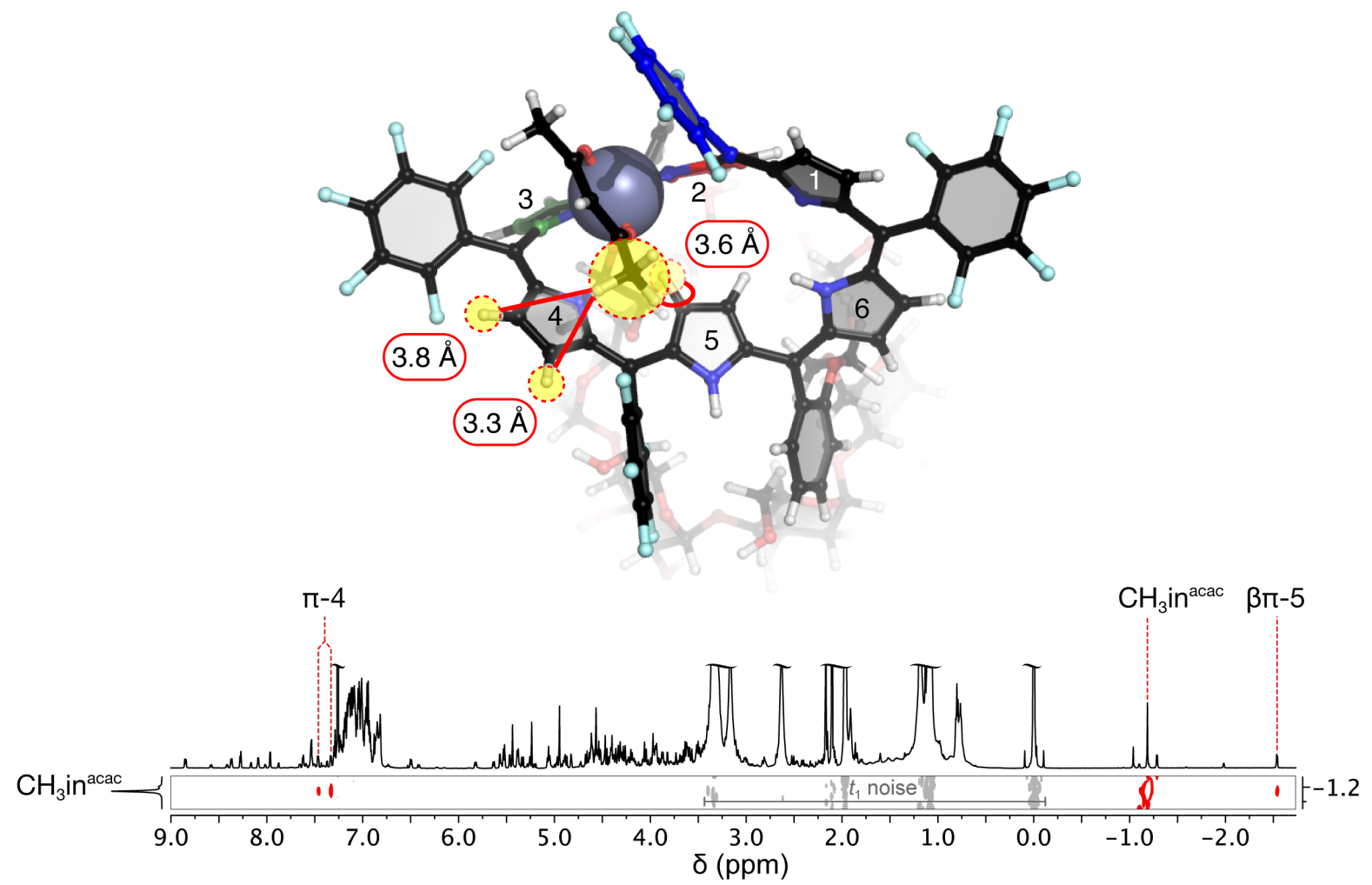

2D NOESY spectrum $\left(\mathrm{CDCl}_{3} / \mathrm{CD}_{3} \mathrm{OD} 9: 1,298 \mathrm{~K}, 600 \mathrm{MHz}\right)$. Correlation region concerning the $\mathrm{CH}_{3}$ in group of the acac ligand $\left(\mathrm{L}^{2}\right)$. 
7.2.4 ${ }^{1} \mathrm{H} \mathrm{NMR}\left(\mathrm{CDCl}_{3} / \mathrm{CD}_{3} \mathrm{OD} 9: 1,500 \mathrm{MHz}, 298 \mathrm{~K}\right)$ titration with $\mathrm{Zn}(\mathrm{OTf})_{2} / \mathrm{L}^{2}$ and subsequent evolution
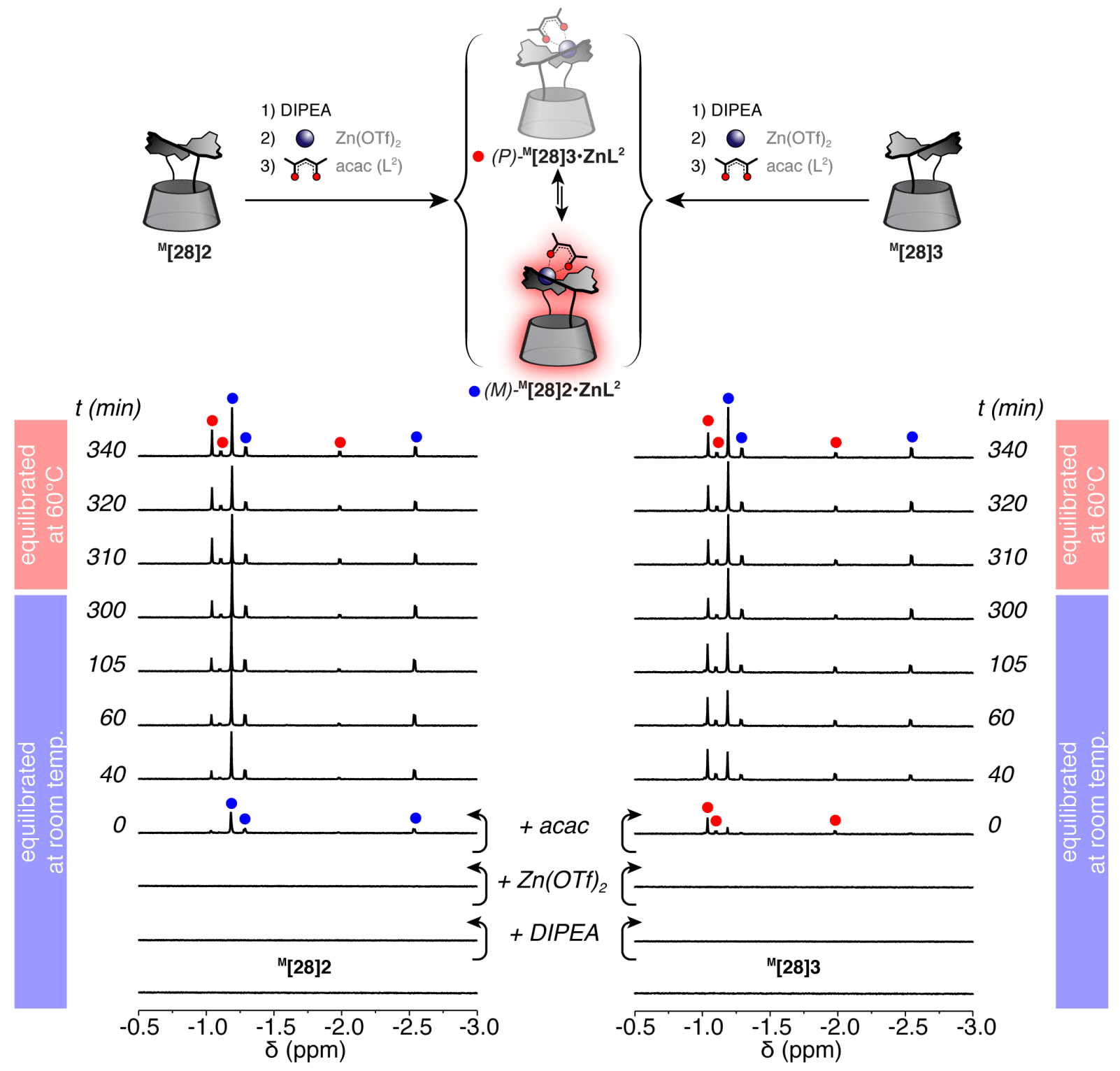


\subsubsection{D NMR and NMR description of (M)- ${ }^{M}[\mathbf{2 8}] 2 \cdot \mathrm{ZnL}^{2} /(P)-{ }^{M}[\mathbf{2 8}] 3 \cdot \mathrm{ZnL}^{\mathbf{2}}$ (75:25 d.r.)}

\subsubsection{NMR description}

Major complex: (M)- ${ }^{M}[\mathbf{2 8}] 2 \cdot \mathrm{ZnL}^{2}$

${ }^{1} \mathrm{H} \mathrm{NMR}\left(\mathrm{CDCl}_{3} / \mathrm{CD}_{3} \mathrm{OD} 9: 1,298 \mathrm{~K}, 600 \mathrm{MHz}\right)$ and ${ }^{13} \mathrm{C}$ from HSQC (600 $\mathrm{MHz}): \delta(\mathrm{ppm})$

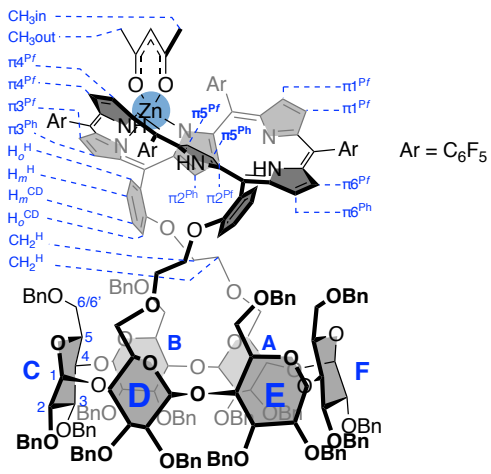

Hexaphyrin part

$(M)-{ }^{M}[28] 2 \cdot Z n L^{2}$

\begin{tabular}{|c|c|c|c|c|c|c|c|c|c|c|c|c|}
\hline$\beta-\pi$ & $\pi 1^{P f}$ & $\pi 1^{\text {Pf }}$ & $\pi 2^{\mathrm{Pf}}$ & $\pi 2^{\mathrm{Ph}}$ & $\pi 3^{\text {Ph }}$ & $\pi 3^{P f}$ & $\pi 4^{P f}$ & $\pi 4^{, P f}$ & $\pi 5^{\text {Pf }}$ & $\pi 5^{\mathrm{Ph}}$ & $\pi 6^{\mathrm{Ph}}$ & $\pi 6^{\mathrm{Pf}}$ \\
\hline $\mathrm{H}$ & 7.63 & 7.16 & 4.96 & 4.96 & 7.54 & 7.97 & 7.34 & 7.47 & -1.29 & -2.54 & 8.28 & 8.09 \\
\hline C & 131.0 & 131.3 & $\begin{array}{c}120.2 \text { or } \\
125.5\end{array}$ & $\begin{array}{c}125.5 \text { or } \\
120.2\end{array}$ & 122.7 & 129.5 & 123.3 & 122.6 & 117.1 & 119.9 & 128.6 & 124.9 \\
\hline
\end{tabular}

\section{Cyclodextrin part}

\begin{tabular}{|c|c|c|c|c|c|c|c|}
\hline $\begin{array}{c}\text { CD Unit } \\
\text { (Bn) }\end{array}$ & $\begin{array}{c}\text { CD Unit } \\
\text { (linker) }\end{array}$ & $\begin{array}{c}\mathrm{A} \\
\text { (linker) }\end{array}$ & $\begin{array}{c}B \\
(B n-i n)\end{array}$ & $\begin{array}{c}\mathrm{C} \\
\text { (Bn-out) }\end{array}$ & $\begin{array}{c}\mathrm{D} \\
\text { (linker) }\end{array}$ & $\begin{array}{c}E \\
(B n-i n)\end{array}$ & $\begin{array}{c}F \\
\text { (Bn-out) }\end{array}$ \\
\hline $\mathrm{H}-1$ & $\mathrm{H}-1$ & 3.74 & 4.84 & 5.58 & 3.83 & 4.63 & 5.48 \\
\hline C-1 & C-1 & 97.4 & 97.0 & 96.5 & 96.9 & 96.7 & 96.6 \\
\hline $\mathrm{H}-2$ & $\mathrm{H}-2$ & 2.60 & 3.38 & 3.38 & 2.68 & 3.22 & 3.31 \\
\hline C-2 & C-2 & 79.8 & 77.7 or 78.8 & 77.7 or 78.8 & 79.8 & 78.67 & 77.6 \\
\hline $\mathrm{H}-3$ & $\mathrm{H}-3$ & 3.64 & 3.68 & 3.96 & 3.66 & 3.61 & 4.06 \\
\hline C-3 & $C-3$ & 81.6 & 80.6 & 81.0 & 81.7 & 81.4 & 79.8 \\
\hline $\mathrm{H}-4$ & $\mathrm{H}-4$ & 3.28 & 3.63 & 4.21 & 3.46 & 3.51 & 3.89 \\
\hline C-4 & $C-4$ & 70.8 & 80.2 & 79.9 & 70.8 & 79.9 & 81.0 \\
\hline $\mathrm{H}-5$ & $\mathrm{H}-5$ & 3.12 & 3.30 & 3.5 & 3.29 & 3.17 & 3.31 \\
\hline C-5 & $C-5$ & 70.2 & 70.9 & 71.2 & 70.8 & 71.0 & 71.0 \\
\hline $\mathrm{H}-6$ & $\mathrm{H}-6$ & 1.85 & 2.82 & 3.60 & 2.08 & 2.41 & 3.41 \\
\hline $\mathrm{H}-6^{\prime}$ & H-6' & 3.10 & 3.18 & 4.18 & 3.30 & 3.12 & 3.96 \\
\hline C-6 & C-6 & 69.2 & 65.9 & 70.2 & 68.2 & 66.8 & 69.8 \\
\hline $\mathrm{CH} \mathrm{H}^{\mathrm{Bn}-6}$ & $\mathrm{CH} \mathrm{H}^{\mathrm{CD}}$ & 2.62 & 3.41 & nd & 3.31 & 2.51 & $n d$ \\
\hline $\mathrm{CH} H^{\mathrm{Bn}-6}$ & $\mathrm{CH} H^{\mathrm{CD}}$ & 3.01 & 4.30 & nd & 3.46 & 3.58 & $n d$ \\
\hline $\mathrm{CH}_{2}^{\mathrm{Bn}-6}$ & $\mathrm{CH}_{2}{ }^{\mathrm{CD}}$ & 69.0 & 72.7 & nd & 70.9 & 72.5 & $n d$ \\
\hline- & $\mathrm{CH} \mathrm{H}^{\mathrm{H}}$ & 1.80 & - & - & 1.85 & & - \\
\hline- & $\mathrm{CH} H^{\mathrm{H}}$ & 1.14 & - & - & 2.47 & - & - \\
\hline- & $\mathrm{CH}_{2}{ }^{\mathrm{H}}$ & 70.3 & - & - & 70.2 & - & - \\
\hline $\mathrm{H}_{o}{ }^{\mathrm{Bn}-6}$ & $\mathrm{H}_{0} \mathrm{CD}$ & 6.50 & 5.38 & nd & 7.15 & 5.53 & $n d$ \\
\hline $\mathrm{C}_{0}{ }^{\mathrm{Bn}-6}$ & $\mathrm{C}_{0}{ }^{\mathrm{CD}}$ & 114.3 & 127.1 & nd & 118.0 & 127.1 & $n d$ \\
\hline $\mathrm{H}_{m}{ }^{\mathrm{Bn}-6}$ & $\mathrm{H}_{m}{ }^{\mathrm{CD}}$ & 7.23 & 3.94 & nd & 7.54 & 5.06 & $n d$ \\
\hline $\mathrm{C}_{m}^{\mathrm{Bn}-6}$ & $\mathrm{C}_{m}{ }^{\mathrm{CD}}$ & 130.7 & 125.5 & nd & 129.2 & 126.8 & $n d$ \\
\hline $\mathrm{H}_{p}{ }^{\mathrm{Bn}-6}$ & $\mathrm{H}_{m}{ }^{\mathrm{H}}$ & 7.24 & 2.81 & nd & 7.53 & 4.90 & $n d$ \\
\hline $\mathrm{C}_{p}{ }^{\mathrm{Bn}-6}$ & $\mathrm{C}_{m}{ }^{\mathrm{H}}$ & 120.8 & 125.3 & nd & 122.8 & 126.3 & $n d$ \\
\hline- & $\mathrm{H}_{0}{ }^{\mathrm{H}}$ & 8.86 & - & - & 8.38 & - & - \\
\hline - & $\mathrm{C}_{0}{ }^{\mathrm{H}}$ & 136.1 & - & - & 135.6 & - & - \\
\hline
\end{tabular}

nd : not determined

\section{Ligand part}

\begin{tabular}{|c|c|}
\hline ligand & acac \\
\hline $\mathrm{CH}_{3}$-in & -1.19 \\
$\mathrm{CH}_{3}$-in & 23.8 \\
$\mathrm{CH}_{3}$-out & 1.13 \\
$\mathrm{CH}_{3}$-out & 26.2 \\
\hline
\end{tabular}

${ }^{19} \mathrm{~F} \mathrm{NMR}\left(\mathrm{CDCl}_{3} / \mathrm{CD}_{3} \mathrm{OD} 9: 1,298 \mathrm{~K}, 565 \mathrm{MHz}\right): \delta-135.99$ (d, $\left.1 \mathrm{~F}, J=24.6 \mathrm{~Hz}, \mathrm{~F}_{\text {ortho }}\right),-136.71$ (d, $1 \mathrm{~F}, J=24.3$ $\left.\mathrm{Hz}, \mathrm{F}_{\text {ortho }}\right),-137.05$ (dt, $\left.1 \mathrm{~F}, J=8.4,24.5 \mathrm{~Hz}, \mathrm{~F}_{\text {ortho }}\right),-137.17$ (d, $1 \mathrm{~F}, J=21.5 \mathrm{~Hz}, \mathrm{~F}_{\text {ortho }}$ ), -137.33 (dt, $1 \mathrm{~F}, J=$ 7.8, 25.4 Hz, $\left.F_{\text {ortho }}\right),-138.31\left(\mathrm{~d}, 1 \mathrm{~F}, J=24.8 \mathrm{~Hz}, \mathrm{~F}_{\text {ortho }}\right),-138.52\left(\mathrm{~d}, 1 \mathrm{~F}, J=23.8 \mathrm{~Hz}, \mathrm{~F}_{\text {ortho }}\right),-141.66$ (d, $1 \mathrm{~F}, J$ $\left.=21.8 \mathrm{~Hz}, \mathrm{~F}_{\text {ortho }}\right),-151.81\left(\mathrm{t}, 1 \mathrm{~F}, J=21.3 \mathrm{~Hz}, \mathrm{~F}_{\text {para }}\right),-153.07\left(\mathrm{t}, 1 \mathrm{~F}, J=21.1 \mathrm{~Hz}, \mathrm{~F}_{\text {para }}\right),-154.07(\mathrm{t}, 1 \mathrm{~F}, J=$ $\left.21.2 \mathrm{~Hz}, \mathrm{~F}_{\text {para }}\right),-154.59\left(\mathrm{t}, 1 \mathrm{~F}, \mathrm{~J}=20.2 \mathrm{~Hz}, \mathrm{~F}_{\text {para }}\right),-159.79\left(\mathrm{t}_{\mathrm{b}}, 1 \mathrm{~F}, J=24.3 \mathrm{~Hz}, \mathrm{~F}_{\text {meta }}\right),-160.78\left(\mathrm{t}_{\mathrm{b}}, 1 \mathrm{~F}, J=22.6\right.$ $\left.\mathrm{Hz}, \mathrm{F}_{\text {meta }}\right),-161.80\left(\mathrm{t}_{\mathrm{b}}, 1 \mathrm{~F}, J=21.7 \mathrm{~Hz}, \mathrm{~F}_{\text {meta }}\right),-162.14\left(\mathrm{~m}, 3 \mathrm{~F}, \mathrm{~F}_{\text {meta }}\right),-163.02\left(\mathrm{t}_{\mathrm{b}}, 1 \mathrm{~F}, \mathrm{~J}=22.5 \mathrm{~Hz}, \mathrm{~F}_{\text {meta }}\right)$, $163.27\left(\mathrm{t}_{\mathrm{b}}, 1 \mathrm{~F}, J=23.8 \mathrm{~Hz}, \mathrm{~F}_{\text {meta }}\right)$. 
${ }^{1} \mathrm{H}$ NMR $\left(\mathrm{CDCl}_{3} / \mathrm{CD}_{3} \mathrm{OD} 9: 1,298 \mathrm{~K}, 600 \mathrm{MHz}\right)$ and ${ }^{13} \mathrm{C}$ from HSQC (600 MHz):

Due to weak intensities and strong overlapping of the signals belonging to the cyclodextrin, only selected signals belonging to the Möbius hexaphyrin ring and the phenyl linkers are reported in section 7.2.9 (vide infra).

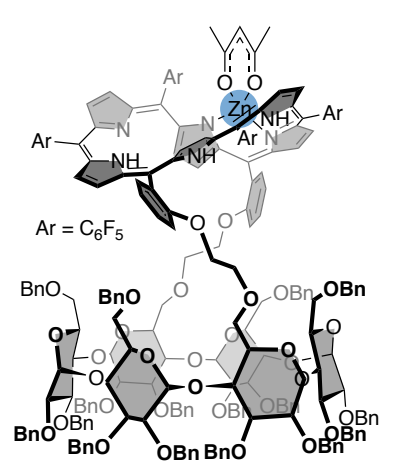

$(P)-{ }^{M}[28] 3 \cdot \mathrm{ZnL}^{2}$

${ }^{19} \mathrm{~F}$ NMR $\left(\mathrm{CDCl}_{3} / \mathrm{CD}_{3} \mathrm{OD} 9: 1,298 \mathrm{~K}, 565 \mathrm{MHz}\right.$, partial description due weak intensity and strong overlapping): $\delta-136.13\left(\mathrm{~d}_{\mathrm{b}}, 1 \mathrm{~F}, J=21.7 \mathrm{~Hz}, \mathrm{~F}_{\text {ortho }}\right),-136.99\left(\mathrm{~m}, 3 \mathrm{~F}, \mathrm{~F}_{\text {ortho }}\right),-137.58\left(\mathrm{~d}_{\mathrm{b}}, 1 \mathrm{~F}, J=24.4 \mathrm{~Hz}\right.$, $\left.F_{\text {ortho }}\right),-138.12\left(d_{b}, 1 F, J=23.0 \mathrm{~Hz}, F_{\text {ortho }}\right),-138.37\left(d_{b}, 1 F, J=20.6 \mathrm{~Hz}, F_{\text {ortho }}\right),-141.53\left(d_{b}, 1 F, J=22.1 \mathrm{~Hz}\right.$, $\left.F_{\text {ortho }}\right),-152.31\left(\mathrm{t}_{\mathrm{b}}, 1 \mathrm{~F}, J=20.5 \mathrm{~Hz}, \mathrm{~F}_{\text {para }}\right),-153.18\left(\mathrm{t}_{\mathrm{b}}, 1 \mathrm{~F}, J=20.5 \mathrm{~Hz}, \mathrm{~F}_{\text {para }}\right),-152.31\left(\mathrm{t}_{\mathrm{b}}, 1 \mathrm{~F}, J=21.1 \mathrm{~Hz}\right.$, $\left.F_{\text {meta }}\right),-153.18\left(t_{b}, 1 F, J=20.8 \mathrm{~Hz}, F_{\text {meta }}\right),-160.78\left(m, 1 F, F_{\text {meta }}\right),-161.16\left(t_{b}, 1 F, J=21.9 \mathrm{~Hz}, F_{\text {meta }}\right),-161.99$ $\left(m, 1 F, F_{\text {meta }}\right),-163.17\left(m, 1 F, F_{\text {meta }}\right)$. 


\subsubsection{2 $2 \mathrm{D} \mathrm{COSY}\left(\mathrm{CDCl}_{3} / \mathrm{CD}_{3} \mathrm{OD} 9: 1,298 \mathrm{~K}, 600 \mathrm{MHz}\right)$}

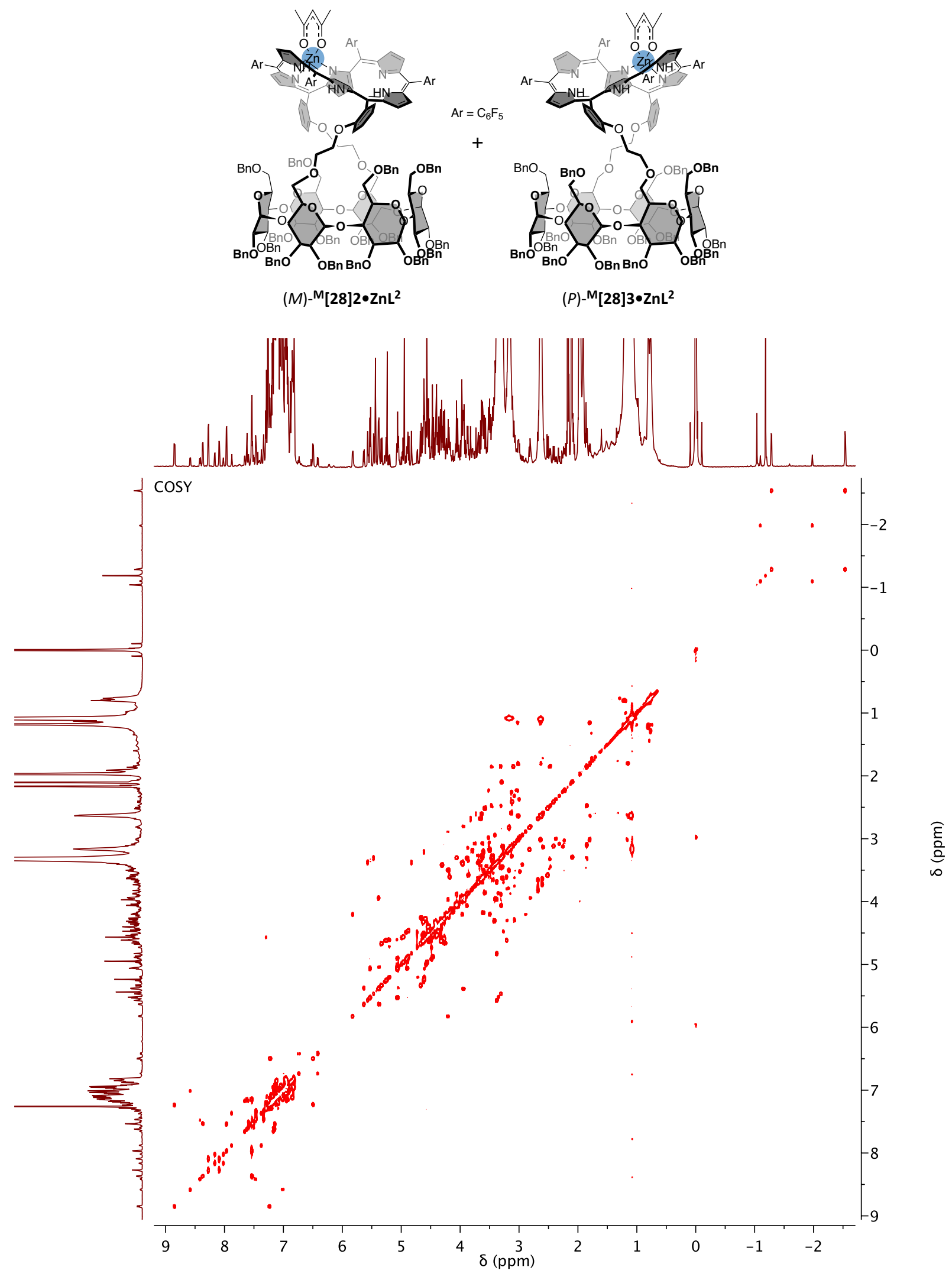




\subsubsection{2D TOCSY $\left(\mathrm{CDCl}_{3} / \mathrm{CD}_{3} \mathrm{OD}\right.$ 9:1, $\left.298 \mathrm{~K}, 600 \mathrm{MHz}\right)$}
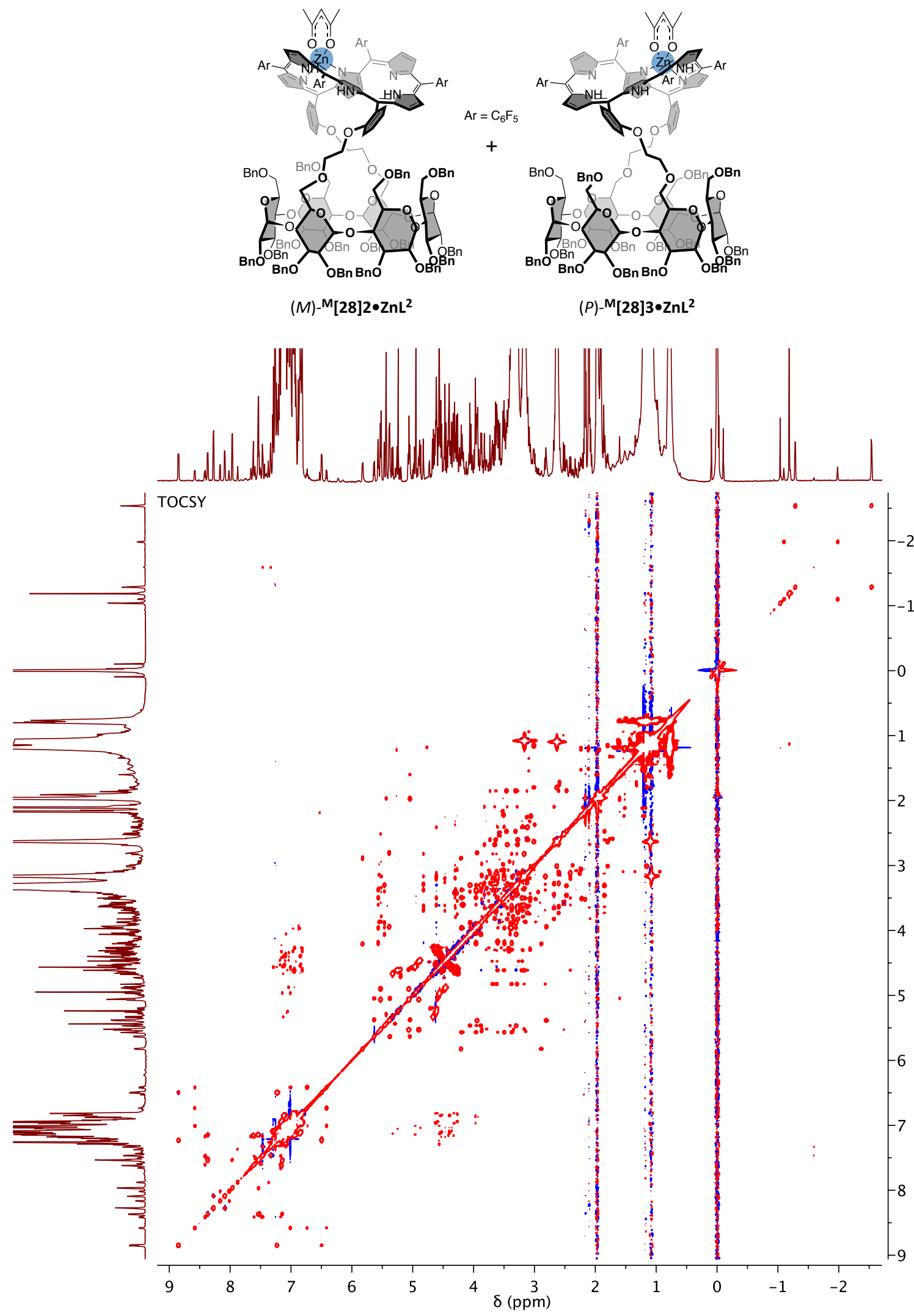

$\hat{\varepsilon}$
مo
0 
7.2.5.4 2D HSQC $\left(\mathrm{CDCl}_{3} / \mathrm{CD}_{3} \mathrm{OD} 9: 1,298 \mathrm{~K}, 600 \mathrm{MHz}\right)$
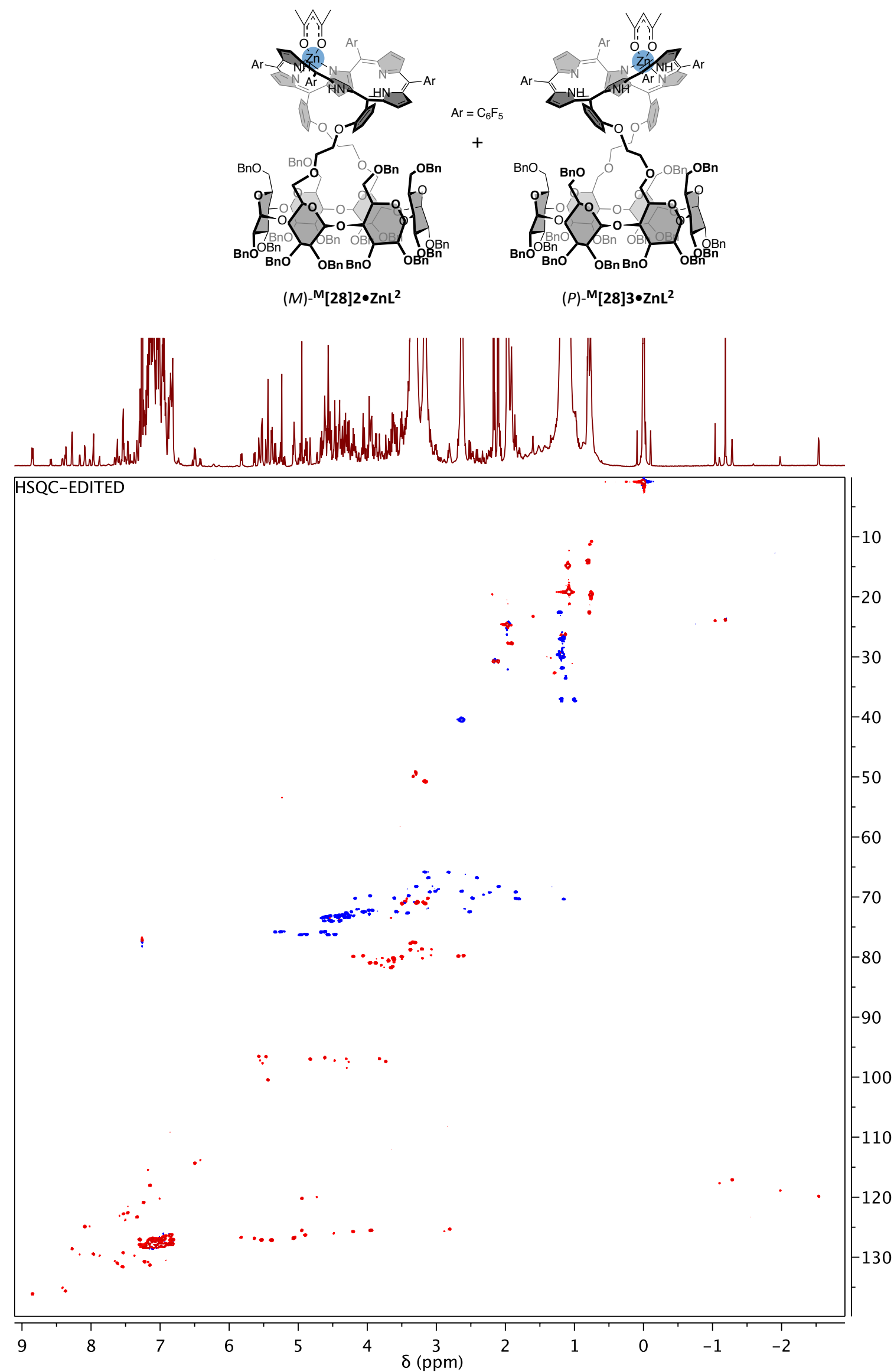


\subsubsection{2D NOESY $\left(\mathrm{CDCl}_{3} / \mathrm{CD}_{3} \mathrm{OD}\right.$ 9:1, $\left.298 \mathrm{~K}, 600 \mathrm{MHz}\right)$}
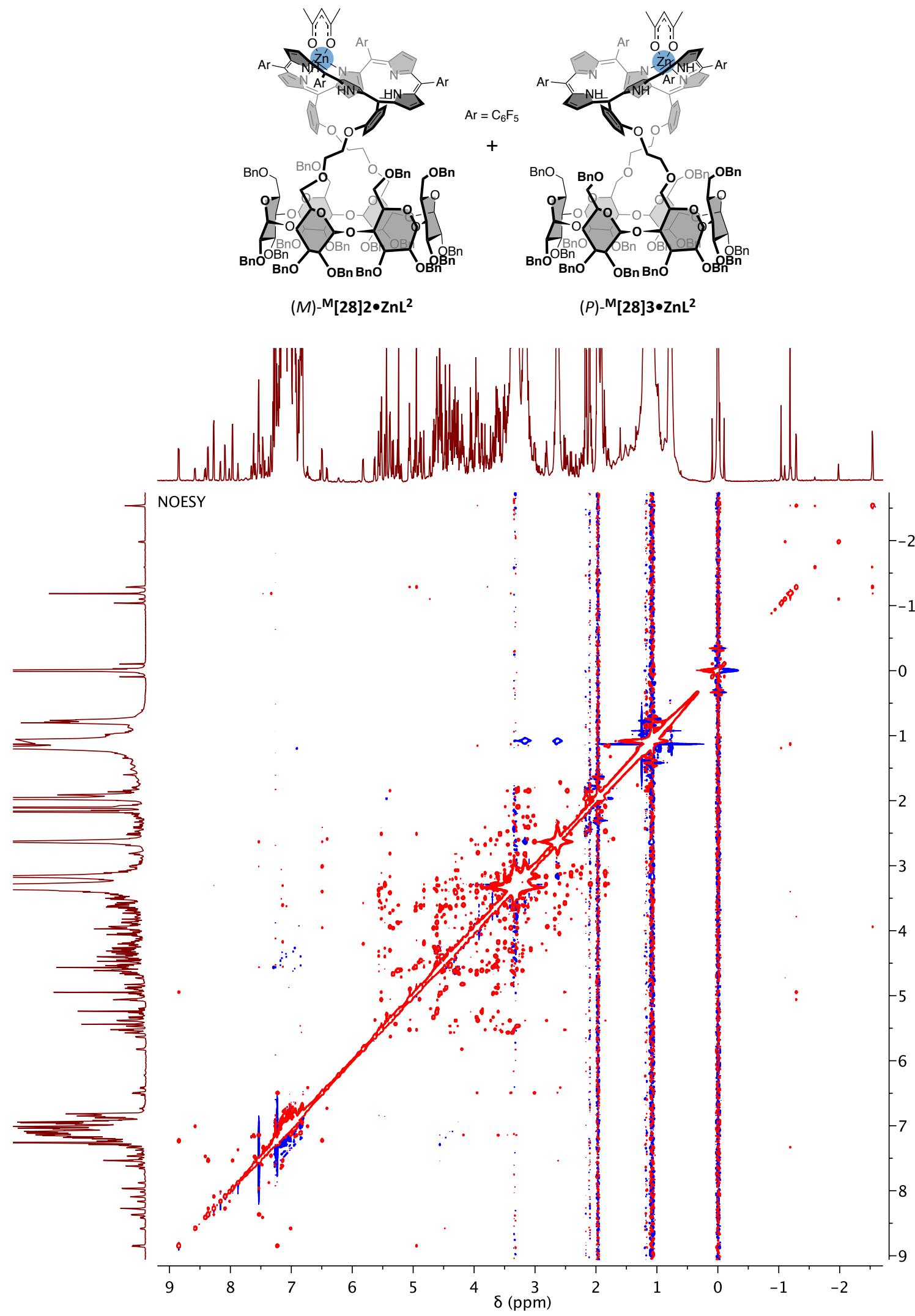

$\hat{\varepsilon}$
مo
0 
7.2.5.6 2D ${ }^{19} \mathrm{~F} \mathrm{COSY}\left(\mathrm{CDCl}_{3} / \mathrm{CD}_{3} \mathrm{OD}\right.$ 9:1, $\left.298 \mathrm{~K}, 565 \mathrm{MHz}\right)$

Labelling of ortho/ortho' fluorine atoms and corresponding $\Delta \delta$ values shown for the major complex only.

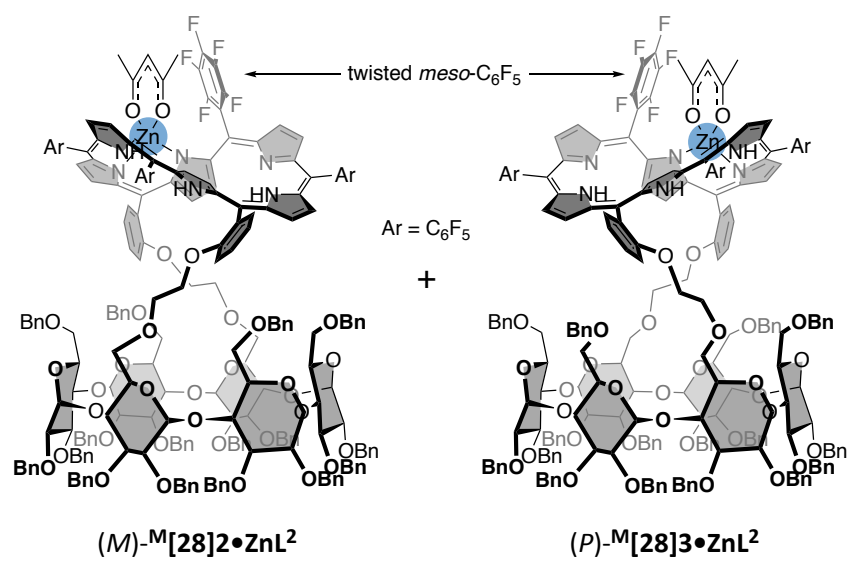

peripheral meso positions

twisted meso

( $x$ 3)

position
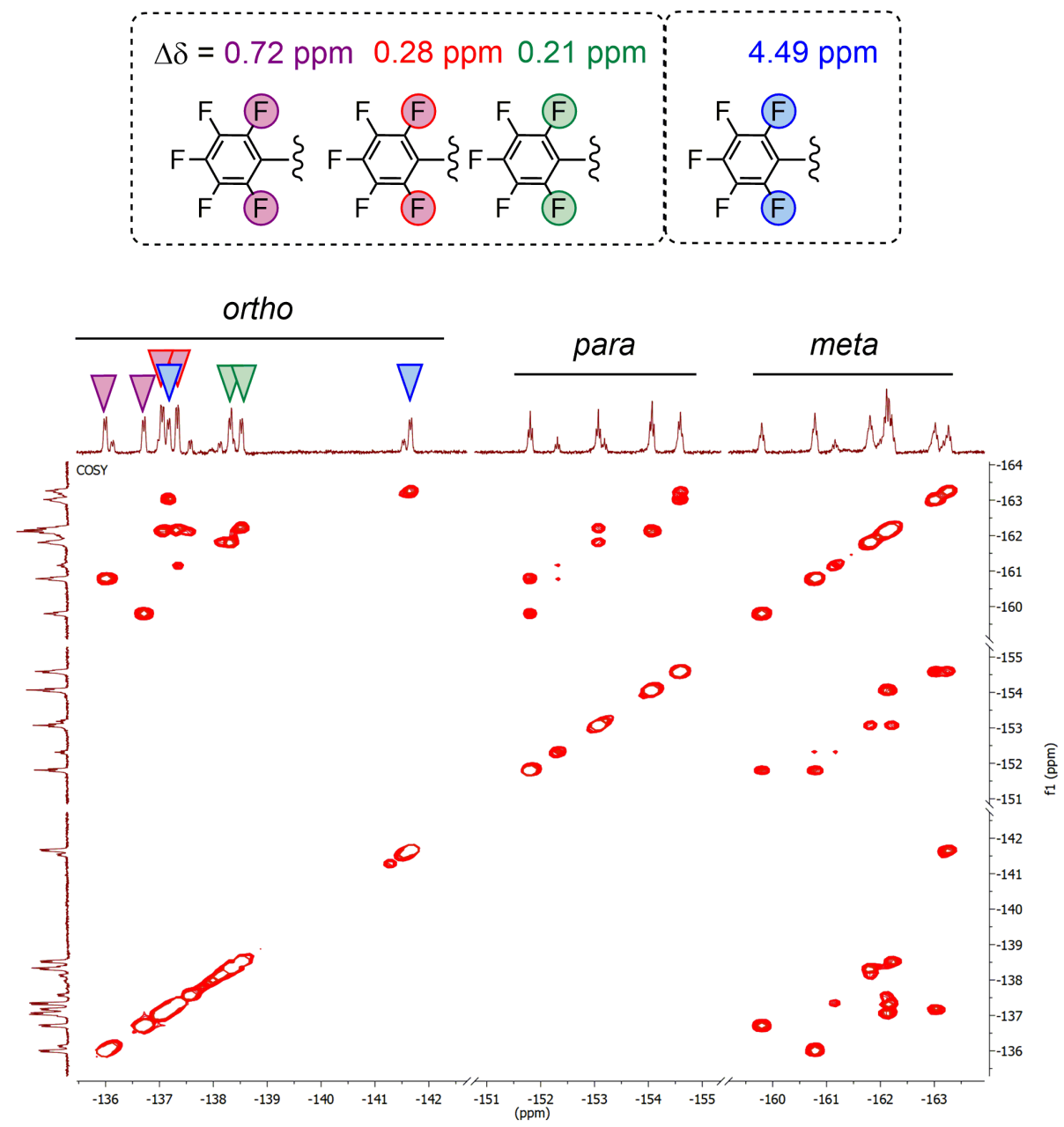
7.2.6 ${ }^{1} H$ NMR spectra of (M)- ${ }^{M}[28] 2 \cdot Z n L^{n} /(P)-{ }^{M}[28] 3 \cdot Z n L^{n}\left(C D C l_{3} / C D_{3} O D\right.$ 9:1, $298 K, 376$ $\mathrm{MHz}$

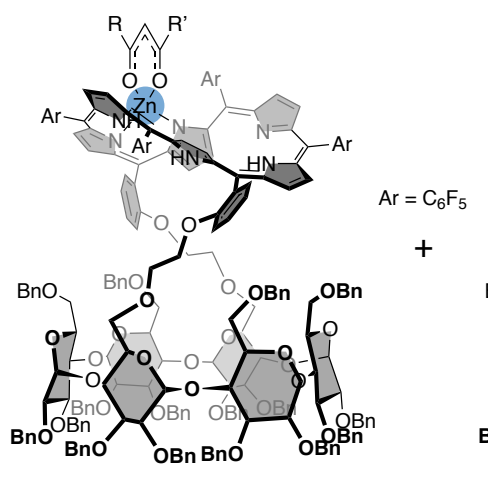

$(M){ }^{M}[28] 2 \bullet Z n L^{n}$

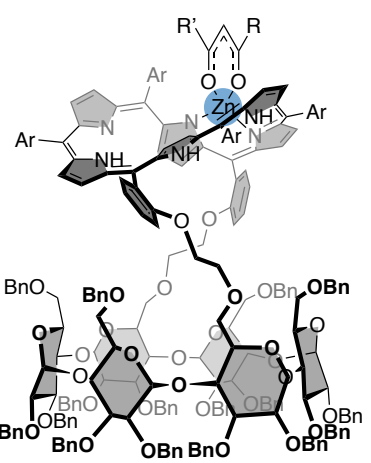

$(P)-{ }^{M}[28] 3 \cdot Z n L^{n}$
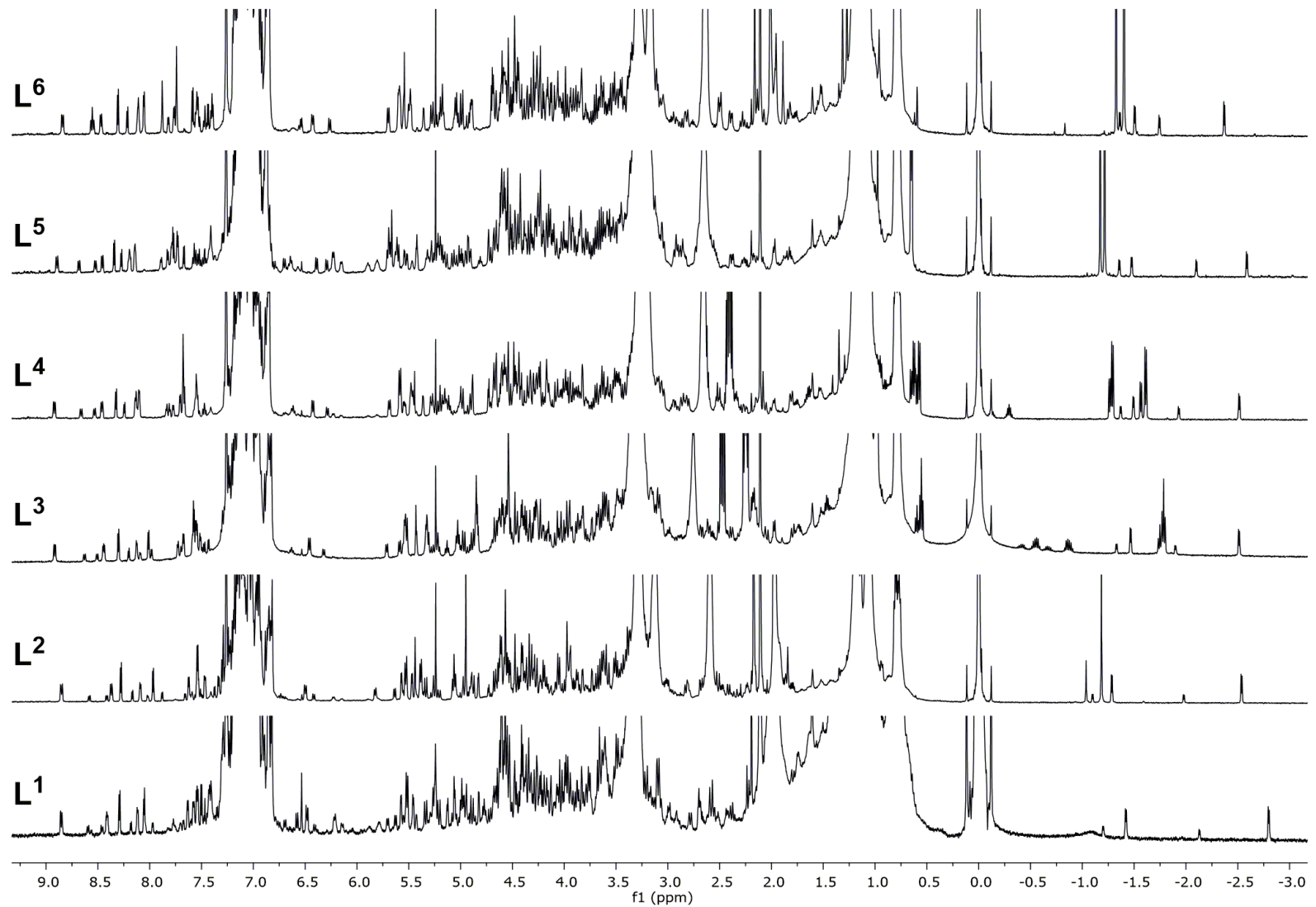
7.2.7 ${ }^{19} \mathrm{~F} N M R$ spectra of $(M)-{ }^{M}[\mathbf{2 8}] 2 \cdot \mathrm{ZnL}^{n} /(P)-{ }^{M}[\mathbf{2 8}] 3 \cdot \mathrm{ZnL}^{n}\left(\mathrm{CDCl}_{3} / \mathrm{CD}_{3} O D\right.$ 9:1, $298 \mathrm{~K}, 500$ $\mathrm{MHz})$

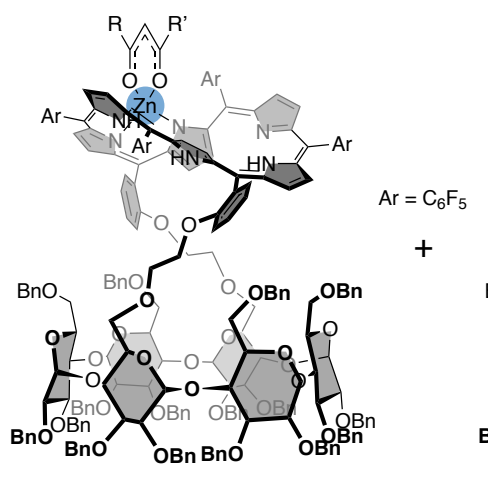

$(M){ }^{M}[28] 2 \bullet Z n L^{n}$

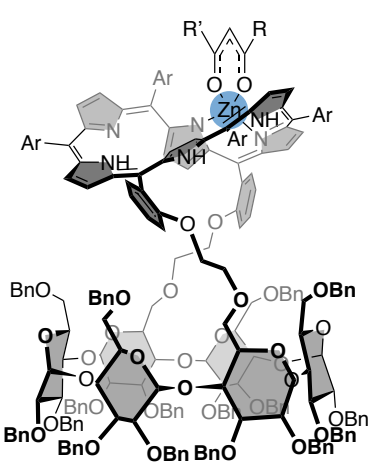

$(P)-{ }^{\mathrm{M}}[28] 3 \cdot \mathrm{ZnL}{ }^{\mathrm{n}}$
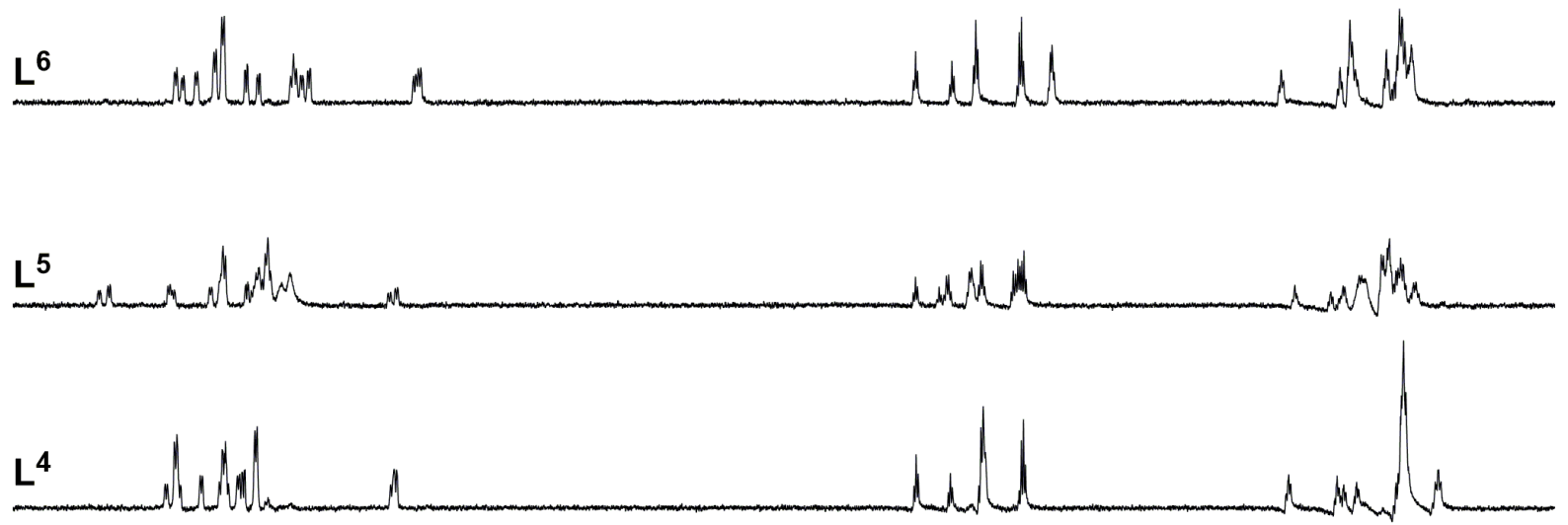

$\mathbf{L}^{3}$ hun

hunch An

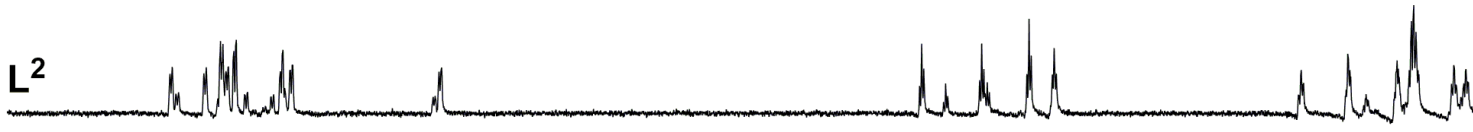

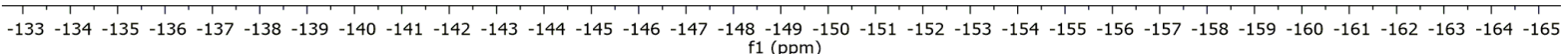


7.2.8 Normalized UV-vis absorption spectra of (M)- ${ }^{M}[28] 2 \cdot \mathrm{ZnL}^{n} /(P)-{ }^{M}[\mathbf{2 8}] 3 \cdot \mathrm{Zn} L^{n}$ $\left(\mathrm{CHCl}_{3} / \mathrm{CH}_{3} \mathrm{OH}\right.$ 9:1)
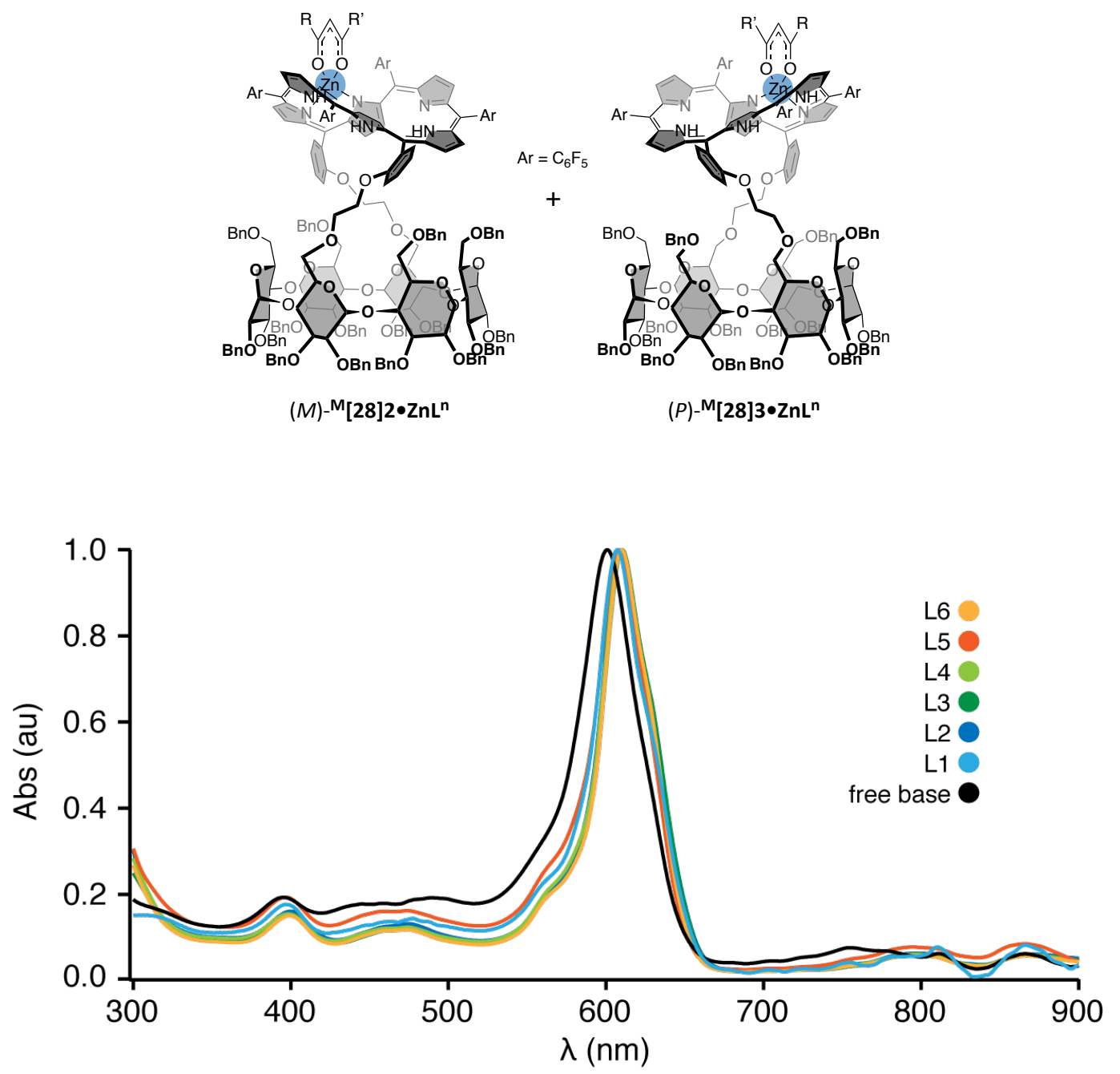

Normalized UV-vis absorption spectra of ${ }^{\mathrm{M}}[\mathbf{2 8}] 2 /{ }^{\mathrm{M}}[\mathbf{2 8}] 3$ and of the complexes $(M){ }^{\mathrm{M}}[\mathbf{2 8}] 2 \cdot \mathrm{ZnL}^{1-5} /(P)$ ${ }^{\mathrm{M}}[\mathbf{2 8}] 3 \cdot \mathrm{ZnL}^{1-5}\left(\mathrm{CHCl}_{3} / \mathrm{CH}_{3} \mathrm{OH}\right.$ 9:1). 
7.2.9 Selected ${ }^{1} H$ NMR chemical shifts (in ppm) of (M)- ${ }^{M}[\mathbf{2 8}] 2 \cdot \mathrm{ZnL}^{n} /(P){ }^{M}[\mathbf{2 8}] 3 \cdot \mathrm{Zn} L^{n}$ $\left(\mathrm{CDCl}_{3} / \mathrm{CD}_{3} \mathrm{OD}\right.$ 9:1, $\left.298 \mathrm{~K}\right)$

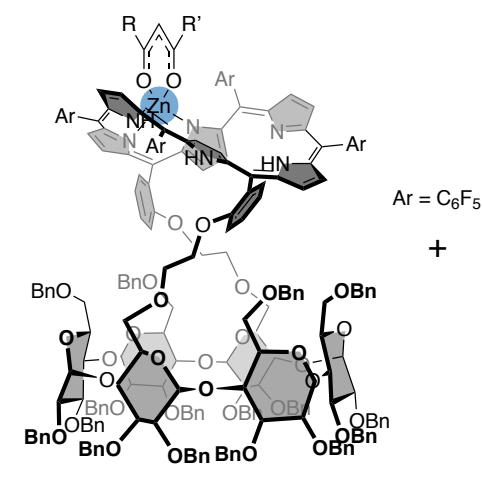

$(M){ }^{M}[28] 2 \bullet Z n L^{n}$

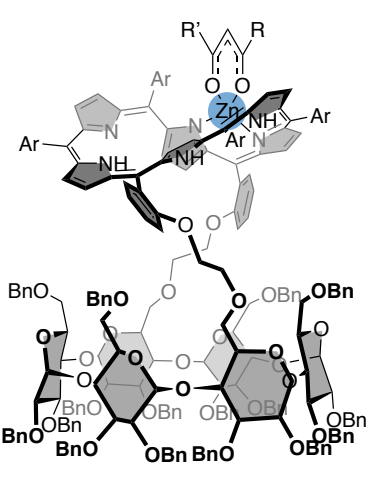

$(P)-{ }^{\mathrm{M}}[28] 3 \cdot \mathrm{Zn} L^{\mathrm{n}}$

\begin{tabular}{lccc}
\hline & $\beta \pi$ out & $\beta \pi$ twisted & $\beta \pi$ in \\
\hline$L^{1}(M)$ & $8.30 / 8.12,8.06 / 7.51,7.64 / 7.19,7.60 / 7.46$ & $5.15,5.08$ & $-1.42,-2.80$ \\
$L^{1}(P)$ & $8.19 / 8.05,7.98 / 7.35,7.73 / 7.63,7.70 / 7.25$ & $n d$ & $-1.20,-2.13$ \\
$L^{2}(M)$ & $8.28 / 8.09,7.97 / 7.54,7.63 / 7.16,7.47 / 7.34$ & $4.94,4.94$ & $-1.29,-2.54$ \\
$L^{2}(P)$ & $8.16 / 8.02,7.88 / 7.37,7.66 / 7.17,7.61 / 7.47$ & $4.73,4.48$ & $-1.10,-1.98$ \\
$L^{3}(M)$ & $8.30 / 8.12,8.01 / 7.57,7.67 / 7.20,7.58 / 7.51$ & $4.83,4.85$ & $-1.47,-2.51$ \\
$L^{3}(P)$ & $8.20 / 8.09,7.98 / 7.43,7.73 / 7.23,7.72 / 7.69$ & $4.55,4.28$ & $-1.33,-1.90$ \\
$L^{4}(M)$ & $8.32 / 8.13,8.09 / 7.67,7.70 / 7.24,7.68 / 7.68$ & $4.66,4.73$ & $-1.49,-2.51$ \\
$L^{4}(P)$ & $8.25 / 8.12,8.10 / 7.55,7.84 / 7.81,7.77 / 7.28$ & $4.33,4.17$ & $-1.37,-1.93$ \\
$L^{5}(M)$ & $n d$ & $n d$ & $-1.48,-2.59$ \\
$L^{5}(P)$ & $n d$ & $n d$ & $-1.36,-2.10$ \\
$L^{6} L(M)$ & $8.30 / 8.11,8.06 / 7.58,7.76 / 7.39,7.74(\times 2)$ & $4.48,4.70$ & $-1.51,-2.37$ \\
$L^{6} L(P)$ & $8.21 / 8.10,8.05 / 7.44,7.88(\times 2), 7.82 / 7.40$ & $4.18,4.12$ & $-1.36,-1.74$ \\
$L^{6} S(M)$ & $n d$ & $n d$ & $-1.29,-2.66$ \\
$L^{6} S(P)$ & $n d$ & $n d$ & $-1.19,-2.16$ \\
\hline
\end{tabular}

\begin{tabular}{|c|c|c|}
\hline & HArmeso (adjacent to $\beta \pi$ twisted) & HArmeso (adjacent to $\beta \pi$ in) \\
\hline $\mathrm{L}^{1}(M)$ & $8.85,7.27,7.25,6.49$ & $8.41,7.54(\times 2), 7.15$ \\
\hline $\mathrm{L}^{1}(P)$ & $8.60,7.04,6.77,6.42$ & $8.46,7.56,7.48,7.16$ \\
\hline$L^{2}(M)$ & $8.86,7.24,7.23,6.50$ & $8.38,7.54,7.53,7.15$ \\
\hline $\mathrm{L}^{2}(P)$ & $8.58,7.01,6.74,6.42$ & $8.41,7.59,7.50,7.17$ \\
\hline $\mathrm{L}^{3}(M)$ & $8.91,7.22(x 2), 6.45$ & $8.44,7.55(\times 2), 7.13$ \\
\hline $\mathrm{L}^{3}(P)$ & $8.62,6.97,6.63,6.32$ & $8.50,7.48(\times 2), 7.11$ \\
\hline$L^{4}(M)$ & $8.92,7.21(x 2), 6.42$ & $8.46,7.55(\times 2), 7.10$ \\
\hline $\mathrm{L}^{4}(P)$ & $8.66,6.95,6.61,6.28$ & $8.53,7.48,7.55,7.09$ \\
\hline $\mathrm{L}^{5}(M)$ & nd & nd \\
\hline $\mathrm{L}^{5}(P)$ & nd & nd \\
\hline$L^{6} L(M)$ & $8.84,7.18(x 2), 6.42$ & $8.47,7.54(\times 2), 7.08$ \\
\hline$L^{6} L(P)$ & $8.56,6.91,6.53,6.28$ & $8.54,7.46,7.53,7.08$ \\
\hline$L^{6} S(M)$ & nd & nd \\
\hline$L^{6} S(P)$ & nd & nd \\
\hline
\end{tabular}

